\title{
Unravelling von Willebrand Factor
}

Citation for published version (APA):

van der Vorm, L. N. (2020). Unravelling von Willebrand Factor: Active von Willebrand Factor in conditions of (un)balanced haemostasis. [Doctoral Thesis, Maastricht University]. Optima Grafische Communicatie. https://doi.org/10.26481/dis.20200710lv

Document status and date:

Published: 01/01/2020

DOI:

10.26481/dis.20200710lv

Document Version:

Publisher's PDF, also known as Version of record

\section{Please check the document version of this publication:}

- A submitted manuscript is the version of the article upon submission and before peer-review. There can be important differences between the submitted version and the official published version of record.

People interested in the research are advised to contact the author for the final version of the publication, or visit the DOI to the publisher's website.

- The final author version and the galley proof are versions of the publication after peer review.

- The final published version features the final layout of the paper including the volume, issue and page numbers.

Link to publication

\footnotetext{
General rights rights.

- You may freely distribute the URL identifying the publication in the public portal. please follow below link for the End User Agreement:

www.umlib.nl/taverne-license

Take down policy

If you believe that this document breaches copyright please contact us at:

repository@maastrichtuniversity.nl

providing details and we will investigate your claim.
}

Copyright and moral rights for the publications made accessible in the public portal are retained by the authors and/or other copyright owners and it is a condition of accessing publications that users recognise and abide by the legal requirements associated with these

- Users may download and print one copy of any publication from the public portal for the purpose of private study or research.

- You may not further distribute the material or use it for any profit-making activity or commercial gain

If the publication is distributed under the terms of Article $25 \mathrm{fa}$ of the Dutch Copyright Act, indicated by the "Taverne" license above, 


\section{UNRAVELLING VON WILLEBRAND FACTOR Active von Willebrand Factor in conditions of (un)balanced haemostasis}

Lisa Nynke van der Vorm 
The research in this thesis was supported by an unrestricted grant from Synapse Research Institute. The design and printing of this thesis were supported by Synapse Research Institute.

(C) Lisa Nynke van der Vorm (2020)

No part of this book may be reproduced or transmitted to any form or by any means without prior permission in writing by the author, or when appropriate, by the publishers of the publication.

ISBN: 978-94-6361-436-8

Layout and printed by: Optima Grafische Communicatie, Rotterdam, the Netherlands (www.ogc.nl) 


\title{
UNRAVELLING VON WILLEBRAND FACTOR Active von Willebrand Factor in conditions of (un)balanced haemostasis
}

\author{
PROEFSCHRIFT \\ Ter verkrijging van de graad van doctor aan de Universiteit Maastricht, \\ op gezag van de Rector Magnificus, prof. dr. Rianne M. Letschert \\ volgens het besluit van het College van Decanen, \\ in het openbaar te verdedigen op \\ 10 juli 2020 om 10.00 uur \\ door \\ Lisa Nynke van der Vorm \\ Geboren op 25 oktober 1993 \\ te Apeldoorn
}




\section{Promotor}

Prof. dr. H. ten Cate

\section{Copromotores}

Dr. B. de Laat

Dr. J.A. Remijn (Gelre Ziekenhuizen, Apeldoorn; Meander Medisch Centrum, Amersfoort)

\section{Beoordelingscommissie}

Prof. dr. J.W.M. Heemskerk (voorzitter)

Dr. F.M.E. Franssen

Dr. ir. Y. Henskens

Prof. dr. M.P.M. de Maat (Erasmus Medisch Centrum, Rotterdam)

Prof. dr. J.C.M. Meijers (Universiteit van Amsterdam, Amsterdam) 


\section{CONTENTS}

$\begin{array}{lll}\text { Chapter } 1 & \text { General introduction and outline } & 7\end{array}$

Part I Detection of active VWF by a VHH: mechanisms and assay validation

Chapter 2 A variable heavy chain antibody against active von Willebrand 45 factor only prevents platelet-VWF binding in solution

Chapter 3 Analytical characterization and reference interval of an enzymelinked immunosorbent assay for active von Willebrand factor

Part II The effect of repeated strenuous exercise on active VWF

Chapter 4 Effects of repeated bouts of exercise on the haemostatic system 91

Chapter 5 A hypoxic environment attenuates exercise-induced procoagulant 121 changes due to decreased platelet activation

Part III Active VWF in pathologies associated with disbalanced haemostasis

Chapter 6 Acute exacerbations of COPD induce a prothrombotic state 151 through platelet-monocyte complexes, endothelial activation and increased thrombin generation

Chapter 7 Circulating active von Willebrand Factor levels are increased in chronic kidney disease and end-stage renal disease

Chapter 8 Active von Willebrand Factor in patients with a bleeding diathesis a narrative review

Chapter 10 Summary, general discussion and future prospects

Chapter 11 Nederlandse samenvatting

Appendices

Appendix I Valorisation

Appendix II Curriculum Vitae 


$$
\text { Chapter } 1
$$




\section{General introduction and outline of the thesis}





\section{GENERAL INTRODUCTION}

\section{Haemostasis: a balancing act}

Haemostasis, the cessation of blood loss from a damaged vessel, is an extremely important physiological process that is highly conserved among species. ${ }^{1}$ Haemostasis is accomplished by a complex set of reactions that involve three main components: the blood vessel lining (the endothelium), platelets and plasma (anti-)coagulation proteins. $^{2-4}$

Under physiological conditions, the endothelium has anticoagulant properties and keeps blood in its fluid state. ${ }^{5}$ However, upon injury to the vasculature, blood is exposed to the subendothelial matrix, which contains several components that activate the two main processes of haemostasis: platelet plug formation and fibrin deposition. These processes are tightly regulated, in order to respond rapidly but remain localized to the site of injury. ${ }^{2}$ The coagulation process, according to the currently prevailing cell-based model $^{6}$, occurs in three overlapping stages, as outlined in more detail below.

\section{Initiation}

The initiation phase begins when the vessel wall is damaged and subendothelial cells, such as smooth muscle cells and fibroblasts, are exposed to flowing blood. ${ }^{7}$ These cells expose TF, which binds to factor (F)VIla that is present in the blood. The TF/FVIla complex cleaves FIX and FX into FIXa and FXa, respectively. FXa can activate more FVII to FVIla, accelerating the start of coagulation. In the absence of cofactor FVa, FXa alone can produce trace amounts of thrombin from prothrombin (Figure 1, left panel). ${ }^{2}$

\section{Amplification}

In the amplification phase the procoagulant action moves to the platelet surface. The thrombin that is already formed activates platelet-bound FV, FVIII and FXI (Figure 1, right panel). The coagulation response is amplified as platelets adhere to the denuded endothelium, which not only exposes TF-bearing cells but also collagen. Platelets contain several receptors to adhere to collagen. ${ }^{8}$ Under low shear conditions ( 250-500 $\mathrm{s}^{-19}$ ), platelets bind collagen via the platelet integrin $\alpha 2 \beta 1$ (glycoprotein (GP)la/lla) and GPVI receptors. ${ }^{10}$

At high shear rates $\left(>1,500 \mathrm{~s}^{-1}\right)$, as in the arterial circulation, the multimeric protein von Willebrand factor (VWF) plays a crucial role in platelet adhesion by acting as a molecular bridge between the platelet GPIb-IX-V complex and collagen. ${ }^{11}$ The size of the VWF multimers is important for this function and is regulated by the protease ADAMTS13 (a disintegrin and metalloproteinase with a thrombospondin type 1 motif, member 13). ${ }^{12,13}$ 

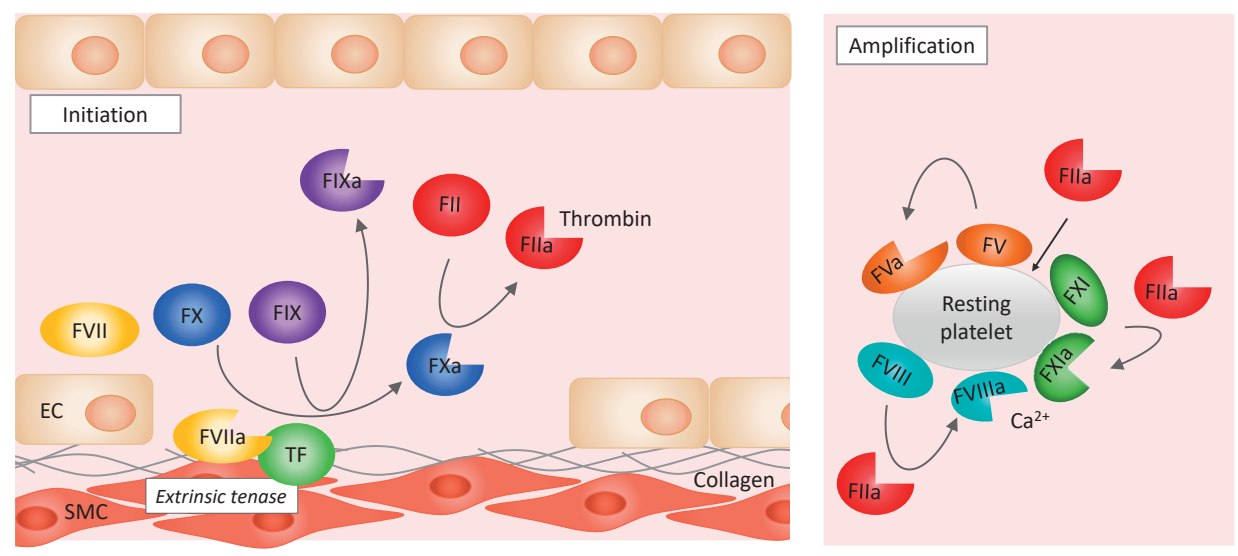

Figure 1. The initiation and amplification phases of the coagulation process. (A) Upon vessel wall injury, tissue factor (TF) is exposed to blood and binds to FVlla. The TF-FVIla (extrinsic tenase) complex activates small amounts of FIX and FX. (B) In the amplification phase, thrombin activates cell-surface bound FV, FVIII, FXI.

Following initial tethering and rolling of platelets, stable adhesion of platelets is required, and this is mediated through additional contacts with the subendothelial matrix or endothelial cells (Figure 2). Binding of GPIb $\alpha$ and GPVI to VWF and collagen, respectively, initiates a complex series of intracellular reactions that result in platelet activation (Figure 3). Activation causes the normally discoid platelets to change shape, forming extrusions from their membrane called filopodia. This shape change makes them extremely adhesive. ${ }^{14}$ Furthermore, activation of platelets induces secretion of their granules into the circulation. Platelets contain two types of granules. Dense granules have CD63, LAMP-2, GPIb and $\alpha$ llb $\beta 3$ on their membrane and contain nucleotides (e.g. ADP, ATP), amines (serotonin, histamine), and several ionic species $\left(\mathrm{Ca}^{2+}, \mathrm{Mg}^{2+}\right.$, $\mathrm{K}^{+}$, polyphosphates). ${ }^{2,8}$ The largest granule population in platelets (50-80/platelet) is represented by the $\alpha$-granules, which contain a number of procoagulant factors (e.g. FV, prothrombin, PAI-1, fibrinogen and VWF), coagulation inhibitors (e.g. tissue factor pathway inhibitor (TFPI), antithrombin (AT), protein S, C1-inhibitor), membrane-bound receptors (e.g. $\alpha$ llb $\beta 3, \mathrm{GPVI}, \mathrm{GPIb}-\mathrm{IX}-\mathrm{V})$, and P-Selectin. ${ }^{15}$

The accumulating thrombin formed during the initiation and amplification phases binds to the platelet protease-activated receptors (PAR)-1 and PAR-4, contributing to strong platelet activation. ${ }^{16}$ In addition, the interaction of the positive feedback mediator ADP (released from dense granules) with platelet $\mathrm{P}_{2} \mathrm{Y}_{1}$ and $\mathrm{P} 2 \mathrm{Y}_{1} 12$ receptors contributes to strong platelet activation, inducing the activation of the $\alpha \mathrm{llb} \beta 3$ receptors on the platelet membrane. ${ }^{17}$ This platelet receptor allows for platelet aggregation, as it interacts with multiple ligands that promote platelet-platelet binding, including VWF and its primary 


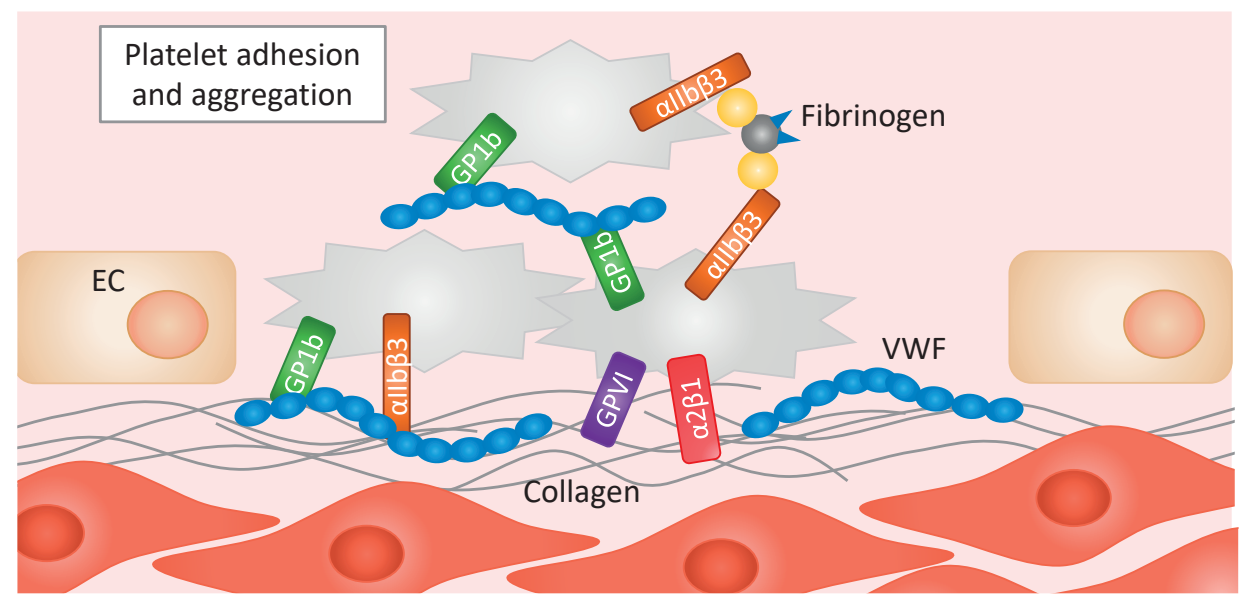

Figure 2. Major platelet receptors and components of the subendothelial matrix involved in platelet adhesion and aggregation. After platelets are activated, they undergo shape changes, producing elongated filopodia, and become extremely adhesive. Under low shear conditions, platelets can bind to collagen via their GPVI and $\alpha 2 \beta 1$ receptors, whereas under high shear stress they depend on VWF to function as a bridge between collagen and the GP1b $\alpha$ receptor. Aggregation of platelets is mediated predominantly by the $\alpha$ llb $\beta 3$ receptor, that binds fibrinogen (and VWF). Abbreviations: VWF, von Willebrand Factor; EC, endothelial cell; GP, glycoprotein.

ligand fibrinogen. ${ }^{18}$ Fibrinogen binds two activated $\alpha$ llb $\beta 3$ receptors simultaneously, and hence acts as a bridge between platelets to facilitate aggregation. ${ }^{19}$

Platelet aggregation also supports thrombin generation (Figure 3). Platelets contain procoagulant phospholipids, predominantly negatively charged phosphatidylserine (PS), on the inner leaflet of their membrane. Upon activation, phosphatidylserine becomes exposed on the outer membrane of a subset of platelets ${ }^{20}$ and serves as a surface for the propagation phase to form a burst of thrombin. ${ }^{21}$

\section{Propagation}

The final propagation phase proceeds on the surface of activated platelets (Figure 4). FIXa associates with thrombin-cleaved FVIIla on the platelet surface, forming the intrinsic tenase complex that catalyses the conversion of FX to FXa. The FXa-FVa prothrombinase complex induces an explosive burst of thrombin generation. Thrombin subsequently cleaves fibrinogen into fibrin, which forms a network that strengthens the clot. Moreover, thrombin activates FXIII to FXIIla (not shown in Figure 4), which in turn crosslinks adjacent fibrin chains to yield an elastic, polymerized fibrin clot. ${ }^{2}$ 


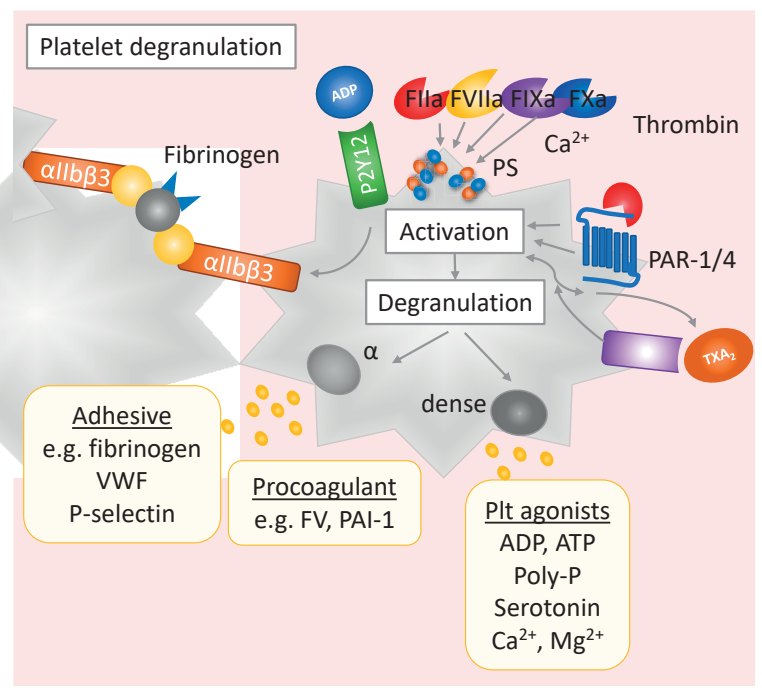

Figure 3. Key platelet receptors and their ligands. Platelet activation through binding of ligands to their receptors induces secretion of alpha $(\alpha)$ and dense granules. Their contents comprise a variety of (anti) coagulant factors and platelet agonists. In addition, activation results in PS exposure and the binding of procoagulant factors to this PS surface, which leads to a burst of thrombin generation. Abbreviations: PS, phosphatidylserine; $F$, factor; ADP/ATP, adenosine di/triphosphate; Poly-P, polyphosphates; VWF, von Willebrand Factor; PAI-1, plasminogen activator inhibitor-1.

\section{Limiting clot growth}

To prevent excess clotting and unrestricted growth of a thrombus, several processes that negatively regulate coagulation are in place. Thrombin does not only boost its own production but also inhibits its generation via the activated protein C (APC) system. ${ }^{22}$ As its concentration gradually rises, thrombin binds to thrombomodulin (TM), a protein expressed on endothelial cells. ${ }^{23}$ TM-bound thrombin then proteolytically activates protein $C$, bound to the nearby endothelial protein $C$ receptor. APC subsequently associates with its cofactor protein $S$ to form a complex that cleaves and hence inactivates

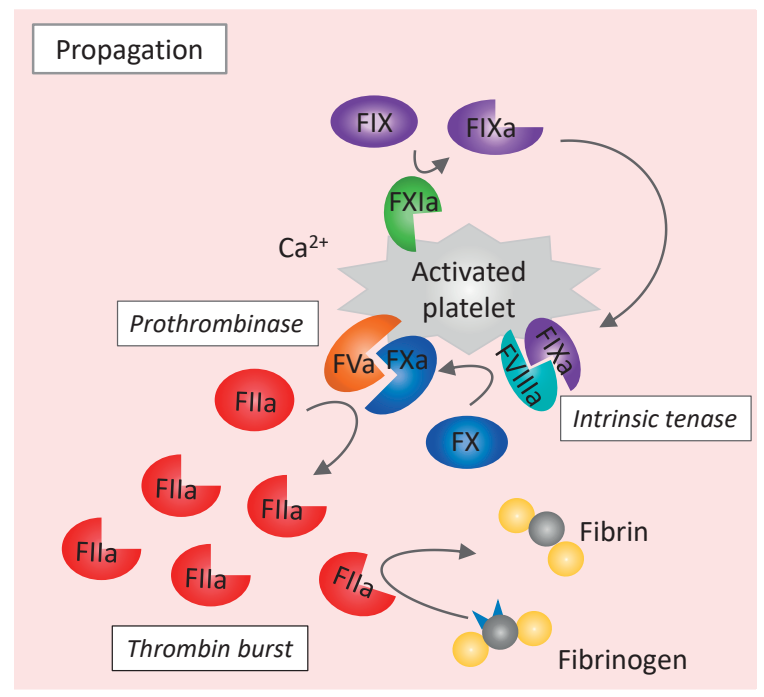

Figure 4. The propagation phases of coagulation. In the propagation phase FIXa binds to FVIIla (intrinsic tenase) on negatively charged phosphatidylserine-rich platelet membranes, activating FX and initiating a burst of thrombin (Flla) generation. Abbreviations: F, factor. 
FVa and FVIIla, thus suppressing prothrombinase and tenase activity (Figure 5). ${ }^{22,24} \mathrm{An}$ other anticoagulant mechanism that dampens clot formation is binding of AT to several coagulation factors, most importantly thrombin and FXa. ${ }^{2}$ Thirdly, TFPI binds to, and thereby inhibits, FXa and the TF/VIIla complex. ${ }^{25}$ Protein $\mathrm{S}$ also acts as a cofactor in the interaction of TFPI with FXa, catalysing inhibition of the latter. ${ }^{26}$

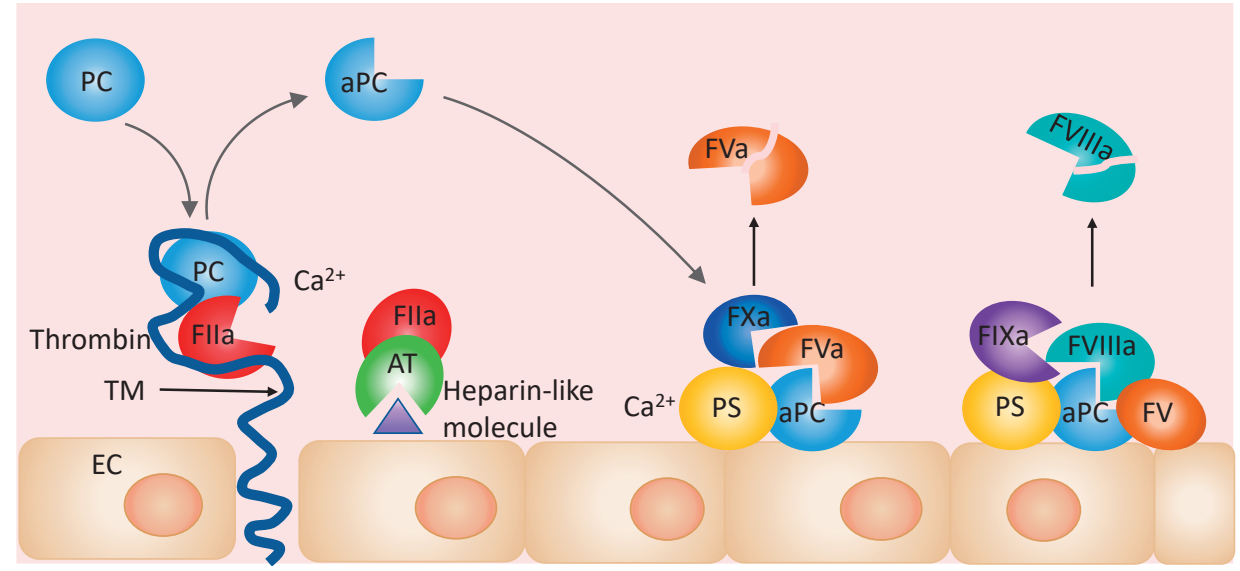

Figure 5 Thrombin-mediated activation of Protein C (PC) and inactivation by AT. Thrombin binds to its receptor thrombomodulin (TM), together with protein $C$ (PC), allowing for its cleavage and activation into APC. APC inactivates both FVa and FVIIla. This results in loss of active tenase (FIXa/ FVIIla) and prothrombinase (FKa/FVa) complexes.

\section{Fibrinolysis}

Besides limiting clot growth, degradation of the fibrin clot (called fibrinolysis), is crucial to maintain a balance in haemostasis. Plasmin is the key protease of the fibrinolytic system, cleaving fibrin into fibrin degradation products (FDPs) to break down the clot (Figure 6). During clot development, plasmin is generated from plasminogen. This conversion is mediated by tissue-type plasminogen activator (tPA), released from endothelial cells, and to a lesser extent, by urokinase-type plasminogen activator (UPA), secreted from macrophages, renal epithelium and endothelial cells. ${ }^{27}$ In fact, fibrin regulates its own degradation by binding both plasminogen ${ }^{28}$ and $\mathrm{tPA}^{29}$ on its surface, thereby localizing and enhancing plasmin generation. Another amplification mechanism of fibrinolysis is plasmin cleavage of both TPA and UPA, transforming them from a single chain to more active two-chain polypeptides, thereby forming a positive-feedback loop. ${ }^{27}$ Of note, plasmin does not only cleave fibrin but also targets many other coagulation factors, ${ }^{30-32}$ VWF as well as the VWF cleaving protease ADAMTS13. ${ }^{33}$

Similar to coagulation, fibrinolysis is also negatively regulated by several inhibitors. First of all, tPA and uPA are directly inactivated by the plasminogen activator inhibitors 


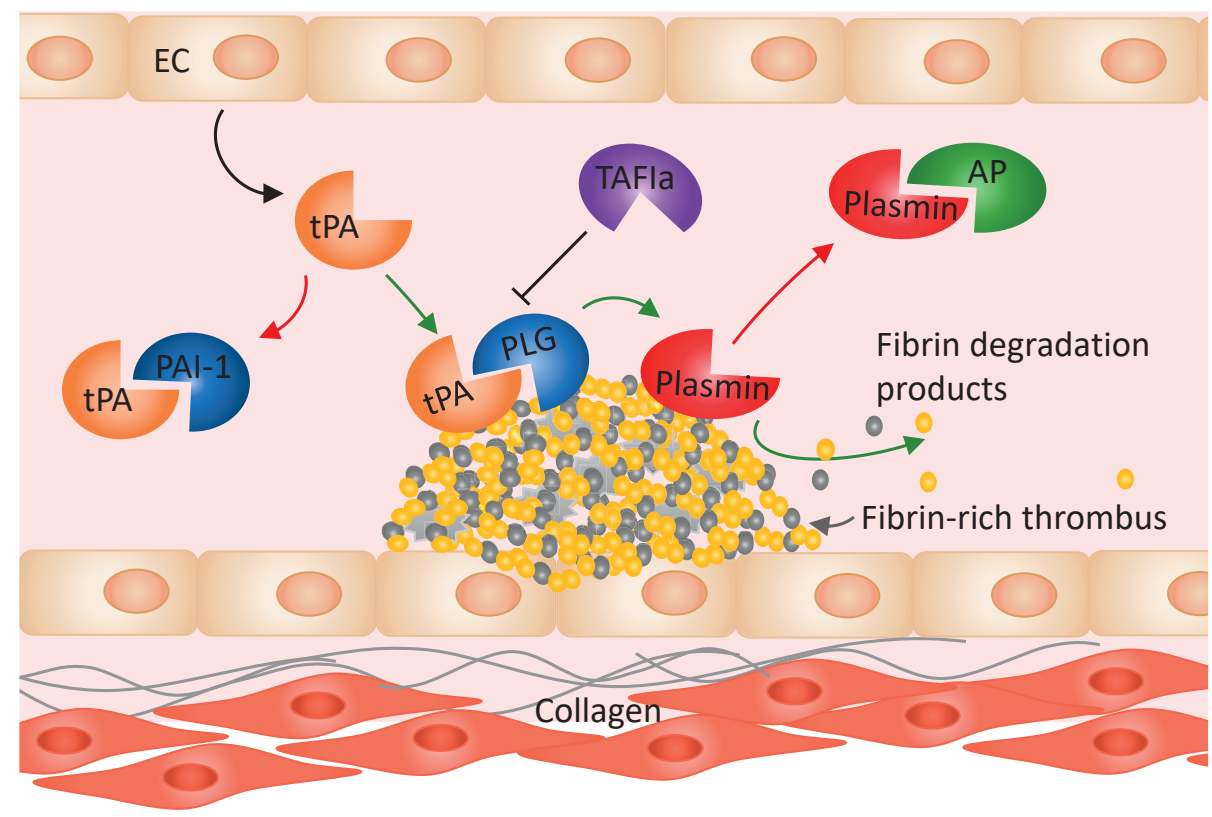

Figure 6. Fibrinolysis. In the absence of fibrin, tissue plasminogen activator (tPA) released by endothelial cells is inactivated by plasminogen activator inhibitor (PAI-1), and these complexes are removed from the circulation via hepatic clearance. When a fibrin-rich clot is formed, tPA and plasminogen (PLG) are bound to fibrin via lysine residues. Plasminogen is converted to plasmin, which digests fibrin into its degradation products. Thrombin activatable fibrinolysis inhibitor (TAFI), activated (a) by thrombin, attenuates fibrinolysis by removal of lysine binding sites on fibrin. Eventually, fibrinolysis by plasmin is limited by antiplasmin (AP).

1 (PAl-1, secreted from activated platelets) and PAI-2. ${ }^{34,35}$ Secondly, plasmin is rapidly inhibited by $\alpha 2$-antiplasmin ( $\alpha 2-A P)$, and to a lesser extent by $\alpha 2$-macroglobulin $(\alpha 2 M)$, unless it remains bound to fibrin or its cell surface receptors. ${ }^{36}$ Thirdly, binding of plasminogen and tPA to fibrin can be blocked by thrombin-activatable fibrinolysis inhibitor (TAFI), thereby limiting plasmin generation. ${ }^{37}$

\section{Pathological haemostasis}

Under physiological conditions, an intricate equilibrium exists between processes and factors that stimulate versus limit coagulation. Disturbances on either side of the balance between coagulation and fibrinolysis can cause thrombosis or bleeding, both of which can be life-threatening.

\section{Thrombosis}

The pathophysiology of thrombosis was first described by Rudolf Virchow in 1856. Virchow postulated that a triad of conditions predispose to thrombus formation (Figure 7), namely abnormalities in (1) the vessel wall (2) blood flow and (3) coagulability of 


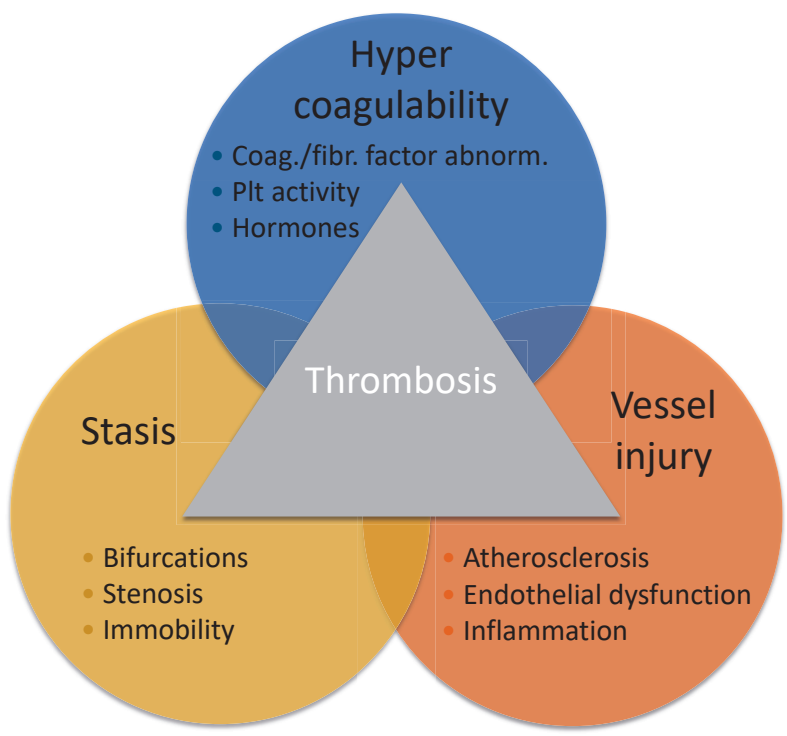

Figure 7. Virchow's triad. The three broad categories of factors that are thought to contribute to thrombosis are hypercoagulability, stasis and vessel injury. Listed under these categories are some examples of general pathologic conditions/causes of disturbance of each specific factor, but many more can be named. Abbreviations: coag, coagulation; fibr, fibrinolytic; abnorm, abnormalities; Plt, platelet.

the blood. ${ }^{38}$ Based on current insights these three components can be further specified. Abnormalities in the vessel wall mainly comprise atherosclerosis, inflammation and endothelial dysfunction. Blood flow is disturbed predominantly at bifurcations and stenotic regions, causing turbulence. Finally, abnormalities in platelet function, levels and activities of coagulation factors and fibrinolytic proteins as well as other plasma proteins can cause hypercoagulability of the blood..$^{39}$

Thrombosis can occur in arteries and veins (Table 1). Arterial thrombi form under conditions of high shear stress. They are typically formed upon rupture of an atherosclerotic lesion, resulting in exposure of the subendothelium and prothrombotic factors, such as $T F$, and subsequent platelet adhesion and aggregation. The growing thrombus causes stenosis, and eventually occludes the blood vessel. Clinical manifestations of arterial thrombosis are myocardial infarction, ischemic stroke and peripheral artery disease. ${ }^{40}$

Venous thromboembolism (VTE) includes deep vein thrombosis (DVT) and pulmonary embolism (PE). In contrast to arterial thrombosis, VTE is not associated with disruption of the endothelial barrier, but with abnormalities in blood flow (stasis) behind venous valves. This leads to activation of the endothelium, which allows for binding of circulating blood cells and initiation of coagulation. ${ }^{41}$ 
Table 1. Main differences in characteristics and mechanism of formation between arterial and venous thrombi.

\begin{tabular}{|c|c|c|}
\hline & $\begin{array}{l}\text { Arterial thrombus } \\
\text { ('white clot") }\end{array}$ & $\begin{array}{l}\text { Venous thrombus } \\
\text { ("red clot") }\end{array}$ \\
\hline Composition & Predominantly platelets & Predominantly RBCs and fibrin \\
\hline Consistency & Firm & Soft \\
\hline Rate of formation & Slow & Fast \\
\hline Mechanism & Turbulence, platelet activation & Low pressure, stasis \\
\hline Treatment & Antiplatelet therapy & Anticoagulation \\
\hline
\end{tabular}

Since arterial thrombi are rich in platelets, they are traditionally referred to as 'white clots'. Venous thrombi, on the other hand, are mainly composed of fibrin and red blood cells. They are therefore commonly referred to as 'red clots'. Although clots are highly heterogeneous in composition, the treatment modality is based on the different compositions of red and white thrombi: antiplatelet agents (e.g. clopidogrel) are the treatment of choice for white arterial thrombi, whereas red venous thrombi are better treated with anticoagulation (e.g. direct oral anticoagulants or heparin). ${ }^{42}$

\section{Bleeding}

When the haemostatic balance tilts towards a hypocoagulable state, a stable blood clot cannot be formed, and bleeding occurs. Bleeding disorders can be caused by defects at all levels of the coagulation process, such as platelet function defects, deficiencies or loss of function of VWF and/or other coagulation factors. They can be congenital, i.e. caused by genetic mutations that induce an abnormal (amount of) protein, or acquired, usually caused by the formation of antibodies against a specific protein. ${ }^{43}$ The extent to which haemostasis is disturbed partly determines the severity of the bleeding phenotype. For instance, haemophilia A and B are caused by severe FVIII and FIX deficiencies, respectively, and are associated with major bleeding episodes. ${ }^{44}$ Platelet secretion defects, on the other hand, usually result in more mild bleeding such as epistaxis and menorrhagia. ${ }^{45}$ The most frequent inherited bleeding disorder is von Willebrand disease (VWD), with a prevalence of up to $1 \%$ based on epidemiological studies. ${ }^{46}$ VWD is caused by deficient (type 1 and 3) or defective (type 2) VWF protein, and will be described in more detail later in this chapter. Clinically, VWD is characterised by excessive mucocutaneous bleeding, such as epistaxis, menorrhagia and prolonged bleeding after trauma. ${ }^{47}$

\section{VWF: a protein with two faces}

The clinical phenotype of VWD exemplifies that VWF is of critical importance for the cessation of bleeding upon damage to the vasculature. VWF deficiency and dysfunc- 
tional VWF, as in VWD, are associated with a bleeding tendency. Paradoxically, bleeding can result from mutations that cause either an increased or a decreased affinity of VWF for platelets. ${ }^{48}$ Conversely, elevated plasma levels of the VWF-FVIII complex represent a dose-dependent risk factor for both arterial and venous thrombosis. ${ }^{49}$ This two-faced character of VWF makes it a complex but highly interesting protein to study. Before describing the role of VWF in the pathophysiology of several bleeding- and thrombotic disorders, more details about the synthesis, functionalities and clearance of VWF are described below.

\section{VWF structure}

VWF, a sialoglycoprotein composed of multimers, is the largest protein in the human circulation. ${ }^{50}$ VWF is synthesized primarily by endothelial cells, but also by megakaryocytes, the precursor cells of platelets that reside in the bone marrow. ${ }^{51}$ The primary product of the VWF gene, located on chromosome 12, is a 2813-amino acid protein consisting of a signal (pre-)peptide (SP), a large propeptide and a mature VWF molecule of $270 \mathrm{kDa}$ (Figure $8 \mathrm{~A}$ ). ${ }^{50}$ Together, the propeptide and mature VWF form the pro-VWF monomer, which comprises four types of homologous domains, in order from $\mathrm{N}$ - to $\mathrm{C}$ terminus: D1-D2-D'-D3-A1-A2-A3-D4-C1-C2-C3-C4-C5-C6-CK (Figure 8B). The D1-D2 domains represent the propeptide, while the remainder corresponds to the mature VWF subunit. $^{52}$

The VWF domains each contain binding regions with specific haemostatic functions (Figure $8 \mathrm{C}$ ). The D3 domain is involved in the $\mathrm{N}$-terminal multimerization of VWF repeating subunits (called protomers) ${ }^{54}$ and is a binding site for FVIII. ${ }^{55}$ The A1 domain binds platelet receptor GPIb $\alpha$ as well as heparin and collagen type VI. ${ }^{56-58}$ ADAMTS13 binds to the VWF A2 domain, where it cleaves the Tyr1605-Met1606 peptide bond. ${ }^{59,60}$ The A3 domain binds to fibrillar collagen types I and III. ${ }^{61}$ The C-terminal C domains contain a sequence that is recognized by activated platelet $\alpha$ llb $\beta 3 .{ }^{18}$

\section{Biosynthesis and storage of VWF}

Following translation, the signal peptide directs pre-pro-VWF to the endoplasmic reticulum (ER) (Figure 9). In the ER, the signal peptide is cleaved off, extensive N-linked glycosylation occurs and C-terminal dimers (protomers) are formed. ${ }^{62}$ These pro-VWF dimers are then translocated to the Golgi compartment, where O-linked glycans are added and $\mathrm{N}$-linked glycans are sulphated. In the trans-Golgi network, $\mathrm{ABO}(\mathrm{H})$ blood group sugars are added to $\mathrm{N}$-linked glycans and $\mathrm{N}$-terminal multimerization via disulphide bridges takes place. ${ }^{63}$ This multimerization process generates a variety of differentially sized multimers composed of up to 250-3000 monomers. ${ }^{64}$ Moreover, VWF propeptide (VWFpp) is cleaved off by furin. ${ }^{65}$ 
A

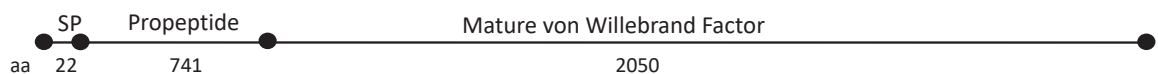

B

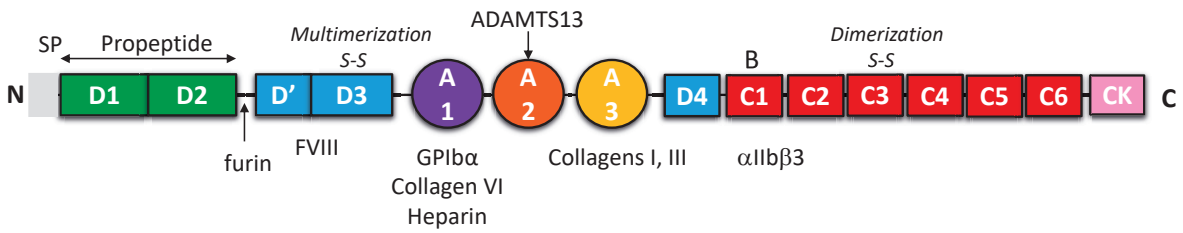

C

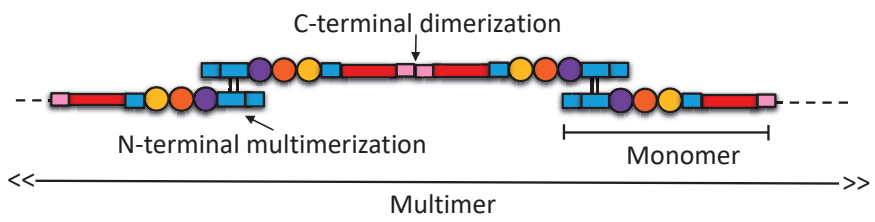

Figure 8. VWF structure. (A) VWF translation product, showing the signal peptide (22 aa), 741 aa propeptide and 2050 aa mature protein. (B) VWF domain annotation, with cleavage sites for furin, ADAMTS13 and binding sites for FVIII, GP1b $\alpha$, collagens and platelet receptor $\alpha$ llb $\beta 3$ indicated. (C) Monomeric VWF dimerizes at the C-terminal CK domain to form the repeating unit of multimeric VWF. Multimers assemble via the formation of N-terminal disulphide bonds between D3 domains. Partly adapted from van der Vorm et al. ${ }^{53}$, see Chapter 3.

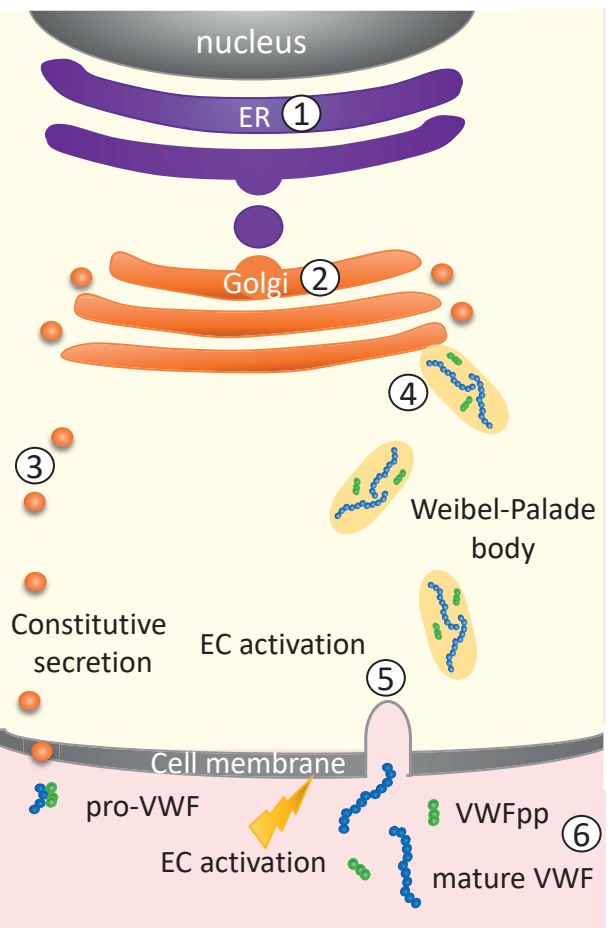

Figure 9. Synthesis of VWF by endothelial cells. (1) After translation, pre-pro VWF is a C-terminal bonded dimer in the endoplasmic reticulum (ER), where the signal peptide is cleaved off. (2) In the Golgi N-linked multimerization occurs and the VWFpp is cleaved off from mature VWF by furin. (3) A small amount of pro-VWF (low molecular weight VWF containing the propeptide), is secreted by the conventional constitutive pathway. (4) Both VWFpp and mature VWF can be sequestered into Weibel-Palade bodies for storage. (5) Although most WPB contents leave the endothelial cell by basal secretion, the ultra large VWF multimers that support platelet recruitment after vascular damage are secreted after activation of the endothelial cell (regulated release). (6) Outside the endothelial cell VWFpp and VWF dissociate. In megakaryocytes/platelets a similar biosynthesis process occurs but VWF is stored in $\alpha$-granules, which also contain many other proteins and hence also form in the absence of VWF. 
In endothelial cells, mature VWF and VWFpp are either constitutively secreted into the plasma (mostly low-molecular-weight multimers) or sequestered within specific organelles known as Weibel-Palade bodies (WPBs). ${ }^{66}$ This WPB-stored VWF comprises predominantly high-molecular-weight multimers, as well as VWFpp. WPB contents are released from endothelial cells through both basal and regulated mechanisms. Whereas the majority of plasma VWF is thought to originate from basal secretion, the ultra-large (>10,000 kDa) endothelial cell-anchored VWF multimers (UL-VWF) that recruit platelets are observed upon endothelial stimulation by thrombotic or inflammatory stimuli, and are hence derived from regulated release. ${ }^{67,68}$ Of note, both mature VWF (VWF antigen) and VWF propeptide serve as markers of endothelial cell activation, although VWF propeptide reflects acute endothelial cell activation because of its shorter half-life. ${ }^{69}$ Following the release of VWF into the blood, the metalloproteinase ADAMTS13 cleaves the A2-domain of VWF to generate smaller VWF multimers in the range of 1-20 dimers/ protomers. ${ }^{12,13}$ Circulating VWF can also be proteolytically cleaved by other enzymes, such as plasmin. ${ }^{70,71}$

In megakaryocytes/platelets, VWF is transported to $\alpha$-granules. Approximately $15 \%$ of total VWF is produced by platelets/megakaryocytes and stored in their $\alpha$-granules, but very little is thought to be secreted into the circulation until platelet activation. ${ }^{64}$ Of note, $\alpha$-granules also form in the absence of VWF, but the formation of WPBs is strictly VWF-dependent. ${ }^{72}$

\section{VWF glycosylation and clearance}

VWF, like all other plasma proteins, has a limited lifespan in the circulation, which ensures that "old" protein is removed timely. The half-life of endogenous VWF is highly variable between individuals, ranging from 4.2 to 26 hours. ${ }^{73}$ Inter-individual differences in VWF glycosylation pattern are likely an important factor determining the lifespan of VWF. Both $\mathrm{N}$ - and O-linked glycans of the mature VWF can carry $\mathrm{ABO}(\mathrm{H})$ blood group carbohydrates ${ }^{74,75}$, which affect the rate of clearance: in individuals with non-O blood group, VWF has a longer half-life than in individuals with blood group $0 .^{76}$ This results in a $\sim 25 \%$ lower VWF levels in blood group 0 than in non-O individuals. ${ }^{77}$ Importantly, whereas only a fraction of glycans expresses $\mathrm{ABO}(\mathrm{H})$ determinants, the vast majority (>90\%) are capped by negatively charged sialic acid residues, which affect clearance through a number of pathways, as discussed below. ${ }^{78}$ Interestingly, platelet-derived VWF has a different glycosylation pattern than endothelial VWF, with platelet VWF being hyposialylated and lacking the blood group antigen structures. ${ }^{79}$

Concerning the mechanisms of VWF clearance, it is known that the majority of VWF is targeted to the liver, in addition to the spleen. ${ }^{80}$ In particular, macrophages in these tis- 
sues bind and internalize VWF, although other cells may also be involved. ${ }^{81}$ This uptake by endocytosis is mediated by receptors that interact with VWF, depending on its level of sialylation. The first receptor proposed to be important in this context was the hepatocyte Ashwell-Morell receptor (AMR) (Figure 10). Although VWF glycans are usually sialylated, this receptor plays a role in the clearance of hyposialylated VWF, as occurs during e.g. pathogen infections $s^{82}$ and in patients with VWD type $1 .^{83}$ In addition, more recently the macrophage galactose-type lectin $(M G L)$ receptor has been confirmed to play an active role in removing hyposialylated VWF from the circulation. ${ }^{84}$

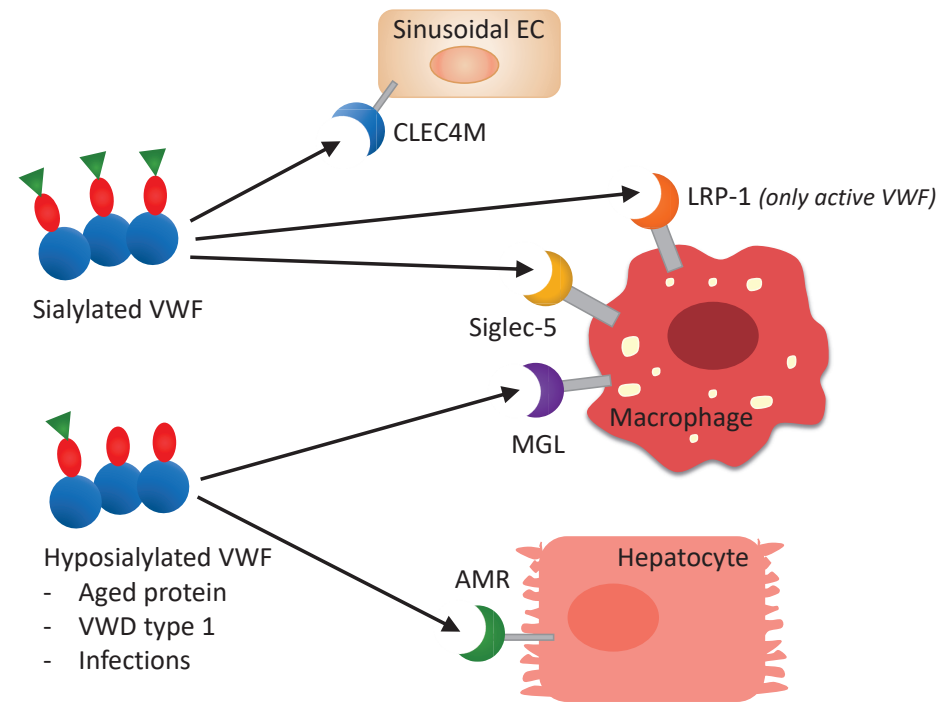

Figure 10. VWF clearance in the liver and spleen. Clearance of VWF (and FVIII, not shown here) occurs in the sinusoids of the liver and spleen, where a range of receptors expressed on macrophages, sinusoidal endothelial cells (EC), and hepatocytes bind and internalize VWF (and FVIII). Protein clearance is influenced by shear (LRP-1 only clears unfolded VWF), desialylation (AMR and MGL receptors specifically clear hyposialylated VWF), and protein sequence variants (affect sialylation, as in VWD type 1). Abbreviations: AMR, Ashwell-Morell receptor; MGL, macrophage galactose-type lectin receptor; LRP-1, LDL-receptor related protein-1.

In contrast, the Siglec- 5 receptor on macrophages specifically interacts with sialic acid residues, and overexpression in a mouse model reduced VWF levels. ${ }^{85}$ Another macrophage receptor involved in VWF (and FVIII) clearance, that is associated with VWF levels in humans, is the lipoprotein receptor LRP1. ${ }^{86,87}$ Importantly, VWF only binds to LRP1 when it is in its unfolded conformation, as occurs under conditions of high shear stress, analogous to the VWF-platelet interaction as described below. ${ }^{88}$ In addition to macrophage receptors, the C-type lectin domain family 4 member M (CLEC4M) receptor, expressed on endothelial cells of liver sinusoids, was also identified to contribute to variation in plasma VWF levels. ${ }^{89}$ 


\section{Haemostatic functions of VWF}

Sufficient levels of functional VWF are crucial for haemostasis, particularly in the rapid flow of the arterioles and at sites of injury. VWF plays dual roles in clot formation (Figure 11). Firstly, VWF mediates the initiation and progression of the primary haemostatic plug, by acting as a molecular bridge between fibrillar collagen of the exposed subendothelial matrix components and the platelet GP1b $\alpha$ subunit of the GPI-IX-V platelet receptor complex. ${ }^{90}$ This transient interaction with collagen-bound VWF is insufficient for platelet arrest, but it does slow down the platelets in the rapidly flowing blood, mediating the "tethering and rolling" of platelets on the endothelial surface. The interaction via GPIb $\alpha$ triggers activation of the platelet $\alpha$ llb $\beta 3, \alpha 2 b 1$ and GPVI receptors, resulting in stable platelet adhesion. ${ }^{91}$ Secondly, VWF is essential for fibrin clot formation, as it assembles with procoagulant FVIII in the circulation, thereby protecting it from rapid clearance. ${ }^{92}$ By acting as its carrier protein, VWF also localises FVIII to the site of vascular injury, where FVIII is important to support secondary haemostasis (evidenced by the bleeding phenotype in haemophilia A patients) after the formation of the initial platelet plug. ${ }^{93}$

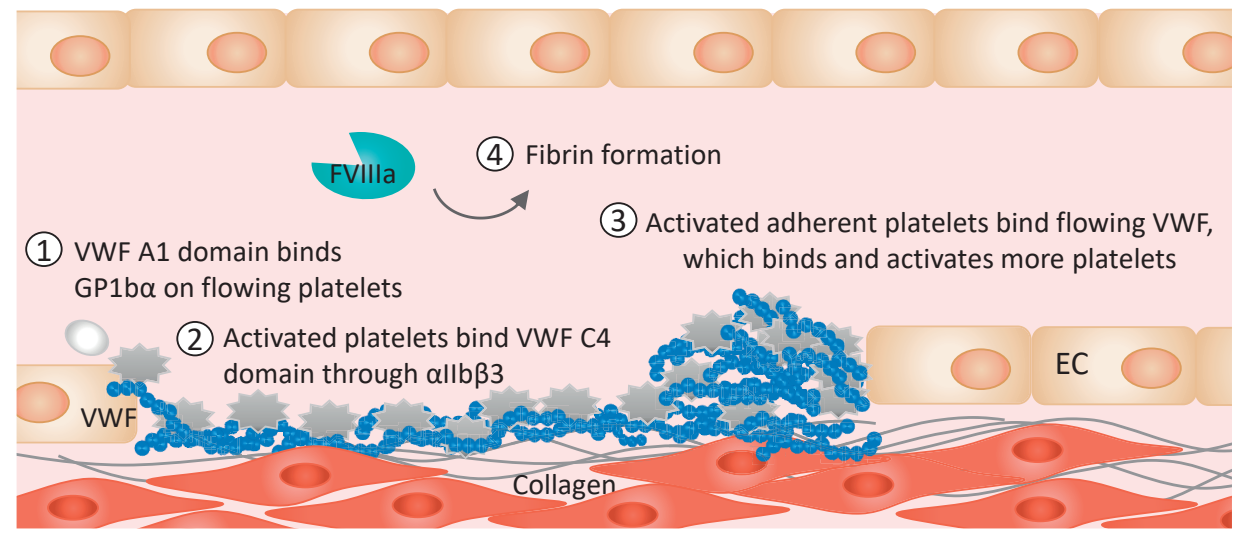

Figure 11. Roles of von Willebrand factor in thrombus formation. At the site of vessel injury, VWF is first immobilised on exposed collagen. (1) Subsequently, flowing platelets bind to the VWF A1 domain via the GP1b $\alpha$ receptor. (2) Clot formation progresses as binding to VWF activates the platelets, so that they can bind more circulating VWF, (3) which then allows additional platelets to be recruited. (4) Tissue factor expressed by subendothelial cells (not shown) initiates secondary haemostasis, supported by co-localised FVIII released from VWF. The resulting fibrin mesh stabilizes the VWF-platelet plug (not shown).

\section{VWF function depends on shear stress and multimer size}

A crucial aspect of VWF function is that, under physiologic conditions, VWF does not spontaneously interact with (non-activated) platelets. ${ }^{56}$ Circulating VWF is normally organized in a compact globular conformation (Figure 12, left)..$^{94}$ This folded conformation 


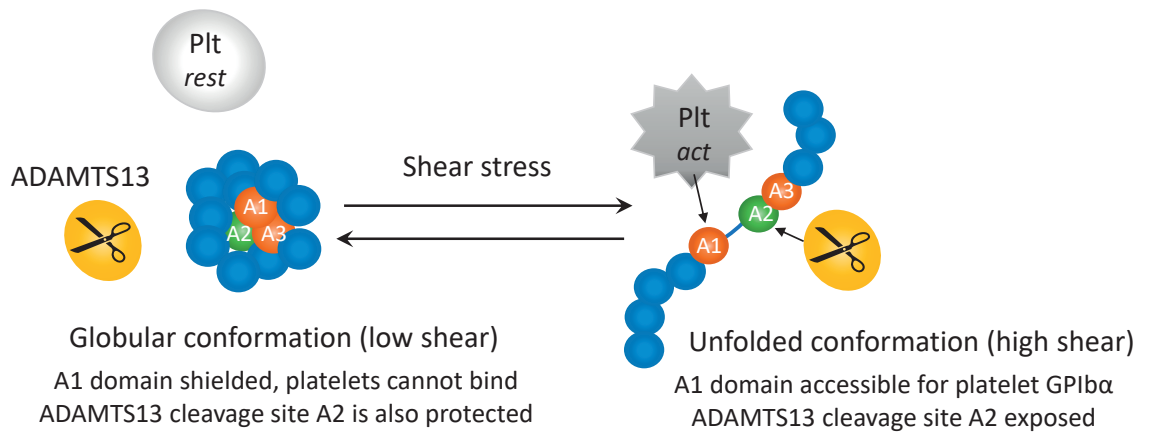

Figure 12. Unfolding of VWF under influence of shear stress. Roughly three states of VWF can be distinguished: fully folded globular structure (left) in which the A-domains are shielded, partially folded globule with an unfolded "tail" (not shown) and fully unfolded linear structure (right), exposing the A1 domain for platelet binding and the A2 domain for cleavage by ADAMTS13. The existence and stability of each of these states are dependent on the VWF multimer size and shear rate.

shields access to the A1 domain, possibly through steric hindrance by O-linked glycosylation ${ }^{95}$ or modulation by the $\mathrm{N}$ - and $\mathrm{C}$-terminal flanking regions of the $\mathrm{A} 1$ domain. ${ }^{96}$ However, VWF is unfolded by either immobilization (via the $A 3$ domain) to subendothelial collagen at sites of vascular injury or elevated shear stress (as in stenosis, associated with shear rates of 3,000-10,000 s${ }^{-1}$ )(Figure 12, right). ${ }^{97,98}$ This conformational shift has functional consequences for the haemostatic potency of VWF. The transition to an elongated "active" conformation exposes the A1 domain, allowing platelets to bind via GPIb $\alpha$, slow down and roll over the VWF surface and ultimately adhere and aggregate.

In addition, the shear-induced opening of VWF allows for its self-association: multimeric VWF binds to other VWF multimers, so that more than one VWF molecule can bind to a single platelet Gplba receptor, promoting clot formation. ${ }^{99}$ Of note, shear rates are normally maximal (up to 3,000 s${ }^{-1}$ ) near the vessel wall (compared to $\sim 200-300 \mathrm{~s}-1$ in the centre of the arterial lumen ${ }^{100}$ ), hence the probability of VWF extending to its unfolded, active conformation is highest where activated VWF is actually required for tethering platelets to the subendothelial matrix (Figure 13). ${ }^{97} \mathrm{All}$ in all, the shear rate at the site of vascular damage is an important determinant for the platelet-binding capacity of VWF in vivo. In vitro, modulating agents, most notably the antibiotic ristocetin, can be used to mimic the effects of shear-induced VWF-platelet binding. Similar to shear stress, ristocetin induces allosteric changes that expose the platelet binding site in the VWF A1 domain. ${ }^{101}$

Besides the shear rate, multimer size also affects the haemostatic activity of VWF, with larger VWF multimers being more haemostatically potent. In contrast to circulating 


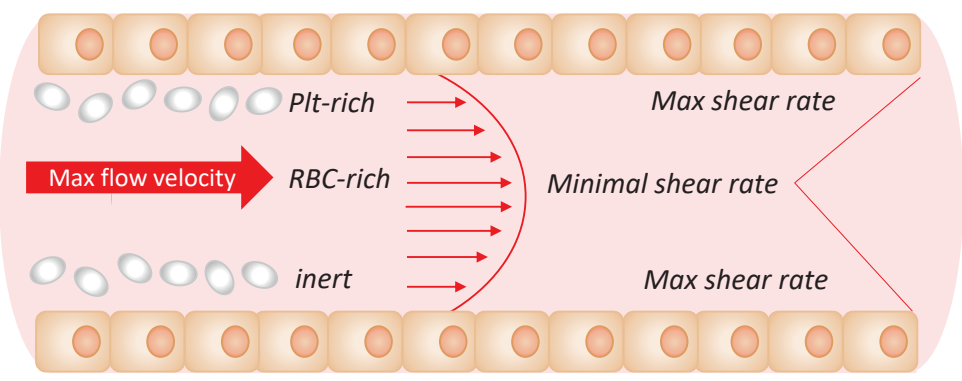

Figure 13. Blood flow rheology and effects on the distribution of blood cells. Normal endothelial cells do not interact with non-activated platelets (grey ovals). Blood flow in a vessel is laminar: it constitutes layers that each move at a different velocity (arrows), with laminae in the centre of the vessel moving at higher velocity than those near the wall. The shear rate is the rate of change of velocity over the distance measured at a 90 degrees angle to the direction of flow. Because of these blood flow properties, the centre of the lumen is red blood cell (RBC)-rich, and they push leukocytes and platelets to the periphery of the vessel, close to the vessel wall.

plasma VWF, UL-VWF freshly released from endothelial cell WPBs can form spontaneous high-strength bonds with platelet GPIb $\alpha .{ }^{102}$

Its hyperreactivity with platelets can be explained by the large size of UL-VWF, with a higher number of platelet and collagen-binding sites ${ }^{103}$, but also by its active, unfolded conformation that directly allows platelet binding. ${ }^{102}$ UL-VWF is thus very potent and potentially dangerous in facilitating undesired platelet aggregation. Therefore, it is normally rapidly processed into smaller less reactive molecules by ADAMTS13. ${ }^{104}$

Unlike most enzymes involved in coagulation, ADAMTS13 is constitutively active in plasma. ${ }^{12,13}$ However, its cleavage site in the A2 domain of VWF is normally buried in the folded VWF molecule, making this globular VWF practically resistant to proteolysis by ADAMTS13. ${ }^{105}$ Similar to the regulation of platelet binding to the A1 domain, VWF proteolysis is regulated by shear-induced conformational changes of VWF, exposing the scissile bond. ${ }^{106}$ After secretion into the circulation, UL-VWF is spontaneously unfolded, in response to the high shear forces of the flowing blood, allowing access for ADAMTS13 to its cleavage site in the A2 domain. Cleavage of the A2-domain of VWF is also influenced by other factors. For instance, the $A 1$ domain inhibits proteolysis of the A2 domain, but binding of platelet Gplba to the A1-domain abrogates this inhibitory effect. ${ }^{107}$ Following proteolysis by ADAMTS13, the multimers are smaller and adopt a globular conformation under normal circulating shear forces, thereby concealing the interaction sites for ADAMTS13 and platelets again. ${ }^{106}$

Together, these shear stress- and size-dependent regulatory mechanisms of the haemostatic activity of VWF ensure that only a small fraction of circulating VWF is in its active, 
platelet-binding conformation. ${ }^{53,108}$ When needed, upon vascular injury, VWF binds to collagen, rapidly unravels and tethers flowing platelets to the vessel wall to initiate platelet-plug formation. However, also several pathological conditions have been identified in which levels of circulating active VWF are increased, leading to premature and/ or excessive formation of VWF-platelet aggregates. ${ }^{109}$

\section{Pathologically elevated active VWF}

Undesired activation of VWF occurs in a variety of pathological conditions. As a result, premature and/or excessive VWF-platelet aggregations are formed, resulting in thrombocytopenia (and consequently increased bleeding risk), thrombotic complications, or both. Overall, three situations disturb the balance between latent and active VWF and may hence lead to increased circulating active VWF:

(1) Altered conformation of the VWF protein, due to:

a. Genetic mutations

b. Increased shear stress

(2) Decreased proteolysis/clearance of active (UL-)VWF from the circulation

(3) Increased secretion of UL-VWF

Elevated active VWF levels have been described in the literature for a number of common as well as rare conditions. A selection of these conditions is described below, grouped according to the three situations described above.

\section{Altered conformation - genetic mutations}

The overall structure and conformation of VWF influence the rate of proteolysis by ADAMTS13 as well as its ability to bind platelets. ${ }^{110}$ This is illustrated by VWD, with 3 main types being distinguished based on quantitative or qualitative defects in VWF. Low VWF protein levels are decreased in VWF type 1, whereas VWD type 3 is characterized by a virtually complete deficiency of VWF. In contrast, VWD type 2 is hallmarked by qualitative defects, and is subdivided into type $2 \mathrm{~A}, 2 \mathrm{~B}, 2 \mathrm{M}$ and $2 \mathrm{~N}$. ${ }^{111,112}$

The most interesting type of VWD with regard to active VWF levels is type $2 \mathrm{~B}$, caused by the presence of gain-of-function mutations (mostly single amino acid substitutions) in exon 28 of the VWF gene, encoding for the A1-domain of VWF. ${ }^{113,114}$ These mutations induce a conformational change in the VWF protein, causing an enhanced affinity of VWF for platelet GP1b $\alpha .{ }^{115}$ The defect induces the spontaneous binding of type 2B VWF to platelets, even in the absence of endothelial cell injury or high shear stress. ${ }^{115}$ In addition, its active conformation renders type $2 \mathrm{~B}$ VWF more sensitive to proteolysis by ADAMTS13. ${ }^{116}$ Counterintuitively, VWD type $2 \mathrm{~B}$ gain-of-function mutations do not result in a thrombotic- but in a bleeding diathesis. Together, premature platelet-VWF 
interaction and increased ADAMTS13-mediated proteolysis result (in most patients ${ }^{117}$ ) in loss of the large, haemostatically most potent VWF multimers and a variable degree of thrombocytopenia. ${ }^{118}$ Levels of active VWF were previously measured in type $2 \mathrm{~B}$ VWD patients and were found to be increased but vary considerably, with a 2 to 15 -fold increase compared to healthy controls. ${ }^{108}$ Moreover, platelet counts correlated inversely with the amount of active type $2 \mathrm{~B}$ VWF. ${ }^{108}$ Thus, the active VWF levels in type 2B VWD patients seem to determine, at least in part, the extent of thrombocytopenia, and hence, the bleeding risk. ${ }^{109,118}$

\section{Altered conformation - increased shear stress}

VWF can transition from its globular to its active conformation as a result of increased shear stress, as is the case in stenotic blood vessels caused by atherosclerotic plaque formation. The role of active VWF in coronary artery disease was first studied in women with acute myocardial infarction before the age of 45 years. This study found increased levels of active VWF in approximately one-third of women with acute myocardial infarction compared to controls, which was not accompanied by ADAMTS13 deficiency. ${ }^{119}$ Another (large-scale) study also found an approximate 1.5-fold increased level of active VWF in patients with first ST-segment elevation myocardial infarction (STEMI) as compared with controls, and again no association between levels of ADAMTS13 and active VWF. ${ }^{120}$ Instead of ADAMTS13 deficiency, it is likely that the high shear stress present in atherosclerotic arteries is responsible for the higher active VWF levels in patients with coronary artery disease.

\section{Decreased proteolysis and/or increased secretion of (UL-)VWF}

The role of (active)VWF in thrombosis is particularly evident in the condition of thrombotic thrombocytopenic purpura (TTP). ${ }^{121}$ Historically, (acute) TTP symptoms have been described by a clinical pentad that included fever, neurological symptoms and renal insufficiency, but the only constant signs of TTP are thrombocytopenia $\left(<30 \times 10^{9} / \mathrm{mL}\right.$ ) and microangiopathic haemolytic anaemia, causing elevated serum LDH levels and schistocytes on the blood smear (due to mechanical destruction of red blood cells in the obstructed microvasculature). ${ }^{122}$ Importantly, patients with another thrombotic microangiopathy, the haemolytic uremic syndrome (HUS), also present with haemolytic anaemia with red blood cell fragmentation, thrombocytopenia and renal failure, but not typically with neurological symptoms. ${ }^{123}$ TTP is caused by a deficiency of ADAMTS13, which can be due to mutations in the ADAMTS13 gene (congenital TTP) ${ }^{124}$ or the presence of autoantibodies (acquired TTP). ${ }^{125}$ The ADAMTS13 deficiency results in failure to cleave the UL-VWF secreted from endothelial cells into the circulation. ${ }^{121}$ These UL-VWF multimers are in their active conformation and therefore interact spontaneously with GP1b $\alpha$ on platelets, leading to the formation of VWF-rich thrombi that occlude the mi- 
crovasculature. ${ }^{121}$ The diagnosis of TTP may be confirmed based on ADAMTS13 activity testing, but this assay is not available in all laboratories and the decision to initiate plasma exchange should not be delayed while the results of this assay are pending. Interestingly, a previous study showed that active VWF levels were not only significantly elevated in TTP patients compared to healthy individuals but also in congenital compared to acquired TTP patients. ${ }^{108}$ Hence, quantification of active VWF levels may potentially be used to distinguish between acquired and congenital TTP.

Besides HUS, another condition with very similar clinical characteristics as TTP is the HELLP (haemolysis, elevated liver enzymes, low platelets) syndrome, which can occur as a complication of preeclampsia during pregnancy. ${ }^{126}$ HELLP manifests clinically with low platelet counts and thrombotic microangiopathy. Active VWF levels were found to be elevated three-fold in pregnant women with HELLP compared to healthy pregnant women. ${ }^{127}$ Mechanistically, the elevated active VWF levels in this disease appear to be caused by a combination of reduced ADAMTS13 levels (and hence reduced clearance of UL-VWF) and increased (total) VWF secretion due to endothelial cell activation. ${ }^{127,128}$

Active VWF also appears to contribute to haemostatic complications of malaria. In healthy volunteers, who were experimentally infected with $P$. falciparum, declining platelet numbers were associated with the onset of endothelial cell activation and with increased active VWF. ${ }^{129}$ Thrombocytopenia and endothelial cell activation were also found in patients with $P$. falciparum and $P$. vivax infection, in addition to reduced ADAMTS13 activity and antigen levels and appearance of UL-VWF. ${ }^{130}$ Dengue is another infectious disease characterized by thrombocytopenia, leading to bleeding and plasma leakage. In dengue patients, activation of the endothelium leads to increased secretion of VWF (and its propeptide) into the circulation, followed by VWF consumption. In addition, an increased amount of VWF was found in its active platelet binding conformation, and concurrently ADAMTS-13 levels were reduced. Circulating platelets were activated, contributing to platelet exhaustion. ${ }^{131} \mathrm{~A}$ more recent study found that VWF also plays an important role in platelet clearance in dengue. Circulating platelets had more VWF bound to GP1b $\alpha$ on their membrane, resulting in the removal of platelet sialic acid and hence platelet clearance and thrombocytopenia. ${ }^{132}$ Together, endothelial cell activation (release of large amounts of active UL-VWF), combined with reduced VWF proteolysis by ADAMTS13 may result in the clinical haemostatic manifestations of these two infectious conditions. ${ }^{130}$

All in all, the above-described findings have provided new insight into the presence of active VWF under several pathological conditions. The origin of the increased amount of circulating VWF in its active platelet-binding conformation differs depending on 


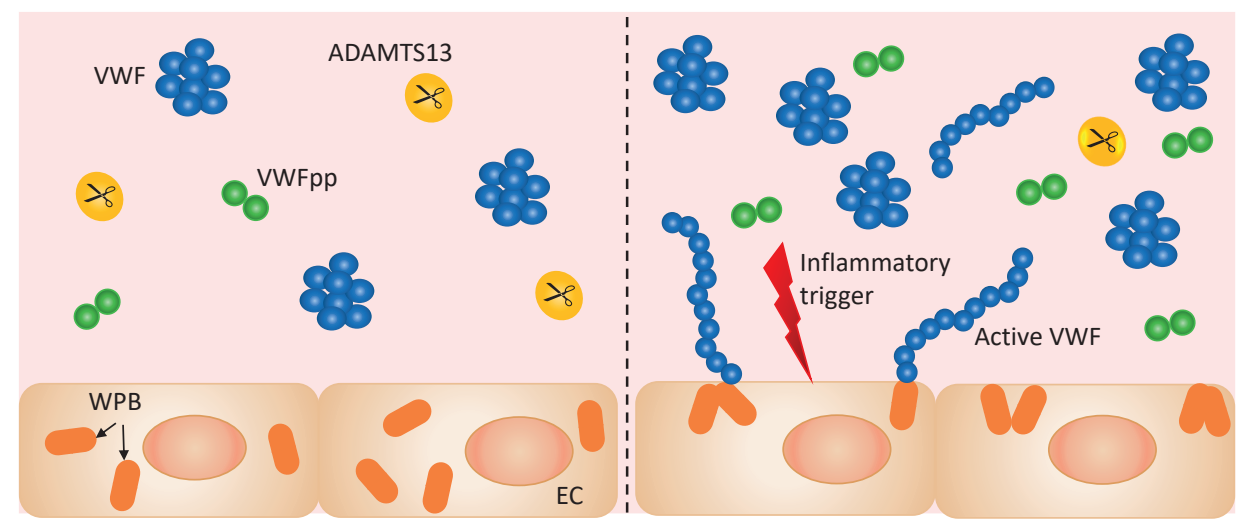

Figure 14. Von Willebrand factor (VWF) as a marker for inflammation. Upon an inflammatory trigger (right), endothelial cells are activated, resulting in the massive release of VWF (globular form) and its propeptide (VWFpp) from endothelial cell (EC) Weibel-Palade bodies (WPBs). Compared to the basal situation (left), VWF and VWFpp levels are therefore increased. In contrast, ADAMTS-13 levels are decreased or less active and as a consequence, VWF in its platelet-binding conformation (active VWF) is also increased. Adapted from Kawecki et al. ${ }^{140}$

the specific condition. Additional studies are needed to unravel the factors/conditions that render VWF active. A common denominator in most non-congenital conditions described above is the presence of inflammatory stimuli. Over the years it has become increasingly recognized that inflammation and coagulation are intricately linked, and VWF is influenced in several ways by inflammatory conditions.

\section{VWF and inflammation}

Besides its important roles in maintaining haemostasis, VWF is a marker of inflammation at the endothelial level. In reaction to an inflammatory stimulus, endothelial cells release the content of their WPBs into the circulation. ${ }^{90}$ The circulating VWF in inflammatory conditions is predominantly of endothelial cell origin; platelets have a relatively low VWF content and platelet VWF is only released upon platelet activation, hence the contribution of platelet VWF to the VWF acute phase response is limited. ${ }^{133}$

Of note, elevated VWF levels are not specific for an inflammatory acute phase response, but are also a marker of vascular dysfunction (Figure 14). To distinguish between an acute and a chronic endothelial disturbance, one can measure both VWF and VWF propeptide (VWFpp) levels ${ }^{134}$. Both proteins are secreted in equal molar concentrations but have different half-lives. ${ }^{135}$ As a result, both VWF antigen and propeptide levels are increased during an acute response of the endothelium (e.g. sepsis). In contrast, VWFpp levels are only marginally increased during chronic stimulation (e.g., diabetes mellitus), while this is accompanied by clearly elevated VWF antigen levels. ${ }^{134}$ Importantly, as 
mentioned for several clinical conditions described above, systemic inflammation has been associated with reduced ADAMTS13 levels ${ }^{136}$ and activity. ${ }^{137,138}$ The latter may be partly explained by the inhibitory effects of proinflammatory cytokines, such as IL-6, on ADAMTS13. ${ }^{139}$ Thus, the increased levels of active VWF in inflammatory conditions can be attributed to increased release of VWF from the endothelium and decreased proteolysis by ADAMTS13.

In addition to being an inflammatory marker, VWF acts as a modulator of inflammatory responses. ${ }^{141,142}$ First, VWF plays an indirect role in the inflammatory process, as VWF is crucial for the formation of WPBs (process 1 in Figure 15). ${ }^{133}$ The release of WPBs in response to an inflammatory stimulus does not only lead to an increase in circulating VWF and VWFpp, but also to the secretion of other pro- and anti-inflammatory mediators present in the WPBs. One of the proteins co-residing with VWF in WPBs is P-selectin. Upon the release of WPBs, P-selectin is exposed to the endothelial surface to serve as a receptor for leukocyte rolling, hence facilitating leukocyte recruitment to the inflamed area. ${ }^{143}$

VWF may also serve as a more direct effector of inflammation, through mediating recruitment of leukocytes to sites of inflammation (see processes 2 and 3 in Figure 15) and regulating vascular wall permeability (process 4 in Figure 15). ${ }^{142,144,145}$ VWF can directly interact with leukocytes. ${ }^{146,147}$ via its $A 1$ domain, which contains a binding site for leukocyte PSGL-1. This is not surprising considering the similarities between leukocyte PSGL-1 and platelet Gplb $\alpha^{146}$ Interestingly, similar to its interaction with platelet $G P 1 b \alpha, V W F$ needs to be in its active conformation to interact with leukocyte PSGL-1. ${ }^{148}$ This implies that particularly active VWF promotes the inflammatory response through leukocyte recruitment. Clinically relevant in this light is a previous study measuring active VWF levels in patients with systemic inflammatory response syndrome. ${ }^{149}$ Remarkably, patients with high active VWF levels on admission were found to have a 2 -fold higher mortality rate. In contrast, total VWF levels were not associated with mortality ${ }^{149}$ supporting the hypothesis that active VWF may be a more useful clinical marker to assess disease severity in patients with inflammatory conditions than total VWF.

\section{Detecting active VWF}

Importantly, for most conditions described above it remains to be established whether the observed increased active VWF levels are causally related to the specific condition or an epiphenomenon of acute disease and inflammation. If a causal relation is established, intervention may become possible. An attractive strategy to this end would be to neutralize the interaction between (active) VWF and platelet GP1b $\alpha$ to prevent thrombus formation and consumption of high molecular weight VWF and platelets. To this end, single-domain antibodies, also called variable heavy chain antibody fragments 


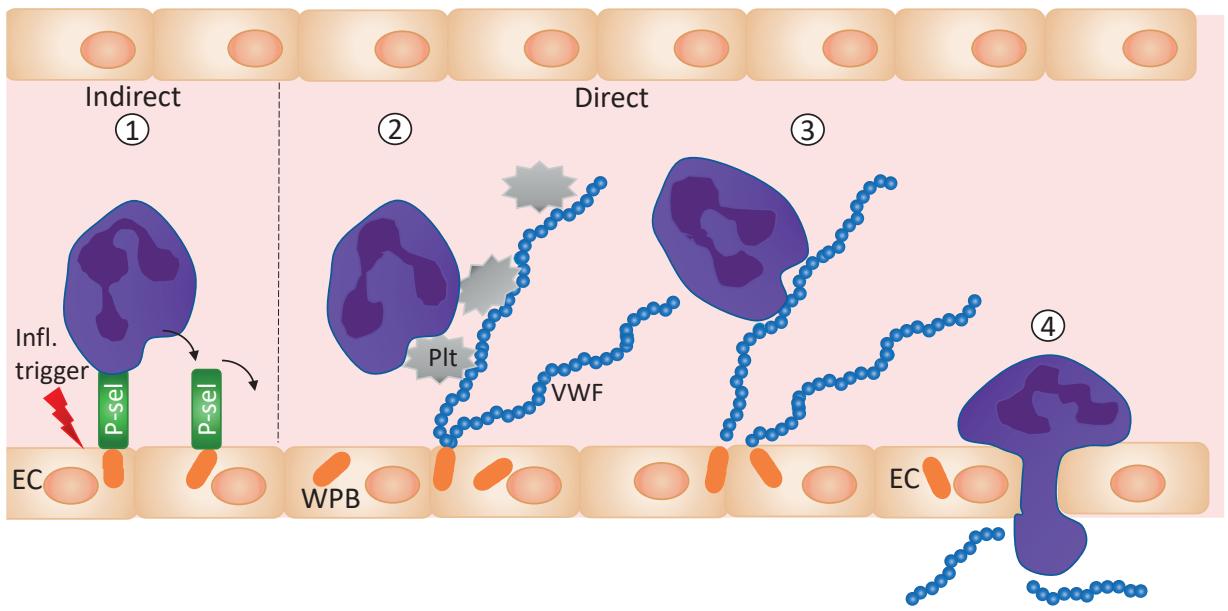

Figure 15. VWF modulates inflammation through indirect and direct mechanisms. (1) An indirect role of VWF in inflammation stems from the fact that VWF is crucial for Weibel Palade body (WPB) formation in endothelial cells (EC). In addition to VWF, these WPBs also contain pro-and anti-inflammatory mediators, which will be released upon an (inflammatory) stimulus. Amongst these is $\mathrm{P}$-selectin (P-sel), which serves as a receptor for leukocyte rolling, an important step in the inflammatory response. (2) VWF released from activated endothelial cells, or bound to collagen at sites of vascular injury, can directly bind platelets via GPIb $\alpha$ and these platelets subsequently enable leukocyte recruitment. (3) VWF can also recruit leukocytes by directly interacting with leukocyte cell surface receptors including $\beta 2$-integrins and PSGL-1. (4) VWF regulates endothelial wall permeability and influences leukocyte (and VWF) extravasation.

(VHHs), against the VWF A1 domain have been developed previously. For instance, the bivalent $\mathrm{VHH}$ caplacizumab targets the A1 domain of VWF. ${ }^{150,151}$ In a recent phase 3 double-blind, randomized controlled trial, 145 patients with TTP received either caplacizumab or placebo during plasma exchange and 30 days thereafter. Caplacizumab treatment reduced the time to normalization of the platelet count, presumably because caplacizumab prevented platelet consumption by microthrombi formation with ULVWF. However, there were also more (serious) bleeding-related adverse events in the caplacizumab-treated group than in the placebo group. ${ }^{150}$ This can be explained by the mechanism of action of caplacizumab, as it blocks all VWF regardless of conformation. In contrast, Hulstein et al. previously reported on a recombinant VHH (AU/VWFa-11) produced in prokaryotic $E$. coli, named AU/VWFa-11, that specifically recognizes the A1 domain of VWF in its active conformation. ${ }^{108}$ Since both this AU/VWFa-11 VHH and Gplba interact with the A1-domain of VWF only upon unfolding, they were hypothesized to share a similar binding site. However, Gplb $\alpha$ and AU/VWFa-11 could interact simultaneously with VWF and AU/VWFa-11 was unable to interfere with the interaction between Gplba and VWF coated to a microtiter plate or VWF bound to collagen under flow. ${ }^{108}$ Given this lack of inhibition of VWF-platelet binding, AU/VWFa-11 was not developed further for potential therapeutic purposes. AU/VWFa-11 was, however, used 
to develop an ELISA assay to quantify plasma levels of active VWF. ${ }^{108}$ This assay was employed in many of the studies described in the previous paragraphs, but was not technically validated and no reference values in healthy individuals were established. 


\section{OUTLINE}

VWF is an incredibly versatile and interesting protein, in particular because of its complex structure-function relationships. This thesis describes our studies with the purpose to further investigate the in vitro (shear stress, collagen-binding) and in vivo (inflammation, hypoxemia) conditions that drive activation of VWF and binding of active VWF to platelets.

The key tool in the "toolbox" of assays that we used to study various components of coagulation, was a single-chain antibody fragment, also called a variable heavy chain antibody $(\mathrm{VHH})$, that was raised against the VWF A1 domain. This $\mathrm{VHH}$, which was given the name S-VWFA1, has a sequence that closely resembles the AU/VWFa-11 VHH described above, but was raised in eukaryotic HEK293E-253 cells instead of prokaryotic E. coli. ${ }^{108}$

Part I of this thesis describes a mechanistic- and an assay validation study using the S-VWFA1 VHH. As this VHH specifically binds to the A1 domain of VWF, which also harbours the platelet-binding site, it can be hypothesized that the $\mathrm{VHH}$ may be able to block VWF-platelet binding. This would be a potentially attractive strategy to prevent pathological platelet-VWF interaction, as occurs in VWD type 2B and TTP. Therefore, the experiments described in Chapter 2 aimed to characterize S-VWFA1 in terms of its interference with platelet-VWF interaction, and the conditions influencing its effects (e.g. shear, collagen), using a variety of functional assays. In Chapter 3, the VHH was used as the capture antibody in an ELISA assay to specifically detect active VWF in plasma samples. We describe the technical validation of this assay, as well as the establishment of a reference interval for active VWF based on values obtained for 120 healthy individuals.

Circulating VWF levels are known to vary considerably between individuals. One of the non-heritable factors that strongly increase VWF levels in healthy individuals is strenuous exercise, which induces endothelial activation. Less is known on the effects of exercise on the active fraction of VWF, hence this gap in knowledge is addressed in Part II. A thorough literature search was performed and an overview of the current literature on exercise, particularly repeated bouts of exercise, is provided in Chapter 4. In addition, this chapter describes findings of a small-scale pilot study, in which we studied whether repetitive strenuous cycling had an additive, exhaustive, or adaptive effect on procoagulant and anticoagulant changes in 5 healthy male volunteers. Chapter $\mathbf{5}$ describes another cycling study, but this time hypoxia is introduced as a variable by studying the effects of repeated exercise on coagulation, amongst others VWF, at sea level versus $3,375 \mathrm{~m}$ altitude. 
After examining active VWF in healthy individuals in the first two parts, we describe studies in three patient populations in Part III of this thesis. Chapter $\mathbf{6}$ describes a prospective cohort study assessing the effects of an exacerbation of COPD on VWF parameters (as markers of endothelial activation), platelet activation and plasmatic coagulation. We specifically looked into the association of each of these components of coagulation with inflammation in these patients, who are known to have a strongly increased risk of a cardiovascular event during and shortly following an exacerbation.

Another condition associated with both increased inflammation and high thrombotic risk is chronic kidney disease (CKD). For the study described in Chapter 7, we determined active VWF levels in plasma from a cohort of patients with CKD or end-stage renal disease (ESRD), receiving haemodialysis or peritoneal dialysis. We assessed whether levels of active VWF (and VWF:Ag) are differentially affected by various stages of kidney disease and dialysis modalities.

While most studies on active VWF focus on pathologies associated with thrombotic complications due to increased active VWF levels, spontaneous interaction between active VWF and platelets can also tilt the haemostatic balance to the bleeding side, as exemplified by VWD type 2B. However, it is unknown whether the proportion of activated VWF in the circulation also plays a role in patients with mild to moderate bleeding without thrombocytopenia. Therefore, in Chapter 8, we investigated whether in a population of patients with a mild bleeding diathesis more patients had very low active VWF levels compared to the control population. In addition, we sought to identify cases in which active VWF levels may have additional diagnostic value over the commonly used VWF:Ag and VWF:RCo assays.

The haemostatic potential of VWF largely depends on its multimer size and is therefore primarily regulated by ADAMTS13-mediated proteolysis. The conformation of VWF is important for this process, as ADAMTS13 can only cleave the A2 domain of VWF in its unfolded, active conformation. Over the past years, there has been increasing attention for plasmin as a potential back-up for ADAMTS13 in proteolysis of UL-VWF, in particular in conditions with reduced ADAMTS13 activity, such as TTP. ${ }^{152,153}$ Plasmin has a limited affinity for globular VWF, but its affinity is strongly enhanced upon activation of VWF. In Chapter 9, we review the existing literature on the effects of plasmin on VWF multimeric structure, VWF binding to platelets, and on platelet activation. This information is placed in the context of possible applications of thrombolytic therapy for TTP.

To conclude, the most important findings of this thesis, implications for clinical (laboratory) practice and future research directions are discussed in Chapter 10. This is followed by a summary in Dutch in Chapter 11. 


\section{REFERENCES}

1. Doolittle RF. The evolution of vertebrate blood coagulation: a case of Yin and Yang. Thromb Haemost. 1993;70(1):24-8.

2. Versteeg HH, Heemskerk JW, Levi M, Reitsma PH. New fundamentals in hemostasis. Physiol Rev. 2013;93(1):327-58.

3. Dahlback B. Blood coagulation. Lancet. 2000;355(9215):1627-32.

4. Furie B, Furie BC. Mechanisms of thrombus formation. N Engl J Med. 2008;359(9):938-49.

5. Yau JW, Teoh H, Verma S. Endothelial cell control of thrombosis. BMC Cardiovasc Disord. 2015;15:130.

6. Hoffman M. A cell-based model of coagulation and the role of factor VIla. Blood Rev. 2003;17 Suppl 1:S1-5.

7. Monroe DM, Hoffman M. What does it take to make the perfect clot? Arterioscler Thromb Vasc Biol. 2006;26(1):41-8.

8. Broos K, Feys HB, De Meyer SF, Vanhoorelbeke K, Deckmyn H. Platelets at work in primary hemostasis. Blood Rev. 2011;25(4):155-67.

9. Pugh N, Maddox BD, Bihan D, Taylor KA, Mahaut-Smith MP, Farndale RW. Differential integrin activity mediated by platelet collagen receptor engagement under flow conditions. Thromb Haemost. 2017;117(8):1588-600.

10. Munnix IC, Cosemans JM, Auger JM, Heemskerk JW. Platelet response heterogeneity in thrombus formation. Thromb Haemost. 2009;102(6):1149-56.

11. Bryckaert M, Rosa JP, Denis CV, Lenting PJ. Of von Willebrand factor and platelets. Cell Mol Life Sci. 2015;72(2):307-26.

12. Furlan $M$, Robles $R$, Lammle B. Partial purification and characterization of a protease from human plasma cleaving von Willebrand factor to fragments produced by in vivo proteolysis. Blood. 1996;87(10):4223-34.

13. Tsai HM. Physiologic cleavage of von Willebrand factor by a plasma protease is dependent on its conformation and requires calcium ion. Blood. 1996;87(10):4235-44.

14. Hartwig JH. The platelet: form and function. Semin Hematol. 2006;43(1 Suppl 1):S94-100.

15. Blair P, Flaumenhaft R. Platelet alpha-granules: basic biology and clinical correlates. Blood Rev. 2009;23(4):177-89.

16. Duvernay MT, Temple KJ, Maeng JG, Blobaum AL, Stauffer SR, Lindsley CW, et al. Contributions of Protease-Activated Receptors PAR1 and PAR4 to Thrombin-Induced GPIIbllla Activation in Human Platelets. Mol Pharmacol. 2017;91(1):39-47.

17. Remijn JA, Wu YP, Jeninga EH, MJ IJ, van Willigen G, de Groot PG, et al. Role of ADP receptor $\mathrm{P} 2 \mathrm{Y}(12)$ in platelet adhesion and thrombus formation in flowing blood. Arterioscler Thromb Vasc Biol. 2002;22(4):686-91.

18. Gralnick HR, Williams SB, Coller BS. Fibrinogen competes with von Willebrand factor for binding to the glycoprotein Ilb/Illa complex when platelets are stimulated with thrombin. Blood. 1984;64(4):797-800.

19. French DL, Seligsohn U. Platelet glycoprotein Ilb/Illa receptors and Glanzmann's thrombasthenia. Arterioscler Thromb Vasc Biol. 2000;20(3):607-10.

20. Sodergren AL, Ramstrom S. Platelet subpopulations remain despite strong dual agonist stimulation and can be characterised using a novel six-colour flow cytometry protocol. Sci Rep. 2018;8(1):1441. 
21. Monroe DM, Hoffman M, Roberts HR. Platelets and thrombin generation. Arterioscler Thromb Vasc Biol. 2002;22(9):1381-9.

22. Kalafatis M, Rand MD, Mann KG. The mechanism of inactivation of human factor $V$ and human factor Va by activated protein C. J Biol Chem. 1994;269(50):31869-80.

23. Suzuki K, Kusumoto H, Deyashiki Y, Nishioka J, Maruyama I, Zushi M, et al. Structure and expression of human thrombomodulin, a thrombin receptor on endothelium acting as a cofactor for protein C activation. EMBO J. 1987;6(7):1891-7.

24. Koutsi A, Papapanagiotou A, Papavassiliou AG. Thrombomodulin: from haemostasis to inflammation and tumourigenesis. Int J Biochem Cell Biol. 2008;40(9):1669-73.

25. Girard TJ, Warren LA, Novotny WF, Likert KM, Brown SG, Miletich JP, et al. Functional significance of the Kunitz-type inhibitory domains of lipoprotein-associated coagulation inhibitor. Nature. 1989;338(6215):518-20.

26. Castoldi E, Simioni P, Tormene D, Rosing J, Hackeng TM. Hereditary and acquired protein S deficiencies are associated with low TFPI levels in plasma. J Thromb Haemost. 2010;8(2):294300.

27. Cesarman-Maus G, Hajjar KA. Molecular mechanisms of fibrinolysis. Br J Haematol. 2005;129(3):307-21.

28. Thorsen S, Clemmensen I, Sottrup-Jensen L, Magnusson S. Adsorption to fibrin of native fragments of known primary structure from human plasminogen. Biochim Biophys Acta. 1981;668(3):377-87.

29. Medved L, Nieuwenhuizen W. Molecular mechanisms of initiation of fibrinolysis by fibrin. Thromb Haemost. 2003;89(3):409-19.

30. Samis JA, Ramsey GD, Walker JB, Nesheim ME, Giles AR. Proteolytic processing of human coagulation factor IX by plasmin. Blood. 2000;95(3):943-51.

31. Omar MN, Mann KG. Inactivation of factor Va by plasmin. J Biol Chem. 1987;262(20):97505.

32. Li A, Wun TC. Proteolysis of tissue factor pathway inhibitor (TFPI) by plasmin: effect on TFPI activity. Thromb Haemost. 1998;80(3):423-7.

33. Crawley JT, Lam JK, Rance JB, Mollica LR, O'Donnell JS, Lane DA. Proteolytic inactivation of ADAMTS13 by thrombin and plasmin. Blood. 2005;105(3):1085-93.

34. Holvoet $P$, Lijnen HR, Collen D. A monoclonal antibody specific for Lys-plasminogen. Application to the study of the activation pathways of plasminogen in vivo. J Biol Chem. 1985;260(22):12106-11.

35. Ny T, Sawdey M, Lawrence D, Millan JL, Loskutoff DJ. Cloning and sequence of a cDNA coding for the human beta-migrating endothelial-cell-type plasminogen activator inhibitor. Proc Natl Acad Sci U S A. 1986;83(18):6776-80.

36. Aoki N, Moroi M, Tachiya K. Effects of alpha2-plasmin inhibitor on fibrin clot lysis. Its comparison with alpha2-macroglobulin. Thromb Haemost. 1978;39(1):22-31.

37. Plug T, Meijers JC. Structure-function relationships in thrombin-activatable fibrinolysis inhibitor. J Thromb Haemost. 2016;14(4):633-44.

38. Kumar DR, Hanlin E, Glurich I, Mazza JJ, Yale SH. Virchow's contribution to the understanding of thrombosis and cellular biology. Clin Med Res. 2010;8(3-4):168-72.

39. Lip GY, Blann AD. Thrombogenesis and fibrinolysis in acute coronary syndromes. Important facets of a prothrombotic or hypercoagulable state? J Am Coll Cardiol. 2000;36(7):2044-6.

40. Diamond SL. Systems Analysis of Thrombus Formation. Circ Res. 2016;118(9):1348-62. 
41. Wolberg AS, Aleman MM, Leiderman K, Machlus KR. Procoagulant activity in hemostasis and thrombosis: Virchow's triad revisited. Anesth Analg. 2012;114(2):275-85.

42. Tan KT, Lip GY. Red vs white thrombi: treating the right clot is crucial. Arch Intern Med. 2003;163(20):2534-5; author reply 5.

43. Triplett DA. Coagulation and bleeding disorders: review and update. Clin Chem. 2000;46(8 Pt 2):1260-9.

44. Peyvandi F, Garagiola I, Young G. The past and future of haemophilia: diagnosis, treatments, and its complications. Lancet. 2016;388(10040):187-97.

45. Nurden AT, Nurden P. Inherited disorders of platelet function: selected updates. J Thromb Haemost. 2015;13 Suppl 1:S2-9.

46. Rodeghiero F, Castaman G, Dini E. Epidemiological investigation of the prevalence of von Willebrand's disease. Blood. 1987;69(2):454-9.

47. Castaman G, Linari S. Diagnosis and Treatment of von Willebrand Disease and Rare Bleeding Disorders. J Clin Med. 2017;6(4).

48. Sadler JE. New concepts in von Willebrand disease. Annu Rev Med. 2005;56:173-91.

49. Jenkins PV, Rawley O, Smith OP, O'Donnell JS. Elevated factor VIII levels and risk of venous thrombosis. Br J Haematol. 2012;157(6):653-63.

50. Verweij CL, Diergaarde PJ, Hart M, Pannekoek H. Full-length von Willebrand factor (vWF) CDNA encodes a highly repetitive protein considerably larger than the mature vWF subunit. EMBO J. 1986;5(8):1839-47.

51. Kanaji S, Fahs SA, Shi O, Haberichter SL, Montgomery RR. Contribution of platelet vs. endothelial VWF to platelet adhesion and hemostasis. J Thromb Haemost. 2012;10(8):1646-52.

52. Zhou YF, Eng ET, Zhu J, Lu C, Walz T, Springer TA. Sequence and structure relationships within von Willebrand factor. Blood. 2012;120(2):449-58.

53. van der Vorm LN, Li L, Huskens D, Chayoua W, Kelchtermans H, de Groot PG, et al. Analytical characterization and reference interval of an enzyme-linked immunosorbent assay for active von Willebrand factor. PLoS One. 2019;14(2):e0211961.

54. Purvis AR, Gross J, Dang LT, Huang RH, Kapadia M, Townsend RR, et al. Two Cys residues essential for von Willebrand factor multimer assembly in the Golgi. Proc Natl Acad Sci U S A. 2007;104(40):15647-52.

55. Foster PA, Fulcher CA, Marti T, Titani K, Zimmerman TS. A major factor VIII binding domain resides within the amino-terminal 272 amino acid residues of von Willebrand factor. J Biol Chem. 1987;262(18):8443-6.

56. Huizinga EG, Tsuji S, Romijn RA, Schiphorst ME, de Groot PG, Sixma J], et at. Structures of glycoprotein Ibalpha and its complex with von Willebrand factor A1 domain. Science. 2002;297(5584):1176-9.

57. Hoylaerts MF, Yamamoto H, Nuyts K, Vreys I, Deckmyn H, Vermylen J. von Willebrand factor binds to native collagen VI primarily via its A1 domain. Biochem J. 1997;324 ( Pt 1):185-91.

58. Mohri H, Yoshioka A, Zimmerman TS, Ruggeri ZM. Isolation of the von Willebrand factor domain interacting with platelet glycoprotein $\mathrm{lb}$, heparin, and collagen and characterization of its three distinct functional sites. J Biol Chem. 1989;264(29):17361-7.

59. Fujikawa K, Suzuki H, McMullen B, Chung D. Purification of human von Willebrand factorcleaving protease and its identification as a new member of the metalloproteinase family. Blood. 2001;98(6):1662-6. 
60. Wu JJ, Fujikawa K, McMullen BA, Chung DW. Characterization of a core binding site for ADAMTS-13 in the A2 domain of von Willebrand factor. Proc Natl Acad Sci U S A. 2006;103(49):18470-4.

61. Romijn RA, Westein E, Bouma B, Schiphorst ME, Sixma JJ, Lenting PJ, et al. Mapping the collagen-binding site in the von Willebrand factor-A3 domain. J Biol Chem. 2003;278(17):150359.

62. Katsumi A, Tuley EA, Bodo I, Sadler JE. Localization of disulfide bonds in the cystine knot domain of human von Willebrand factor. J Biol Chem. 2000;275(33):25585-94.

63. Haberichter SL, Fahs SA, Montgomery RR. von Willebrand factor storage and multimerization: 2 independent intracellular processes. Blood. 2000;96(5):1808-15.

64. Sadler JE. Biochemistry and genetics of von Willebrand factor. Annu Rev Biochem. 1998;67:395-424.

65. Vischer UM, Wagner DD. von Willebrand factor proteolytic processing and multimerization precede the formation of Weibel-Palade bodies. Blood. 1994;83(12):3536-44.

66. Wagner DD, Olmsted JB, Marder VJ. Immunolocalization of von Willebrand protein in Weibel-Palade bodies of human endothelial cells. J Cell Biol. 1982;95(1):355-60.

67. Andre P, Denis CV, Ware J, Saffaripour S, Hynes RO, Ruggeri ZM, et al. Platelets adhere to and translocate on von Willebrand factor presented by endothelium in stimulated veins. Blood. 2000;96(10):3322-8.

68. Nightingale T, Cutler D. The secretion of von Willebrand factor from endothelial cells; an increasingly complicated story. J Thromb Haemost. 2013;11 Suppl 1:192-201.

69. Haberichter SL. von Willebrand factor propeptide: biology and clinical utility. Blood. 2015;126(15):1753-61.

70. Hamilton KK, Fretto LJ, Grierson DS, McKee PA. Effects of plasmin on von Willebrand factor multimers. Degradation in vitro and stimulation of release in vivo. J Clin Invest. 1985;76(1):261-70.

71. van der Vorm LN, Remijn JA, De Laat B, Huskens D. Effects of Plasmin on von Willebrand Factor and Platelets: A Narrative Review. TH Open. 2018;2(2):e218-e28.

72. Haberichter SL, Merricks EP, Fahs SA, Christopherson PA, Nichols TC, Montgomery RR. Reestablishment of VWF-dependent Weibel-Palade bodies in VWD endothelial cells. Blood. 2005;105(1):145-52.

73. Brown SA, Eldridge A, Collins PW, Bowen DJ. Increased clearance of von Willebrand factor antigen post-DDAVP in Type 1 von Willebrand disease: is it a potential pathogenic process? J Thromb Haemost. 2003;1(8):1714-7.

74. Matsui T, Titani K, Mizuochi T. Structures of the asparagine-linked oligosaccharide chains of human von Willebrand factor. Occurrence of blood group $A, B$, and $H(O)$ structures. J Biol Chem. 1992;267(13):8723-31.

75. Canis K, McKinnon TA, Nowak A, Haslam SM, Panico M, Morris HR, et al. Mapping the Nglycome of human von Willebrand factor. Biochem J. 2012;447(2):217-28.

76. Gallinaro L, Cattini MG, Sztukowska M, Padrini R, Sartorello F, Pontara E, et al. A shorter von Willebrand factor survival in $O$ blood group subjects explains how $A B O$ determinants influence plasma von Willebrand factor. Blood. 2008;111(7):3540-5.

77. Jenkins PV, O'Donnell JS. ABO blood group determines plasma von Willebrand factor levels: a biologic function after all? Transfusion. 2006;46(10):1836-44. 
78. Canis K, McKinnon TA, Nowak A, Panico M, Morris HR, Laffan M, et al. The plasma von Willebrand factor O-glycome comprises a surprising variety of structures including $A B H$ antigens and disialosyl motifs. J Thromb Haemost. 2010;8(1):137-45.

79. McGrath RT, van den Biggelaar M, Byrne B, O'Sullivan JM, Rawley O, O'Kennedy R, et al. Altered glycosylation of platelet-derived von Willebrand factor confers resistance to $A D$ AMTS13 proteolysis. Blood. 2013;122(25):4107-10.

80. van Schooten CJ, Shahbazi S, Groot E, Oortwijn BD, van den Berg HM, Denis CV, et al. Macrophages contribute to the cellular uptake of von Willebrand factor and factor VIII in vivo. Blood. 2008;112(5):1704-12.

81. Casari C, Lenting PJ, Wohner N, Christophe OD, Denis CV. Clearance of von Willebrand factor. J Thromb Haemost. 2013;11 Suppl 1:202-11.

82. Grewal PK, Uchiyama S, Ditto D, Varki N, Le DT, Nizet V, et al. The Ashwell receptor mitigates the lethal coagulopathy of sepsis. Nat Med. 2008;14(6):648-55.

83. van Schooten CJ, Denis CV, Lisman T, Eikenboom JC, Leebeek FW, Goudemand J, et al. Variations in glycosylation of von Willebrand factor with 0-linked sialylated T antigen are associated with its plasma levels. Blood. 2007;109(6):2430-7.

84. Ward SE, O'Sullivan JM, Drakeford C, Aguila S, Jondle CN, Sharma J, et al. A novel role for the macrophage galactose-type lectin receptor in mediating von Willebrand factor clearance. Blood. 2018;131(8):911-6.

85. Pegon JN, Kurdi M, Casari C, Odouard S, Denis CV, Christophe OD, et al. Factor VIII and von Willebrand factor are ligands for the carbohydrate-receptor Siglec-5. Haematologica. 2012;97(12):1855-63.

86. Rastegarlari G, Pegon JN, Casari C, Odouard S, Navarrete AM, Saint-Lu N, et al. Macrophage LRP1 contributes to the clearance of von Willebrand factor. Blood. 2012;119(9):2126-34.

87. Lenting PJ, Neels JG, van den Berg BM, Clijsters PP, Meijerman DW, Pannekoek H, et al. The light chain of factor VIII comprises a binding site for low density lipoprotein receptorrelated protein. J Biol Chem. 1999;274(34):23734-9.

88. Castro-Nunez L, Dienava-Verdoold I, Herczenik E, Mertens K, Meijer AB. Shear stress is required for the endocytic uptake of the factor VIII-von Willebrand factor complex by macrophages. J Thromb Haemost. 2012;10(9):1929-37.

89. Rydz N, Swystun LL, Notley C, Paterson AD, Riches J], Sponagle K, et al. The C-type lectin receptor CLEC4M binds, internalizes, and clears von Willebrand factor and contributes to the variation in plasma von Willebrand factor levels. Blood. 2013;121(26):5228-37.

90. Ruggeri ZM. The role of von Willebrand factor in thrombus formation. Thromb Res. 2007;120 Suppl 1:S5-9.

91. Moroi M, Jung SM. A mechanism to safeguard platelet adhesion under high shear flow: von Willebrand factor-glycoprotein $\mathrm{lb}$ and integrin alphabeta-collagen interactions make complementary, collagen-type-specific contributions to adhesion. J Thromb Haemost. 2007:5(4):797-803.

92. Lenting PJ, CJ VANS, Denis CV. Clearance mechanisms of von Willebrand factor and factor VIII. J Thromb Haemost. 2007;5(7):1353-60.

93. Terraube V, O'Donnell JS, Jenkins PV. Factor VIII and von Willebrand factor interaction: biological, clinical and therapeutic importance. Haemophilia. 2010;16(1):3-13.

94. Slayter H, Loscalzo J, Bockenstedt P, Handin RI. Native conformation of human von Willebrand protein. Analysis by electron microscopy and quasi-elastic light scattering. J Biol Chem. 1985;260(14):8559-63. 
95. Tischer A, Machha VR, Moon-Tasson L, Benson LM, Auton M. Glycosylation Sterically Inhibits Platelet Adhesion to von Willebrand Factor without Altering Intrinsic Conformational Dynamics. J Thromb Haemost. 2019.

96. Deng W, Wang Y, Druzak SA, Healey JF, Syed AK, Lollar P, et al. A discontinuous autoinhibitory module masks the A1 domain of von Willebrand factor. J Thromb Haemost. 2017;15(9):186777.

97. Schneider SW, Nuschele S, Wixforth A, Gorzelanny C, Alexander-Katz A, Netz RR, et al. Shearinduced unfolding triggers adhesion of von Willebrand factor fibers. Proc Natl Acad Sci U S A. 2007;104(19):7899-903.

98. Ruggeri ZM, Orje JN, Habermann R, Federici AB, Reininger AJ. Activation-independent platelet adhesion and aggregation under elevated shear stress. Blood. 2006;108(6):1903-10.

99. Savage B, Sixma J], Ruggeri ZM. Functional self-association of von Willebrand factor during platelet adhesion under flow. Proc Natl Acad Sci U S A. 2002;99(1):425-30.

100. Hoeks AP, Samijo SK, Brands PJ, Reneman RS. Noninvasive determination of shear-rate distribution across the arterial lumen. Hypertension. 1995;26(1):26-33.

101. Chen J, Ling M, Fu X, Lopez JA, Chung DW. Simultaneous exposure of sites in von Willebrand factor for glycoprotein lb binding and ADAMTS13 cleavage: studies with ristocetin. Arterioscler Thromb Vasc Biol. 2012;32(11):2625-30.

102. Arya M, Anvari B, Romo GM, Cruz MA, Dong JF, McIntire LV, et al. Ultralarge multimers of von Willebrand factor form spontaneous high-strength bonds with the platelet glycoprotein Ib-IX complex: studies using optical tweezers. Blood. 2002;99(11):3971-7.

103. Furlan M. Von Willebrand factor: molecular size and functional activity. Ann Hematol. 1996;72(6):341-8.

104. Zheng X, Chung D, Takayama TK, Majerus EM, Sadler JE, Fujikawa K. Structure of von Willebrand factor-cleaving protease (ADAMTS13), a metalloprotease involved in thrombotic thrombocytopenic purpura. J Biol Chem. 2001;276(44):41059-63.

105. Zhang O, Zhou YF, Zhang CZ, Zhang X, Lu C, Springer TA. Structural specializations of A2, a force-sensing domain in the ultralarge vascular protein von Willebrand factor. Proc Natl Acad Sci U S A. 2009;106(23):9226-31.

106. Crawley JT, de Groot R, Xiang Y, Luken BM, Lane DA. Unraveling the scissile bond: how ADAMTS13 recognizes and cleaves von Willebrand factor. Blood. 2011;118(12):3212-21.

107. Martin C, Morales LD, Cruz MA. Purified A2 domain of von Willebrand factor binds to the active conformation of von Willebrand factor and blocks the interaction with platelet glycoprotein Ibalpha. J Thromb Haemost. 2007;5(7):1363-70.

108. Hulstein JJ, de Groot PG, Silence K, Veyradier A, Fijnheer R, Lenting PJ. A novel nanobody that detects the gain-of-function phenotype of von Willebrand factor in ADAMTS13 deficiency and von Willebrand disease type 2B. Blood. 2005;106(9):3035-42.

109. Groot E, de Groot PG, Fijnheer R, Lenting PJ. The presence of active von Willebrand factor under various pathological conditions. Curr Opin Hematol. 2007;14(3):284-9.

110. Ruggeri ZM. Type IIB von Willebrand disease: a paradox explains how von Willebrand factor works. J Thromb Haemost. 2004;2(1):2-6.

111. Sadler JE. A revised classification of von Willebrand disease. For the Subcommittee on von Willebrand Factor of the Scientific and Standardization Committee of the International Society on Thrombosis and Haemostasis. Thromb Haemost. 1994;71(4):520-5. 
112. Sadler JE, Budde U, Eikenboom JC, Favaloro EJ, Hill FG, Holmberg L, et al. Update on the pathophysiology and classification of von Willebrand disease: a report of the Subcommittee on von Willebrand Factor. J Thromb Haemost. 2006;4(10):2103-14.

113. Cooney KA, Nichols WC, Bruck ME, Bahou WF, Shapiro AD, Bowie EJ, et al. The molecular defect in type IIB von Willebrand disease. Identification of four potential missense mutations within the putative Gplb binding domain. J Clin Invest. 1991;87(4):1227-33.

114. Randi AM, Rabinowitz I, Mancuso DJ, Mannucci PM, Sadler JE. Molecular basis of von Willebrand disease type IIB. Candidate mutations cluster in one disulfide loop between proposed platelet glycoprotein Ib binding sequences. J Clin Invest. 1991;87(4):1220-6.

115. De Marco L, Girolami A, Zimmerman TS, Ruggeri ZM. Interaction of purified type IIB von Willebrand factor with the platelet membrane glycoprotein $\mathrm{lb}$ induces fibrinogen binding to the glycoprotein Ilb/Illa complex and initiates aggregation. Proc Natl Acad Sci U S A. 1985;82(21):7424-8.

116. Rayes J, Hollestelle MJ, Legendre P, Marx I, de Groot PG, Christophe OD, et al. Mutation and ADAMTS13-dependent modulation of disease severity in a mouse model for von Willebrand disease type 2B. Blood. 2010;115(23):4870-7.

117. Casonato A, Daidone V, Galletta E, Bertomoro A. Type 2B von Willebrand disease with or without large multimers: A distinction of the two sides of the disorder is long overdue. PLoS One. 2017;12(6):e0179566.

118. Federici AB, Mannucci PM, Castaman G, Baronciani L, Bucciarelli P, Canciani MT, et al. Clinical and molecular predictors of thrombocytopenia and risk of bleeding in patients with von Willebrand disease type 2B: a cohort study of 67 patients. Blood. 2009;113(3):526-34.

119. Peyvandi F, Hollestelle MJ, Palla R, Merlini PA, Feys HB, Vanhoorelbeke K, et al. Active platelet-binding conformation of plasma von Willebrand factor in young women with acute myocardial infarction. J Thromb Haemost. 2010;8(7):1653-6.

120. Rutten B, Maseri A, Cianflone D, Laricchia A, Cristell NA, Durante A, et al. Plasma levels of active Von Willebrand factor are increased in patients with first ST-segment elevation myocardial infarction: a multicenter and multiethnic study. Eur Heart J Acute Cardiovasc Care. 2015;4(1):64-74.

121. Sadler JE. Pathophysiology of thrombotic thrombocytopenic purpura. Blood. 2017;130(10):1181-8.

122. Scully M, Yarranton H, Liesner R, Cavenagh J, Hunt B, Benjamin S, et al. Regional UK TTP registry: correlation with laboratory ADAMTS 13 analysis and clinical features. $\mathrm{Br}$ J Haematol. 2008;142(5):819-26.

123. Cataland SR, Wu HM. Atypical hemolytic uremic syndrome and thrombotic thrombocytopenic purpura: clinically differentiating the thrombotic microangiopathies. Eur J Intern Med. 2013;24(6):486-91.

124. Levy GG, Nichols WC, Lian EC, Foroud T, McClintick JN, McGee BM, et al. Mutations in a member of the ADAMTS gene family cause thrombotic thrombocytopenic purpura. Nature. 2001;413(6855):488-94.

125. Moake JL. Thrombotic microangiopathies. N Engl J Med. 2002;347(8):589-600.

126. Dusse LM, Alpoim PN, Silva JT, Rios DR, Brandao AH, Cabral AC. Revisiting HELLP syndrome. Clin Chim Acta. 2015;451(Pt B):117-20.

127. Hulstein JJ, van Runnard Heimel PJ, Franx A, Lenting PJ, Bruinse HW, Silence K, et al. Acute activation of the endothelium results in increased levels of active von Willebrand factor in 
hemolysis, elevated liver enzymes and low platelets (HELLP) syndrome. J Thromb Haemost. 2006;4(12):2569-75.

128. Lattuada A, Rossi E, Calzarossa C, Candolfi R, Mannucci PM. Mild to moderate reduction of a von Willebrand factor cleaving protease (ADAMTS-13) in pregnant women with HELLP microangiopathic syndrome. Haematologica. 2003;88(9):1029-34.

129. de Mast Q, Groot E, Lenting PJ, de Groot PG, McCall M, Sauerwein RW, et al. Thrombocytopenia and release of activated von Willebrand Factor during early Plasmodium falciparum malaria. J Infect Dis. 2007;196(4):622-8.

130. de Mast O, Groot E, Asih PB, Syafruddin D, Oosting M, Sebastian S, et al. ADAMTS13 deficiency with elevated levels of ultra-large and active von Willebrand factor in P. falciparum and P. vivax malaria. Am J Trop Med Hyg. 2009;80(3):492-8.

131. Djamiatun K, van der Ven AJ, de Groot PG, Faradz SM, Hapsari D, Dolmans WM, et al. Severe dengue is associated with consumption of von Willebrand factor and its cleaving enzyme ADAMTS-13. PLoS Negl Trop Dis. 2012;6(5):e1628.

132. Riswari SF, Tunjungputri RN, Kullaya V, Garishah FM, Utari GSR, Farhanah N, et al. Desialylation of platelets induced by Von Willebrand Factor is a novel mechanism of platelet clearance in dengue. PLoS Pathog. 2019;15(3):e1007500.

133. Pober JS, Sessa WC. Evolving functions of endothelial cells in inflammation. Nat Rev Immunol. 2007;7(10):803-15.

134. van Mourik JA, Boertjes R, Huisveld IA, Fijnvandraat K, Pajkrt D, van Genderen PJ, et al. von Willebrand factor propeptide in vascular disorders: $A$ tool to distinguish between acute and chronic endothelial cell perturbation. Blood. 1999;94(1):179-85.

135. Borchiellini A, Fijnvandraat K, ten Cate JW, Pajkrt D, van Deventer SJ, Pasterkamp G, et al. Quantitative analysis of von Willebrand factor propeptide release in vivo: effect of experimental endotoxemia and administration of 1-deamino-8-D-arginine vasopressin in humans. Blood. 1996;88(8):2951-8.

136. Bockmeyer CL, Claus RA, Budde U, Kentouche K, Schneppenheim R, Losche W, et al. Inflammation-associated ADAMTS13 deficiency promotes formation of ultra-large von Willebrand factor. Haematologica. 2008;93(1):137-40.

137. Ono T, Mimuro J, Madoiwa S, Soejima K, Kashiwakura Y, Ishiwata A, et al. Severe secondary deficiency of von Willebrand factor-cleaving protease (ADAMTS13) in patients with sepsisinduced disseminated intravascular coagulation: its correlation with development of renal failure. Blood. 2006;107(2):528-34.

138. Reiter RA, Varadi K, Turecek PL, Jilma B, Knobl P. Changes in ADAMTS13 (von-Willebrandfactor-cleaving protease) activity after induced release of von Willebrand factor during acute systemic inflammation. Thromb Haemost. 2005;93(3):554-8.

139. Bernardo A, Ball C, Nolasco L, Moake JF, Dong JF. Effects of inflammatory cytokines on the release and cleavage of the endothelial cell-derived ultralarge von Willebrand factor multimers under flow. Blood. 2004;104(1):100-6.

140. Kawecki C, Lenting PJ, Denis CV. von Willebrand factor and inflammation. J Thromb Haemost. 2017;15(7):1285-94.

141. Pendu R, Terraube V, Christophe OD, Gahmberg CG, de Groot PG, Lenting PJ, et al. P-selectin glycoprotein ligand 1 and beta2-integrins cooperate in the adhesion of leukocytes to von Willebrand factor. Blood. 2006;108(12):3746-52.

142. Petri B, Broermann A, Li H, Khandoga AG, Zarbock A, Krombach F, et al. von Willebrand factor promotes leukocyte extravasation. Blood. 2010;116(22):4712-9. 
143. Denis CV, Andre P, Saffaripour S, Wagner DD. Defect in regulated secretion of P-selectin affects leukocyte recruitment in von Willebrand factor-deficient mice. Proc Natl Acad Sci U S A. 2001;98(7):4072-7.

144. Hillgruber C, Steingraber AK, Poppelmann B, Denis CV, Ware J, Vestweber D, et al. Blocking von Willebrand factor for treatment of cutaneous inflammation. J Invest Dermatol. 2014;134(1):77-86.

145. Zhu X, Cao Y, Wei L, Cai P, Xu H, Luo H, et al. von Willebrand factor contributes to poor outcome in a mouse model of intracerebral haemorrhage. Sci Rep. 2016;6:35901.

146. McEver RP. Adhesive interactions of leukocytes, platelets, and the vessel wall during hemostasis and inflammation. Thromb Haemost. 2001;86(3):746-56.

147. Simon DI, Chen Z, Xu H, Li CO, Dong J, Mclntire LV, et al. Platelet glycoprotein ibalpha is a counterreceptor for the leukocyte integrin Mac-1 (CD11b/CD18). J Exp Med. 2000;192(2):193204.

148. Adam F, Casari C, Prevost N, Kauskot A, Loubiere C, Legendre P, et al. A genetically-engineered von Willebrand disease type $2 \mathrm{~B}$ mouse model displays defects in hemostasis and inflammation. Sci Rep. 2016;6:26306.

149. Hyseni A, Kemperman H, de Lange DW, Kesecioglu J, de Groot PG, Roest M. Active von Willebrand factor predicts 28-day mortality in patients with systemic inflammatory response syndrome. Blood. 2014;123(14):2153-6.

150. Scully M, Cataland SR, Peyvandi F, Coppo P, Knobl P, Kremer Hovinga JA, et al. Caplacizumab Treatment for Acquired Thrombotic Thrombocytopenic Purpura. N Engl J Med. 2019;380(4):335-46.

151. Callewaert F, Roodt J, Ulrichts H, Stohr T, van Rensburg WJ, Lamprecht $\mathrm{S}$, et al. Evaluation of efficacy and safety of the anti-VWF Nanobody ALX-0681 in a preclinical baboon model of acquired thrombotic thrombocytopenic purpura. Blood. 2012;120(17):3603-10.

152. Brophy TM, Ward SE, McGimsey TR, Schneppenheim S, Drakeford C, O'Sullivan JM, et al. Plasmin Cleaves Von Willebrand Factor at K1491-R1492 in the A1-A2 Linker Region in a Shearand Glycan-Dependent Manner In Vitro. Arterioscler Thromb Vasc Biol. 2017;37(5):845-55.

153. Tersteeg $C$, de Maat S, De Meyer SF, Smeets MW, Barendrecht AD, Roest M, et al. Plasmin cleavage of von Willebrand factor as an emergency bypass for ADAMTS13 deficiency in thrombotic microangiopathy. Circulation. 2014;129(12):1320-31. 


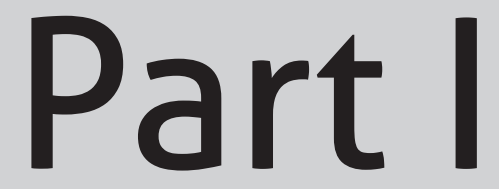


Detection of active VWF by a $\mathrm{VHH}$ : mechanisms and assay

\section{validation}




$$
\text { Chapter } 2
$$




\section{A variable heavy chain antibody against active von Willebrand factor only prevents platelet-VWF binding in solution}

Van der Vorm LN, Huskens D, Konings ], Roest M, de Groot PG, de Laat B, Remijn JA 


\section{ABSTRACT}

Background - Neutralizing the interaction of GP1b $\alpha$ with von Willebrand Factor (VWF) is an attractive strategy to prevent pathological platelet-VWF interaction. The variable heavy chain antibody (VHH) AU/VWFa-11, targeting the A1 domain of active VWF, was previously reported to not disturb platelet-VWF binding under flow. However, we observed inhibitory effects of a VHH (S-VWFA1), raised against the same epitope as AU/ VWFa-11, in other functional assays.

Aim - This study aimed to characterize the VHH S-VWFA1 in terms of its interference with platelet-VWF interaction.

Methods and Results - S-VWFA1 $(25 \mu \mathrm{g} / \mathrm{mL})$ abolished platelet binding to ristocetinactivated VWF and R1306W VWF in a flow-cytometric platelet function assay. Moreover, S-VWFA1 dose-dependently inhibited ristocetin- and R1306W VWF-, but not collagenand ADP-induced platelet aggregation in light transmission aggregometry. However, in a perfusion assay, S-VWFA1 $(30 \mu \mathrm{g} / \mathrm{mL})$ did not affect the adhesion of platelets to collagen under high shear $\left(1,600 \mathrm{~s}^{-1}\right)$. In the shear- and collagen-dependent PFA-200®, S-VWFA1 prolonged the closure time, but only at considerably higher concentrations (>50 $\mu \mathrm{g} / \mathrm{mL}$ ). GPIb $\alpha$ binding to collagen-immobilized VWF remained unaffected by SVWFA1.

Conclusions - S-VWFA1 dose-dependently blocks platelet-binding to activated VWF in solution, but not to collagen-immobilized active VWF. This implies that S-VWFA1 has the potential to prevent pathological platelet-VWF aggregate formation while leaving physiological haemostasis at sites of vascular injury unaffected. 


\section{INTRODUCTION}

Platelets and von Willebrand Factor (VWF) coexist in the circulation, however, complex formation does not occur spontaneously. Under physiological blood flow conditions, VWF multimers adopt a folded, globular conformation that shields the A1 domain from interaction with platelet glycoprotein (GP) $1 b \alpha$. Upon vascular injury, immobilization to subendothelial collagen and increased shear stress induce VWF unfolding. ${ }^{1}$ This conformational shift exposes the VWF A1 domain, allowing for platelet-VWF interaction. ${ }^{2}$

Premature and/or excessive VWF-platelet interaction occurs in a number of pathological conditions. ${ }^{3}$ In von Willebrand disease (VWD) type 2B, gain-of-function mutations (e.g. R1306W and R13060) in the A1 domain render VWF constitutively active, i.e. in a GPIb $\alpha$-binding conformation. ${ }^{4}$ The increased affinity for GP1b $\alpha$ results in the consumption of high-molecular-weight VWF multimers and consequently in thrombocytopenia and bleeding symptoms. ${ }^{5}$ Another condition associated with increased levels of active VWF is thrombotic thrombocytopenic purpura (TTP). ${ }^{6}$ In TTP patients, an acquired or inherited deficiency of the VWF-cleaving protease ADAMTS13 results in accumulation of ultra-large (UL)-VWF multimers ${ }^{7}$, mediating the spontaneous formation of plateletrich thrombi that occlude the microvasculature. ${ }^{8}$

Neutralizing the interaction of GP1b $\alpha$ with VWF is an attractive strategy to prevent pathological platelet-VWF interaction. Variable heavy chain antibody fragments (VHHs) against the VWF A1 domain have been raised previously. For instance, the bivalent VHH caplacizumab targets the A1 domain of both active and inactive (globular) VWF. ${ }^{9}$ Hulstein et al. previously reported on a recombinant VHH (AU/VWFa-11) produced in prokaryotic $E$. coli. AU/VWFa-11 only recognized the A1 domain of VWF in its activated state, ${ }^{6}$ which would be a benefit for potential therapeutic application. However, AU/ VWFa-11 could not inhibit platelet binding to collagen-bound VWF in a perfusion system $^{6}$ and was hence not developed further. We observed that a recombinant VHH (S-VWFA1) produced in eukaryotic HEK cells, targeting the same cryptic epitope in the A1-domain of $\mathrm{VWF}^{6}{ }^{6}$ did interfere with platelet-VWF binding in several assays other than the perfusion system. Therefore, we aimed to determine under what conditions this S-VWFA1 VHH modulates platelet-VWF interaction. 


\section{MATERIALS AND METHODS}

\section{Reagents}

Throughout all experiments, the VWF/FVIII concentrate Haemate ${ }^{\circledR}$ P VWF (CSL Behring, Pennsylvania, USA) (stock $12 \mathrm{mg} / \mathrm{mL}$ ) was used as native human VWF in its globular conformation, and is referred to as "HVWF". Recombinant VWF with the VWD type 2B R1306W (stock $17 \mu \mathrm{g} / \mathrm{mL}$ ) or R13060 (stock $55 \mu \mathrm{g} / \mathrm{mL}$ ) mutation (produced as described previously ${ }^{10}$ ) was used as VWF constitutively in the opened, active conformation. For the immunosorbent assay, antibodies raised against von Willebrand factor (A0082 and P0226) were purchased from Dako (Glostrup, Denmark). Ristocetin sulphate (100RIST001) was purchased from American Biochemical \& Pharmaceutical Ltd. (Marlton, USA) and bovine serum albumin (BSA; A7906), SIGMAFAST ${ }^{\mathrm{TM}}$ o-Phenylenediamine dihydrochloride (OPD; P9187) and sulfuric acid $\left(\mathrm{H}_{2} \mathrm{SO}_{4} ; 339741\right)$ were all purchased from Sigma-Aldrich (Zwijndrecht, The Netherlands). For the flow cytometric assay, FITCconjugated anti-VWF antibody (CL7616F) was purchased from Cedarlane Laboratories (Burlington, Canada) and PE-conjugated aCD41a (clone HIP8) from BD Biosciences (BD Pharmingen, Franklin Lakes, USA). Adenosine diphosphate (ADP; 01897) was purchased from Sigma-Aldrich (Zwijndrecht, the Netherlands), and Horm type I collagen (derived from equine tendon) from Nycomed (Munich, Germany). The 3,3' Dihexyloxacarbocyanine iodide (DiOC6; 8984715) used in the perfusion experiments was purchased from AnaSpec (Fremont, USA). GP1b $\alpha$ binding in the static assay was detected using recombinant human GP1b $\alpha$ (4067-GP), sheep anti-human GP1b $\alpha$ antibodies (AF4067) and HRP-conjugated donkey anti-sheep IgG antibodies (HAF016), all from R\&D Systems (Abingdon, UK).

\section{Production of the VHH S-VWFA1}

VWF A1 domain-specific VHH antibodies (MW $16.4 \mathrm{kDa}$ ) were produced by U-Protein Express BV (Utrecht, The Netherlands). In short, HEK293E-253 cells were transfected with endotoxin-free maxiprep DNA for the sequence of the S-VWFA1 VHH. Six days post-transfection conditioned medium containing recombinant protein was harvested by low-speed centrifugation (10 minutes, $1000 \mathrm{~g}$ ) followed by high-speed centrifugation (10 minutes, 4000g) and immobilized metal affinity chromatography (IMAC) purification. The S-VWFA1 VHH was further purified by gel filtration using a Superdex 75 $26 / 600$ column. The resulting $\mathrm{VHH}$-containing fractions were sterilized by filtration over a $0.22 \mu \mathrm{m}$ syringe filter and stored at $4^{\circ} \mathrm{C}$.

\section{Sample collection}

Blood was collected from healthy volunteers after full written informed consent, complying with the Declaration of Helsinki (version 2013). This study was approved 
by the Medical Research Ethics Committee of Maastricht University/University Hospital Maastricht, the Netherlands (METC azM/UM, reference NL67430.068.18). Donors did not take non-steroidal anti-inflammatory drugs (NSAIDs) for the preceding 3 days nor aspirin or clopidogrel for the preceding 10 days. Blood was collected by antecubital venipuncture into vacuum tubes ( 1 volume trisodium citrate $0.105 \mathrm{M}$ to 9 volumes blood) (BD Vacutainer System, Franklin Lakes, NJ, USA).

\section{Immunosorbent assay}

Overnight at $4^{\circ} \mathrm{C}, 96$ wells microtiter plates (Nunc Maxisorp, Sigma-Aldrich) were coated with $1.98 \mu \mathrm{g} / \mathrm{ml}$ S-VWFA1 VHH in $50 \mathrm{mM} \mathrm{NaHCO}$ coating buffer (pH 9.6). After blocking with $2 \%$ bovine serum albumin (BSA; Sigma-Aldrich) in phosphate-buffered saline (PBS) for 45 minutes at room temperature (RT), wells were washed with $0.1 \%$ Tween-20 in PBS. Normal pooled plasma (diluted 1:25, static or vortexed at 2,500 rpm for 10 minutes), HVWF, R1306W VWF, or R13060 VWF (all final concentration $1.7 \mu \mathrm{g} / \mathrm{mL}$ ), with or without ristocetin $(0.5 \mathrm{mg} / \mathrm{mL})$, were incubated for 2 hours at RT. After washing three times with $0.1 \%$ Tween-20 in PBS, wells were incubated with HRP-conjugated anti-VWF antibodies in PBS/1\%BSA for 2 hours at RT. Plates were washed three times before measuring VWF binding to S-VWFA1 by adding HRP substrate SIGMAFASTTM OPD. After 8 minutes, the reaction was stopped with $3 \mathrm{M}$ sulfuric acid $\left(\mathrm{H}_{2} \mathrm{SO}_{4}\right.$, Aldrich, USA). Optical densities at $490 \mathrm{~nm}$ were measured using an ELx808 Absorbance Microplate Reader (Biotek, USA).

\section{Flow cytometric analysis of platelet-VWF binding}

Binding of VWF to platelets was tested by adding $5 \mu \mathrm{L}$ of $1: 4$ diluted whole blood to 20 $\mu \mathrm{L}$ reaction mix consisting of $20 \mu \mathrm{g} / \mathrm{mL}$ HVWF or $4 \mu \mathrm{g} / \mathrm{mL}$ R1306W VWF (with or without $0.5 \mathrm{mg} / \mathrm{ml}$ ristocetin), $2 \mu \mathrm{l}$ FITC-conjugated anti-VWF antibody and $0.5 \mu \mathrm{LE}$-conjugated anti-CD 41a (directed against GPIIb and used as a platelet marker) in HEPES-buffered saline (HBS, $10 \mathrm{mmol} / \mathrm{L}$ HEPES, $150 \mathrm{mmol} / \mathrm{L} \mathrm{NaCl}, 1 \mathrm{mmol} / \mathrm{L} \mathrm{MgSO}_{4}, 5 \mathrm{mmol} / \mathrm{L} \mathrm{KCL}$, pH 7.4). The effect of S-VWFA1 on the binding of VWF to platelets was studied by adding $25 \mathrm{\mu g} / \mathrm{ml}$ S-VWFA1 to the reaction mix. Following incubation for $20 \mathrm{mins}$ at RT, the reactions were stopped by adding $250 \mu \mathrm{l}$ of fixation solution $(137 \mathrm{mmol} / \mathrm{L} \mathrm{NaCl}, 2.7 \mathrm{mmol} / \mathrm{L}$ $\mathrm{KCl}, 1.12 \mathrm{mmol} / \mathrm{L} \mathrm{NaH}_{2} \mathrm{PO}_{4}, 1.15 \mathrm{mmol} / \mathrm{L} \mathrm{KH}_{2} \mathrm{PO}_{4}, 10.2 \mathrm{mmol} / \mathrm{L} \mathrm{Na}{ }_{2} \mathrm{HPO}_{4}, 4 \mathrm{mmol} / \mathrm{L}$ EDTA, $0.5 \%$ formaldehyde). Flow cytometric analysis was performed on a BD Accuri flow cytometer (BD Pharmingen). A gate was placed on the CD41a positive cells to select for platelets. Binding of VWF to platelets was detected by the median fluorescent intensity (MFI) in the FL1 channel. 


\section{Light transmission aggregometry}

Platelet-rich plasma (PRP) and platelet-poor plasma (PPP) were prepared from citrated whole blood by centrifugation at $156 \mathrm{~g}$ for $15 \mathrm{~min}$ (PRP) and two times at $2500 \mathrm{~g}$ for $15 \mathrm{~min}$ (PPP). Platelet count in PRP was adjusted with autologous (PPP) to $250 \times 10^{9} / \mathrm{L}$. Light transmission aggregometry (LTA) was performed on a Chronolog aggregometer (Chrono-log Corporation, Havertown, PA, USA). Optimal platelet aggregation was defined as maximal light transmission obtained with autologous PPP. Increasing concentrations of the S-VWFA1 were added to pre-warmed $\left(37^{\circ} \mathrm{C}, 5 \mathrm{~min}\right)$ PRP and incubated for 20 minutes. Subsequently, platelet aggregation in PRP in response to ristocetin (final concentration $1.25 \mathrm{mg} / \mathrm{mL}$ ) was measured in the absence and presence of S-VWFA1 for 15 minutes. Similarly, platelet aggregation in PRP in response to ADP (final concentration $4 \mu \mathrm{M}$ ), collagen (final concentration $4 \mu \mathrm{g} / \mathrm{mL}$ ) and R1306W VWF (final concentration 3.4 $\mu \mathrm{g} / \mathrm{mL}$ ) in the absence and presence of $25 \mu \mathrm{g} / \mathrm{mL}$ of S-VWFA1 was assessed. Data were analysed using AGGRO/LINK software (Chrono-log). Aggregation was expressed as \% maximum aggregation compared to PPP, and the percentage (\%) inhibition of maximum platelet aggregation by the S-VWFA1 was calculated.

\section{Platelet adhesion to collagen and VWF under shear}

Microchannels of Vena8 Fluoro Biochip (Cellix Ltd, Dublin, Ireland) were coated with 12 $\mu \mathrm{L}$ of fibrillar Horm collagen type I at a concentration of $200 \mu \mathrm{g} / \mathrm{mL}$ for 2 hours at RT in a humidified box, blocked with $20 \mu \mathrm{L}$ of $10 \%$ BSA in HEPES (pH 7.45) for $15 \mathrm{~min}$ at RT, and washed with HEPES ( $\mathrm{pH} 7.45$ ) at $2000 \mathrm{~s}^{-1}$ for $1 \mathrm{~min}$. Citrate-anticoagulated whole blood was incubated with $30 \mu \mathrm{g} / \mathrm{mL} \mathrm{S}$-VWFA1 (or HEPES) and $\mathrm{DiCO}_{6}(1 \mu \mathrm{g} / \mathrm{ml})$ for $20 \mathrm{~min}$ at RT, protected from light. Blood was injected via a Kima microfluidic pump (Cellix Ltd.) into the microchannels at a fluid shear stress of $1600 \mathrm{~s}^{-1}$ within 4 hours of collection. After 4 min, non-bound platelets and other blood components were washed from the channels by infusing rinse buffer (HEPES $\mathrm{pH} 7.45,2 \mathrm{mM} \mathrm{CaCl}, 1 \mathrm{U} / \mathrm{mL}$ unfractionated heparin) for $4 \mathrm{~min}$ at $1600 \mathrm{~s}^{-1}$. Phase-contrast and fluorescent images (green fluorescent protein [GFP]) captured from 10 arbitrarily chosen microscopic fields per flow experiment were recorded with an EVOS microscope (Advanced Microscopy Group, Bothell, WA), equipped with a $60 \times$ oil ultra-transparent objective (Olympus, Hamburg, Germany), and accompanying software. The area covered with platelets (\% of the total image) and the density of platelets in aggregates were analysed with Image] software (www.imagej. net).

\section{Shear-dependent platelet function}

A Platelet Function Analyser 200 (INNOVANCE® PFA-200, Siemens Healthcare, Marburg, Germany) was used to measure the shear-dependent anti-VWF activity of S-VWFA1 in whole blood. S-VWFA1 (final concentrations between 12.5 and $100 \mu \mathrm{g} / \mathrm{mL}$ or PBS as a 
control) was added to citrated whole blood from three healthy volunteers. Following a 20 min incubation, $800 \mu \mathrm{L}$ of blood was added to a collagen/epinephrine or collagen/ ADP cartridge, according to the manufacturer's instructions. The closure time, i.e. the time in seconds to occlusion of the cartridge aperture, was recorded and results were expressed as \% inhibition of closure time relative to control (i.e. no S-VWFA1).

\section{Static binding of GP1b $\alpha$ to collagen-bound VWF}

To assess binding of GP1b $\alpha$ to collagen-bound VWF, HVWF was pre-incubated with ristocetin $(0.5 \mathrm{mg} / \mathrm{mL})$ in the absence or presence of S-VWFA1 VHH $(50 \mu \mathrm{g} / \mathrm{mL}$, " + VHH preinc"), and added to collagen type III coated wells of a commercial VWF-collagen binding (VWF:CB) assay (Asserachrom VWF:CB, 00239, Stago, Leiden, the Netherlands). After 2 hours of incubation at RT, wells were washed 3 times and subsequently incubated with saline (negative control) or solutions of human recombinant GP1b $\alpha(0.5 \mu \mathrm{g} / \mathrm{mL}$ ) with S-VWFA1 (50 $\mathrm{\mu g} / \mathrm{mL}$, " $+\mathrm{VHH}$ ") or saline ("-VHH" and "+VHH pre-inc") in dilution buffer (PBS/1\% BSA), again for 2 hours at RT. Following another wash step, binding of GP1b $\alpha$ was detected with sheep anti-human GP1b $\alpha$ antibodies $(2 \mu \mathrm{g} / \mathrm{mL})$ and HRP-conjugated donkey anti-sheep IgG antibodies (diluted 100-fold), both incubated 1.5 hours at RT with a washing step in between. OPD was used as a substrate, as described above for the active VWF immunosorbent assay.

\section{Statistical analysis}

All statistical analyses were performed using GraphPad Prism V5.0 for Windows (GraphPad Software, Inc. San Diego (A). All experiments were performed in triplicate unless stated otherwise, and all data are expressed as mean with SD. An unpaired t-test was performed to compare 2 conditions, and a one-way ANOVA followed by posthoc Bonferroni for the calculation of differences between more than 2 conditions. A P-value $<0.05$ was considered statistically significant.

\section{RESULTS}

\section{S-VWFA1 specifically recognizes VWF in a GPIb $\alpha$-binding conformation}

First, the specificity of S-VWFA1 for the active conformation of VWF was assessed by studying the binding of S-VWFA1 to HVWF, R1306W VWF, R13060 VWF, and VWF in plasma with an immunosorbent assay (Figure 1). S-VWFA1 could not interact with HVWF, however, when HVWF was preincubated with ristocetin, the signal increased 33-fold. Similarly, NPP incubated with S-VWFA1 resulted in a weak signal, while the signal increased in the presence of ristocetin. In contrast, VWF harbouring mutations 
in the A1 domain (R1306W or R13060), generating constitutively active VWF, showed spontaneous strong interaction with S-VWFA1. This interaction was further augmented by treatment with ristocetin. Finally, NPP was vortexed to simulate high shear stress. As a result, S-VWFA1 interacted with the shear-unfolded VWF in NPP. Treatment of shearunfolded VWF in NPP with ristocetin did not further increase binding to S-VWFA1.

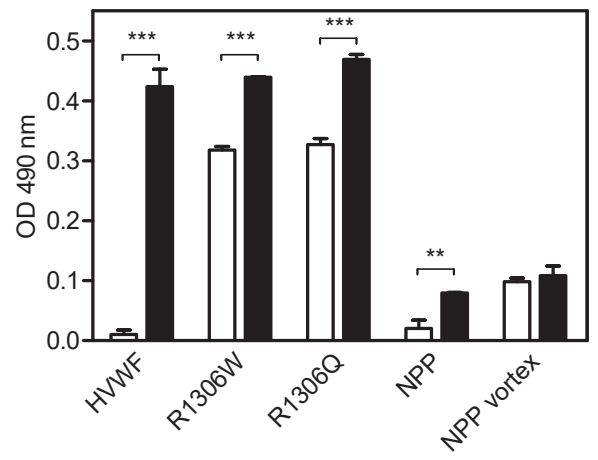

Figure 1. Interaction of S-VWFA1 with VWF in its native or activated conformation. The binding of S-VWFA1 to HVWF, R1306W, R13060, VWF in diluted NPP and VWF in vortexed diluted NPP treated with (black bars) or without (white bars) ristocetin. Data represent the mean $\pm S D(n=3) . * * P<0.001, * P<0.01$ (unpaired Student's t-test).

\section{S-VWFA1 inhibits ristocetin-induced platelet-VWF binding and aggregation}

Since activated VWF binds to Gplba on platelets, the next step was to investigate whether S-VWFA1 could inhibit this interaction in a whole blood flow cytometric assay. Native VWF and HVWF were only able to bind to platelets after pre-incubation with ristocetin (Figure 2A, B). R1306W VWF spontaneously bound to platelets and this binding was enhanced two-fold upon preincubation with ristocetin (Figure $2 \mathrm{C}$ ), corroborating our observations in the immunosorbent assay. Remarkably, S-VWFA1 abrogated both the spontaneous binding of R1306W VWF as well as the ristocetin-induced binding of native VWF, HVWF and R1306W VWF to platelets (Figure 2A-C).

The inhibitory effect of S-VWFA1 for ristocetin-induced VWF binding to platelets was confirmed with light transmission aggregometry (LTA) in platelet-rich plasma. S-VWFA1 dose-dependently inhibited ristocetin-induced platelet agglutination (Figure 2D), while ADP- and collagen-induced platelet aggregation was not affected by $25 \mu \mathrm{g} / \mathrm{mL}$ of S-VWFA1 (Figure 2E). In addition, at this concentration, S-VWFA1 abolished platelet agglutination induced by R1306W VWF (Figure 2E).

\section{S-VWFA1 does not inhibit platelet binding to immobilized VWF}

The effect of S-VWFA1 on platelet-VWF interaction under shear stress was studied in two different assays. In a perfusion assay, S-VWFA1 at a concentration of $30 \mu \mathrm{g} / \mathrm{mL} \mathrm{did}$ not affect the adhesion of platelets (\% surface area covered by platelets) to collagen type I under high shear stress of 1600 s-1 (Figure 3A, C, D). Moreover, the mean fluores- 


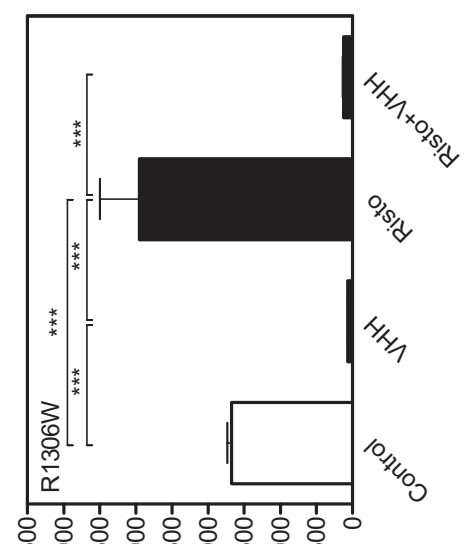

宅究 d

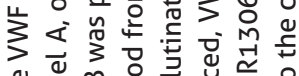

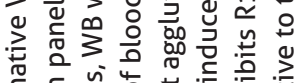

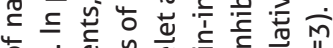
फั

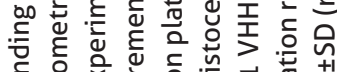
ํํำ

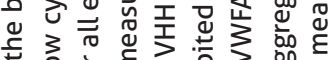

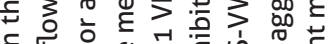

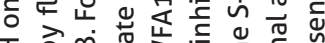

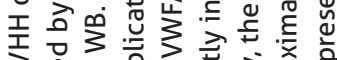

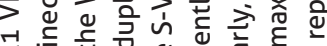

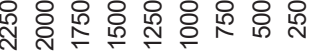

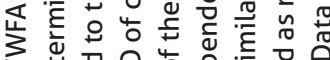

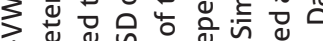
जे

$(\cap \forall)$ әวนәวรәлопџ uе!pəพ

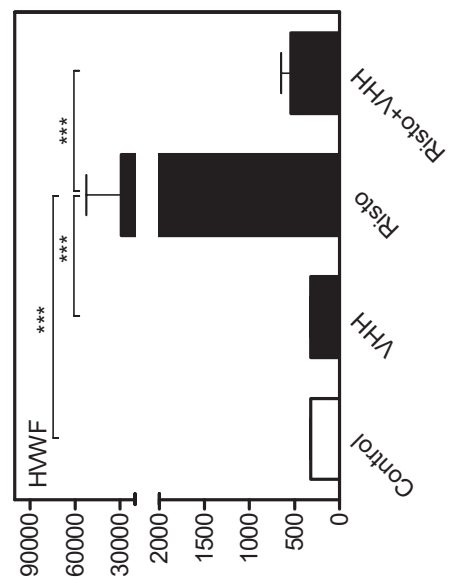

m

$(\cap \forall)$ әวนәวรәлопџ uе!pəพ

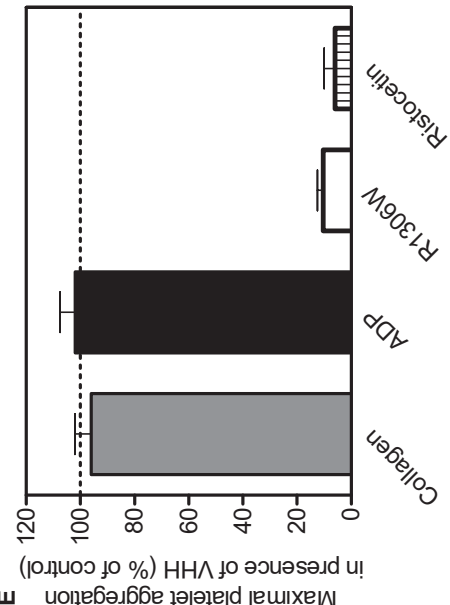

少

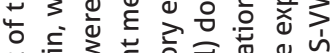

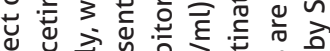

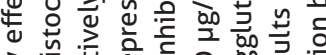

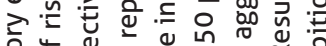

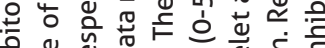

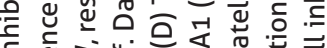
.

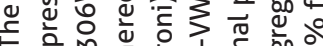

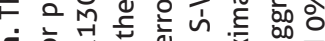
응 ᅯ 马

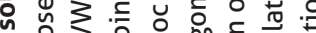

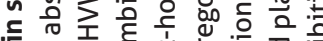
ทั 㫕 茴

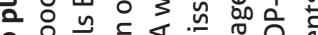

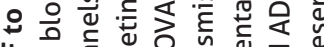

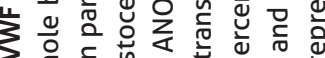
$\sum$ 은

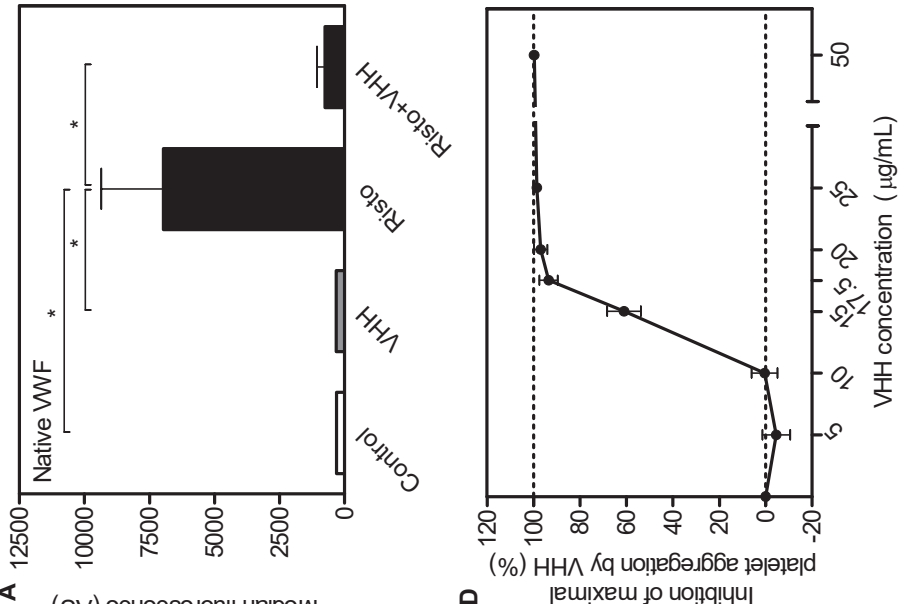
○ ט. 드 드 I

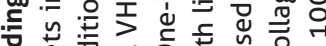

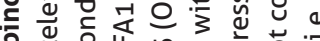
ย 背

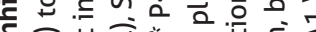

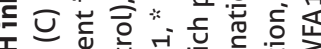
I

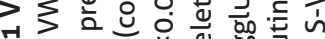

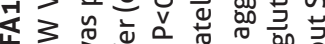

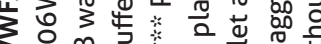

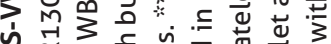

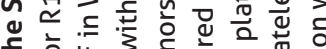

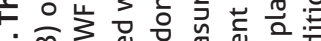
ヘ

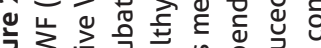

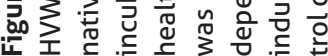



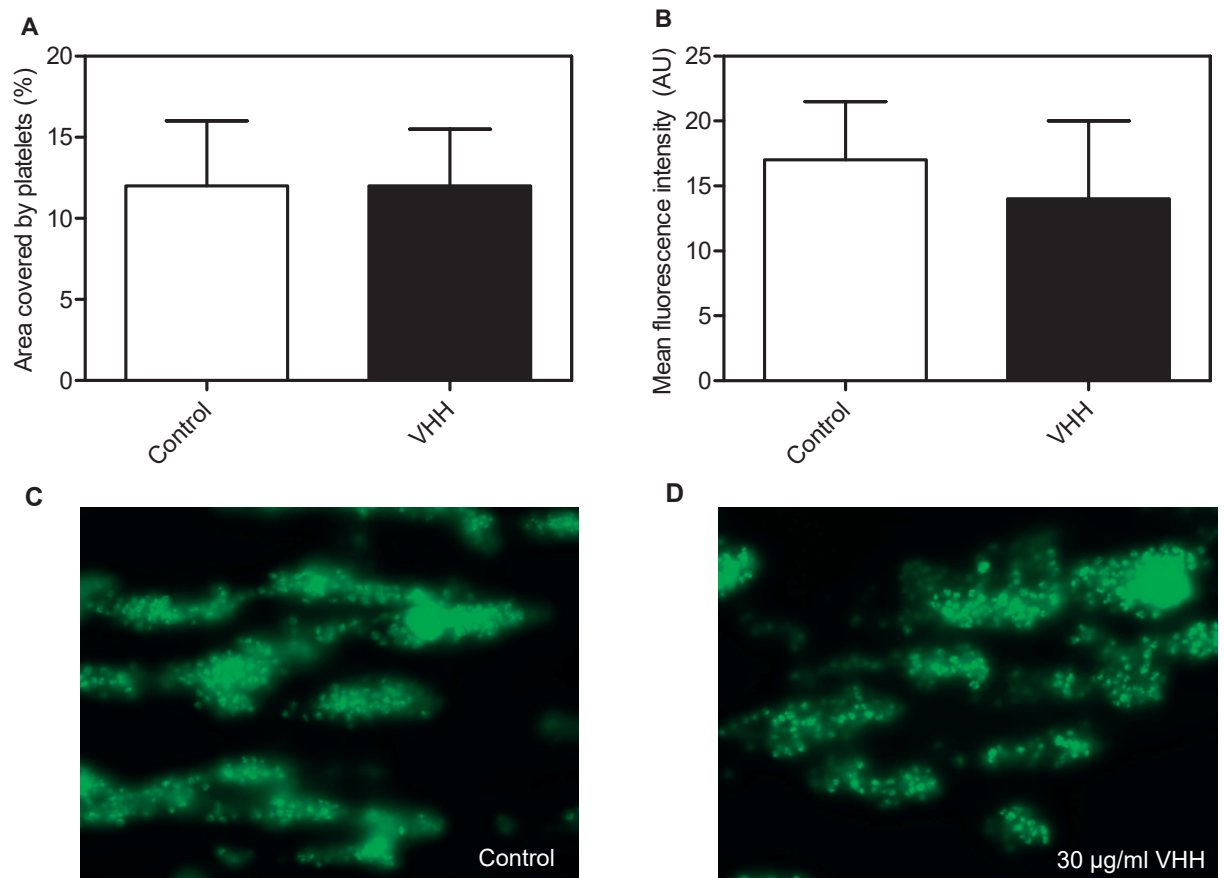

D
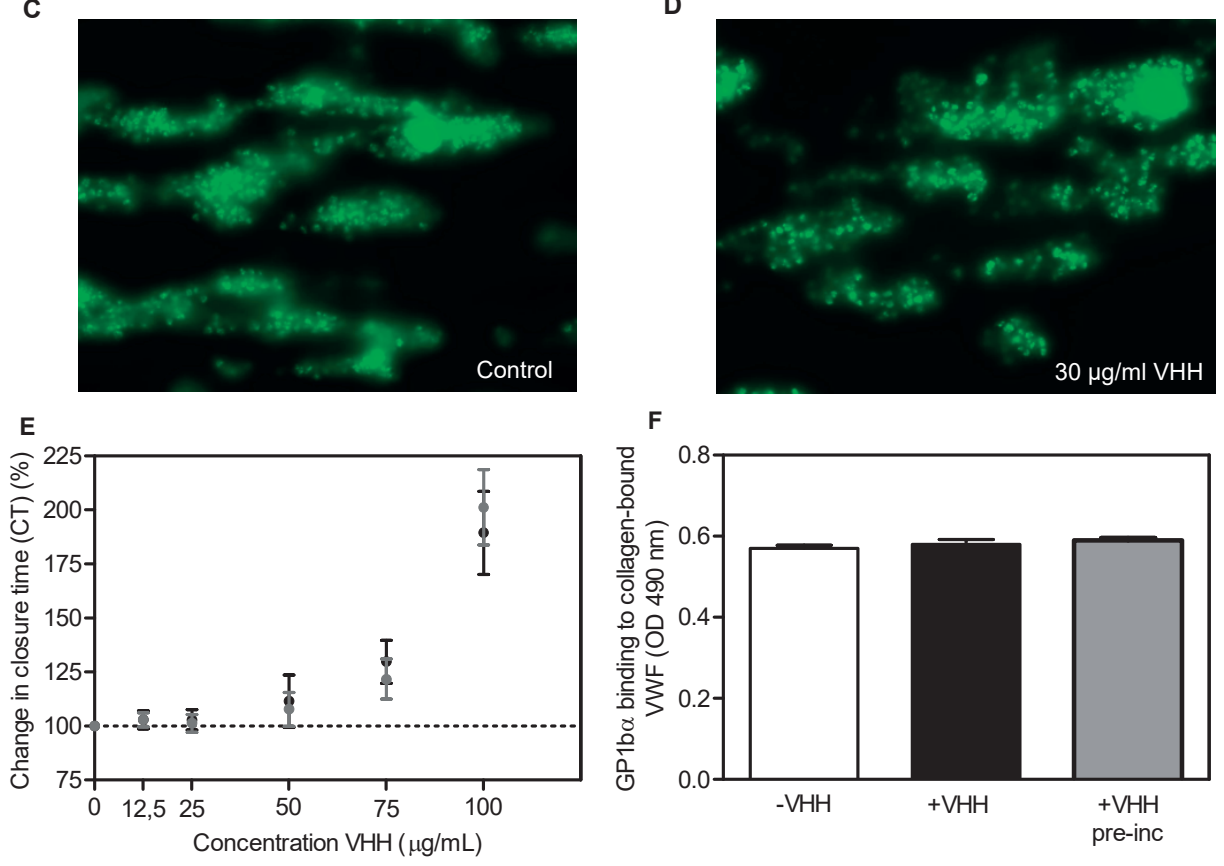

- Collagen/epinephrine - Collagen/ADP

Figure 3. S-VWFA1 does not inhibit platelet binding to immobilized VWF. (A-D) Whole blood with DiOC6-labeled platelets was perfused over immobilized collagen type I at a shear rate of 1600 $\mathrm{s}^{-1}$. Data represent mean \pm SD of 10 images taken after 4 minutes perfusion with whole blood from 5 healthy donors. (A) Quantification of the platelet covered area (\%), in the absence (white bar) and presence (black bar) of $30 \mu \mathrm{g} / \mathrm{mL}$ S-VWFA1 VHH. (B) The fluorescent intensity of platelet aggregates, in the absence (white bar) and presence (black bar) of $30 \mu \mathrm{g} / \mathrm{mL} \mathrm{S-VWFA1} \mathrm{VHH.} \mathrm{(C,} \mathrm{D)}$ Representative images of platelet aggregates (green/GFP) in the absence (C) and presence (D) of $30 \mu \mathrm{g} / \mathrm{mL}$ S-VWFA1 VHH. (E) Increasing concentrations (12.5-100 $\mu \mathrm{g} / \mathrm{mL}$ ) of S-VWFA1 VHH (or PBS as a control) were incubated with whole blood, followed by measurement of the aperture closure

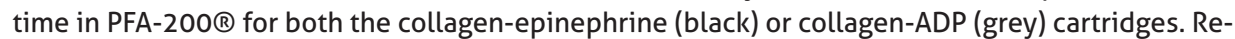
sults are expressed as \% change in closure time in the presence of S-VWFA1 VHH compared to the control (PBS), and represent the mean \pm SD of two replicates from 3 donors for each concentration. (F) HVWF activated by ristocetin $(0.5 \mathrm{mg} / \mathrm{mL})$ was pre-incubated with $50 \mu \mathrm{g} / \mathrm{mL} \mathrm{S}$-VWFA1 
VHH ("+VHH pre-inc" condition, grey bar) or saline ("-VHH" and "+VHH" conditions, white and black bar, respectively). This was added to collagen type III coated wells, followed by addition of saline (negative control), GP1b $\alpha$ (in the "+VHH pre-inc" and "-VHH" conditions) or a combination of GP1 $\alpha$ and $50 \mu \mathrm{g} / \mathrm{mL}$ S-VWFA1 ("+VHH" condition, black bar). Binding of GP1b $\alpha$ is expressed as the $O D($ mean $\pm S D)$ at $490 \mathrm{~nm}(\mathrm{n}=3)$.

cent intensity of the platelet aggregates was comparable in the absence and presence of S-VWFA1 (Figure 3B-D).

The concentration-dependency of the platelet-VWF interaction under shear stress was tested using the PFA-200® analyser. In the presence of collagen and either epinephrine or ADP as platelet agonists, S-VWFA1 could only prolong the closure time at considerably higher concentrations ( $>50 \mu \mathrm{g} / \mathrm{mL}$ ) than those used in the static assays described above (Figure 3E).

To test whether the reduced effect of S-VWFA1 on platelet-VWF binding in the perfusion system and PFA-200® assay was due to VWF immobilization, HVWF was activated by ristocetin, followed by binding to collagen type III pre-coated to a microtiter plate (Figure 3F). Under these static conditions, S-VWFA1 did not affect the binding of human GPIb $\alpha$ to collagen-immobilized HVWF (Figure 3F). This lack of an effect of S-VWFA1 was observed both when S-VWFA1 was pre-incubated with ristocetin-activated HVWF before addition to collagen, as well as when S-VWFA1 was added together with GPIb $\alpha$ after incubation of ristocetin-activated HVWF with collagen.

\section{DISCUSSION}

In the current study, we characterized the VHH S-VWFA1, targeted against a cryptic epitope in the VWF A1 domain, in terms of its ability to modulate the platelet-VWF interaction. Our results demonstrate that S-VWFA1 dose-dependently blocks the interaction of platelets to active VWF in solution, but not to active VWF either immobilized to collagen in a perfusion system or under static conditions in a microtiter plate.

S-VWFA1 could only efficiently interact with HVWF and VWF in NPP after conversion from the globular resting state to the activated state by treatment with ristocetin and by vortexing. Ristocetin binds to the proline-rich sequence Glu-1463-Asp-1472 ${ }^{11-13}$ (Figure 4), which alters electrostatic interactions and thereby exposes a GPIb-IX-V binding site ${ }^{14}$ possibly Asp-1277-Glu-1305. ${ }^{13,15}$ Vortexing NPP at high speed also increased the signal detected by S-VWFA1. This is consistent with previous work showing that the same vortex-induced shear (2,500 rpm) unfolds VWF to expose the A2 domain for 


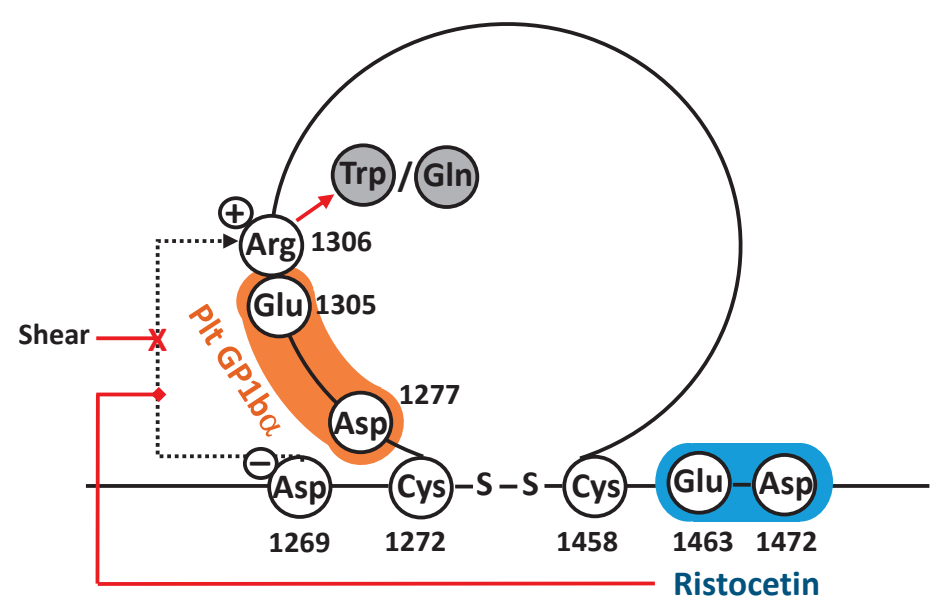

Figure 4. Scheme depicting the effects of ristocetin, shear stress and two "activating" mutations on the VWF A1 domain. Ristocetin binds to Glu-1463-Asp-1472, located C-terminal to the disulphide bond between Cys-1272 and Cys-1458. This interaction induces conformational changes that alter electrostatic interactions, such as the ionic bond (indicated by dashed arrow connecting the negative and positive charges) between Asp1269 and Arg1306, and thereby exposes a platelet (Plt) GPIb $\alpha$ binding site, likely Asp-1277-Glu-1305. Shear forces break the ionic bond between Asp1269 and Arg1306 causing the A1 domain to rotate and hence expose itself for interaction with Plt GPIb $\alpha$. Similarly, the R(Arg)1306W(Trp) and R(Arg)13060(Gln) mutations disrupt the ionic interaction with Asp1269, thereby facilitating the formation of a stable interaction with Plt GPIb $\alpha$.

cleavage by ADAMTS13. ${ }^{16}$ It was previously shown that the VWF-GPIb $\alpha$ interaction induced by ristocetin closely resembles the interaction induced by shear stress. ${ }^{17,18}$ Shear forces break the ionic bond between the Asp1269 and Arg1306 in the VWF A1 domain, causing the $\mathrm{A} 1$ domain to rotate and hence expose itself for interaction with $\mathrm{GPIb} \alpha .{ }^{19} \mathrm{Of}$ note, treatment with ristocetin did not further enhance the signal compared to vortexing alone, which suggests that these two mechanisms of VWF unfolding expose the S-VWFA1 epitope on the A1 domain of VWF to a similar extent.

Furthermore, we confirmed that S-VWFA1 readily bound to constitutively active R1306W and R13060 VWF. These mutations induce several conformational changes, resulting in improved binding to platelet GP1b $\alpha^{20,21}$, as well as increased sensitivity to shear stress $^{22}$ and ristocetin ${ }^{23}$ compared to wild-type VWF. Similar to shear stress, the R1306W mutation disrupts the ionic interaction with Asp1269, thereby facilitating the exposure of the A1 domain and the formation of a stable interaction with GPIb $\alpha .{ }^{22}$ Presumably, the loss of this specific ionic bond and the opening of the region between Asp1269 and Arg1306 may also facilitate S-VWFA1 binding to its epitope in the A1 domain. In contrast to our observations in vortex-opened HVWF and NPP VWF, pre-incubation with ristocetin did augment binding of S-VWFA1 to the recombinant R1306W and R13060 
VWF proteins. Thus, the combination of the R1306W or R13060 mutations with the ristocetin-induced conformational changes appears to provide optimal accessibility for S-VWFA1 to its epitope.

Interestingly, we found that S-VWFA1 strongly interfered with the interaction between platelets and active VWF in solution. In fact, in the flow cytometric platelet function assay, S-VWFA1 abolished binding of ristocetin-unfolded native VWF and HVWF to platelets, as well as spontaneous and ristocetin-induced binding of R1306W VWF to platelets. Moreover, S-VWFA1 dose-dependently inhibited ristocetin-induced platelet agglutination in LTA. These results suggest that, although the GP1b $\alpha$ and VHH epitopes in the VWF A1 domain may not be identical, binding of S-VWFA1 to its epitope hampers $\mathrm{GP} 1 \mathrm{~b} \alpha$-mediated platelet binding to its respective epitope.

However, when we investigated the effect of the same concentrations of S-VWFA1 in two different shear-dependent perfusion assays S-VWFA1 did not efficiently prevent platelet adhesion and aggregation. Given the inhibition of VWF-dependent platelet agglutination in the LTA and the platelet function assay we expected to see reduced platelet surface coverage and consequently reduced platelet aggregate formation in the perfusion system, as the initial step in the platelet adhesion process (GP1b $\alpha$-binding to unfolded VWF) would be blocked. However, pre-incubation of whole blood with SVWFA1 at $30 \mu \mathrm{g} / \mathrm{mL}$ prior to perfusion over collagen-coated channels neither affected platelet adhesion nor platelet aggregate formation. These observations corroborate those by Hulstein et al., who also observed no difference in platelet adhesion to collagen under the same high shear $\left(1600 \mathrm{~s}^{-1}\right)$ in the absence or presence of $(\sim 10 \mu \mathrm{g} / \mathrm{mL})$ AU/VWF-a11. ${ }^{6}$ We considered that the observed lack of inhibitory effect under shear stress may be due to rapid dilution of the small-molecular-weight ( $15 \mathrm{kDa}) \mathrm{VHH}$ in the perfusion system. Since a dose-response experiment in the perfusion assay would require very large amounts of S-VWFA1, we performed the dose-response experiment using the flow-based PFA-200® analyser. Closure of the aperture was only inhibited by S-VWFA1 at considerably higher concentrations ( $>50 \mu \mathrm{g} / \mathrm{mL}$ ) than required for full inhibition of platelet aggregation in LTA and the platelet function assay. While GP1b interaction with shear-unfolded VWF contributes to retarding blood flow in the PFA$200 \circledR$ assay, inhibition of the GPIlbllla-mediated platelet aggregation is also a major determinant of aperture closure. ${ }^{24}$ It may be hypothesized that S-VWFA1 cannot inhibit initial platelet-binding to the VWF immobilized to the collagen-coated on the aperture, but at extremely high concentrations it seems to interfere with secondary platelet aggregation and hence with the closure of the aperture. 
We considered several factors/conditions that may be critical for S-VWFA1 to exert its inhibiting function. First of all, whereas in the PFA-200@ and perfusion assays unfolding of VWF is triggered by shear stress, this process is triggered by ristocetin in LTA and the flow cytometric platelet function assay. This raises the question whether exposure of the S-VWFA1 epitope through the unfolding of VWF induced by ristocetin is more efficient than by shear stress. However, in the immunosorbent assay, both ristocetin and vortexing induced similar binding of S-VWFA1 to VWF in NPP. Secondly, we considered that high shear may rapidly wash away, or interfere with reversible binding of the small-molecular weight ( $15 \mathrm{kDa}) \mathrm{VHH}$ to its epitope. However, arguing against this reasoning is the observation by Hulstein et al. that, in contrast to AU/VWF-a11, a control nanobody (of similar molecular weight), raised against the isolated A1 domain, did effectively inhibit platelet adhesion under the same shear conditions. ${ }^{6}$ The third factor we hypothesized may disturb the function of S-VWFA1 is immobilization to collagen. In our experiment, S-VWFA1 did not affect platelet adhesion to collagen I-bound VWF under shear stress. Furthermore, Hulstein et al. demonstrated that the AU/VWF-a11 VHH also did not affect platelet adhesion to collagen III-bound VWF nor to a VWF-coated surface under the same flow conditions. ${ }^{6}$ We confirmed this lack of an inhibitory effect of SVWFA1 on the binding of Gplba to collagen-bound VWF (and VWF coated to a plate) under static conditions. Although VWF domains $A 1$ and $A 3$ both contain binding sites for collagen, the major binding site for collagens I and III resides in A3 (aa 1683-1874), while the A1 domain contains the primary binding sequence for collagen $\mathrm{VI} .{ }^{25,26}$ Importantly, the isolated A1 domain can simultaneously interact with collagen and GPIb $\alpha$, allowing platelet recruitment under flow conditions. ${ }^{27}$ Moreover, A1 binding to collagen was previously shown to induce a conformational change in $\mathrm{A} 1 .{ }^{27}$ This conformational change may be responsible for blocking the $\mathrm{VHH}$ epitope and hence for the lack of an inhibiting effect of S-VWFA1 on platelet-binding to collagen-immobilized VWF.

In conclusion, these findings suggest that the immobilization of VWF to collagen affects VWF conformation in such a way that access of S-VWFA1 to its epitope in the A1 domain is hindered, while allowing platelet binding. If this hypothesis holds, it implies that S-VWFA1 has beneficial properties to be studied further as a potential treatment for conditions with increased circulating active VWF, as it may be able to prevent pathological platelet-VWF aggregate formation while leaving physiological haemostasis at sites of vascular injury (where VWF immobilizes to collagen) intact. 


\section{ACINOWLEDGEMENTS}

The authors would like to thank Prof. J.W.M. Heemskerk for use of the EVOS FL microscope and M. Visser-Koldenhof for assistance with the LTA experiments. 


\section{REFERENCES}

1. Springer TA. von Willebrand factor, Jedi knight of the bloodstream. Blood. 2014;124(9):141225.

2. Huizinga EG, Tsuji S, Romijn RA, Schiphorst ME, de Groot PG, Sixma JJ, et al. Structures of glycoprotein Ibalpha and its complex with von Willebrand factor A1 domain. Science. 2002;297(5584):1176-9.

3. Groot E, de Groot PG, Fijnheer R, Lenting PJ. The presence of active von Willebrand factor under various pathological conditions. Curr Opin Hematol. 2007;14(3):284-9.

4. Ruggeri ZM, Zimmerman TS. von Willebrand factor and von Willebrand disease. Blood. 1987;70(4):895-904.

5. Sharma R, Flood VH. Advances in the diagnosis and treatment of Von Willebrand disease. Hematology Am Soc Hematol Educ Program. 2017;2017(1):379-84.

6. Hulstein JJ, de Groot PG, Silence K, Veyradier A, Fijnheer R, Lenting PJ. A novel nanobody that detects the gain-of-function phenotype of von Willebrand factor in ADAMTS13 deficiency and von Willebrand disease type 2B. Blood. 2005;106(9):3035-42.

7. Sadler JE. Von Willebrand factor, ADAMTS13, and thrombotic thrombocytopenic purpura. Blood. 2008;112(1):11-8.

8. Lenting PJ, Christophe OD, Denis CV. von Willebrand factor biosynthesis, secretion, and clearance: connecting the far ends. Blood. 2015;125(13):2019-28.

9. Scully M, Cataland SR, Peyvandi F, Coppo P, Knobl P, Kremer Hovinga JA, et al. Caplacizumab Treatment for Acquired Thrombotic Thrombocytopenic Purpura. N Engl J Med. 2019;380(4):335-46.

10. Lankhof H, Damas C, Schiphorst ME, MJ IJ, Bracke M, Sixma JJ, et al. Functional studies on platelet adhesion with recombinant von Willebrand factor type 2B mutants R5430 and R543W under conditions of flow. Blood. 1997;89(8):2766-72.

11. Azuma $H$, Sugimoto $M$, Ruggeri ZM, Ware J. A role for von Willebrand factor proline residues 702-704 in ristocetin-mediated binding to platelet glycoprotein lb. Thromb Haemost. 1993;69(2):192-6.

12. Girma JP, Takahashi Y, Yoshioka A, Diaz J, Meyer D. Ristocetin and botrocetin involve two distinct domains of von Willebrand factor for binding to platelet membrane glycoprotein lb. Thromb Haemost. 1990;64(2):326-32.

13. Berndt MC, Ward CM, Booth WJ, Castaldi PA, Mazurov AV, Andrews RK. Identification of aspartic acid 514 through glutamic acid 542 as a glycoprotein Ib-IX complex receptor recognition sequence in von Willebrand factor. Mechanism of modulation of von Willebrand factor by ristocetin and botrocetin. Biochemistry. 1992;31(45):11144-51.

14. Kang M, Wilson L, Kermode JC. Evidence from limited proteolysis of a ristocetin-induced conformational change in human von Willebrand factor that promotes its binding to platelet glycoprotein Ib-IX-V. Blood Cells Mol Dis. 2008;40(3):433-43.

15. De Luca M, Facey DA, Favaloro EJ, Hertzberg MS, Whisstock JC, McNally T, et al. Structure and function of the von Willebrand factor A1 domain: analysis with monoclonal antibodies reveals distinct binding sites involved in recognition of the platelet membrane glycoprotein Ib-IX-V complex and ristocetin-dependent activation. Blood. 2000;95(1):164-72.

16. Han Y, Xiao J, Falls $E$, Zheng $X L$. A shear-based assay for assessing plasma ADAMTS13 activity and inhibitors in patients with thrombotic thrombocytopenic purpura. Transfusion. 2011;51(7):1580-91. 
17. Shen Y, Romo GM, Dong JF, Schade A, McIntire LV, Kenny D, et al. Requirement of leucine-rich repeats of glycoprotein (GP) Ibalpha for shear-dependent and static binding of von Willebrand factor to the platelet membrane GP Ib-IX-V complex. Blood. 2000;95(3):903-10.

18. Dong JF, Berndt MC, Schade A, McIntire LV, Andrews RK, Lopez JA. Ristocetin-dependent, but not botrocetin-dependent, binding of von Willebrand factor to the platelet glycoprotein Ib-IX-V complex correlates with shear-dependent interactions. Blood. 2001;97(1):162-8.

19. Yago T, Lou J, Wu T, Yang J, Miner JJ, Coburn L, et al. Platelet glycoprotein lbalpha forms catch bonds with human WT vWF but not with type 2B von Willebrand disease vWF. J Clin Invest. 2008;118(9):3195-207.

20. Dumas J], Kumar R, McDonagh T, Sullivan F, Stahl ML, Somers WS, et al. Crystal structure of the wild-type von Willebrand factor A1-glycoprotein Ibalpha complex reveals conformation differences with a complex bearing von Willebrand disease mutations. J Biol Chem. 2004;279(22):23327-34.

21. Siedlecki CA, Lestini BJ, Kottke-Marchant KK, Eppell SJ, Wilson DL, Marchant RE. Sheardependent changes in the three-dimensional structure of human von Willebrand factor. Blood. 1996;88(8):2939-50.

22. Scaglione GL, Lancellotti S, Papi M, De Spirito M, Maiorana A, Baronciani L, et al. The type 2B p.R1306W natural mutation of von Willebrand factor dramatically enhances the multimer sensitivity to shear stress. J Thromb Haemost. 2013;11(9):1688-98.

23. Ruggeri ZM, Pareti FI, Mannucci PM, Ciavarella N, Zimmerman TS. Heightened interaction between platelets and factor VIII/von Willebrand factor in a new subtype of von Willebrand's disease. N Engl J Med. 1980;302(19):1047-51.

24. Watala C, Golanski J, Rozalski M, Boncler MA, Luzak B, Baraniak J, et al. Is platelet aggregation a more important contributor than platelet adhesion to the overall platelet-related primary haemostasis measured by PFA-100? Thromb Res. 2003;109(5-6):299-306.

25. Lankhof $H$, van Hoeij $M$, Schiphorst ME, Bracke $M$, Wu YP, ljsseldijk MJ, et al. A3 domain is essential for interaction of von Willebrand factor with collagen type III. Thromb Haemost. 1996;75(6):950-8.

26. Mazzucato M, Spessotto P, Masotti A, De Appollonia L, Cozzi MR, Yoshioka A, et al. Identification of domains responsible for von Willebrand factor type VI collagen interaction mediating platelet adhesion under high flow. J Biol Chem. 1999;274(5):3033-41.

27. Morales LD, Martin C, Cruz MA. The interaction of von Willebrand factor-A1 domain with collagen: mutation $\mathrm{G} 1324 \mathrm{~S}$ (type $2 \mathrm{M}$ von Willebrand disease) impairs the conformational change in A1 domain induced by collagen. J Thromb Haemost. 2006;4(2):417-25. 


$$
\text { Chapter } 3
$$




\section{Analytical characterization and reference interval of an enzyme-linked immunosorbent assay for active von Willebrand factor}

Van der Vorm LN, Li L, Huskens D, Chayoua W, Kelchtermans H, de Groot $P G$, Roest M, Remijn JA, de Laat $B$ 


\section{ABSTRACT}

Background - Interaction of von Willebrand factor (VWF) with platelets requires a conformational change that exposes an epitope within the VWF A1 domain, enabling platelet glycoprotein $\mathrm{lb} \alpha$ binding. Quantification of this active conformation of von Willebrand Factor (VWF) has been shown to provide pathophysiological insight into conditions characterized by excessive VWF-platelet interaction.

Methods - We developed an immunosorbent assay based on a variable heavy chain antibody fragment against the VWF A1 domain as a capture antibody. Assay performance in terms of specificity, precision, accuracy, linearity, limits of detection and stability were determined. Active VWF, VWF antigen, VWF ristocetin cofactor activity, VWF:GP1bM and VWF propeptide were measured in citrated plasma and platelet-VWF binding in whole blood from 120 healthy individuals to establish a reference interval for active VWF and to assess associations with other VWF parameters.

Results - Intra- and inter-assay CVs were between 2.4-7.2\% and 4.1-9.4\%, depending on the level. Mean recovery of spiked recombinant R1306W VWF was $103 \pm 3 \%$. The assay was linear in the range of $90.1-424.5 \%$ and had a limit of quantification of $101 \%$. The reference interval for active VWF was $91.6-154.8 \%$ of NPP. Significant, positive correlations between active VWF and all other VWF parameters were found, with the strongest correlation with VWF:GP1bM binding.

Conclusions - We developed and validated an immunosorbent assay for the accurate detection of active VWF levels in plasma. The assay fulfilled all analytical criteria in this study and a reference interval was established, allowing its use to quantify active VWF in pathological conditions for future research. 


\section{INTRODUCTION}

Von Willebrand factor (VWF) is a multimeric plasma protein that mediates platelet adhesion and platelet-platelet interactions. ${ }^{1}$ VWF binds via its A3 domain to exposed subendothelial collagen at sites of vascular injury. Collagen-bound VWF tethers platelets to the vessel wall via transient interaction of its $A 1$ domain with the platelet glycoprotein (GP)Ib-IX-V receptor complex. ${ }^{2}$ Circulating VWF can only exert this function after conversion from its latent, globular conformation to an active conformation, in which the binding site for platelet Gplba is exposed. Under physiological conditions, conversion to this active state is well regulated. Upon vascular injury, VWF immobilization to subendothelial collagen in conjunction with increased shear stress induce VWF unfolding $^{3}$, allowing for platelet-VWF interaction. ${ }^{4}$

Various pathological conditions are associated with premature and/or excessive formation of VWF-platelet aggregates. ${ }^{5}$ Von Willebrand disease (VWD) type $2 \mathrm{~B}$, for instance, is characterized by increased interactions between VWF and platelets, resulting from gain-of-function mutations (e.g. R1306W) in the VWF A1 domain. ${ }^{6}$ Consequently, these patients lack high-molecular-weight VWF multimers and suffer from thrombocytopenia, clinically resulting in a bleeding phenotype. ${ }^{7}$ In thrombotic thrombocytopenic purpura (TTP) patients, an acquired or inherited deficiency of the VWF cleaving protease ADAMTS13 results in the accumulation of ultra-large (UL)-VWF multimers. ${ }^{8}$ Clinically the result is a thrombotic phenotype caused by platelet-rich thrombi occluding the microvasculature. ${ }^{8}$ Spontaneous VWF-platelet interaction in these conditions is indicative of the presence of VWF in its active conformation.

Several laboratory tests to assess VWF are available. The VWF antigen (VWF:Ag) assay is a quantitative assay that provides an overall level of VWF present in plasma, but yields no information concerning the quality of VWF in terms of its ability to bind platelets. ${ }^{9}$ The ristocetin cofactor (VWF:RCo) assay is the most widely used method to assess the functional activity of VWF. ${ }^{10}$ However, VWF:RCo assays are highly variable ${ }^{11}$ and the need for ristocetin to activate VWF does not reflect the in vivo situation. A more recently developed assay uses recombinant GPIb fragments with two gain-of-function mutations that allow binding to VWF in the absence of ristocetin (VWF:GPIbM). ${ }^{12}$ Although this VWF:GPIbM assay eliminates the need for the non-physiological activator ristocetin, it is based on non-physiological binding of VWF to a mutant receptor.

Therefore, an immunosorbent assay to directly detect circulating VWF in its active conformation was previously developed. ${ }^{13}$ This assay is based on a recombinant llama-derived antibody (AU/VWFa-11) that preferentially binds the Gplb $\alpha$-binding conformation 
of the VWF A1 domain. ${ }^{13}$ Using this assay, elevated active VWF levels were identified in plasma from patients with VWD type $2 \mathrm{~B}^{13}, \mathrm{TTP}^{13}$, HELLP syndrome ${ }^{14}$, systemic inflammatory response syndrome ${ }^{15}$, antiphospholipid syndrome ${ }^{16}$, diabetes ${ }^{17}$, first ST-segment elevation myocardial infarction (STEMI ${ }^{18}$, sickle cell disease ${ }^{19}$, malaria ${ }^{20}$ and dengue ${ }^{21}$. Together, these findings have provided new insights into the presence of, as well as the mechanism behind increased active VWF in these pathological conditions. Moreover, quantification of active VWF has potential value for diagnostics, for example to differentiate VWD 2B from other types of VWD, to distinguish between forms of VWD 2B and to differentiate between patients with acquired and congenital TTP. ${ }^{13,22}$

However, there are no reports of an analytical validation of this assay. Therefore, we developed our own in-house immunosorbent assay to measure active VWF, also based on a llama-derived variable heavy chain antibody fragment (VHH) directed against a cryptic epitope in the A1 domain of VWF. The aims of the current study were to (1) characterize the analytical performance of this assay; (2) provide a reference interval for active VWF and (3) determine correlations between this assay and other, established VWF assays and a whole blood flow cytometric assay for platelet-VWF binding (Plt:VWF binding ${ }^{23-25}$ ).

\section{MATERIALS AND METHODS}

\section{Ethics statement}

The research complied with all the relevant national regulations, institutional policies and the tenets of the Helsinki Declaration, and has been evaluated by the Medical Ethical Committee (METC) of Maastricht University Medical Center/University of Maastricht (METC reference 152015). Participants gave full written informed consent.

\section{Reagents}

Haemate P (CSL Behring, Pennsylvania, USA) VWF/FVIII concentrate (stock $12 \mathrm{mg} / \mathrm{mL}$ ) was used as native human VWF in its globular conformation, referred to as "HVWF". Recombinant VWF with the VWD type 2B R1306W (stock $17 \mu \mathrm{g} / \mathrm{mL}$ ) or R13060 (stock 55 $\mu \mathrm{g} / \mathrm{mL}$ ) mutation (produced as described previously ${ }^{26}$ ) was used as VWF constitutively in its active conformation. Polyclonal rabbit anti-VWF antibody conjugated to HRP was purchased from Dako (P0226, Glostrup, Denmark). Bovine serum albumin (BSA; A7906), SIGMAFAST o-Phenylenediamine dihydrochloride (OPD; P9187) and sulfuric acid $\left(\mathrm{H}_{2} \mathrm{SO}_{4}\right.$; 339741) were all purchased from Sigma-Aldrich (Zwijndrecht, The Netherlands). 


\section{Production of VHH}

See Supplemental Methods for a detailed description of VHH production.

\section{Study population and plasma preparation}

The study population consisted of 120 healthy individuals, aged 18-65 years, recruited between March 12, 2018 and May 17, 2018. Participants did not take any anti-coagulant or anti-platelet drugs for at least one week and did not have a history of thrombosis or bleeding. Analytical characterization of the assay was performed using residual, anonymized citrated plasma from the reference value study samples as well as residual, anonymized citrated plasma initially collected for routine laboratory diagnostics conducted at the Gelre Hospitals Apeldoorn, the Netherlands. Blood was collected by antecubital venepuncture into vacuum tubes ( 1 volume trisodium citrate $0.105 \mathrm{M}$ to 9 volumes blood) (BD Vacutainer System, Becton Dickinson, Franklin Lakes, USA). Cell counts in whole blood were performed with a Coulter Counter analyser (Beckman Coulter, Woerden, The Netherlands). Plasma was prepared by centrifugation at 2,840 $\mathrm{g}$ for 10 minutes, pipetting off the plasma fraction, and repeating centrifugation to obtain the platelet-poor plasma fraction, which was stored at $-80^{\circ} \mathrm{C}$.

\section{Active VWF immunosorbent assay}

The active VWF assay is based on a llama-derived single-domain antibody (VHH) directed against a cryptic epitope in the A1 domain of VWF (Figure 1), which is only exposed upon unfolding of VWF. ${ }^{13}$ Briefly, 96 wells microtiter plates (NUNC Maxisorp, Thermo Fisher Scientific, Waltham, USA) were coated overnight at $4^{\circ} \mathrm{C}$ with $1.98 \mu \mathrm{g} / \mathrm{ml}$ VHH against active VWF in a carbonate-bicarbonate coating buffer ( $\mathrm{pH} 9.6$ ), followed by a blocking step with $2 \%$ BSA in phosphate-buffered saline (PBS) for 45 minutes at room temperature (RT). After washing with $0.01 \%$ Tween-20 in PBS, plasma samples (diluted $1: 20$ in PBS/1\% BSA) were incubated for 2 hours at RT. Following another wash-step, the wells were incubated with HRP-conjugated anti-VWF antibodies $(1.2 \mu \mathrm{g} / \mathrm{mL})$ in PBS/1\% BSA for 2 hours at room temperature. Plates were then washed again before the addition of SIGMAFAST OPD (Sigma). The reaction was stopped with $3 \mathrm{M}$ sulfuric acid $\left(\mathrm{H}_{2} \mathrm{SO}_{4 \prime}\right.$ Sigma). Optical densities (OD) were measured at $490 \mathrm{~nm}$ using an ELx808 Absorbance Microplate Reader (Biotek, Bad Friedrichshall, Germany). Results were normalized (\%) to normal pooled plasma (NPP) on the same plate. This differs from the quantification method in earlier studies using the previous version of this assay, reporting the VWF activation factor. ${ }^{13}$ The VWF activation factor was calculated by dividing the absorbance slope of the dilution curves for a patient sample by the slope of an NPP sample. We simplified the assay and reduced the required sample volume by measuring one dilution of each plasma sample and expressing its active VWF level compared to that in NPP. For the NPP, blood from 10 healthy control donors was collected. After double centrifugation 
(2,840 $\mathrm{g}$ for $10 \mathrm{~min})$, plasmas were pooled and aliquots of $1 \mathrm{ml}$ were stored at $-80{ }^{\circ} \mathrm{C}$ until use in the active VWF ELISA. Of note, NPP contains only a very small amount of active VWF. If an individual has an active VWF level of $150 \%$, he/she has 1.5 times more circulating active VWF than is present in the NPP, which is still a minute amount, but the current assay is sensitive enough to detect this difference.

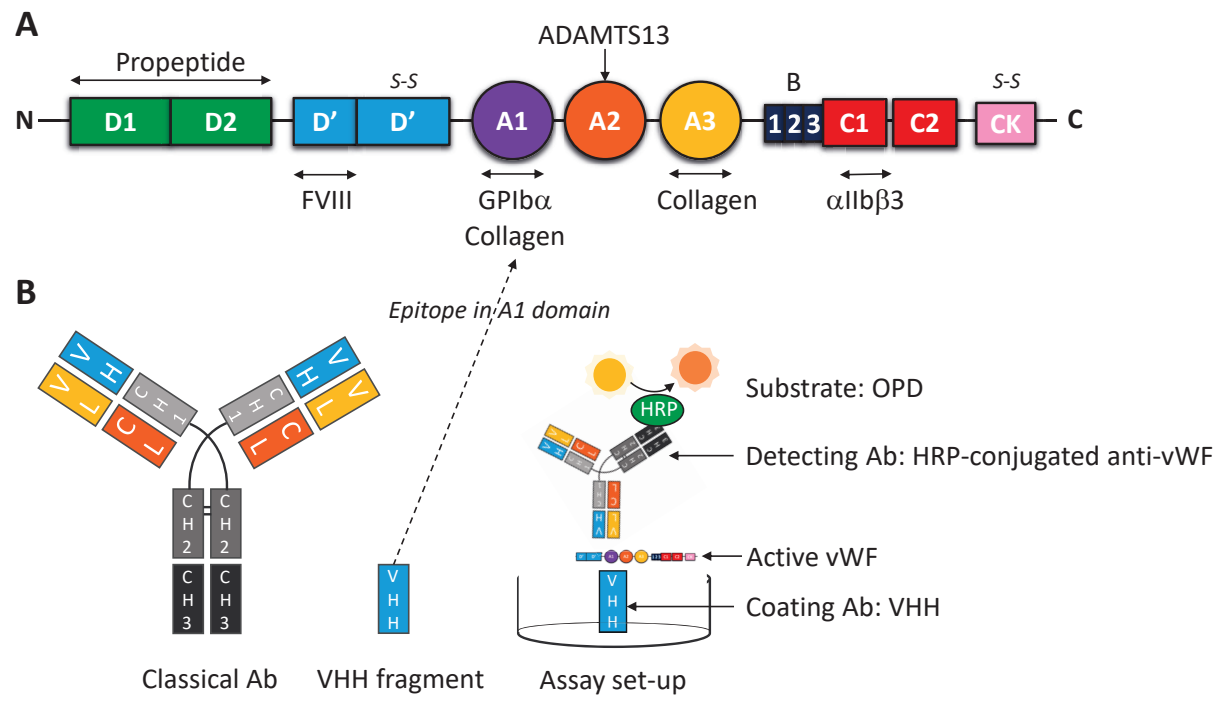

Figure 1. Scheme for measurement of active VWF. (A) The protein domain structure of mature VWF (adapted from Crawley et al. ${ }^{29}$ ). (B) The set-up of the immunosorbent assay described here, based on a variable heavy chain antibody fragment (VHH) directed against a cryptic epitope in the A1 domain of VWF as capture antibody, and a polyclonal rabbit anti-human VWF antibody conjugated with HRP as detecting antibody.

\section{Assay performance studies}

See Supplemental Methods for a detailed description of the assay performance studies.

\section{Established VWF parameters}

VWF antigen (VWF:Ag) levels were determined using the Liatest VWF assay on an automated STA-R Max coagulation analyser (Diagnostica Stago, Leiden, the Netherlands). VWF ristocetin cofactor activity (VWF:RCo) was measured with the automated chemiluminescent HemosIL VWF:RCo assay (Werfen-Instrumentation Laboratory, Bedford, USA). VWF propeptide (VWFpp) was measured using the anti-human VWFpp MW1939 antibody pair and Tool Set 2 (Sanquin, Amsterdam, The Netherlands) ${ }^{27}$ according to the manufacturer instructions, with the exception that VWFpp was expressed as a \% of the level in NPP included on the same plate (due to the limited number of available kits the recommended 8 step 2 -fold serial dilution curve could not be included) VWF:GP1bM 
was measured using the INNOVANCE VWF Ac assay on a Sysmex CS-2500 instrument (Siemens Healthcare Diagnostics $\mathrm{GmbH}$, Marburg, Germany) according to the manufacturer's protocol.

\section{Flow cytometric analysis of Plt:VWF binding}

See Supplemental Methods for a detailed description of this assay, for which standardisation and a reference range were described previously. ${ }^{28}$

\section{Statistical analysis}

Outlier analysis was performed using Tukey's hinges. One outlier in the active VWF values was detected (167.9\%) but there was no evidence of an experimental error and this value was hence not excluded. Normality was tested using Q-O plots and the Shapiro-Wilk test. None of the VWF parameters were normally distributed. Continuous variables were expressed as the median and interquartile range (25\%-75\%). Reference intervals were obtained using nonparametric calculation, according to the latest CLSI guidelines ${ }^{30}$, i.e. the $2.5^{\text {th }}$ percentile to the $97.5^{\text {th }}$ percentile of the distribution. Associations between variables were determined using Spearman's rank correlation coefficient. Groups were compared using the Mann-Whitney $U$ test for independent samples (continuous variables) or the Chi-square test for categorical variables. Multiple linear regression was used to adjust for the effect of differences in VWF:Ag levels on associations between (1) blood group and VWF:RCo, VWF:GP1bM and Plt:VWF binding, and (2) sex and active VWF levels. A p-value of 0.05 was considered statistically significant for all comparisons. Analyses were performed using Statistical Package for Social Sciences (SPSS Incorporate, Chicago, USA) version 25. Figures were prepared using GraphPad Prism version 5.00 (GraphPad Software, San Diego, USA).

\section{RESULTS}

\section{VHH specifically detects active VWF}

To test the specificity of the VHH for the active conformation of VWF, its binding to HVWF, R1306W VWF, R13060 VWF, and VWF in NPP was investigated (Figure 2A). The VHH neither interacted with HVWF nor VWF in NPP, as apparent from the weak (background) signal at $490 \mathrm{~nm}$. In contrast, binding of the VHH to constitutively active R1306W and R13060 VWF resulted in a strong dose-dependent signal. To confirm that the VHH also recognizes native VWF that is activated by high shear stress we also tested vortexed NPP. As a result, the VHH interacted with the shear-unfolded VWF in NPP. Thus, the VHH specifically recognizes VWF in its active conformation. 
A

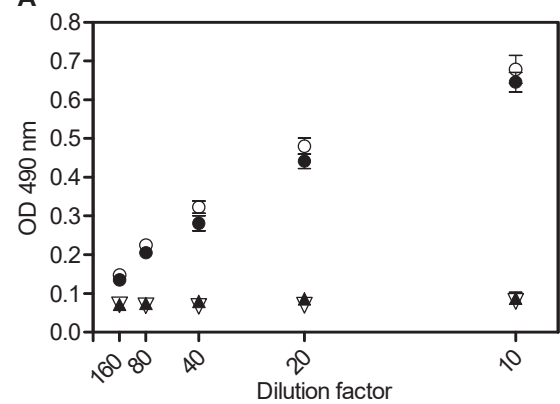

C

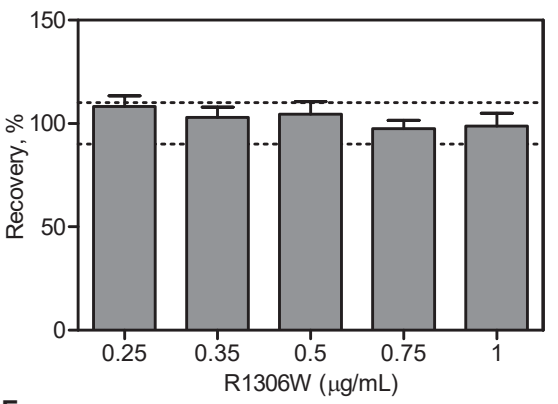

E

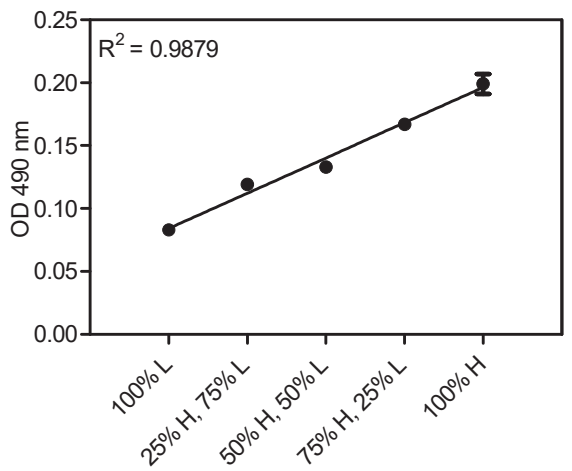

B

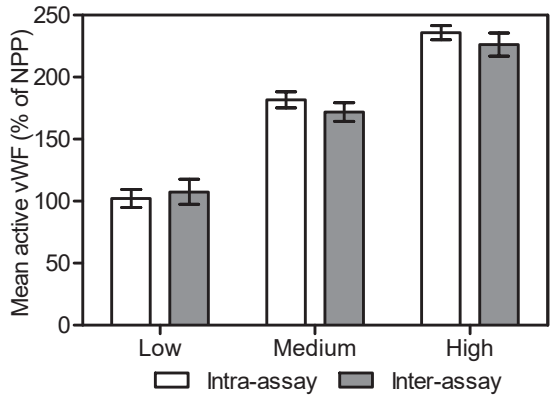

D

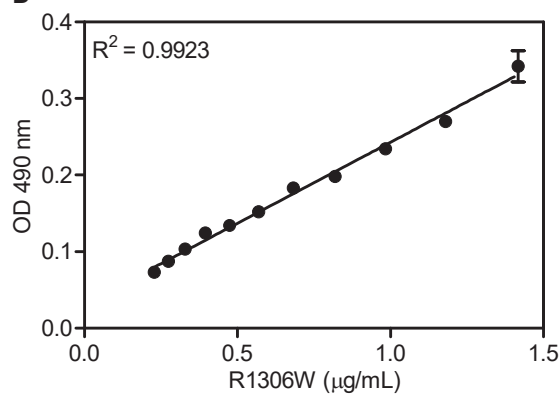

$\mathbf{F}$

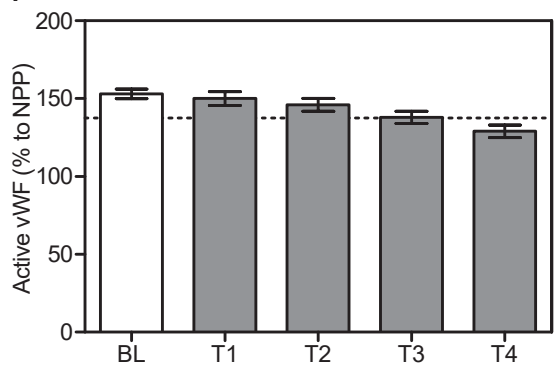

Figure 2. Analytical characteristics of the active VWF assay. (A) Binding of the VHH to R1306W VWF (•), R13060 VWF (o), HVWF (s), VWF in NPP under static conditions (p) and NPP after vortexing to simulate shear stress ( $\S$, only $10 x$ diluted). Dilution factors are indicated at the $x$-axis. (B) Mean active VWF levels in plasma pools (with low (91-110\%), medium (150-200\%) and high (>220\%) active VWF levels) with corresponding SDs for 20 replicates on a single plate on the same day (intra-assay, white bars) or on 20 separate days (inter-assay, grey bars). (C) Recovery (\%) of increasing levels of R1306W VWF spiked to plasma pool. The acceptance range of $90-110 \%$ of expected values is indicated by dotted lines. (D) Assay response (OD at $490 \mathrm{~nm}$ ) for a 12-step, 1.2fold dilution series of plasma spiked with R1306W. Linear regression parameters $( \pm S D)$ are: slope $0,21 \pm 0.006$, intercept $0.03 \pm 0.005$, SD of residuals 0.007 . $(\mathrm{E})$ Low $(\mathrm{L})$ and high $(\mathrm{H})$ active VWF plasma pools were mixed in varying proportions to assess dilutional linearity. (F) Stability of active VWF in a plasma pool at baseline and after multiple freeze-thaw steps (Thaw 1-4). The acceptance criterium of $<10 \%$ analyte loss is indicated by the dotted line. Data represent the mean $\pm S D(n=3)$ unless otherwise stated. 
Table 1. Analytical performance characteristics of the active VWF ELISA assay.

\begin{tabular}{|c|c|c|c|c|c|c|c|}
\hline \multirow{2}{*}{$\begin{array}{l}\text { Parameter } \\
\text { Precision }\end{array}$} & \multicolumn{7}{|c|}{ Active VWF assay performance } \\
\hline & \multicolumn{4}{|c|}{ Intra-assay (Mean [SD;\%CV]) } & \multicolumn{3}{|c|}{ Inter-assay (Mean [SD;\%CV]) } \\
\hline & L & $102 \%$ & \multicolumn{2}{|c|}{$[7.3 \% ; 7.2 \%]$} & $\mathrm{L}$ & $107 \%$ & {$[10.1 \% ; 9.4 \%]$} \\
\hline & M & $182 \%$ & \multicolumn{2}{|c|}{$[6.6 \% ; 3.7 \%]$} & M & $172 \%$ & {$[7.6 \% ; 4.4 \%]$} \\
\hline & $\mathrm{H}$ & $235 \%$ & \multicolumn{2}{|c|}{$[5.7 \% ; 2.4 \%]$} & $\mathrm{H}$ & $226 \%$ & {$[9.2 \% ; 4.1 \%]$} \\
\hline \multirow[t]{6}{*}{ Accuracy } & \multicolumn{2}{|l|}{ Spiked } & {$[E]_{O D}$} & {$[O]_{O D}$} & {$[E]_{\%}$} & {$[0]_{\%}$} & $\operatorname{Rec} \%$ \\
\hline & \multicolumn{2}{|c|}{$1.0 \mu \mathrm{g} / \mathrm{mL}$} & 0.233 & 0.230 & 333 & 329 & 99 \\
\hline & \multicolumn{2}{|c|}{$0.75 \mu \mathrm{g} / \mathrm{mL}$} & 0.175 & 0.171 & 250 & 245 & 98 \\
\hline & \multicolumn{2}{|c|}{$0.5 \mu \mathrm{g} / \mathrm{mL}$} & 0.117 & 0.122 & 167 & 175 & 105 \\
\hline & \multicolumn{2}{|c|}{$0.35 \mu \mathrm{g} / \mathrm{mL}$} & 0.093 & 0.101 & 140 & 144 & 103 \\
\hline & \multicolumn{2}{|c|}{$0.25 \mu \mathrm{g} / \mathrm{mL}$} & 0.069 & 0.076 & 100 & 108 & \\
\hline \multirow[t]{14}{*}{ Linearity } & \multicolumn{7}{|c|}{ Spiked with recombinant active VWF (R1306W) } \\
\hline & \multicolumn{7}{|c|}{ Range assessed: OD $0.073-0.635(90.1 \%-787.2 \%$ of NPP) } \\
\hline & \multicolumn{7}{|c|}{ Linear range: OD $0.073-0.342(90.1 \%-424.5 \%$ of NPP) } \\
\hline & \multicolumn{7}{|c|}{ Linearity curve through points: $R^{2}=0.9923$} \\
\hline & Dilution & f low ac & ve VWF & I with high & ctive $V$ & F pool & \\
\hline & Range: $\mathrm{O}$ & $0.083-C$ & 199 & & & & \\
\hline & & & & VWF mean & SD) & $\%$ & ted \\
\hline & $100 \% \mathrm{H}$ & $0 \% \mathrm{~L}$ & & 5.5) & & - & \\
\hline & $75 \% \mathrm{H} /$ & $5 \% \mathrm{~L}$ & & 2.1) & & 10 & \\
\hline & $50 \% \mathrm{H} /$ & $0 \% \mathrm{~L}$ & & 1.9) & & 97 & \\
\hline & $25 \% \mathrm{H} /$ & $5 \% \mathrm{~L}$ & & 1.9) & & 10 & \\
\hline & $0 \% \mathrm{H} / 1$ & $0 \% \mathrm{~L}$ & & 2.3) & & - & \\
\hline & Linear fit & hrough & served & vs expected & OD po & $s: R^{2}=0.98$ & \\
\hline & $O D$ & $\%$ of & $\mathrm{VPP}$ & & & & \\
\hline LoQ & 0.081 & 101. & & & & & \\
\hline LoB & 0.060 & 80.0 & & & & & \\
\hline LoD & 0.067 & 89.1 & & & & & \\
\hline Stability & Baseline & ctive VY & $153 \%$ & $2.7 \%)$ & & & \\
\hline & Repeate & freeze- $t$ & awing & & Exten & storage & \\
\hline & & Reco & ery, me & & & Recovery, $n$ & \\
\hline & Thaw \# & $\begin{array}{l}\text { Absc } \\
(\% \mathrm{c}\end{array}$ & $\begin{array}{l}\text { ute } \\
\text { NPP) }\end{array}$ & $\begin{array}{l}\text { Rel. to BL } \\
\text { (\%) }\end{array}$ & $t$ & $\begin{array}{l}\text { Absolute } \\
\text { (\% of NPP) }\end{array}$ & $\begin{array}{l}\text { Rel. to BL } \\
\text { (\%) }\end{array}$ \\
\hline & 1 & 150 & 3.8) & 98 & $24 \mathrm{~h}$ & $153(2.6)$ & 100 \\
\hline & 2 & 146 & $3.2)$ & 95 & $48 \mathrm{~h}$ & $152(3.3)$ & 100 \\
\hline & 3 & 138 & 4.4) & 90 & $7 d$ & $150(4.0)$ & 98 \\
\hline & 4 & 129 & $3.2)$ & 84 & $1 \mathrm{~m}$ & $146(3.2)$ & 95 \\
\hline & & & & & $2 m$ & $147(2.3)$ & 96 \\
\hline
\end{tabular}

(Caption Table 1) SD, standard deviation; L, low; M, medium; H, high; E, expected; O, observed; OD, optical density; $[\mathrm{E}]_{\%} /[\mathrm{O}]_{\%}$, expected and observed active VWF level, as a \% of NPP; LoO, limit of quantitation; LoB, limit of blank; LoD, limit of detection; NPP, normal pooled plasma; Rel. to BL, relative to baseline value, calculated as $100 x$ (observed value/baseline), expressed as $\%$; $t$, timepoint; $h$, hours; d, days; m, month. 


\section{Analytical performance of the active VWF assay}

We proceeded with the analytical characterization of the assay, summarized in Table 1 and depicted in Figures 2B-F. Precision studies revealed intra-assay CVs of $2.4-7.2 \%$ and inter-assay CVs of 4.1-9.4\%, depending on measurand level (Figure 2B). Spiked R1306W VWF could be accurately measured in a plasma matrix at concentrations as low as $0.25 \mu \mathrm{g} / \mathrm{mL}$, with observed levels well within $90-110 \%$ of the expected levels (Figure $2 C)$. The assay was linear $\left(R^{2}>0.99\right)$ in almost the entire range of dilutions of plasma spiked with R1306W (Figure 2D and Table 1). Recovery of active VWF in mixes of low $(\mathrm{L})$ and high $(\mathrm{H})$ plasma pools was within the pre-defined acceptable limits of $90-110 \%$. Moreover, the curve fitted through the 5 points for these mixes (expected OD based on levels in individual pools versus observed) was linear $\left(R^{2}=0.99\right)$ (Figure $\left.2 \mathrm{E}\right)$. The limit of quantitation (LoO) was $101 \%$ (corresponding to an OD of 0.081). The limit of blank (LoB) and limit of detection (LoD) were $80.0 \%$ (OD 0.060) and 89.1\% (OD 0.067), respectively. Active VWF in citrated plasma was stable for at least 2 months (longest time tested) at $-80^{\circ} \mathrm{C}$ (Table 1 ). Importantly, freeze-thawing up to 3 times slightly decreased the detected active VWF, whereas more frequent freeze-thawing resulted in the loss of analyte exceeding the criterion of $10 \%$ (Figure $2 \mathrm{~F}$ ).

Table 2. Demographic and laboratory results of healthy donors in reference interval study.

\begin{tabular}{|c|c|c|c|c|c|}
\hline Parameter & \multicolumn{2}{|l|}{ All } & Male $(n=60)$ & Female $(n=60)$ & p-value \\
\hline \multicolumn{6}{|l|}{ General } \\
\hline Age (years) & \multicolumn{2}{|c|}{$31(25-44.5)$} & $29(25-39)$ & $32.5(26-50)$ & ns \\
\hline OC use (\%) & \multicolumn{2}{|l|}{15} & - & $15(25 \%)$ & - \\
\hline \multicolumn{6}{|l|}{ Haematological } \\
\hline \multirow[t]{4}{*}{ Blood group ${ }^{a}(\%)$} & 0 & $n=26(40 \%)$ & $n=12(41 \%)$ & $\mathrm{n}=14(39 \%)$ & ns \\
\hline & A & $n=29(45 \%)$ & $n=12(41 \%)$ & $n=17(47 \%)$ & \\
\hline & B & $n=9(14 \%)$ & $n=5(17 \%)$ & $n=4(11 \%)$ & \\
\hline & $A B$ & $n=1(1 \%)$ & & $n=1(3 \%)$ & \\
\hline Platelets ( $\left.10^{9} / \mathrm{L}\right)$ & \multicolumn{2}{|c|}{$233(208-270)$} & $229(207-257)$ & $244(216-284)$ & ns \\
\hline WBC ( $\left.\times 10^{9} / \mathrm{L}\right)$ & \multicolumn{2}{|c|}{$5.2(4.6-6.0)$} & $5.2(4.6-5.8)$ & $5.3(4.7-6.2)$ & ns \\
\hline $\mathrm{RBC}\left(\times 10^{12} / \mathrm{L}\right)$ & \multicolumn{2}{|c|}{$4.5(4.2-4.7)$} & $4.6(4.5-4.8)$ & $4.3(4.1-4.5)$ & $<0.0001$ \\
\hline $\mathrm{Hb}(\mathrm{mmol} / \mathrm{L})$ & \multicolumn{2}{|c|}{$7.9(7.5-8.4)$} & $8.3(8.0-8.6)$ & $7.6(7.2-7.9)$ & $<0.0001$ \\
\hline Hct (L/L) & \multicolumn{2}{|c|}{$0.39(0.37-0.41)$} & $0.40(0.39-0.41)$ & $0.37(0.35-0.39)$ & $<0.0001$ \\
\hline MPV (fL) & \multicolumn{2}{|c|}{$7.5(7.1-8.2)$} & $7.4(7.1-8.2)$ & $7.6(7.2-8.3)$ & ns \\
\hline
\end{tabular}

$O C$, oral contraceptives; freq, frequency; WBC, white blood cell count; $R B C$, red blood cell count; $\mathrm{Hb}$, haemoglobin; Hct, haematocrit; MPV, mean platelet volume. ${ }^{a}$ Blood group known for 65 individuals (36 female, 29 male), percentages indicate proportions for each blood group from total individuals with known blood group. Medians and interquartile ranges $(25-75 \%)$ are given unless otherwise indicated. 


\section{Reference interval and inter-individual variation for (active) VWF}

Demographic data and blood counts of 120 healthy individuals are summarized in Table 2. Haemoglobin levels, haematocrit and red blood cell counts were significantly higher in male compared to female subjects. The distribution of active VWF (Figure S1) in this population showed a clear right-skewedness, with the majority of values approximating that in NPP (100\%). The corresponding reference interval for active VWF was 91.6154.8\%. Reference intervals for VWF:Ag, VWF:RCo, VWF:GP1bM, VWFpp and Plt:VWF binding were $65.1-189.9 \%, 47.0-161.5 \%, 58.3-189.7 \%, 73.3-205 \%$ and $1.4-15.6 \%$ respectively (Table 3 ). Moreover, active VWF had a considerably lower inter-individual variation (15.1\%) than all other VWF parameters.

\section{Mutual correlations of VWF parameters}

A variety of assays may be performed for the assessment of VWF in plasma, each quantifying a different form of VWF (globular native VWF:Ag, active VWF, VWFpp) or providing qualitative information on its function (VWF:RCo, VWF:GP1bM, Plt:VWF binding). Significant correlations were observed between all these VWF parameters in our study population. Active VWF levels were significantly and positively correlated with VWFpp ( $r=0.281, p=0.005)$, Plt:VWF binding $(r=0.273, p=0.003), V W F: A g$ levels $(r=0.390, p<0.001), V W F: R C o(r=0.401, p<0.001)$ and the highest correlation was found with VWF:GP1bM ( $r=0.464, p<0.001$ ) (Figure 3 and Table S1). Overall, the highest degree of correlation ( $p<0.001$ ) existed between VWF:GP1bM activity and VWF-platelet binding as measured by the Plt:VWF binding assay. There were also strong associations between these assays and the other two functional assays for active VWF and VWF:RCo, and between the functional assays and VWF:Ag (Table S1).

Table 3. Reference intervals for active VWF and other VWF parameters in $\mathbf{1 2 0}$ healthy individuals.

\begin{tabular}{lllllllll}
\hline Parameter & Mean & SD & \%CV & Median & IQR & $\begin{array}{l}\text { Ref. interval } \\
\mathbf{2 . 5 \% - 9 7 . 5 \%}\end{array}$ & Min & Max \\
\hline VWF:Act (\%) & 115.4 & 17.4 & 15.1 & 112.9 & 25.1 & $91.6-154.8$ & 91.0 & 167.9 \\
VWF:Ag (\%) & 115.1 & 32.2 & 28.0 & 104.5 & 39.8 & $65.1-189.9$ & 40.0 & 218.0 \\
VWF:RCo (\%) & 103.9 & 32.9 & 31.7 & 102.6 & 48.1 & $47.0-161.5$ & 20.8 & 243.4 \\
VWF:GP1bM (\%) & 112.9 & 32.9 & 29.1 & 110.6 & 43.1 & $58.3-189.7$ & 41.3 & 229.5 \\
VWFpp (\%) & 121.8 & 23.1 & 24.1 & 121.2 & 32.1 & $73.3-188.7$ & 60.3 & 210.3 \\
Plt:VWF (\%) & 6.9 & 3.2 & 46.4 & 6.4 & 3.8 & $1.4-15.6$ & 0.9 & 18.0 \\
\hline
\end{tabular}

$\% \mathrm{CV}$, inter-individual variation expressed as coefficient of variation, calculated as (SD/Mean)*100; $I Q R$, interquartile range, calculated as $75^{\text {th }}-25^{\text {th }}$ percentile; Ref. interval, reference interval, calculated as the $2.5^{\text {th }}$ percentile to the $97.5^{\text {th }}$ percentile of the distribution; VWF:Act, active VWF, \% of level in NPP. VWF:Ag, VWF antigen; VWF:RCo, VWF ristocetin cofactor activity; VWF:GP1bM, VWF binding to gain-of-function GP1b fragments; VWFpp, VWF propeptide, \% of level in NPP; Plt:VWF, platelet-VWF binding, \% of signal for beads. 

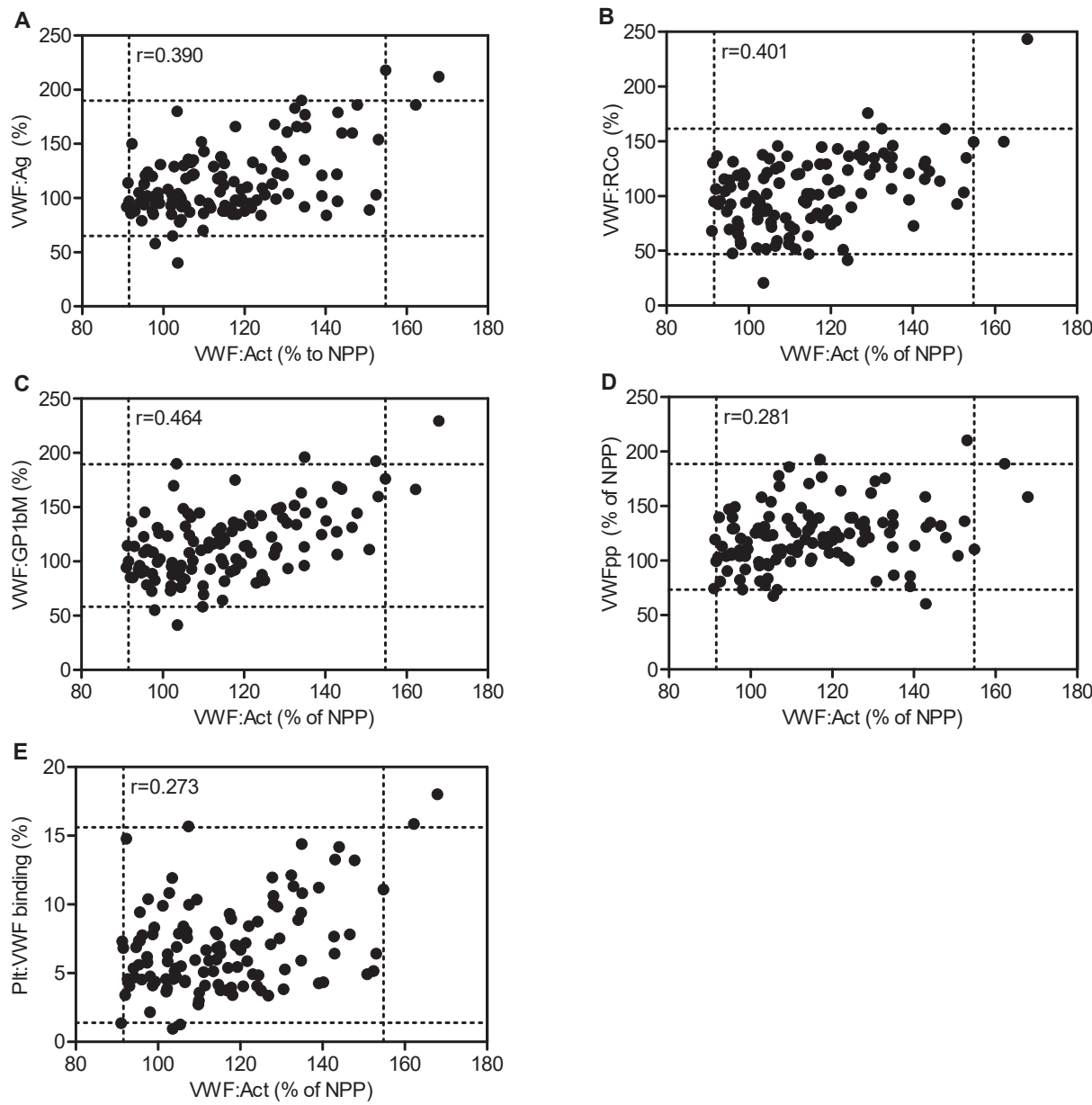

Figure 3. Correlation of active VWF with other VWF parameters. Scatterplots and corresponding Spearman rank correlations ( $r$ ) between active VWF assay (VWF:Act) and (A) VWF:Ag, (B) VWF:RCo, (C) VWF:GP1bM, (D) VWFpp and (E) Plt:VWF binding. Dotted lines delineate reference intervals determined in this study, with the centre square containing $95 \%$ of values. Spearman rank correlation coefficients $(r)$ are indicated in the upper left corner of each panel.

\section{VWF parameters related to clinical parameters}

Active VWF levels correlated with gender $(r=0.21, p=0.019)$ and were significantly ( $p=0.033$ ) higher in male (median $115.2 \%$, IOR 105.6-127.4\%) compared to female subjects (107.1\%, IOR 97.5 - 126.1\%). Therefore, sex-specific reference intervals were calculated, but these did not differ considerably (93.3-158.5\% for men, $91.2-159.7 \%$ for women), and corresponding confidence intervals overlapped (Figure S2). VWF:Ag levels were also significantly $(\mathrm{p}=0.044)$ higher in male (median $112.5 \%$, IOQR 95.5$135.3 \%$ ) compared to female subjects (median 99.5\%, IOR 91.0-122.5\%). Adjustment 
for VWF:Ag levels using multiple linear regression strongly reduced the association between active VWF and sex $(p=0.047$ to $p=0.289)$. Active VWF did not correlate with age, whereas significant positive correlations of VWF:Ag levels $(r=0.236, p=0.009)$ and VWF:RCo $(r=0.181, p=0.048)$ with age were observed (Figure S3). No association between active VWF levels and blood group was found ( $r=0.196, p=0.118)$, while VWF:Ag levels $(r=0.296, p=0.018), V W F: R C o(r=0.299, p=0.017), V W F: G P 1 B m(r=0.280, p=0.025)$ and Plt:VWF binding $(r=0.293, p=0.021)$ were significantly lower in 0 compared to non-O blood group subjects (Figure S4). However, in multiple linear regression analysis adjustment for VWF:Ag levels eliminated the significant association between VWF:RCo $(p=0.270), V W F: G P 1 b M(p=0.297)$ and Plt:VWF binding $(p=0.612)$ with blood group.

\section{DISCUSSION}

Here, we describe an immunosorbent assay for the reliable, accurate and specific quantification of active VWF in citrated plasma. We established its reference interval in a healthy population and we demonstrated a clear association of active VWF levels with four clinically established VWF assays and a flow cytometric VWF-platelet binding assay.

The active VWF assay fulfilled our pre-defined analytical requirements. First, the assay was highly specific for active VWF, as only the two recombinant VWF proteins that are constitutively in their active conformation were detected and no cross-reactivity with native (NPP) and purified HVWF was observed. This is in accordance with findings by Hulstein et al. ${ }^{13}$ Of note, serial dilutions of NPP did not substantially increase the OD since NPP only contains minute amounts of active VWF. Reproducibility was adequate, with values below $10 \%$ for both the intra- and inter-assay CV, comparable with those found by others. ${ }^{13,18}$ All values obtained from the population of healthy individuals were above the LoD (89.1\%), whereas $26(22 \%)$ values were below the LoO of $101 \%$. This is not necessarily a limitation, since high active VWF levels are much more relevant as they may induce thrombosis or bleeding. Lastly, active VWF showed good stability in samples stored at $-80^{\circ} \mathrm{C}$, and a maximum of 3 freeze-thawing steps should be allowed to prevent analyte loss of more than $10 \%$. This effect of freeze-thawing may be explained by an increased proportion of small molecular weight fragments, as was observed previously. ${ }^{31}$ Taken together, these data demonstrate the robust analytical performance of this assay.

In order to identify elevated active VWF levels in patient samples, it is essential to establish a reference interval in healthy individuals. To this end, we determined ac- 
tive VWF levels in 120 healthy individuals, as recommended by CLSI guidelines. ${ }^{30}$ The reference range shows a right-skewed distribution, with most individuals having very low active VWF levels, similar to that in NPP (set to 100\%). The right skew indicates that slightly more active VWF is present in native plasma of some healthy individuals, and these small differences could be detected due to the high sensitivity of our assay. The reference interval includes the majority of active VWF levels reported by others, although values below the LoD of the current assay were previously reported. ${ }^{16,20,21,32}$ This difference may be attributed to an alternative calculation method for the VWF 'activation factor' (see limitations below). ${ }^{13}$ Of note, the number of healthy controls in previous studies was much lower than 120 , ranging from nine ${ }^{13,14}$ to fifty-nine. ${ }^{17}$ In addition, we determined VWF:Ag, VWF:RCo, VWF:GP1bM, VWFpp and Plt:VWF binding in these 120 samples.

Increased active VWF can potentially have major impact on the risk of bleeding and/ or thrombosis, as exemplified by VWD type $2 \mathrm{~B}$ and TTP. ${ }^{13}$ Therefore, regulatory mechanisms, for instance the natural inhibitor $\beta 2 \mathrm{GPI}$, are keeping active VWF levels low in healthy individuals. ${ }^{16}$ The inter-individual variation in active VWF levels (15.1\%) was nearly half of that in VWF:Ag levels and the majority of active VWF values were close to the level in NPP (100\%). The observed inter-individual variation for VWF:Ag was similar to the previously reported inter-individual biologic variation for $\mathrm{VWF}^{33}$

We identified significant positive correlations between all VWF parameters (Table S1). With regard to active VWF, the highest correlation was found with two other activity assays, namely VWF:RCo and VWF:GP1bM as expected based on assay principles. Active VWF circulates in a GPIb $\alpha$-binding conformation and will therefore contribute to the chemiluminescent signal in the VWF:RCo assay, generated when VWF (either active VWF already present or VWF activated by ristocetin) binds to microparticles coated with GPIb $\alpha$. Similarly, in the VWF:GP1bM assay only active VWF can bind spontaneously to the microparticle-(mutant) GP1b complex, inducing agglutination that is measured turbidimetrically. Whereas the VWF:GP1bM and VWF:RCo assays are much more widely established, advantages of the active VWF ELISA assay are that neither non-physiological additives (ristocetin or mutant GP1b) nor specialized instruments are required. Moreover, active VWF correlated well with VWF:Ag levels $(r=0.390)$, showing that with increasing VWF the amount of circulating VWF in its active, unfolded conformation was also higher. This is in accordance with correlations found between VWF activation factor and VWF:Ag in previous studies in patients with a first STEMI $(r=0.58)^{18}$ and malignant hypertension $(r=0.62) .{ }^{34}$ The stronger correlation in these studies may be explained by reporting the activation factor, which corrects for differences in VWF:Ag levels. Moreover, active VWF correlated with VWFpp. Relatively increased VWFpp is commonly used 
as a marker for acute endothelial cell activation, while relatively high VWF:Ag levels are considered a marker for more chronic endothelial cell activation. ${ }^{27}$ VWF in endothelial cells is likely in its active conformation, as freshly secreted VWF is able to interact with the platelet Gplb-IX-V complex. ${ }^{5}$ Thus, it can be postulated that circulating active VWF was secreted from endothelial cells (hence correlating with VWFpp and VWF:Ag levels), but escaped ADAMTS-13 proteolysis.

In our healthy population, active VWF and VWF:Ag levels were significantly higher in men than in women In contrast to the activation factor calculation method ${ }^{13}$, our calculation of active VWF levels does not correct for differences in VWF:Ag levels. Therefore, sex-related differences in active VWF levels have not been reported in previous studies. Whereas the majority of the literature on the influence of sex (gender) on VWF:Ag reports no differences ${ }^{9,35}$, Conlan et al. reported higher VWF:Ag levels in female compared to male subjects ${ }^{36}$, while Campos et al. found higher VWF:Ag levels in men than in women. ${ }^{37}$ In addition, both older age and non-O blood group were associated with higher VWF:Ag levels, as described in literature.9. 38-41 VWF:RCo, VWF:GP1bM and Plt:VWF binding were also higher in individuals with a non-O blood group, but correction for VWF:Ag levels rendered these associations non-significant.

A possible limitation of the current assay is that it expresses the amount of active VWF as a percentage, normalized to values in NPP. We consider this a more reliable approach than expressing the absolute concentration with regard to a monomeric VWF A1 domain, because one VHH binds active VWF multimers with different sizes..$^{42}$ and hence variable numbers of VWF epitopes. The assay cannot adjust for variation in the multimeric composition between individuals. In addition, in order to calculate the concentration of active VWF, we would need to assume that the $\mathrm{VHH}$ recognizes mutation-induced activated VWF (e.g. R1306W) similarly as non-mutated (activated) VWF and as active VWF in a variety of conditions. Because we are uncertain of these assumptions, we propose that expressing active VWF as a percentage of NPP is a more reliable calibrator for the moment. However, the normalized result makes it more difficult to standardize the assay, and the reported reference interval may have been slightly different using another batch of NPP. Given the small inter-individual variation in active VWF in healthy individuals and the large increases observed in several pathologies ${ }^{5}$, we do not expect minor differences in NPP active VWF levels to cause the wrong classification of active VWF in a sample as pathological. 


\section{CONCLUSIONS}

Previously, it has been shown that several pathological conditions are associated with strongly increased active VWF levels. ${ }^{5}$ The underlying mechanism can likely be attributed to (a combination of) three causes: (1) changes in the (conformation of) VWF itself (e.g. VWD type $2 \mathrm{~B}^{13}$ ), (2) changes in endothelial secretion (e.g. malaria ${ }^{20}$, diabetes ${ }^{17}$ ), processing or clearance of VWF (e.g. TTP ${ }^{13}$, HELLP $^{14}$ ) or (3) increased shear stress (e.g. mild aortic stenosis ${ }^{32}$, first STEMI ${ }^{18}$ ). Increased circulating active VWF levels may also be present in other conditions associated with infection and/or inflammation, for instance in chronic kidney disease. ${ }^{43}$ Moreover, using the current assay, we found that active VWF levels in healthy individuals increase following strenuous exercise. ${ }^{44}$ Quantification of active VWF in a variety of diseases could provide valuable pathophysiological insight for diagnosis and development of new treatment strategies. The current validation of an immunosorbent assay for active VWF and the establishment of a reference interval will facilitate future studies investigating the role of active VWF in health and disease.

\section{ACKNOWLEDGEMENTS}

We would like to thank V. Verschuur, G. Oelemans-Prins, W. Gerritsen, M. KoldenhofVisser, I. Bakker-Wolbers and B. Wildeboer (Gelre Hospitals Apeldoorn) for assistance with the VWF:Ag and VWF:RCo measurements and M. van Wijnen, M. Siderius and B. Blankenspoor (Meander Medical Center Amersfoort) for their help with the VWF:GP1bM measurements. We are grateful to our colleagues at Synapse Research Institute Maastricht for recruiting donors and for blood drawing and processing. 


\section{REFERENCES}

1. Lenting PJ, Christophe OD, Denis CV. von Willebrand factor biosynthesis, secretion, and clearance: connecting the far ends. Blood. 2015;125(13):2019-28.

2. Savage B, Saldivar E, Ruggeri ZM. Initiation of platelet adhesion by arrest onto fibrinogen or translocation on von Willebrand factor. Cell. 1996;84(2):289-97.

3. Springer TA. von Willebrand factor, Jedi knight of the bloodstream. Blood. 2014;124(9):141225.

4. Huizinga EG, Tsuji S, Romijn RA, Schiphorst ME, de Groot PG, Sixma JJ, et al. Structures of glycoprotein Ibalpha and its complex with von Willebrand factor A1 domain. Science. 2002;297(5584):1176-9.

5. Groot E, de Groot PG, Fijnheer R, Lenting PJ. The presence of active von Willebrand factor under various pathological conditions. Curr Opin Hematol. 2007;14(3):284-9.

6. Ruggeri ZM, Zimmerman TS. von Willebrand factor and von Willebrand disease. Blood. 1987;70(4):895-904.

7. Sharma R, Flood VH. Advances in the diagnosis and treatment of Von Willebrand disease. Hematology Am Soc Hematol Educ Program. 2017;2017(1):379-84.

8. Sadler JE. Von Willebrand factor, ADAMTS13, and thrombotic thrombocytopenic purpura. Blood. 2008;112(1):11-8.

9. Favaloro EJ, Soltani S, McDonald J, Grezchnik E, Easton L, Favaloro JW. Reassessment of ABO blood group, sex, and age on laboratory parameters used to diagnose von Willebrand disorder: potential influence on the diagnosis vs the potential association with risk of thrombosis. Am J Clin Pathol. 2005;124(6):910-7.

10. Lippi G, Franchini M, Salvagno GL, Montagnana M, Poli G, Guidi GC. Correlation between von Willebrand factor antigen, von Willebrand factor ristocetin cofactor activity and factor VIII activity in plasma. J Thromb Thrombolysis. 2008;26(2):150-3.

11. Patzke J, Favaloro EJ. Laboratory Testing for von Willebrand Factor Activity by Glycoprotein Ib Binding Assays (VWF:GPIb). Methods Mol Biol. 2017;1646:453-60.

12. Patzke J, Budde U, Huber A, Mendez A, Muth H, Obser T, et al. Performance evaluation and multicentre study of a von Willebrand factor activity assay based on GPIb binding in the absence of ristocetin. Blood Coagul Fibrinolysis. 2014;25(8):860-70.

13. Hulstein JJ, de Groot PG, Silence K, Veyradier A, Fijnheer R, Lenting PJ. A novel nanobody that detects the gain-of-function phenotype of von Willebrand factor in ADAMTS13 deficiency and von Willebrand disease type 2B. Blood. 2005;106(9):3035-42.

14. Hulstein JJ, van Runnard Heimel PJ, Franx A, Lenting PJ, Bruinse HW, Silence K, et al. Acute activation of the endothelium results in increased levels of active von Willebrand factor in hemolysis, elevated liver enzymes and low platelets (HELLP) syndrome. J Thromb Haemost. 2006;4(12):2569-75.

15. Hyseni A, Kemperman H, de Lange DW, Kesecioglu J, de Groot PG, Roest M. Active von Willebrand factor predicts 28-day mortality in patients with systemic inflammatory response syndrome. Blood. 2014;123(14):2153-6.

16. Hulstein JJ, Lenting PJ, de Laat B, Derksen RH, Fijnheer R, de Groot PG. beta2-Glycoprotein I inhibits von Willebrand factor dependent platelet adhesion and aggregation. Blood. 2007;110(5):1483-91. 
17. Chen SF, Xia ZL, Han JJ, Wang YT, Wang JY, Pan SD, et al. Increased active von Willebrand factor during disease development in the aging diabetic patient population. Age (Dordr). 2013;35(1):171-7.

18. Rutten B, Maseri A, Cianflone D, Laricchia A, Cristell NA, Durante A, et al. Plasma levels of active Von Willebrand factor are increased in patients with first ST-segment elevation myocardial infarction: a multicenter and multiethnic study. Eur Heart J Acute Cardiovasc Care. 2015;4(1):64-74.

19. Chen J, Hobbs WE, Le J, Lenting PJ, de Groot PG, Lopez JA. The rate of hemolysis in sickle cell disease correlates with the quantity of active von Willebrand factor in the plasma. Blood. 2011;117(13):3680-3.

20. de Mast $\mathrm{Q}$, Groot E, Lenting PJ, de Groot PG, McCall M, Sauerwein RW, et al. Thrombocytopenia and release of activated von Willebrand Factor during early Plasmodium falciparum malaria. J Infect Dis. 2007;196(4):622-8.

21. Djamiatun K, van der Ven AJ, de Groot PG, Faradz SM, Hapsari D, Dolmans WM, et al. Severe dengue is associated with consumption of von Willebrand factor and its cleaving enzyme ADAMTS-13. PLoS Negl Trop Dis. 2012;6(5):e1628.

22. Casonato A, Pontara E, Morpurgo M, Sartorello F, De Groot PG, Cattini MG, et al. Higher and lower active circulating VWF levels: different facets of von Willebrand disease. $\mathrm{Br}]$ Haematol. 2015;171(5):845-53.

23. van Asten I, Schutgens REG, Baaij M, Zandstra J, Roest M, Pasterkamp G, et al. Validation of flow cytometric analysis of platelet function in patients with a suspected platelet function defect. J Thromb Haemost. 2018;16(4):689-98.

24. Giannini S, Cecchetti L, Mezzasoma AM, Gresele P. Diagnosis of platelet-type von Willebrand disease by flow cytometry. Haematologica. 2010;95(6):1021-4.

25. Giannini S, Mezzasoma AM, Leone M, Gresele P. Laboratory diagnosis and monitoring of desmopressin treatment of von Willebrand's disease by flow cytometry. Haematologica. 2007;92(12):1647-54.

26. Lankhof H, Damas C, Schiphorst ME, MJ IJ, Bracke M, Sixma JJ, et al. Functional studies on platelet adhesion with recombinant von Willebrand factor type $2 \mathrm{~B}$ mutants R5430 and R543W under conditions of flow. Blood. 1997;89(8):2766-72.

27. van Mourik JA, Boertjes R, Huisveld IA, Fijnvandraat K, Pajkrt D, van Genderen PJ, et al. von Willebrand factor propeptide in vascular disorders: $A$ tool to distinguish between acute and chronic endothelial cell perturbation. Blood. 1999;94(1):179-85.

28. Huskens D, Sang Y, Konings J, van der Vorm L, de Laat B, Kelchtermans H, et al. Standardization and reference ranges for whole blood platelet function measurements using a flow cytometric platelet activation test. PLoS One. 2018;13(2):e0192079.

29. Crawley JT, de Groot R, Xiang Y, Luken BM, Lane DA. Unraveling the scissile bond: how ADAMTS13 recognizes and cleaves von Willebrand factor. Blood. 2011;118(12):3212-21.

30. CLSI and IFCC-Ad. Defining, establishing and verifying reference intervals in the clinical laboratory: approved guideline-third edition. 2008. p. 1-76.

31. Zimmerman TS, Dent JA, Ruggeri ZM, Nannini LH. Subunit composition of plasma von Willebrand factor. Cleavage is present in normal individuals, increased in IIA and IIB von Willebrand disease, but minimal in variants with aberrant structure of individual oligomers (types IIC, IID, and IIE). J Clin Invest. 1986;77(3):947-51. 
32. Hollestelle MJ, Loots CM, Squizzato A, Renne T, Bouma BJ, de Groot PG, et al. Decreased active von Willebrand factor level owing to shear stress in aortic stenosis patients. J Thromb Haemost. 2011;9(5):953-8.

33. Ricos C, Alvarez V, Cava F, Garcia-Lario JV, Hernandez A, Jimenez CV, et al. Desirable specifications for total error, imprecision, and bias, derived from intra- and inter-individual biologic variation. [updated 2014; cited 20186 July]. Available from: http://www.westgard.com/ biodatabase1.htm.

34. van den Born BJ, van der Hoeven NV, Groot E, Lenting PJ, Meijers JC, Levi M, et al. Association between thrombotic microangiopathy and reduced ADAMTS13 activity in malignant hypertension. Hypertension. 2008;51(4):862-6.

35. Zhou Z, Yu F, Buchanan A, Fu Y, Campos M, Wu KK, et al. Possible race and gender divergence in association of genetic variations with plasma von Willebrand factor: a study of ARIC and 1000 genome cohorts. PLoS One. 2014;9(1):e84810.

36. Conlan MG, Folsom AR, Finch A, Davis CE, Sorlie P, Marcucci G, et al. Associations of factor VIII and von Willebrand factor with age, race, sex, and risk factors for atherosclerosis. The Atherosclerosis Risk in Communities (ARIC) Study. Thromb Haemost. 1993;70(3):380-5.

37. Campos M, Sun W, Yu F, Barbalic M, Tang W, Chambless LE, et al. Genetic determinants of plasma von Willebrand factor antigen levels: a target gene SNP and haplotype analysis of ARIC cohort. Blood. 2011;117(19):5224-30.

38. Gallinaro L, Cattini MG, Sztukowska M, Padrini R, Sartorello F, Pontara E, et al. A shorter von Willebrand factor survival in $O$ blood group subjects explains how $A B O$ determinants influence plasma von Willebrand factor. Blood. 2008;111(7):3540-5.

39. Rydz N, Grabell J, Lillicrap D, James PD. Changes in von Willebrand factor level and von Willebrand activity with age in type 1 von Willebrand disease. Haemophilia. 2015;21(5):63641.

40. Moeller A, Weippert-Kretschmer M, Prinz H, Kretschmer V. Influence of ABO blood groups on primary hemostasis. Transfusion. 2001;41(1):56-60.

41. Albanez S, Ogiwara K, Michels A, Hopman W, Grabell J, James P, et al. Aging and ABO blood type influence von Willebrand factor and factor VIII levels through interrelated mechanisms. J Thromb Haemost. 2016;14(5):953-63.

42. Groot E, Fijnheer R, Sebastian SA, de Groot PG, Lenting PJ. The active conformation of von Willebrand factor in patients with thrombotic thrombocytopenic purpura in remission. J Thromb Haemost. 2009;7(6):962-9.

43. van der Vorm L, Visser R, Huskens D, Veninga A, Adams D, Remijn J, et al. Circulating active von Willebrand factor levels are increased in chronic kidney disease and end-stage renal disease. CKJ. 2019:1-3.

44. van der Vorm LN, Huskens D, Kicken CH, Remijn JA, Roest M, de Laat B, et al. Effects of Repeated Bouts of Exercise on the Hemostatic System. Semin Thromb Hemost. 2018;44(8):710-22. 


\section{SUPPLEMENTAL DATA}

\section{Supplemental Methods}

\section{Production of the $\mathrm{VHH}$}

VWF A1 domain-specific VHH antibodies (MW $16.4 \mathrm{kDa}$ ) were commercially produced by U-Protein Express BV (Utrecht, the Netherlands). Briefly, HEK293E-253 cells were transfected with endotoxin-free maxiprep DNA for the desired sequence of the antiVWF VHH (based on the sequence provided by ${ }^{1}$ ). Six days post-transfection conditioned medium containing recombinant protein was harvested by low-speed centrifugation (10 minutes, $1000 \mathrm{~g}$ ) followed by high-speed centrifugation (10 minutes, 4000g) and immobilized metal affinity chromatography (IMAC) purification. The VHH was further purified by gel filtration using a Superdex75 26/600 column. The resulting VHHcontaining fractions were sterilized by filtration over a $0.22 \mu \mathrm{m}$ syringe filter and stored at $4^{\circ} \mathrm{C}$.

\section{Assay performance studies}

\section{Specificity}

The assay was performed as described under "Active VWF immunosorbent assay" in the Methods section. Normal pooled plasma (NPP, static or vortexed at 2,500 rpm for 10 minutes), HVWF, R1306W VWF or R13060 VWF (all $1.7 \mu \mathrm{g} / \mathrm{mL}$ ), were serially diluted (except for vortexed NPP, only measured at 1:10 dilution) with dilution buffer (PBS/1\% BSA) and incubated in the plate coated with VHH at RT for 2 hours, followed by detection steps as described.

\section{Precision}

Pools of citrated plasma samples were prepared based on previously measured active VWF levels: the "low" (L) pool contained samples with active VWF levels between 91 and $110 \%$ (mean 102\%), the "medium" (M) pool contained samples with active VWF levels between 150 and 200\% (mean 182\%) and the "high" pool samples with active VWF levels $>220 \%$ (mean $235 \%$ ). These "cut-offs" were arbitrarily chosen, to cover the range of relevant values, and they do not have clinical meaning. Intra-assay (within run) precision was assessed by repeated $(n=20)$ measurements of these plasma pools active VWF in one plate on the same day. Inter-assay (between run) precision was determined by duplicate measurements of the same plasma pools on 20 different days, within 2 months. Acceptance criteria were CV $\leq 10 \%$ for intra-assay precision and CV $\leq 15 \%$ for inter-assay precision. 


\section{Accuracy}

Accuracy was assessed by adding known concentrations of recombinant R1306W VWF (stock $17 \mu \mathrm{g} / \mathrm{mL}$, concentrations $1.0,0.75,0.5,0.35$ or $0.25 \mu \mathrm{g} / \mathrm{mL}$ R1306W VWF) or dilution buffer (control) to NPP. Recovery (Rec\%) was calculated based on the observed $\mathrm{OD}\left(\mathrm{O}_{\mathrm{OD}}\right)$ and expected OD $\left(\mathrm{E}_{\mathrm{OD}}\right)$ as follows: $\operatorname{Rec} \%=\left(\mathrm{O}_{\mathrm{OD}} / \mathrm{E}_{\mathrm{OD}}\right) * 100 \%$ and was considered acceptable between $90 \%$ and $110 \%$.

\section{Linearity}

Linearity was determined by two serial recovery studies. First, for spiking with recombinant R1306W VWF, a pool of plasma with low active VWF was prepared. Half of this low active VWF pool was spiked with recombinant R1306W VWF (maximal $10 \mathrm{v} / \mathrm{v} \%$, not higher to minimize matrix dilution effects) to obtain a high active VWF pool. The high active VWF sample pool was then diluted with the remaining low active VWF sample pool to create a 12 step 1.2-fold dilution series. Secondly, to determine the linearity when diluting native plasma samples, a high pool (active VWF $>200 \%$ of NPP, as determined in previous experiments) was diluted with a low pool (active VWF $<120 \%$, as determined in previous experiments) in 5 steps: $100 \%$ of each and 3 mixes of $75 \% / 25 \%$, $50 \% / 50 \%$ and $25 \% / 75 \%$. For both dilution experiments, three replicates per dilution step were measured in 1 run.

\section{Limits}

The limit of quantitation (LoO) was determined using 11 citrated plasma pools over a range of concentrations (114-248\%), each measured in duplicate over 10 days. The LoQ was extrapolated from the binomial curve through the points in the plot of mean active VWF ( $x$-axis) versus inter-assay CV\% (y-axis) as the mean active VWF level at which the inter-assay CV was $20 \%$. The limit of blank (LoB) was determined by measuring a blank sample (dilution buffer) 60 -fold and calculated as $\mathrm{LoB}=$ mean $_{\text {blank }}+1.645 * \mathrm{SD}_{\text {blank }}$. The limit of detection (LoD) was determined based on the LoB and 60 test replicates of a sample known to contain a low concentration of the analyte (also used for intra-assay precision as "low/L" pool). LoD was calculated as $L o D=L o B+1.645\left(S_{\text {low sample }}\right)$.

\section{Stability}

We performed a stability study limited to the conditions used for (the validation of) this assay, i.e. with the aims to assess the (1) effect of repeated freeze/thawing and (2) stability during storage at $-80^{\circ} \mathrm{C}$. To determine freeze/thaw stability, a plasma pool with medium active VWF level (152.9\%) was stored frozen at $-80^{\circ} \mathrm{C}$ and was measured in triplicate with the immunosorbent assay after being subjected to four freeze-thaw cycles during one day. Furthermore, on day 1, day 2, day 7, 1 month and 2 months aliquots of this pool were thawed and active VWF was determined in triplicate. Mean 
recovery of active VWF expressed in absolute values (i.e., absolute recovery, expressed as active VWF in \% of NPP) and in percent of the baseline value (i.e., relative recovery) was calculated. The acceptance criterion was $\pm 10 \%$ difference from the original concentration.

\section{Flow cytometric analysis of VWF to platelet binding}

Test strips for three test conditions (no agonist control, $1.2 \mathrm{mg} / \mathrm{mL}$ ristocetin and positive beads control $[\mathrm{PC}]$ ) were prepared in advance and stored at $-20^{\circ} \mathrm{C}$. The reaction mixtures with a total volume of $20 \mu \mathrm{l}$ consisted of $2 \mu \mathrm{l} \mathrm{FITC-conjugated} \mathrm{anti-VWF} \mathrm{antibodies}$ (CL7616F, Cedarlane, Burlington, USA) and $0.5 \mu \mathrm{APC}$-conjugated anti-CD41a antibodies (559777, BD Biosciences, San Jose, USA) with or without agonist in HEPES-buffered saline (HBS, $10 \mathrm{mmol} / \mathrm{L}$ HEPES, $150 \mathrm{mmol} / \mathrm{L} \mathrm{NaCl}, 1 \mathrm{mmol} / \mathrm{L} \mathrm{MgSO}{ }_{4} 5 \mathrm{mmol} / \mathrm{L} \mathrm{KCL}, \mathrm{pH}$ 7.4). The PC tube only contained $2 \mu \mathrm{l}$ FITC-conjugated anti-VWF antibodies in HBS.

After venipuncture, whole blood was kept at RT for at least 30 minutes and subsequently incubated at $37^{\circ} \mathrm{C}$ for 10 minutes. All tests were performed at $37^{\circ} \mathrm{C}$. Blood was diluted $1: 4$ in HBS. From the diluted blood, $5 \mu \mathrm{l}$ was added to the tubes with reaction mixture ( $20 \mu \mathrm{l}$, final dilution 1:20). In addition, $5 \mu \mathrm{l}$ of anti-mouse $\lg \kappa$ particles was added to the PC tube. Tests were incubated for exactly 20 minutes at $37^{\circ} \mathrm{C}$. Reactions were stopped by adding $250 \mu \mathrm{l}$ fixation solution $(137 \mathrm{mmol} / \mathrm{L} \mathrm{NaCl}, 2.7 \mathrm{mmol} / \mathrm{L} \mathrm{KCl}, 1.12 \mathrm{mmol} / \mathrm{L}$ $\mathrm{NaH}_{2} \mathrm{PO}_{4} 1.15 \mathrm{mmol} / \mathrm{L} \mathrm{KH_{2 }} \mathrm{PO}_{4}, 10.2 \mathrm{mmol} / \mathrm{L} \mathrm{Na}_{2} \mathrm{HPO}_{4}, 4 \mathrm{mmol} / \mathrm{L}$ EDTA, 0.5\% formaldehyde). Samples were analysed 1 day after the experiment. Flow cytometry was used to discriminate platelets from other cells, using the forward and side scatter pattern and by gating on the CD41a positive cells. Median fluorescent intensity in the FITC gate was selected to determine VWF-platelet binding. Results are corrected for the MFI in the unstimulated test condition and expressed as a \% of the MFI for the PC beads. 


\section{Supplemental Figures}
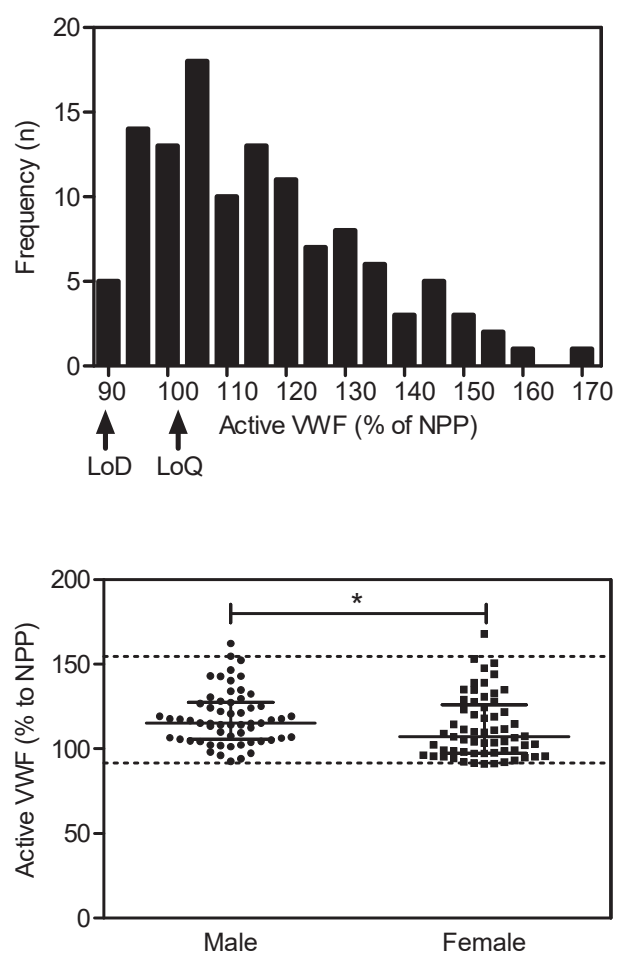

Figure S1. Distribution of active VWF in a healthy population. Citrated plasma samples obtained from 120 healthy subjects were measured with the active VWF assay. Limit of detection (LoD) and Limit of Quantitation (LoO) are indicated. The frequency distribution shows skewness to the right.
Figure S2. Effect of gender on active VWF levels. Active VWF levels (normalized to levels in NPP) were determined in plasma of 120 healthy volunteers, and are shown here for males $(n=60)$ and females $(n=60)$. Median and IOR are indicated. The area delineated by the dotted lines represents the reference interval of the total population (2.5 percentile-97.5 percentile). Active VWF levels were significantly $(p=0.033)$ higher in males compared to females, but adjustment for VWF:Ag levels abolished the association between active VWF and gender. 

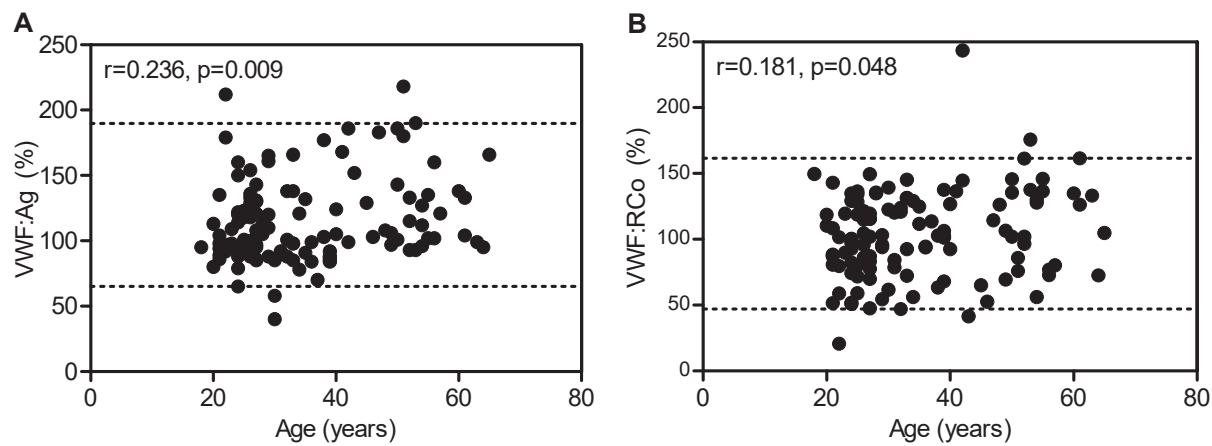

Figure S3. VWF:Ag and VWF:RCo correlate significantly with age. Scatterplot and corresponding Spearman rank correlation ( $r$ ) between VWF:Ag (a) and VWF:RCo (b) with age. Dotted lines delineate reference intervals for VWF:Ag and VWF:RCo determined in this study.
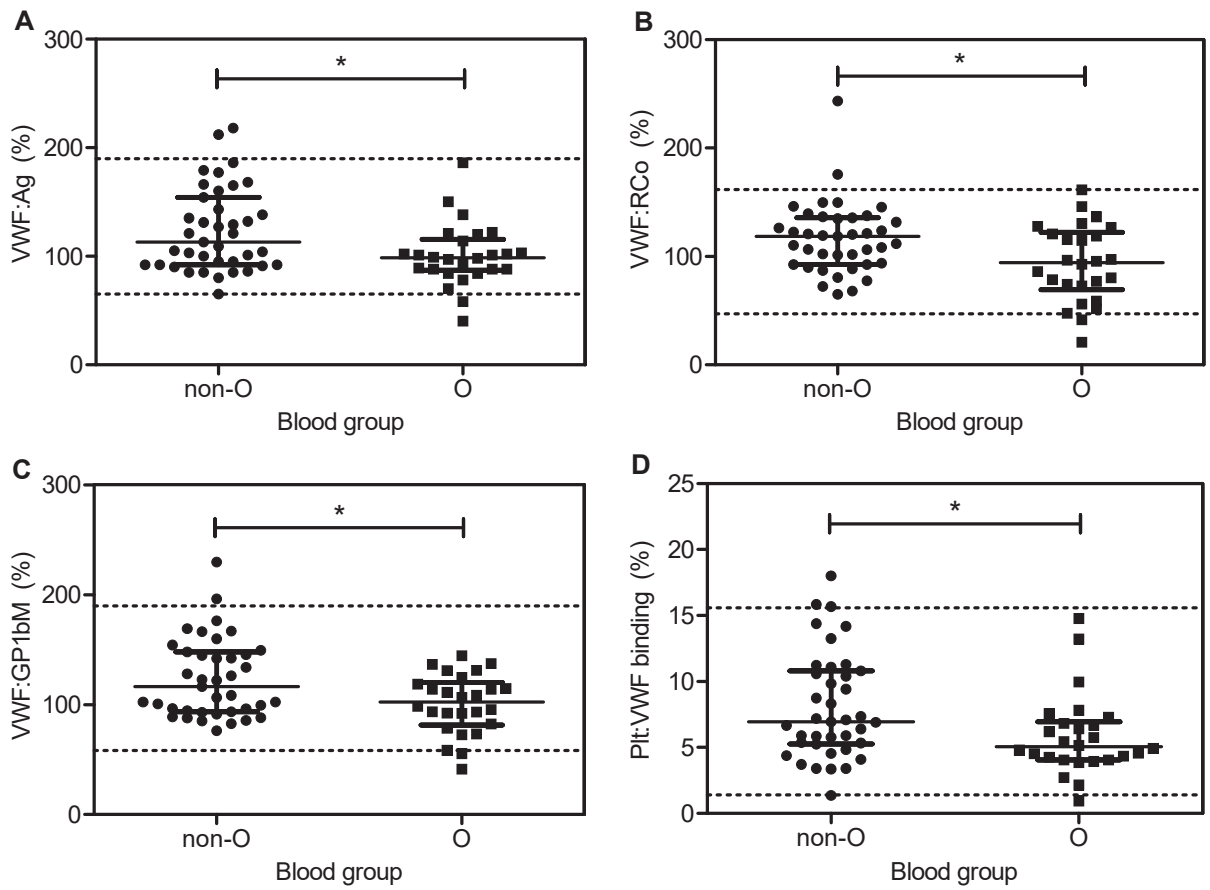

Figure S4. Effect of blood group 0 and non-0 on VWF parameters. VWF:Ag (A), VWF:RCo (B), VWF:GP1bM (C) and Plt:VWF binding (D) were determined in plasma of 120 healthy volunteers, and are shown here for individuals with non- $\mathrm{O}$ and $\mathrm{O}$ blood group. Median and IOR are indicated. The areas delineated by the dotted lines represent the reference intervals ( 2.5 percentile- 97.5 percentile). Statistical significance of differences in VWF parameters between 0 and non- 0 subjects were tested by Mann-Whitney U test. *, $p<0.05$. 


\section{Supplemental Tables}

S1 Table. Spearman rank correlations between VWF assays

\begin{tabular}{|c|c|c|c|c|c|c|}
\hline Assay & VWF:Act & VWF:Ag & VWF:RCo & VWF:GP1bM & VWFpp & Plt:VWF \\
\hline \multicolumn{7}{|l|}{ VWF:Act } \\
\hline VWF:Ag & 0.390 & & & & & \\
\hline VWF:RCo & 0.401 & 0.617 & & & & \\
\hline VWF:GP1bM & $0.464 *$ & $0.669 *$ & $0.564 *$ & & & \\
\hline VWFpp & $0.281^{* *}$ & $0.457^{* *}$ & $0.442^{* *}$ & $0.404^{* *}$ & & \\
\hline Plt:VWF & $0.273^{*}$ & 0.636 & $0.619 *$ & $0.679 *$ & 0.396 & \\
\hline
\end{tabular}

Values represent Spearman rank correlation coefficients $(r)$ with corresponding significance: $* *, p$ value <0.01. VWF:Act, active VWF; VWF:Ag, VWF antigen; VWF:RCo, VWF ristocetin cofactor activity; VWF:GP1bM, VWF binding to gain-of-function GP1b fragments; VWFpp, VWF propeptide; Plt:VWF, platelet VWF binding.

\section{REFERENCES}

1. Hulstein J], de Groot PG, Silence K, Veyradier A, Fijnheer R, Lenting PJ. A novel nanobody that detects the gain-of-function phenotype of von Willebrand factor in ADAMTS13 deficiency and von Willebrand disease type 2B. Blood. 2005;106(9):3035-42. 
Part II 
The effect of (repeated) strenuous exercise on active VWF 


$$
\text { Chapter } 4
$$




\section{Effects of repeated bouts of exercise on the haemostatic system}

Van der Vorm LN, Huskens D, Kicken $\mathrm{CH}$, Remijn JA, Roest $M$, de Laat $B$, Miszta $A$ 


\section{ABSTRACT}

Physical activity is beneficial for health, for example by lowering the risk of cardiovascular events. However, vigorous exercise is associated with the occurrence of thromboembolic events and sudden cardiac death, in particular in untrained individuals. Whereas acute exercise is known to cause a hypercoagulable state, repeated exposure to (strenuous) exercise by means of training may actually condition the haemostatic response to exercise. To date, the effects of exercise training on blood coagulability and the underlying mechanisms have yet to be fully discerned.

In the current review, we provide an overview of existing literature on how training programs and training status influence haemostasis in healthy individuals. Furthermore, we present data of a pilot study in which we studied the effects of repetitive submaximal intensity cycling on procoagulant and anticoagulant processes.

It is known that factor VIII and VWF increase after exercise, but we found that this increase in FVIII and VWF (antigen, propeptide and VWF in active conformation) was smaller on each of three subsequent days, suggesting either adaptation of endothelial activation or exhaustion of endothelial VWF supplies. With respect to thrombin generation, elevated FVIII significantly increased the thrombin generation peak but not the endogenous thrombin potential. In contrast, platelet activation in terms of P-selectin expression after stimulation with protease-activated receptor (PAR)-1 and glycoprotein (GP)VI agonists decreased after exercise and did not recover, indicating exhaustion of the platelet response to repetitive exercise. 


\section{INTRODUCTION}

Cardiovascular disease remains the leading cause of mortality and morbidity globally. ${ }^{1}$ Regular exercise is essential for a healthy lifestyle, promoting cardiovascular health, as shown by many epidemiological and clinical studies. ${ }^{2-4}$ Several studies have reported an association of increasing physical activity with reduced morbidity and mortality ${ }^{5,6}$, which can be explained by benefits such as weight loss, lowered blood pressure, and improved lipid profiles and glucose metabolism. ${ }^{7-9}$

Regular physical activity is associated with the prevention of cardiovascular disease, with an effect similar in magnitude $(-30 \%)$ to pharmacological strategies. ${ }^{10}$ However, more physical exercise may not always be better. Indeed, the benefit of exercise is still a subject of debate that is referred to as the "exercise paradox". ${ }^{11,12}$ The debate is relevant because there are numerous reports of exercise-related thromboembolic events, such as myocardial infarction, ischaemic stroke and venous thrombosis ${ }^{13},{ }^{14}$, and acute exercise is associated with a 2-3 fold higher risk of sudden cardiac death. ${ }^{15,16}$

One crucial protagonist of atherosclerotic and atherothrombotic disease is the haemostatic system. ${ }^{17}$ Exercise is known to exert a plethora of effects on haemostasis. These include increased levels of clotting factors (FVIII and von Willebrand factor (VWF)), platelet count and -reactivity, a shortening of the activated partial thromboplastin time $(\mathrm{aPTT})^{18,19}$ and increased thrombin generation (TG), ${ }^{20}$ as well as increases in fibrinolytic markers (prothrombin fragments $1+2$ and thrombin-antithrombin (TAT) complexes). ${ }^{21,22}$ Altogether, these changes result in a shift towards a transient hypercoagulable state ${ }^{23}$, which depends at least partly on exercise intensity ${ }^{24}$. Whereas moderate exercise enhances fibrinolytic activity, strenuous exercise (corresponding to $80-100 \%$ of the maximal heart rate) induces a more procoagulant state, causing an enhanced risk of thrombotic events, especially in untrained individuals. ${ }^{25-27}$ Contrary to acute highintensity exercise, regular training at moderate intensity is associated with a lower overall risk of adverse cardiovascular events. ${ }^{28,29}$

The effects of (strenuous) exercise on haemostasis have been reviewed extensively by others. ${ }^{23,26,30}$ Many studies report on the effects of a single bout of vigorous physical activity (either of short or long duration, the latter mostly in marathons). However, it is not a single bout but long-term repeated exercise that results in improved physical fitness which is known to be beneficial for the cardiovascular system ${ }^{31,32}$ and is related to reduced mortality. ${ }^{33,34}$ 
To date, the effects of repeated exercise on haemostasis and the underlying mechanisms have yet to be fully discerned, and data on this specific topic is lacking. Therefore, we recently performed a pilot study in which we studied whether repetitive strenuous cycling had an additive, exhaustive or adaptive effect on changes in procoagulant and anticoagulant processes (see Appendix 'Methodology for the Pilot Study'). More data on the effects of training on these variables are becoming available. The current review aims to provide insight into how repeated exercise influences haemostatic parameters (in healthy individuals), by placing it in the context of what is known on the haemostatic effects of training programs and improved training status. We defined "exercise training" as repeated exercise undertaken at a guided or prescribed intensity (either moderate or intensive) and frequency over a specific period of time.

\section{EFFECTS OF EXERCISE TRAINING ON PERIPHERAL BLOOD CELL COUNTS}

Excessive training in athletes has been associated with increased susceptibility to infections, as a result of chronic immunosuppression. ${ }^{35}$ Furthermore, acute exercise is known to induce peripheral leukocytosis. ${ }^{36}$ This leukocyte response is biphasic: after an immediate transient response of lymphocytosis, monocytosis and neutrophilia, a delayed response comprising mainly neutrophilia occurs. Long-term exhaustive endurance exercise such as a marathon or triathlon is predominantly associated with this delayed neutrophilia. ${ }^{37,38}$ The proposed mechanism is an increase in the stress hormone cortisol above $60 \%$ of maximum oxygen uptake $\left(\mathrm{VO}_{2} \mathrm{max}\right) .{ }^{38} \mathrm{~A}$ longitudinal study by Suzuki et al. ${ }^{39}$ addressed the adaptability of these blood cell responses to training. Ten healthy untrained men performed exercise sessions (at an intensity of $70 \%$ of $\mathrm{VO}_{2}$ max) for 1.5 $\mathrm{h}$ each day, for 7 consecutive days. Whereas the first training session caused marked peripheral neutrophilia, in particular band neutrophils, the magnitude of the exerciseinduced changes reduced gradually (although not significantly) by daily repeated exposure to endurance exercise. However, none of the trends were significant except the decline in resting segmented neutrophil counts. ${ }^{39}$

In our cycling study (see Appendix 'Methodology for the Pilot Study') we observed a similar cellular response to repeated exercise (Figure 1), characterized by an increase in granulocytes and decrease in monocytes and lymphocytes on the first day after exercise, followed by recovery to baseline each day before exercise. On the first day, the number of granulocytes was significantly increased after cycling. On the second and third day, this level returned to baseline, but cycling evoked a smaller increase in granulocyte levels compared to the first day. In contrast, monocyte and lymphocyte 
levels decreased significantly on day 1 each day after cycling, but also returned to baseline each day before the cycling. Platelets, red blood cell count, haematocrit and haemoglobin remained largely stable.

\section{EFFECTS OF EXERCISE TRAINING ON HAEMOSTASIS}

Table 1 provides an overview of studies investigating the effect of training on a variety of haemostatic parameters.

\section{Primary haemostasis}

\section{Platelet activity}

Platelet reactivity has a stable association with the occurrence of acute thrombosis and the long-term pathogenesis of thrombosis and cardiovascular diseases. ${ }^{40,41}$ However, the effects of physical training on platelet function have not been fully discerned. It is hypothesized that training in healthy individuals could reduce the risk of cardiovascular disease by suppressing platelet reactivity. Supporting this hypothesis is the observed decrease in exercise-induced platelet hyperreactivity as a result of endurance training in previously sedentary individuals. ${ }^{42-44}$

Two studies investigated the effect of training on platelet activation markers platelet factor 4 (PF4) and beta-thromboglobulin ( $\beta T G)$. In a prospective study, a nine-month endurance training program resulted in slightly increased PF4, but no significant change in $\beta$ TG levels in men and women, suggesting unfavourable training-induced in vivo platelet activation..$^{45}$ In contrast, resting $\beta$ TG levels in physically fit individuals have been reported to be lower compared to sedentary controls ${ }^{42,46}$. Thus, an improved training status appears to have different effects on resting platelet activation than a long-term training program, but further studies in larger populations are warranted to investigate this hypothesis and the underlying mechanism.

In a study by Creighton et al. the haemostatic recovery after exercise in terms of platelet $\beta T G$ and PF4 was monitored with ELISA assays instead of radioimmunoassays. In the first hour post-exercise, resistance-trained individuals displayed significantly reduced platelet activation, in terms of lower $\beta$ TG levels, compared to untrained individuals ${ }^{47}$. Of note, PF4 and $\beta$ TG radioimmunoassays and ELISAs are associated with some methodological difficulties ${ }^{48,49}$. An alternative analysis method for platelet activation is whole blood flow cytometry using antibodies against platelet activation markers, for example GPIb and GPIV. Using this assay, strenuous exercise was found to induce both platelet activation and platelet hyperreactivity in sedentary subjects but not in physically trained subjects. ${ }^{43}$ 
A

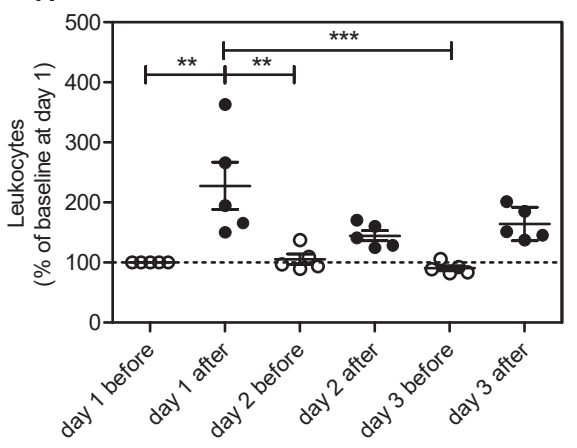

C

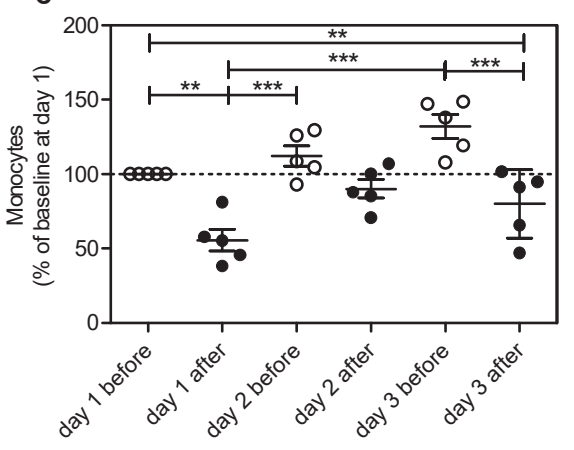

E

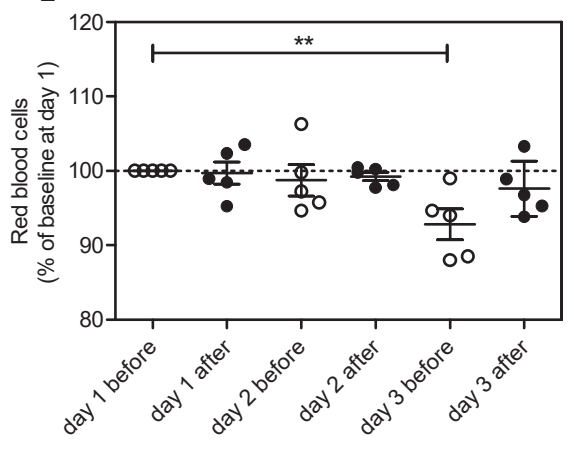

G

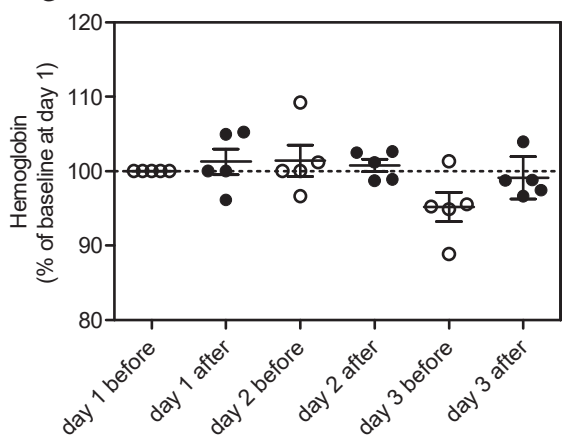

B

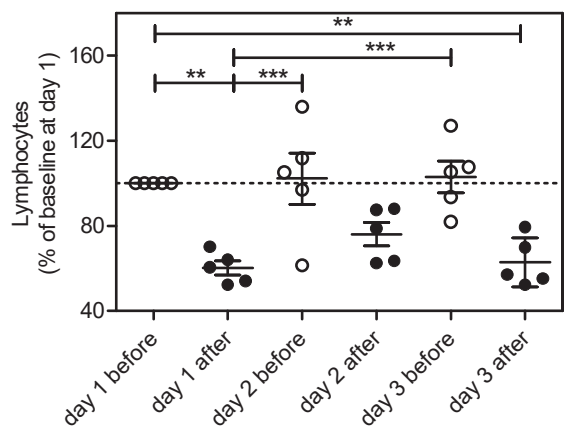

D

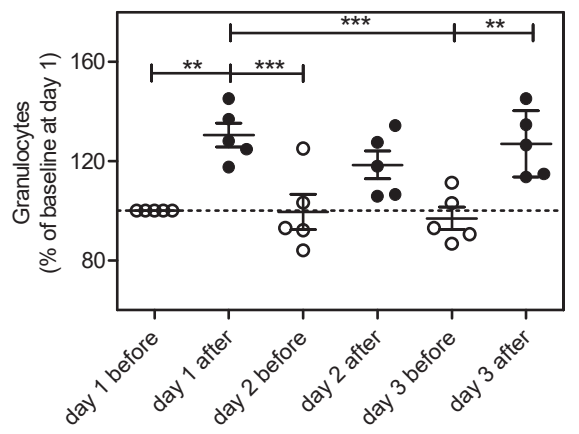

F
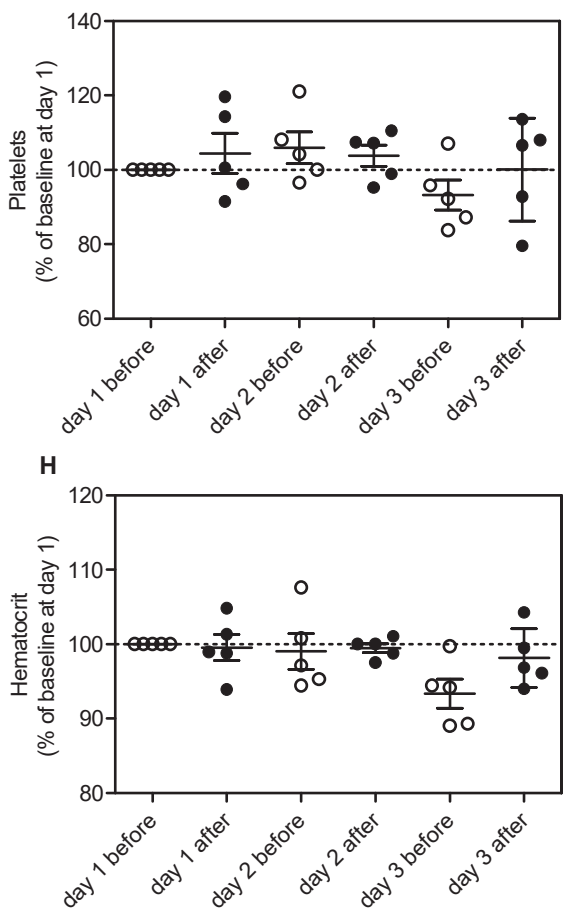
$\leftarrow$ Figure 1. Exercise-induced lymphocytosis, monocytosis and neutrophilia. On the first day, the number of granulocytes was significantly increased after cycling. In contrast, monocyte and lymphocyte levels decreased each day after cycling. On the second and third day, levels returned to baseline. Platelets, red blood cell count, haematocrit and haemoglobin remained largely stable. Data represent the mean $\pm S D(n=5)$.

Table 1. Overview of studies and reported effect of training on haemostatic parameters.

\begin{tabular}{|c|c|c|c|c|}
\hline & Parameter & & Effect & Reference \\
\hline \multirow[t]{14}{*}{ Coagulation } & Clotting times & aPTT & $\downarrow$ & Korsan-Bengtsen ${ }^{51}$ \\
\hline & & aPTT & $\uparrow$ & Hilberg $^{52}$; Kupchak ${ }^{53}$ \\
\hline & & aPTT & $=$ & Ferguson ${ }^{54}$ \\
\hline & & PT & $=$ & Ferguson ${ }^{54} ;$ El-Sayed ${ }^{55} ;$ Korsan-Bengtsen ${ }^{51}$ \\
\hline & & TT & $=$ & Watts $^{56} ;$ El-Sayed ${ }^{55}$ \\
\hline & FVIII & $\mathrm{Ag}$ & $\uparrow$ & Lockard ${ }^{57}$ \\
\hline & & & $=$ & $\begin{array}{l}\text { Boman et al. }{ }^{58} ; \text { Watts }{ }^{56} ; \text { Rankinen }^{59} ; \text { Ponjee }^{45} ; \text { van den } \\
\text { Burg }^{60} \text {; Lippi }{ }^{61} ; \text { Korsan-Bengtsen }\end{array}$ \\
\hline & VWF & $\mathrm{Ag} / \mathrm{Act}$ & $\downarrow$ & Wang ${ }^{44}$ \\
\hline & Thrombin & ETP & $\downarrow$ & Cimenti ${ }^{62}$ \\
\hline & & $\mathrm{F} 1+2$ & $\downarrow$ & Hilberg $^{52}$; Lockard ${ }^{57} ;$ Kupchak $^{53}$ \\
\hline & & TAT & $=$ & Kupchak $^{53}$ \\
\hline & Fibrinogen & $\mathrm{Ag}$ & $\downarrow$ & El-Sayed ${ }^{63} ;$ Stratton $^{64}$ (elderly men) \\
\hline & & & $\uparrow$ & Schuit ${ }^{65}$ (elderly men) \\
\hline & & & $=$ & $\begin{array}{l}\text { Stratton }{ }^{64} \text { (young men); DeSouza }{ }^{66} \text { (elderly women); } \\
\text { Kupchak }^{53}\end{array}$ \\
\hline \multirow[t]{7}{*}{ Platelets } & Aggregation & & $\downarrow$ & Wang $^{44}$ \\
\hline & Activation & P-sel & $\downarrow$ & Santilli ${ }^{67} ;$ Wang $^{44}$ \\
\hline & & VWF bind. & $\downarrow$ & Wang ${ }^{44}$ \\
\hline & & PF4 & $\uparrow$ & Ponjee $^{45}$ \\
\hline & & & $=$ & Creighton $^{68}$ \\
\hline & & $\beta$ TG & $\downarrow$ & Davis $^{42} ;$ Gonzales $^{46} ;$ Creighton $^{68}$ \\
\hline & & GPIb/GPIV & $\downarrow$ & Kestin ${ }^{43}$ \\
\hline \multirow[t]{5}{*}{ Fibrinolysis } & Clot lysis time & & $=$ & Ferguson $^{54}$; Korsan-Bengtsen ${ }^{51}$ \\
\hline & tPA & $\mathrm{Ag} / \mathrm{Act}$ & $\uparrow$ & Szymanski ${ }^{69} ;$ De Paz ${ }^{70} ;$ Kupchak $^{53} ;$ Kulaputana $^{71}$ \\
\hline & PAl-1 & $\mathrm{Ag}$ & $\downarrow$ & Kulaputana ${ }^{71}$ \\
\hline & & & $\uparrow$ & De Geus ${ }^{72}$; Gris ${ }^{73}$; Kupchak ${ }^{53}$; \\
\hline & & & $=$ & Kulaputana ${ }^{71} ;$ Speiser $^{74} ;$ Estelles $^{75}$ \\
\hline
\end{tabular}

aPTT, activated partial thromboplastin time; PT, prothrombin time; TT, thrombin time; Ag, antigen; Act, activity; ETP, endogenous thrombin potential; F1+2, prothrombin fragment 1 and 2; TAT, thrombin-antithrombin complex; P-sel, P-selectin; VWF bind, von Willebrand factor binding; PF4, platelet factor 4; bTG, b thromboglobulin; GPIb/GPIV, glycoprotein Ib/glycoprotein IV; tPA, tissue plasminogen activator; PAI-1, plasminogen activator inhibitor-1. $\uparrow$ increase; $\downarrow$ decrease; = no change, in response to physical training/increased training status. 
In our cycling study platelet activation was also measured in whole blood with a flowcytometric platelet function test (Figure 2)..$^{50}$ Granule release potential was measured as $\mathrm{P}$-selectin expression and the aggregation potential was measured as activation of the $\alpha$ llb $\beta 3$ receptor, both after adding the platelet agonists thrombin receptoractivating peptide (TRAP), collagen-related peptide (CRP) or 2-methylthio-adenosine diphosphate (Mes-ADP). Post-cycling P-selectin expression showed a similar decreasing pattern for protease-activated receptor (PAR-1) stimulation by TRAP and GPVI stimulation by CRP. Interestingly, there was no recovery to baseline the next day before cycling, and P-selectin expression in response to these agonists dropped significantly on the third day. In contrast, $\alpha$ llb $\beta 3$ expression increased slightly after exercise but decreased to baseline for PAR-1, or even lower than baseline for GPVI, on the next day prior to exercise. Mes-ADP-mediated P2Y12 stimulation-induced increased P-selectin expression and $\alpha$ llb $\beta 3$ activation the first day after exercise but remained rather stable on the subsequent days. In summary, repeated exercise predominantly induced an exhaustive effect on platelet granule secretion ( $P$-selectin expression) in response to TRAP and CRP.

\section{Clotting times}

The conventional prothrombin time (PT) and aPTT are measures of overall plasma coagulation via different pathways, and measure the time needed for clot formation; a shortened clotting time indicates a prothrombotic state, whereas increased clotting time indicates hypocoagulability. The PT and aPTT were not different between joggers, marathon runners and individuals with a sedentary lifestyle, both at rest and after exercise. ${ }^{54}$ These findings are in accordance with a later study reporting a similar thrombin time (TT) at rest in athletes and non-athletic individuals. ${ }^{56}$ Moreover, one of the few longitudinal studies investigating the long-term effects of training on coagulation demonstrated no significant change in TT and PT after 3 months of endurance training. ${ }^{55}$ However, although it can be debated whether daily physical activity can be considered training, a large cohort study including 772 men (all aged 54) found that physically very active individuals (assessed by a questionnaire) did have a significantly lower aPTT, indicative of a hypercoagulable state, compared to non-active individuals..$^{51}$ In contrast, one of the few randomized controlled studies found a small but significant prolongation of the aPTT, meaning a more hypo-coagulable state, at rest and post-exercise after 12 weeks of moderate aerobic endurance training..$^{52}$ The latter was confirmed by a more recent study in which trained subjects had a lower clot-forming capacity (prolonged aPTT) following an acute exhaustive resistance exercise test than untrained subjects. ${ }^{53}$ Taken together, data on the effects of training on clotting times are inconclusive. Of note, both PT and aPTT are relatively 'crude' screening tests that may not be sensitive enough to detect hypercoagulability. Therefore, further studies should focus on more sensitive biomarkers of coagulation 

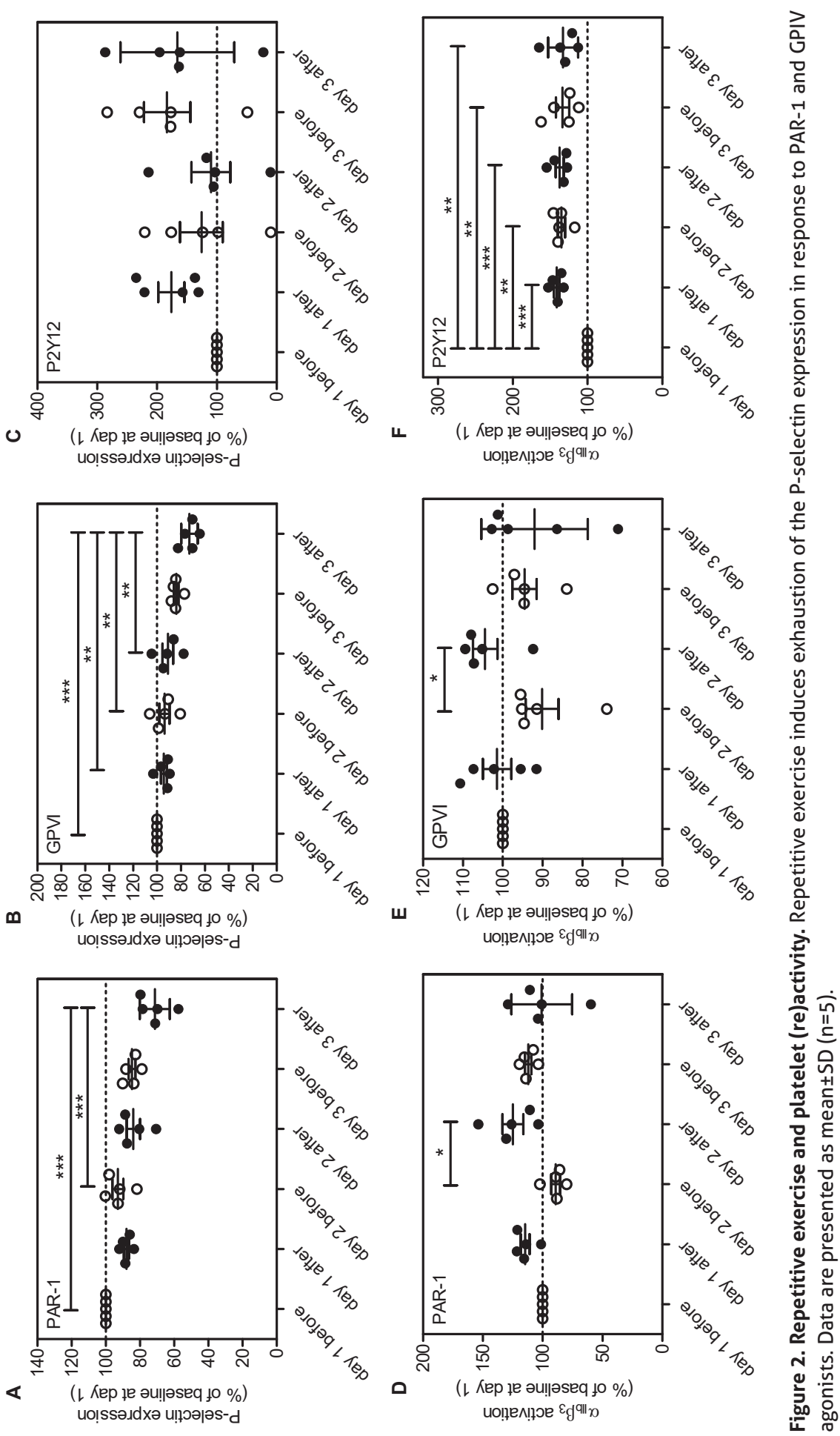


\section{Von Willebrand factor}

von Willebrand factor (VWF) is a large multimeric plasma glycoprotein with essential functions in haemostasis. ${ }^{76}$ Several agonists, including hypoxia, epinephrine, histamine, thrombin, fibrin, and vasopressin, trigger the release of hyperreactive ultra-large VWF multimers from endothelial cell Weibel-Palade bodies. ${ }^{77,78}$ Importantly, this endothelial cell activation also occurs after both short-duration exhaustive exercise ${ }^{30}$ and longduration vigorous exercise $\mathrm{e}^{16-18}$, resulting in an increase in circulating VWF levels.

In our cycling study in five healthy and physically fit individuals, all VWF parameters (VWF antigen VWF:Ag, VWF propeptide (VWFpp) and active VWF) were significantly increased on the first day after cycling (Figures $3 \mathrm{~A}-\mathrm{C}$ ). It is well known that levels of VWF:Ag increase steeply upon acute intense physical exercise ${ }^{79}$. Also, several studies demonstrated exercise-induced elevation of VWF activity, as measured by collagenbinding assays ${ }^{80}$ or ristocetin-cofactor assays. ${ }^{81}$ However, not many studies have determined VWFpp and active VWF (see Appendix 'Methodology for the Pilot Study') levels in response to exercise. VWFpp is critical for the intracellular processing of VWF by endothelial cells. It was previously established that by assaying both VWFpp and VWF:Ag, one can distinguish between acute and chronic endothelial cell damage ${ }^{49}$. In healthy controls, a concomitant rise of VWFpp and VWF:Ag levels, for example, after exercise-induced release from endothelial cell storage is followed by a rapid decline in VWFpp level, whereas VWF:Ag levels return to baseline more slowly. ${ }^{82}$ Active VWF level increases as a result of strenuous exercise due to high intravascular shear stress, which induces VWF to unfold and expose the A1 domain; this conformation is known as active VWF. The observed post-exercise increase in active VWF is in accordance with those reported for the healthy control group in a study on the effects of exercise on VWF in type I and type $2 \mathrm{~B}$ von Willebrand disease patients. ${ }^{81}$ In our cycling study, on the second and third day before cycling all VWF parameters had returned to baseline. Strikingly, on these days all VWF parameters on average increased slightly less in response to cycling compared to the effect on the first day, suggesting that already within 3 days either exhaustion of the endothelial response to physical stress or exhaustion of the VWF supply, similar to tachyphylaxis after repeated DDAVP administration, can be observed. In line with this, the endothelium is known for being modifiable depending on its environment, by mediating pro- and anti-coagulant systems. In fact, a previous study demonstrated that NO-dependent endothelial functions can adapt favourably in as little as one week of endurance training. ${ }^{83}$

Conflicting data are reported on the effect of training status on VWF levels. While VWF antigen (VWF:Ag) levels did not differ between professional cyclists and sedentary individuals ${ }^{61}$, the increase in VWF:Ag upon strenuous exercise appears strongly dependent 
A

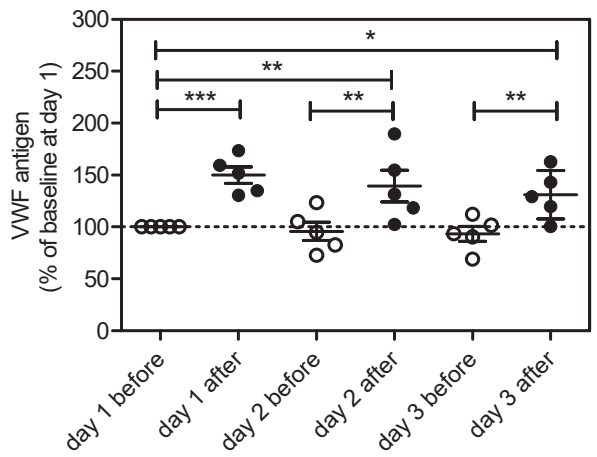

C

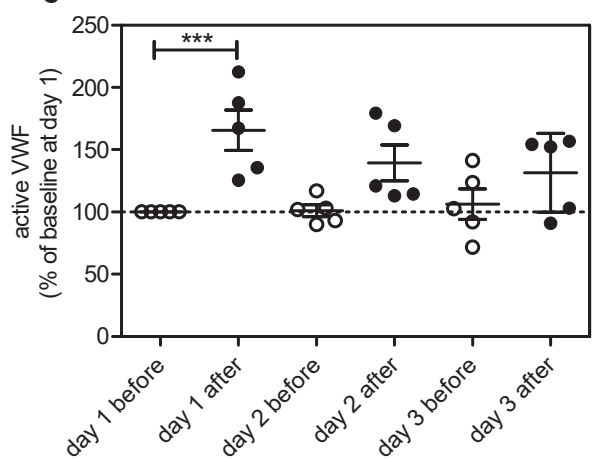

B

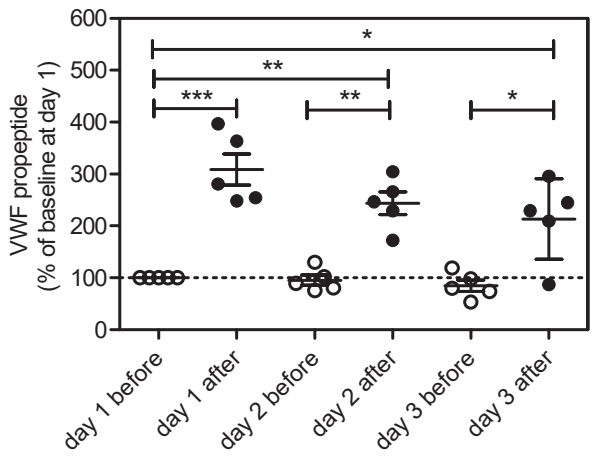

D

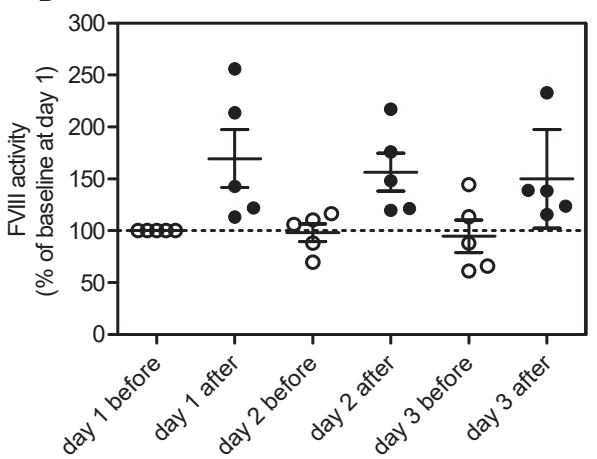

Figure 3. Repetitive exercise induces an endothelial adaptive response. The magnitude of the exercise-induced increase in (A) VWF antigen (VWF:Ag), (B) VWF propeptide (VWF:pp), (C) active VWF and (D) FVIII is less every subsequent day after cycling. (D) FVIII activity was measured as described previously. ${ }^{95,96}$ All measurements were performed in triplicate, and data represent the mean $\pm S D(n=5)$.

on performance- (amongst others peak power output/kg bodyweight and $\mathrm{VO}_{2}$ peak/ $\mathrm{kg}$ ) and physical fitness-related determinants (amongst others $\mathrm{VO}_{2}$ and power output at ventilatory threshold). Interestingly, this increase in VWF:Ag level upon strenuous exercise was highest in individuals who are the least physically fit but also in those who regularly performed very high-intensity exercise. ${ }^{80}$ In a cohort of equally physically fit subjects, an eight-week training program reduced levels of resting and post-exercise VWF:Ag and activity. However, deconditioning completely reverses this training effect. ${ }^{84}$

From a mechanistic point of view, VWF:Ag stored in Weibel-Palade bodies of endothelial cells and platelets may be released more rapidly: (1) as a result of training-induced upregulation of beta2-adrenoreceptors ${ }^{85}$ and, (2) to compensate for shear stress induced by agonists such as thrombin, collagen ${ }^{86}$, epinephrine ${ }^{87}$, and vasopressin ${ }^{88}$. For instance, vasopressin increases blood pressure and vascular resistance during exercise, thereby 
increasing shear stress. Strenuous physical exercise also results in the recruitment of capillaries in muscles and an increase in vascular conductance in these muscles. ${ }^{89}$ As a consequence, the endothelium of trained individuals is exposed to more shear stress and more adrenergic stimulation, which may explain the increased release of VWF upon exercise. ${ }^{90}$ Of note, shear stress is also known to unfold VWF multimers, exposing the A1 domain. ${ }^{91}$ This active conformation of VWF can more readily bind platelets and is thus more thrombogenic. ${ }^{92,93}$

In conclusion, exposure to exercise in trained individuals may facilitate a more rapid return of haemostatic parameters such as VWF to resting conditions. Hence, if this hypothesis would be true, the release of VWF:Ag may be briefer in trained subjects. ${ }^{94}$

\section{Secondary haemostasis}

\section{FVIII}

Resting levels of FVIII activity and FVIII antigen do not change with training in physically $\mathrm{fit}^{56}$, as well as sedentary individuals in the majority of studies. ${ }^{45,55,58-61}$ In a more recent study, FVIII:Ag levels did increase significantly with training ${ }^{57}$, consistent with the observed increase in FVIII activity after acute exercise. ${ }^{25,30,97,98}$ However, this inconsistency may be explained by population differences, as the latter study included elderly men and women (50-75 years) whereas all other studies mentioned previously were performed with young individuals. In our cycling study, the submaximal intensity cycling induced an increase in FVIII activity on each of 3 subsequent days (Figure 3D). Interestingly, the difference between pre- and post-exercise FVIII levels became smaller every day, indicating either adaptation of the FVIII response to exercise or exhaustion of the FVIII supplies. FVIII circulates in complex with VWF, therefore high FVIII levels correlate with an increased level of VWF. Of note, the aforementioned shortening of the aPTT and the elevation in FVIII activity induced by acute strenuous exercise are both indicators for contact factor-mediated clotting activity. Hence, this pathway may have an important role in post-exercise hypercoagulability ${ }^{99}$, but does not appear subject to an adaptive response induced by training.

\section{Thrombin}

In contrast to global clotting assays such as the PT and aPTT, measurement of thrombin generation (TG) allows assessment of the full process of coagulation over time, providing greater sensitivity. ${ }^{100}$ Thrombin formation is lower in trained versus sedentary subjects as shown by a lower resting endogenous thrombin potential (ETP) ${ }^{69}$ and lower level of prothrombin fragments $\mathrm{F} 1+2$, a surrogate thrombin marker. ${ }^{57}$ In addition, in endurance-trained subjects, the increase of thrombin formation response to exercise is less pronounced compared to controls. ${ }^{64}$ 
Regarding the short-term effect of repetitive exercise on TG, we observed in our cycling study that peak TG was significantly increased on the first day following exercise (Figure 4A). However, peak TG returned to baseline before cycling on the second and third day. This is in accordance with findings from a recent study in which an acute bout of highintensity exercise increased TG immediately after exercise, but returned to baseline the following day. ${ }^{20}$ Remarkably, almost no increase in ETP was observed after cycling on the second and third day. The ETP before exercise actually decreased over the three days of cycling (Figure 4B). Together, these results show an initial surge, followed by exhaustion of the thrombin generating capacity of the haemostatic system. This pattern possibly reflects the observed exercise-induced increase in FVIII with a tapered effect over the 3 days. Our study only lasted 3 days, but these results warrant further investigations to determine whether the drop in TG continues further and how much time after exhaustion is required for TG to return to baseline.

Overall, the apparent favourable effect of training on clotting potential may explain the lower incidence of thrombosis in physically fit individuals, despite the transiently hypercoagulable state during and directly after strenuous exercise.

\section{Fibrinogen}

Fibrinogen plays a pivotal role in normal haemostasis, representing the substrate for conversion to fibrin and supporting TC and platelet aggregation. ${ }^{101}$ Studies on the effects of exercise on fibrinogen levels have produced conflicting data, reporting significant increases, decreases, or no effects. ${ }^{30,69,97,102-104}$ These inconsistencies in the literature can likely be attributed to differences in experimental design, including duration, mode and intensity of exercise, health and training status of the study population and analytical methods. ${ }^{30,97,102}$ Whereas most studies determined the effects of acute exercise, the influence of physical training on plasma fibrinogen levels is less well studied. Cross-sectional studies suggest a favourable effect of regular physical activity, decreasing plasma fibrinogen levels. ${ }^{63,105}$ However, longitudinal data is sparse and conflicting. Physical training at moderate intensity reduced plasma fibrinogen levels in elderly men but not in young men.${ }^{64}$ In contrast, following intensive training plasma fibrinogen levels were increased in elderly men, paralleled by a surge in C-reactive protein suggesting a chronic increase in acute-phase reactant proteins. ${ }^{65}$ In elderly women, on the other hand, no significant effects of training on fibrinogen levels were found. ${ }^{66}$ Similarly, in our cycling study in men, no significant changes in fibrinogen plasma levels in response to repeated bouts of submaximal exercise were observed (Figure 4C). Altogether, there is a lack of conclusive evidence on the exact effects of physical training on plasma fibrinogen levels. 


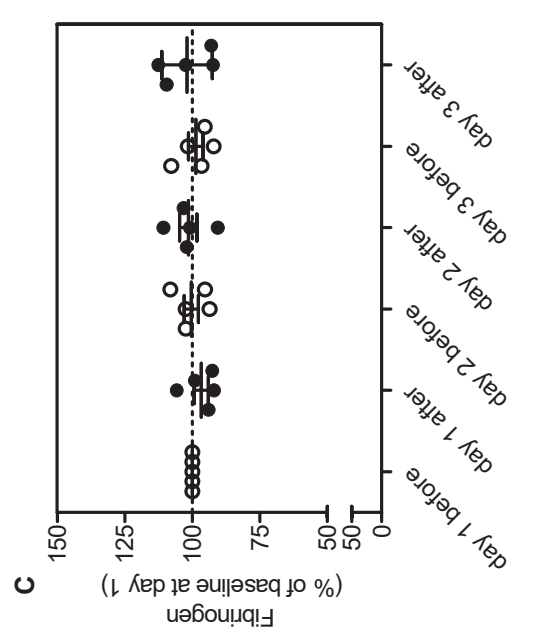

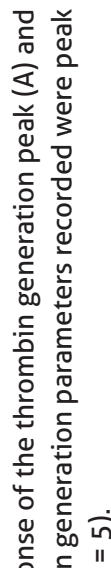

ᄃิ

ญे है О

. 녿

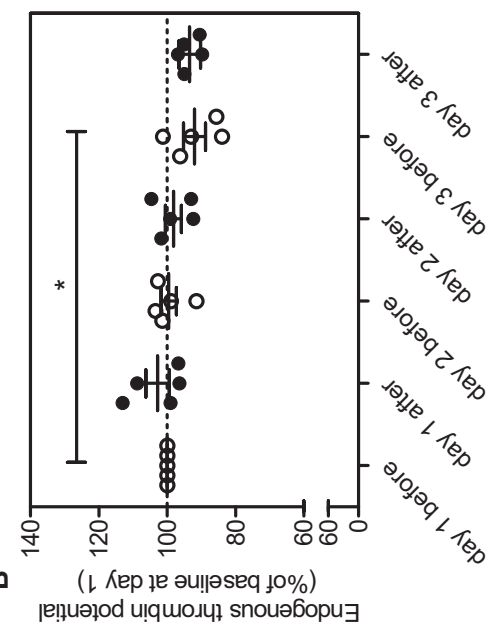

ㅇํํ

ֻัฒ

范

ปั ऽ ญ

ํํㄷำ

는 운

気 㫕

ชิ

.

芉

웜 음

范范

웅

垴

品 㔛

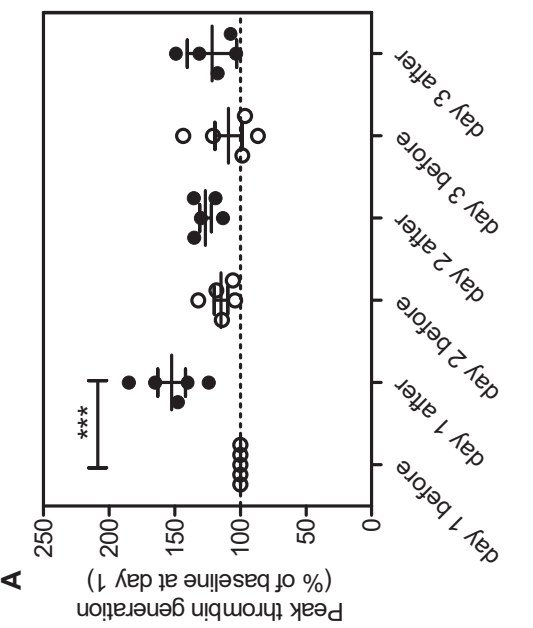

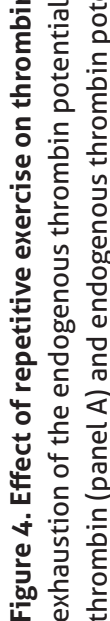




\section{Fibrinolysis}

Despite the hypercoagulable state that occurs during and directly after strenuous exercise, regular physical activity has been associated with a reduction in cardiovascular risk. ${ }^{106-108}$ The underlying mechanisms of this conditioning effect remain speculative, but are often ascribed to favourable effects on fibrinolysis. ${ }^{55,109-111}$ However, as with the effects on coagulation, studies on the effects of training on fibrinolysis parameters have produced inconsistent results. Global assays of fibrinolysis, for example the clot lysis time, found no difference in fibrinolytic capacity between marathon runners and less active individuals. ${ }^{51,54}$ In our small-scale cycling study, clot lysis time decreased only very slightly on the first two days after exercise and decreased significantly on the third day (Figure 5). When assaying specific fibrinolytic factors, the effects of exercise could be observed. For instance, inactive individuals have higher tPA activity and tPA antigen levels compared to active individuals. ${ }^{69,70}$ Data on PAI-1 levels following training are inconclusive. A favourable reduction in PAl-1 activity was observed after 8 months of training (albeit not significant due to large group variances and seasonal variations) ${ }^{72}$ and after 3 months of training. ${ }^{73}$ Detraining for 3 months in the latter study reversed the reduction in PAI-1 activity. ${ }^{73}$ On the other hand, PAI-1 values were increased in athletes compared with age-matched sedentary individuals and elderly sportsmen ${ }^{74}$ and an exercise rehabilitation program did not significantly reduce PAI-1 levels in healthy controls. $^{75}$

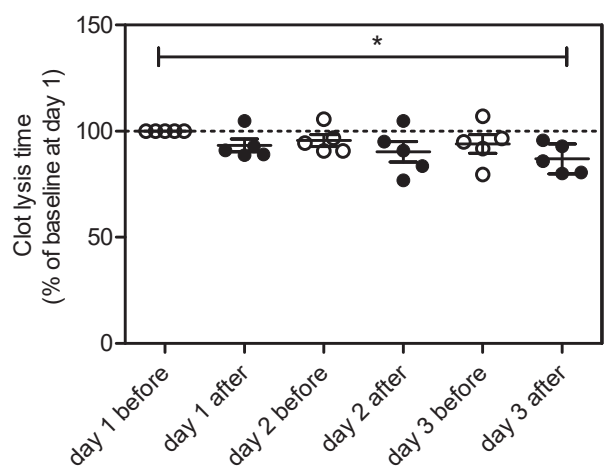

Figure 5. Repetitive exercise causes only a minor decrease of clot lysis time (CLT). The clot lysis time (CLT; $50 \%$ fibrinolysis) is defined as the time between maximal and half-maximal turbidity. Data are presented as mean $\pm S D(n=$ 5).

Several mechanisms may be responsible for the training-induced adaptation of fibrinolysis. One explanation is the enhanced sensitivity of the endothelium to release tPA, possibly paralleled by reduced clearance of tPA by the liver. In addition, the formation of tPA/PAl-1 complexes is reduced ${ }^{70,112}$, which may be related to exercise-induced changes in lipid profile that lower resting PAl-1 activity. ${ }^{97,113,114}$ Catecholamines may also play a role in training-related changes in fibrinolysis through their effects on the endothelium. However, data on the magnitude of the effect of plasma epinephrine and 
norepinephrine are equivocal, and hence the enhanced sensitivity of the endothelium to release tPA may be related to other, non-adrenergic mechanisms.

In conclusion, although the available evidence is derived from highly heterogeneous study protocols, training may have a positive influence on fibrinolysis in terms of an increase in TPA levels and a reduction in PAI-1 levels, although this must be verified in future studies before a definitive conclusion can be drawn.

\section{MODIFIERS OF THE EFFECTS OF TRAINING ON HAEMOSTASIS}

\section{Lipid profile and nitric oxide}

Changes in lipid profile and plasma nitric oxide (NO) levels (as measured by NO metabolites) may modulate platelet reactivity in trained subjects. ${ }^{44}$ Intensive repeated exercise resulted in significantly decreased thromboxane (TX)-dependent platelet activation (urinary excretion of 11-dehydroTXB2, enzymatic metabolite of TXA2), TX-independent platelet activation (plasma P-selectin) and platelet-derived inflammatory proteins (plasma CD4OL) in otherwise sedentary subjects. Concomitantly, a significant increase in HDL cholesterol concentration, linearly and inversely related to changes in the Pselectin and CD40L platelet activation markers, was observed. ${ }^{67}$ Indeed, HDL is known to engage in a large number of beneficial activities at the endothelial level, amongst others stimulating NO synthase and inhibiting platelet activation and aggregation ${ }^{115}$, which may explain mechanistically how this type of high intensity regular physical training may reduce the risk of cardiovascular events. ${ }^{116}$

\section{Catecholamines}

Regular exercise and training decreases surges in catecholamines, both at rest and following exercise. ${ }^{26}$ Only one week of vigorous exercise training was sufficient to induce significant reductions in the norepinephrine response to the same workload. ${ }^{117}$ However, catecholamines have distinct effects on different key players in blood coagulation. For example, training reduces the density and affinity of platelet surface adrenergic receptors. Together with a decreased release of catecholamines in response to exercise this leads to reduced overall platelet activity and aggregation under high shear flow. ${ }^{118,119}$ In contrast, training-induced up-regulation of endothelial 2-adrenoreceptors causes accelerated release of FVIII and VWF ${ }^{85}$, which may result in a more pro-coagulant phenotype after training. 


\section{Dehydration}

During exercise, sweatingand intravascular fluid shifts into the interstitial space result in decreased plasma volume without a proportional loss of plasma proteins. ${ }^{120}$ This haemoconcentration may result in increased post-exercise (coagulation) protein levels (falsely suggesting increased activation of the coagulation system) and changes in RBC count, haemoglobin and haematocrit. ${ }^{120}$ However, whereas in most studies on the effects of short term exercise plasma volume was decreased after exercise, coagulation factor levels did not change. ${ }^{121-123}$ In one report an increased haematocrit was accompanied by increased levels of factors IX, XI, XII, but no changes in other coagulation factors were observed, suggesting actual activation of the contact pathway of coagulation. ${ }^{25}$ In our cycling study, no changes in haematocrit and haemoglobin and a minor decrease in RBC count were observed. Hence, haemoconcentration is not expected to distort our findings of increased FVIII levels, TG and VWF following exercise.

\section{Training status and mode}

One factor that modifies the effect of physical activity on fibrinolysis is the training status of the individual. ${ }^{54}$ The activating effects of exercise on haemostasis appear more pronounced in sedentary compared to physically fit individuals. ${ }^{79}$ For instance, strenuous exercise caused significant platelet hyperactivation in sedentary men, but not in regularly-exercising subjects. ${ }^{43,117}$ Likewise, post-training tPA release was increased and tPA/PAl-1 complex decreased in physically trained subjects compared with untrained individuals. $^{69,70}$ Training status influences an individual's maximal aerobic capacity, and studies have found that the level of acceleration of fibrinolytic activity is directly related to the workload, suggesting that higher aerobic fitness may lead to a larger increase in fibrinolysis with maximal exercise. ${ }^{54,97}$ Exercise mode may also influence the haemostatic response to training. The above-mentioned studies show that endurance training seems to condition platelets to become less activated and aggregate less in response to strenuous exercise. ${ }^{43,44}$ In contrast, resistance training is believed to enhance haemostatic functions by increasing vessel diameter and promoting antithrombotic endothelial activity. ${ }^{124-127}$

\section{Age}

The favourable effects of training on blood fibrinolysis may be at least partly dependent on age. In the elderly, there is a higher fibrinolytic capacity, characterized by increased tPA and decreased PAl-1 activity ${ }^{64}$ and -antigen ${ }^{65}$ after training, compared to young individuals. However, another study reported that physical training can also positively influence blood fibrinolysis in younger individuals. ${ }^{60}$ In addition, plasma fibrinogen levels reduced in response to training in older individuals, but not in young subjects. ${ }^{64,79}$ Other studies have found improvements in haemostatic markers, including PT, FVIII, pro- 
thrombin F1+2 and VWF in subjects between 50 and 75 years old but not in a younger population. ${ }^{128,129}$

\section{Gender}

Most studies examining the haemostatic effects of training included only male subjects. The response of peripheral blood cell counts and D-dimer levels to a single bout of exercise appear the same in both genders, whereas the post-exercise clotting times decreased more in males than females. ${ }^{130}$ The sparse data available for repetitive exercise suggests that gender may modulate the fibrinolytic response to training, as endurance training-induced improvements in endogenous fibrinolysis markers, i.e. a decrease in PAI-1 and increase in tPA levels, are somewhat greater in men compared to women. ${ }^{71}$

\section{CONCLUSIONS}

Although exercise is established to be essential for a healthy lifestyle, there are many reports of exercise-induced thrombotic events. This so-called exercise paradox ${ }^{11,12}$ reflects the complex interplay between haemostasis and physical stress. Whereas the risk of a cardiovascular event after vigorous exercise is increased in untrained individuals, training might induce adaptations of the haemostatic system, explaining its favourable effects on cardiovascular health and mortality.

Primary and secondary haemostasis, as well as fibrinolysis, are all affected by training. However, the exact effects of exercise and training on the haemostatic profile and the underlying mechanisms remain to be elucidated. Inconsistencies in reported data, as apparent from Table 1, can be attributed to variation in subject-related factors such as age, gender, diet and training status, as well as activity-related factors such as intensity, duration and mode of exercise. While the latter factors can be standardized by investigators, study populations are generally heterogeneous, precluding definite conclusions on the size and direction of the effects of repeated exercise on pro- and anticoagulant processes.

Furthermore, almost no data on the short-term effects of repeated exercise are reported in the literature. For this reason, we performed a pilot study in which five individuals cycled at submaximal intensity for three subsequent days. The results of this pilot study suggest that repeated submaximal exercise leads to exhaustion of several components of the haemostatic system, predominantly of the endothelium (apparent from changes in VWF and FVIII) and platelet granule secretion (as measured by P-selectin expression). 
However, we acknowledge several limitations of our pilot study that could have influenced the measured haemostatic parameters. First of all, the limited reproducibility of our 'exercise protocol', i.e. cycling a specific route in the hilly landscape in the south of the Netherlands, and the inability to monitor and control exercise intensity are important weaknesses of this study. Therefore, in further studies it is recommended to perform a standardized submaximal exercise workload on a cycling ergometer. In addition, participants were considered physically fit based on their self-reported frequency and intensity of exercise, but this was not objectively determined (e.g. in terms of aerobic capacity $V_{02 \text { max }}$ ) prior to the pilot study. Thus, observed differences in haemostatic response to bouts of strenuous exercise may be partially accounted for by differences in baseline fitness. Finally, participants consumed water, bananas, energy drinks, energy bars and energy gels ad libitum during cycling, but the individual fluid and food intake were not recorded.

Several questions related to the effects of repetitive exercise and haemostasis remain unanswered and warrant further investigation. For instance, there is a large gap in knowledge on the effects of training on a crucial factor in fibrinolysis, namely plasminogen levels and subsequent plasmin generation. Moreover, it is highly relevant to obtain better estimates of the impact of training status on the incidence of exercise-induced thrombotic events, and how this compares to the effects of therapeutic intervention. Ideally, these studies should include a longitudinal design with a follow up of several years. In addition, new research should focus on biomarkers of training-induced endothelial activation, in particular (active) VWF. Finally, more studies on short-term repetitive exercise on haemostasis are required, as the effects on haemostasis appear different than those induced by long-term repetitive exercise in the form of training. Altogether, insights into the effects of training on haemostatic disturbances by exercise will form the basis to make the double-edged sword of exercise cut in the favourable direction, protecting against thromboembolic morbidity and mortality.

\section{ACKNOWLEDGEMENTS}

We would like to thank the volunteers for their participation in this study and Suzanne Zwaveling, Martijn Moorlag and Viola Strijbis for their help with blood collection and experiments. 


\section{REFERENCES}

1. Rutten B, Maseri A, Cianflone D, Laricchia A, Cristell NA, Durante A, et al. Plasma levels of active Von Willebrand factor are increased in patients with first ST-segment elevation myocardial infarction: a multicenter and multiethnic study. Eur Heart J Acute Cardiovasc Care. 2015;4(1):64-74.

2. Shiroma EJ, Lee IM. Physical activity and cardiovascular health: lessons learned from epidemiological studies across age, gender, and race/ethnicity. Circulation. 2010;122(7):743-52.

3. Lavie CJ, Thomas RJ, Squires RW, Allison TG, Milani RV. Exercise training and cardiac rehabilitation in primary and secondary prevention of coronary heart disease. Mayo Clin Proc. 2009;84(4):373-83.

4. Pollock ML, Franklin BA, Balady GJ, Chaitman BL, Fleg JL, Fletcher B, et al. AHA Science Advisory. Resistance exercise in individuals with and without cardiovascular disease: benefits, rationale, safety, and prescription: An advisory from the Committee on Exercise, Rehabilitation, and Prevention, Council on Clinical Cardiology, American Heart Association; Position paper endorsed by the American College of Sports Medicine. Circulation. 2000;101(7):82833.

5. Swift DL, Lavie CJ, Johannsen NM, Arena R, Earnest CP, O'Keefe JH, et al. Physical activity, cardiorespiratory fitness, and exercise training in primary and secondary coronary prevention. Circ J. 2013;77(2):281-92.

6. Kopperstad O, Skogen JC, Sivertsen B, Tell GS, Saether SM. Physical activity is independently associated with reduced mortality: 15-years follow-up of the Hordaland Health Study (HUSK). PLoS One. 2017;12(3):e0172932.

7. Lippi G, Schena F, Salvagno GL, Montagnana M, Ballestrieri F, Guidi GC. Comparison of the lipid profile and lipoprotein(a) between sedentary and highly trained subjects. Clin Chem Lab Med. 2006;44(3):322-6.

8. Swift DL, Johannsen NM, Lavie CJ, Earnest CP, Church TS. The role of exercise and physical activity in weight loss and maintenance. Prog Cardiovasc Dis. 2014;56(4):441-7.

9. Little JP, Francois ME. High-intensity interval training for improving postprandial hyperglycemia. Res O. Exerc Sport. 2014;85(4):451-6.

10. Thijssen DH, Tinken TM, Hopkins N, Dawson EA, Cable NT, Green DJ. The impact of exercise training on the diameter dilator response to forearm ischaemia in healthy men. Acta Physiol (Oxf). 2011;201(4):427-34.

11. Maron BJ. The paradox of exercise. N Engl J Med. 2000;343(19):1409-11.

12. Parto P, O'Keefe JH, Lavie CJ. The Exercise Rehabilitation Paradox: Less May Be More? Ochsner J. 2016;16(3):297-303.

13. Albert CM, Mittleman MA, Chae CU, Lee IM, Hennekens CH, Manson JE. Triggering of sudden death from cardiac causes by vigorous exertion. N Engl J Med. 2000;343(19):1355-61.

14. Lippi G, Maffulli N. Biological influence of physical exercise on hemostasis. Semin Thromb Hemost. 2009;35(3):269-76.

15. Schmied C, Borjesson M. Sudden cardiac death in athletes. J Intern Med. 2014;275(2):93103.

16. Thompson PD, Franklin BA, Balady GJ, Blair SN, Corrado D, Estes NA, 3rd, et al. Exercise and acute cardiovascular events placing the risks into perspective: a scientific statement from the American Heart Association Council on Nutrition, Physical Activity, and Metabolism and the Council on Clinical Cardiology. Circulation. 2007;115(17):2358-68. 
17. Borissoff Jl, Spronk HM, ten Cate H. The hemostatic system as a modulator of atherosclerosis. N Engl J Med. 2011;364(18):1746-60.

18. Hilberg T, Prasa D, Stürzebecher J, Gläser D, Gabriel HHW. Thrombin Potential and Thrombin Generation After Exhaustive Exercise. Int J Sports Med. 2002;23(07):500-4.

19. Gunga HC, Kirsch K, Beneke R, Boning D, Hopfenmuller W, Leithauser R, et al. Markers of coagulation, fibrinolysis and angiogenesis after strenuous short-term exercise (Wingatetest) in male subjects of varying fitness levels. Int J Sports Med. 2002;23(7):495-9.

20. Sedgwick MJ, Thompson M, Garnham J, Thackray AE, Barrett LA, Powis M, et al. Acute highintensity interval rowing increases thrombin generation in healthy men. Eur ] Appl Physiol. 2016;116(6):1139-48.

21. Hilberg T, Prasa D, Sturzebecher J, Glaser D, Schneider K, Gabriel HH. Blood coagulation and fibrinolysis after extreme short-term exercise. Thromb Res. 2003;109(5-6):271-7.

22. Weiss C, Bierhaus A, Kinscherf R, Hack V, Luther T, Nawroth PP, et al. Tissue factor-dependent pathway is not involved in exercise-induced formation of thrombin and fibrin. J Appl Physiol (1985). 2002;92(1):211-8.

23. Posthuma JJ, van der Meijden PE, Ten Cate H, Spronk HM. Short- and Long-term exercise induced alterations in haemostasis: a review of the literature. Blood Rev. 2015;29(3):171-8.

24. Zadow EK, Kitic CM, Wu SSK, Fell JW, Adams MJ. Time of day and short-duration highintensity exercise influences on coagulation and fibrinolysis. European Journal of Sport Science. 2018;18(3):367-75.

25. Menzel K, Hilberg T. Blood coagulation and fibrinolysis in healthy, untrained subjects: effects of different exercise intensities controlled by individual anaerobic threshold. Eur ] Appl Physiol. 2011;111(2):253-60.

26. Wang JS. Exercise prescription and thrombogenesis. J Biomed Sci. 2006;13(6):753-61.

27. Siscovick DS, Weiss NS, Fletcher RH, Lasky T. The incidence of primary cardiac arrest during vigorous exercise. N Engl J Med. 1984;311(14):874-7.

28. Wang JS, Jen CJ, Kung HC, Lin LJ, Hsiue TR, Chen HI. Different effects of strenuous exercise and moderate exercise on platelet function in men. Circulation. 1994;90(6):2877-85.

29. Eijsvogels TM, George KP, Thompson PD. Cardiovascular benefits and risks across the physical activity continuum. Curr Opin Cardiol. 2016;31(5):566-71.

30. El-Sayed MS, El-Sayed Ali Z, Ahmadizad S. Exercise and training effects on blood haemostasis in health and disease: an update. Sports Med. 2004;34(3):181-200.

31. Stewart KJ. Exercise training and the cardiovascular consequences of type 2 diabetes and hypertension: plausible mechanisms for improving cardiovascular health. JAMA. 2002;288(13):1622-31.

32. Yoshikawa D, Ishii H, Kurebayashi N, Sato B, Hayakawa S, Ando H, et al. Association of cardiorespiratory fitness with characteristics of coronary plaque: assessment using integrated backscatter intravascular ultrasound and optical coherence tomography. Int J Cardiol. 2013;162(2):123-8.

33. Blair SN, Kohl HW, 3rd, Barlow CE, Paffenbarger RS, Jr., Gibbons LW, Macera CA. Changes in physical fitness and all-cause mortality. A prospective study of healthy and unhealthy men. JAMA. 1995;273(14):1093-8.

34. Kokkinos P, Myers J, Faselis C, Panagiotakos DB, Doumas M, Pittaras A, et al. Exercise capacity and mortality in older men: a 20-year follow-up study. Circulation. 2010;122(8):790-7. 
35. Gunzer W, Konrad M, Pail E. Exercise-induced immunodepression in endurance athletes and nutritional intervention with carbohydrate, protein and fat-what is possible, what is not? Nutrients. 2012;4(9):1187-212.

36. Rowbottom DG, Green KJ. Acute exercise effects on the immune system. Med Sci Sports Exerc. 2000;32(7 Suppl):S396-405.

37. Gleeson M. Immune function in sport and exercise. J Appl Physiol (1985). 2007;103(2):6939.

38. Catanho da Silva FO, Macedo DV. Physical exercise, inflammatory process and adaptive condition: an overview. . Braz J Kinanthropometry and Human Performance. 2011;13(4):320-8.

39. Suzuki K, Naganuma S, Totsuka M, Suzuki KJ, Mochizuki M, Shiraishi M, et al. Effects of exhaustive endurance exercise and its one-week daily repetition on neutrophil count and functional status in untrained men. Int J Sports Med. 1996;17(3):205-12.

40. Olgun N, Uysal KM, Irken G, Unal N, Undar B, Akkoc N, et al. Platelet activation in congenital heart diseases. Acta Paediatr Jpn. 1997;39(5):566-9.

41. Wu KK. Hemostatic tests in the prediction of atherothrombotic disease. Int ] Clin Lab Res. 1997;27(3):145-52.

42. Davis RB, Boyd DG, McKinney ME, Jones CC. Effects of exercise and exercise conditioning on blood platelet function. Med Sci Sports Exerc. 1990;22(1):49-53.

43. Kestin AS, Ellis PA, Barnard MR, Errichetti A, Rosner BA, Michelson AD. Effect of strenuous exercise on platelet activation state and reactivity. Circulation. 1993;88(4 Pt 1):1502-11.

44. Wang JS, Jen $\mathrm{CJ}$, Chen HI. Effects of exercise training and deconditioning on platelet function in men. Arterioscler Thromb Vasc Biol. 1995;15(10):1668-74.

45. Ponjee GA, Janssen GM, van Wersch JW. Prolonged endurance exercise and blood coagulation: a 9 month prospective study. Blood Coagul Fibrinolysis. 1993;4(1):21-5.

46. Gonzales F, Manas M, Seiquer I, Quiles J, Mataix FJ, Huertas JR, et al. Blood platelet function in healthy individuals of different ages. Effects of exercise and exercise conditioning. J Sports Med Phys Fitness. 1996;36(2):112-6.

47. Creighton BC, Kupchak BR, Aristizabal JC, Flanagan SD, Dunn-Lewis C, Volk BM, et al. Influence of training on markers of platelet activation in response to a bout of heavy resistance exercise. European Journal of Applied Physiology. 2013;113(9):2203-9.

48. Kestin AS, Ellis PA, Barnard MR, Errichetti A, Rosner BA, Michelson AD. Effect of strenuous exercise on platelet activation state and reactivity. Circulation. 1993;88(4):1502-11.

49. Murakami T, Komiyama Y, Masuda M, Kido H, Nomura S, Fukuhara S, et al. Flow cytometric analysis of platelet activation markers CD62P and CD63 in patients with coronary artery disease. European Journal of Clinical Investigation. 1996;26(11):996-1003.

50. Roest M, van Holten TC, Fleurke GJ, Remijn JA. Platelet Activation Test in Unprocessed Blood (Pac-t-UB) to Monitor Platelet Concentrates and Whole Blood of Thrombocytopenic Patients. Transfus Med Hemother. 2013;40(2):117-25.

51. Korsan-Bengtsen $\mathrm{K}$, Wilhelmsen $\mathrm{L}$, Tibblin $\mathrm{G}$. Blood coagulation and fibrinolysis in relation to degree of physical activity during work and leisure time. A study based on a random sample of 54-year-old men. Acta Med Scand. 1973;193(1-2):73-7.

52. Hilberg T, Menzel K, Wehmeier UF. Endurance training modifies exercise-induced activation of blood coagulation: RCT. Eur J Appl Physiol. 2013;113(6):1423-30.

53. Kupchak BR, Creighton BC, Aristizabal JC, Dunn-Lewis C, Volk BM, Ballard KD, et al. Beneficial effects of habitual resistance exercise training on coagulation and fibrinolytic responses. Thromb Res. 2013;131(6):e227-34. 
54. Ferguson EW, Bernier LL, Banta GR, Yu-Yahiro J, Schoomaker EB. Effects of exercise and conditioning on clotting and fibrinolytic activity in men. J Appl Physiol (1985). 1987;62(4):141621.

55. El-Sayed MS, Lin X, Rattu AJ. Blood coagulation and fibrinolysis at rest and in response to maximal exercise before and after a physical conditioning programme. Blood Coagul Fibrinolysis. 1995;6(8):747-52.

56. Watts EJ. Haemostatic changes in long-distance runners and their relevance to the prevention of ischaemic heart disease. Blood Coagul Fibrinolysis. 1991;2(2):221-5.

57. Lockard MM, Gopinathannair R, Paton CM, Phares DA, Hagberg JM. Exercise training-induced changes in coagulation factors in older adults. Med Sci Sports Exerc. 2007;39(4):587-92.

58. Boman K, Hellsten G, Bruce A, Hallmans G, Nilsson TK. Endurance physical activity, diet and fibrinolysis. Atherosclerosis. 1994;106(1):65-74.

59. Rankinen T, Rauramaa R, Vaisanen S, Halonen P, Penttila IM. Blood coagulation and fibrinolytic factors are unchanged by aerobic exercise or fat modified diet. Fibrinolysis. 1994;8:48-53.

60. van den Burg PJ, Hospers JE, van Vliet M, Mosterd WL, Bouma BN, Huisveld IA. Effect of endurance training and seasonal fluctuation on coagulation and fibrinolysis in young sedentary men. J Appl Physiol (1985). 1997;82(2):613-20.

61. Lippi G, Salvagno GL, Montagana M, Guidi GC. Chronic influence of vigorous aerobic training on hemostasis. Blood Coagul Fibrinolysis. 2005;16(7):533-4.

62. Cimenti C, Schlagenhauf A, Leschnik B, Schretter M, Tschakert G, Groschl W, et al. Low endogenous thrombin potential in trained subjects. Thromb Res. 2013;131(6):e281-5.

63. el-Sayed MS. Effects of high and low intensity aerobic conditioning programs on blood fibrinolysis and lipid profile. Blood Coagul Fibrinolysis. 1996;7(4):484-90.

64. Stratton JR, Chandler WL, Schwartz RS, Cerqueira MD, Levy WC, Kahn SE, et al. Effects of physical conditioning on fibrinolytic variables and fibrinogen in young and old healthy adults. Circulation. 1991;83(5):1692-7.

65. Schuit AJ, Schouten EG, Kluft C, de Maat M, Menheere PP, Kok FJ. Effect of strenuous exercise on fibrinogen and fibrinolysis in healthy elderly men and women. Thromb Haemost. 1997;78(2):845-51.

66. DeSouza CA, Jones PP, Seals DR. Physical activity status and adverse age-related differences in coagulation and fibrinolytic factors in women. Arterioscler Thromb Vasc Biol. 1998;18(3):362-8.

67. Santilli F, Vazzana N, lodice P, Lattanzio S, Liani R, Bellomo RG, et al. Effects of high-amounthigh-intensity exercise on in vivo platelet activation: modulation by lipid peroxidation and AGE/RAGE axis. Thromb Haemost. 2013;110(6):1232-40.

68. Creighton BC, Kupchak BR, Aristizabal JC, Flanagan SD, Dunn-Lewis C, Volk BM, et al. Influence of training on markers of platelet activation in response to a bout of heavy resistance exercise. Eur J Appl Physiol. 2013;113(9):2203-9.

69. Szymanski LM, Pate RR, Durstine JL. Effects of maximal exercise and venous occlusion on fibrinolytic activity in physically active and inactive men. J Appl Physiol (1985). 1994;77(5):2305-10.

70. De Paz JA, Lasierra J, Villa JG, Vilades E, Martin-Nuno MA, Gonzalez-Gallego J. Changes in the fibrinolytic system associated with physical conditioning. Eur ] Appl Physiol Occup Physiol. 1992;65(5):388-93. 
71. Kulaputana O, Macko RF, Ghiu I, Phares DA, Goldberg AP, Hagberg JM. Human gender differences in fibrinolytic responses to exercise training and their determinants. Exp Physiol. 2005;90(6):881-7.

72. de Geus EJ, Kluft C, de Bart AC, van Doornen LJ. Effects of exercise training on plasminogen activator inhibitor activity. Med Sci Sports Exerc. 1992;24(11):1210-9.

73. Gris JC, Schved JF, Feugeas O, Aguilar-Martinez P, Arnaud A, Sanchez N, et al. Impact of smoking, physical training and weight reduction on FVII, PAI-1 and hemostatic markers in sedentary men. Thromb Haemost. 1990;64(4):516-20.

74. Speiser W, Langer W, Pschaick A, Selmayr E, Ibe B, Nowacki PE, et al. Increased blood fibrinolytic activity after physical exercise: comparative study in individuals with different sporting activities and in patients after myocardial infarction taking part in a rehabilitation sports program. Thromb Res. 1988;51(5):543-55.

75. Estelles A, Aznar J, Tormo G, Sapena P, Tormo V, Espana F. Influence of a rehabilitation sports programme on the fibrinolytic activity of patients after myocardial infarction. Thromb Res. 1989;55(2):203-12.

76. Wagner DD. Cell biology of von Willebrand factor. Annu Rev Cell Biol. 1990;6:217-46.

77. Spiel AO, Gilbert JC, Jilma B. von Willebrand factor in cardiovascular disease: focus on acute coronary syndromes. Circulation. 2008;117(11):1449-59.

78. Pinsky DJ, Naka Y, Liao H, Oz MC, Wagner DD, Mayadas TN, et al. Hypoxia-induced exocytosis of endothelial cell Weibel-Palade bodies. A mechanism for rapid neutrophil recruitment after cardiac preservation. J Clin Invest. 1996;97(2):493-500.

79. El-Sayed MS, Sale C, Jones PG, Chester M. Blood hemostasis in exercise and training. Med Sci Sports Exerc. 2000;32(5):918-25.

80. van Loon JE, Sonneveld MA, Praet SF, de Maat MP, Leebeek FW. Performance related factors are the main determinants of the von Willebrand factor response to exhaustive physical exercise. PLoS One. 2014;9(3):e91687.

81. Stakiw J, Bowman M, Hegadorn C, Pruss C, Notley C, Groot E, et al. The effect of exercise on von Willebrand factor and ADAMTS-13 in individuals with type 1 and type 2B von Willebrand disease. J Thromb Haemost. 2008;6(1):90-6.

82. van Mourik JA, Boertjes R, Huisveld IA, Fijnvandraat K, Pajkrt D, van Genderen PJ, et al. von Willebrand factor propeptide in vascular disorders: $A$ tool to distinguish between acute and chronic endothelial cell perturbation. Blood. 1999;94(1):179-85.

83. Green DJ, Maiorana A, O'Driscoll G, Taylor R. Effect of exercise training on endotheliumderived nitric oxide function in humans. J Physiol. 2004;561(Pt 1):1-25.

84. Wang JS, Li YS, Chen JC, Chen YW. Effects of exercise training and deconditioning on platelet aggregation induced by alternating shear stress in men. Arterioscler Thromb Vasc Biol. 2005;25(2):454-60.

85. von Kanel R, Dimsdale JE. Effects of sympathetic activation by adrenergic infusions on hemostasis in vivo. Eur J Haematol. 2000;65(6):357-69.

86. Sadler JE. Low von Willebrand factor: sometimes a risk factor and sometimes a disease. Hematology Am Soc Hematol Educ Program. 2009:106-12.

87. Goto S, Ikeda Y, Murata M, Handa M, Takahashi E, Yoshioka A, et al. Epinephrine augments von Willebrand factor-dependent shear-induced platelet aggregation. Circulation. 1992;86(6):1859-63.

88. Tomasiak M, Stelmach H, Rusak T, Ciborowski M, Radziwon P. Vasopressin acts on platelets to generate procoagulant activity. Blood Coagul Fibrinolysis. 2008;19(7):615-24. 
89. Delp MD, O'Leary DS. Integrative control of the skeletal muscle microcirculation in the maintenance of arterial pressure during exercise. J Appl Physiol (1985). 2004;97(3):11128.

90. Small M, Tweddel AC, Rankin AC, Lowe GD, Prentice CR, Forbes CD. Blood coagulation and platelet function following maximal exercise: effects of beta-adrenoceptor blockade. Haemostasis. 1984;14(3):262-8.

91. Huizinga EG, Tsuji S, Romijn RA, Schiphorst ME, de Groot PG, Sixma J], et at. Structures of glycoprotein Ibalpha and its complex with von Willebrand factor A1 domain. Science. 2002;297(5584):1176-9.

92. Ruggeri ZM. The role of von Willebrand factor in thrombus formation. Thromb Res. 2007;120 Suppl 1:S5-9.

93. Savage B, Saldivar E, Ruggeri ZM. Initiation of platelet adhesion by arrest onto fibrinogen or translocation on von Willebrand factor. Cell. 1996;84(2):289-97.

94. Mazzeo RS. Catecholamine responses to acute and chronic exercise. Med Sci Sports Exerc. 1991;23(7):839-45.

95. Huskens D, Roest M, Remijn JA, Konings J, Kremers RM, Bloemen S, et al. Strenuous exercise induces a hyperreactive rebalanced haemostatic state that is more pronounced in men. Thromb Haemost. 2016;115(6):1109-19.

96. Ninivaggi M, de Laat M, Lance MM, Kicken CH, Pelkmans L, Bloemen S, et al. Hypoxia Induces a Prothrombotic State Independently of the Physical Activity. PLoS One. 2015;10(10):e0141797.

97. Womack CJ, Nagelkirk PR, Coughlin AM. Exercise-induced changes in coagulation and fibrinolysis in healthy populations and patients with cardiovascular disease. Sports Med. 2003;33(11):795-807.

98. Hegde SS, Goldfarb AH, Hegde S. Clotting and fibrinolytic activity change during the $1 \mathrm{~h}$ after a submaximal run. Med Sci Sports Exerc. 2001;33(6):887-92.

99. Posthuma JJ, Loeffen R, van Oerle R, Henskens YM, ten Cate H, Spronk HM, et al. Long-term strenuous exercise induces a hypercoagulable state through contact activation. Thromb Haemost. 2014;111(6):1197-9.

100. Hemker HC, Giesen P, Al Dieri R, Regnault V, de Smedt E, Wagenvoord R, et al. Calibrated automated thrombin generation measurement in clotting plasma. Pathophysiol Haemost Thromb. 2003;33(1):4-15.

101. Mosesson MW, Siebenlist KR, Meh DA. The structure and biological features of fibrinogen and fibrin. Ann N Y Acad Sci. 2001;936:11-30.

102. Ribeiro J, Almeida-Dias A, Ascensao A, Magalhaes J, Oliveira AR, Carlson J, et al. Hemostatic response to acute physical exercise in healthy adolescents. J Sci Med Sport. 2007;10(3):1649.

103. Smith JE. Effects of strenuous exercise on haemostasis. Br J Sports Med. 2003;37(5):433-5.

104. Szymanski LM, Pate RR. Effects of exercise intensity, duration, and time of day on fibrinolytic activity in physically active men. Med Sci Sports Exerc. 1994;26(9):1102-8.

105. el-Sayed MS. Effects of exercise on blood coagulation, fibrinolysis and platelet aggregation. Sports Med. 1996;22(5):282-98.

106. Leon AS, Myers MJ, Connett J. Leisure time physical activity and the 16-year risks of mortality from coronary heart disease and all-causes in the Multiple Risk Factor Intervention Trial (MRFIT). Int J Sports Med. 1997;18 Suppl 3:S208-15. 
107. McMurray RG, Ainsworth BE, Harrell JS, Griggs TR, Williams OD. Is physical activity or aerobic power more influential on reducing cardiovascular disease risk factors? Med Sci Sports Exerc. 1998;30(10):1521-9.

108. Shaper AG, Wannamethee G, Weatherall R. Physical activity and ischaemic heart disease in middle-aged British men. Br Heart J. 1991;66(5):384-94.

109. Biggs R, Mac FR, Pilling J. Observations on fibrinolysis; experimental activity produced by exercise or adrenaline. Lancet. 1947;1(6448):402-5.

110. Hansen JB, Wilsgard L, Olsen JO, Osterud B. Formation and persistence of procoagulant and fibrinolytic activities in circulation after strenuous physical exercise. Thromb Haemost. 1990;64(3):385-9.

111. Rocker L, Taenzer M, Drygas WK, Lill H, Heyduck B, Altenkirch HU. Effect of prolonged physical exercise on the fibrinolytic system. Eur J Appl Physiol Occup Physiol. 1990;60(6):47881.

112. Aird WC. Spatial and temporal dynamics of the endothelium. J Thromb Haemost. 2005;3(7):1392-406.

113. de Boer A, Kluft C, Kroon JM, Kasper FJ, Schoemaker HC, Pruis J, et al. Liver blood flow as a major determinant of the clearance of recombinant human tissue-type plasminogen activator. Thromb Haemost. 1992;67(1):83-7.

114. Chandler WL, Levy WC, Veith RC, Stratton JR. A kinetic model of the circulatory regulation of tissue plasminogen activator during exercise, epinephrine infusion, and endurance training. Blood. 1993;81(12):3293-302.

115. Nofer JR, Walter M, Kehrel B, Wierwille S, Tepel M, Seedorf U, et al. HDL3-mediated inhibition of thrombin-induced platelet aggregation and fibrinogen binding occurs via decreased production of phosphoinositide-derived second messengers 1,2-diacylglycerol and inositol 1,4,5-tris-phosphate. Arterioscler Thromb Vasc Biol. 1998;18(6):861-9.

116. Barlow CE, Defina LF, Radford NB, Berry JD, Cooper KH, Haskell WL, et al. Cardiorespiratory fitness and long-term survival in "low-risk" adults. J Am Heart Assoc. 2012;1(4):e001354.

117. Kjaer M, Secher NH, Galbo H. Physical stress and catecholamine release. Bailliere's clinical endocrinology and metabolism. 1987;1(2):279-98.

118. Cadroy Y, Pillard F, Sakariassen KS, Thalamas C, Boneu B, Riviere D. Strenuous but not moderate exercise increases the thrombotic tendency in healthy sedentary male volunteers. J Appl Physiol (1985). 2002;93(3):829-33.

119. Chen YW, Apostolakis S, Lip GY. Exercise-induced changes in inflammatory processes: Implications for thrombogenesis in cardiovascular disease. Ann Med. 2014;46(7):439-55.

120. Kargotich S, Goodman C, Keast D, Morton AR. The influence of exercise-induced plasma volume changes on the interpretation of biochemical parameters used for monitoring exercise, training and sport. Sports Med. 1998;26(2):101-17.

121. Andrew M, Carter C, O'Brodovich H, Heigenhauser G. Increases in factor VIII complex and fibrinolytic activity are dependent on exercise intensity. J Appl Physiol (1985). 1986;60(6):1917-22.

122. Cohen RJ, Epstein SE, Cohen LS, Dennis LH. Alterations of fibrinolysis and blood coagulation induced by exercise, and the role of beta-adrenergic-receptor stimulation. Lancet. 1968;2(7581):1264-6.

123. latridis SG, Ferguson JH. Effect of physical exercise on blood clotting and fibrinolysis. J Appl Physiol. 1963;18:337-44. 
124. Bourey RE, Santoro SA. Interactions of exercise, coagulation, platelets, and fibrinolysis--a brief review. Med Sci Sports Exerc. 1988;20(5):439-46.

125. Gawel MJ, Glover V, Burkitt M, Sandler M, Rose FC. The specific activity of platelet monoamine oxidase varies with platelet count during severe exercise and noradrenaline infusion. Psychopharmacology (Berl). 1981;72(3):275-7.

126. Haber P, Silberbauer K, Sinzinger H. [Quantitative studies on reversible thrombocyte aggregation during exertion]. Schweiz Med Wochenschr. 1980;110(41):1488-91.

127. Rakobowchuk M, McGowan CL, de Groot PC, Hartman JW, Phillips SM, MacDonald MJ. Endothelial function of young healthy males following whole body resistance training. J Appl Physiol (1985). 2005;98(6):2185-90.

128. Hilberg T, Nowacki PE, Muller-Berghaus G, Gabriel HH. Changes in blood coagulation and fibrinolysis associated with maximal exercise and physical conditioning in women taking low dose oral contraceptives. J Sci Med Sport. 2000;3(4):383-90.

129. Kahraman S, Bediz CS, Piskin O, Aksu I, Topcu A, Yuksel F, et al. The effect of the acute submaximal exercise on thrombin activatable fibrinolysis inhibitor levels in young sedentary males. Clin Appl Thromb Hemost. 2011;17(4):414-20.

130. Sand KL, Flatebo T, Andersen MB, Maghazachi AA. Effects of exercise on leukocytosis and blood hemostasis in 800 healthy young females and males. World J Exp Med. 2013;3(1):1120. 


\section{SUPPLEMENTAL DATA}

\section{Supplemental Methods}

\section{Study population}

Five physically fit (non-professional) male cyclists were included in the pilot study and cycled $80 \mathrm{~km}$ on each of three consecutive days. All participants were non-smokers, none had a known haemostatic or cardiovascular disease and none used anti-platelet/ anti-coagulant drugs for one week prior to the study. All participants gave written informed consent before the study and blood withdrawal was approved by the medical research ethics committee of Maastricht University Medical Centre.

\section{Repetitive exercise}

On each of three subsequent days, the participants cycled about $80 \mathrm{~km}$ over the course of 4 hours, always at the same time of day to take into account possible diurnal effects. ${ }^{1}$ Submaximal exercise intensity was achieved by cycling in a hilly landscape, covering a total of 800 height meters, with a maximum slope of $10 \%$, inducing $90-95 \%$ intensity for approximately 10 minutes when climbing hills and $>75 \%$ intensity between hills.

\section{Blood sample collection}

Blood samples were collected before and immediately after exercise on each of the three study days. Venous blood was collected in $3.2 \%(w / v)$ citrated Vacutainer tubes (Becton Dickinson (BD) Vacutainer System).

\section{Analytical methods}

To characterize changes in their haemostatic profile, we measured VWF (total antigen ${ }^{2}$, VWF propeptide ${ }^{3}$ and active VWF) levels, $\mathrm{FVIII}^{2,4}$, thrombin generation ${ }^{5}$, fibrinogen levels ${ }^{4}$, platelet activation ${ }^{6}$ and clot lysis time ${ }^{4}$ (methods according to references). Peripheral blood cell counts in citrated whole blood were determined using a COULTER counter® analyser (Beckman Coulter, Woerden, the Netherlands).

VWF:Ag and active VWF were measured in plasma by enzyme linked immunosorbent assays (ELISAs). The active VWF assay is based on a llama-derived variable heavy chain (VHH) directed against the $A 1$ domain of VWF, which is only exposed upon unfolding of VWF. 'Briefly, 96 wells microtiter plates (NUNC Maxisorp, Thermo Fisher Scientific, USA) were coated overnight at $4^{\circ} \mathrm{C}$ with VHH against active VWF or with polyclonal antibody against human VWF (A0082, Dako, Denmark) (VWF:Ag) and blocked with $2 \%$ bovine serum albumin (BSA, Sigma, USA) in phosphate-buffered saline (PBS) for 45 minutes at room temperature. After extensive washing with $0.01 \%$ Tween-20 in PBS, plasma samples (diluted in $\mathrm{PBS} / 1 \% \mathrm{BSA}$ ) were incubated for 2 hours at room temperature. 
Following another round of washing the wells were incubated with HRP-conjugated polyclonal anti-VWF (P0226, Dako, Denmark) in PBS/1\% BSA for 2 hours at room temperature. Plates were then washed three times more before measuring the binding of active VWF to the VHH or VWF:Ag to the polyclonal anti-VWF antibodies by using SIGMAFAST OPD (Sigma, USA) as a substrate for HRP. The substrate reaction was stopped with $3 \mathrm{M}$ sulfuric acid $\left(\mathrm{H}_{2} \mathrm{SO}_{4}\right.$ Aldrich, USA). Optical densities (OD) were measured at $490 \mathrm{~nm}$ using an ELx808 Absorbance Microplate Reader (Biotek, USA). Normal pooled plasma (NPP) was used as a standard in every plate and plasma sample results were normalized (\%) to NPP on the same plate.

\section{REFERENCES}

1. Zadow EK, Kitic CM, Wu SSK, Fell JW, Adams MJ. Time of day and short-duration highintensity exercise influences on coagulation and fibrinolysis. Eur ] Sport Sci. 2018:1-9.

2. Ninivaggi $M$, de Laat $M$, Lance $M M$, Kicken $C H$, Pelkmans $L$, Bloemen $S$, et al. Hypoxia Induces a Prothrombotic State Independently of the Physical Activity. PLoS One. 2015;10(10):e0141797.

3. van Mourik JA, Boertjes R, Huisveld IA, Fijnvandraat K, Pajkrt D, van Genderen PJ, et al. von Willebrand factor propeptide in vascular disorders: $A$ tool to distinguish between acute and chronic endothelial cell perturbation. Blood. 1999;94(1):179-85.

4. Huskens D, Roest M, Remijn JA, Konings J, Kremers RM, Bloemen S, et al. Strenuous exercise induces a hyperreactive rebalanced haemostatic state that is more pronounced in men. Thromb Haemost. 2016;115(6):1109-19.

5. Bloemen S, Huskens D, Konings J, Kremers RM, Miszta A, de Laat B, et al. Interindividual Variability and Normal Ranges of Whole Blood and Plasma Thrombin Generation. Journal of Applied Laboratory Medicine. 2017;2(2).

6. Huskens D, Sang Y, Konings J, van der Vorm L, de Laat B, Kelchtermans H, et al. Standardization and reference ranges for whole blood platelet function measurements using a flow cytometric platelet activation test. PLoS One. 2018;13(2):e0192079.

7. Hulstein JJ, de Groot PG, Silence K, Veyradier A, Fijnheer R, Lenting PJ. A novel nanobody that detects the gain-of-function phenotype of von Willebrand factor in ADAMTS13 deficiency and von Willebrand disease type 2B. Blood. 2005;106(9):3035-42. 


$$
\text { Chapter } 5
$$




\title{
A hypoxic environment attenuates exercise-induced procoagulant changes due to decreased platelet activation
}

\author{
Kicken $\mathrm{CH}$, van der Vorm LN, Zwaveling $\mathrm{S}$, \\ Schoenmaker E, Remijn JA, Huskens D, \\ de Laat $B$
}

TH Open. 2019;3:e216-e226 


\section{ABSTRACT}

Introduction - Although physical exercise is protective against cardiovascular disease, it can also provoke sudden cardiac death ('exercise paradox'). Epidemiological studies suggest that systemic hypoxia at high altitude is a risk factor for venous thromboembolism (VTE). Forthcoming, this study investigated the effect of repeated exercise at high altitude on blood coagulation, platelet function and fibrinolysis.

Methods - Six trained male volunteers were recruited. Participants ascended from sea level to $3,375 \mathrm{~m}$ altitude. They performed four exercise tests at $65-80 \%$ of their heart rate reserve for 2 hours: one time at sea level and three times on consecutive days at 3,375 $\mathrm{m}$ altitude. Thrombin generation (TG) was measured in whole blood, platelet-rich and platelet-poor plasma. Coagulation factor levels were measured. Platelet activation was measured as $\alpha$ llb $\beta 3$ activation and P-selectin expression. Fibrinolysis was studied using a clot-lysis assay.

Results - Normoxic exercise increased plasma Peak TG through increased FVIII, and increased VWF and active VWF levels. Platelet granule release potential was slightly decreased. After repetitive hypoxic exercise, the increase in (active) VWF tapered, and there was no more distinct exercise-related increase in Peak. Platelet aggregation potential and platelet-dependent TG decreased at high altitude. There were no effects on fibrinolysis upon exercise and/or hypoxia.

Conclusion - Strenuous exercise induces a procoagulant state that is mediated by the endothelium, by increasing VWF and secondarily raising FVIII levels. After repetitive exercise, the amplitude of the endothelial response to exercise diminishes. A hypoxic environment appears to further attenuate the procoagulant changes by decreasing platelet activation and platelet-dependent TG. 


\section{INTRODUCTION}

Mountaineering involves repetitive physical exercise in a hypoxic environment. Although physical exercise is generally protective against cardiovascular events, there are numerous reports of exercise-related thromboembolic and cardiovascular events. ${ }^{1-3}$ Additionally, it has been found that high altitude increases the risk of venous thromboembolism (VTE)..$^{4-7}$ Moreover, cardiac arrest at high altitude due to coronary thrombosis has been reported. ${ }^{8}$

The risk of cardiovascular and thromboembolic events is partially determined by hypercoagulability. Exercise is known to exert many effects on the haemostatic system, mainly through endothelial activation, which elevates von Willebrand factor (VWF) and factor VIII (FVIII), platelet hyperreactivity, increased thrombin generation (TG) as well as elevated fibrinolytic markers. ${ }^{9-11}$ All in all, these changes result in a shift towards a transient prothrombotic state. ${ }^{12}$ The influence of hypoxia on haemostasis is less well characterized. A few studies found that systemic hypoxia influences haemostasis through elevation of factor FVIII levels, as is occurring in response to strenuous exercise. ${ }^{13,14}$ It has long been known that elevated FVIII levels are a risk factor for VTE, likely by increasing TG. Mechanistically, hypoxia may induce this FVIII-dependent increase in TG via alteration of the redox status of the blood, i.e. by inducing reactive oxygen species (ROS) formation. ${ }^{15}$ Supporting this, the anticoagulant vitamin E prevented increases in both FVIII and TG following 2 hours of exposure to normobaric hypoxia. ${ }^{14}$

Because both hypoxia and exercise induce hypercoagulability, it seems likely that exercise amplifies the altitude-induced hypercoagulability. However, several studies found that hypoxia actually attenuates the exercise-induced hypercoagulable response, mainly through the depression of platelet activation. ${ }^{16-19}$ It has never been investigated whether this effect persists after repeated exercise at high altitude. Forthcoming, this pilot study aimed to investigate the effect of repeated cycling at 3,375 m altitude on thrombin generation, platelet activation and fibrinolysis. 


\section{METHODS}

\section{Inclusion of subjects}

This study was approved by the medical research ethics committee from Maastricht University (METC azM/UM, reference NL61217.068.17), was monitored by the Clinical Trial Center Maastricht and met all standards of the declaration of Helsinki (version 10, 2013). The primary endpoint was whole blood TG peak height at high altitude. Group size was calculated based on data from a previous high altitude study, ${ }^{20}$ in which peak height was $139 \mathrm{nM}$ at sea level and rose to $241 \mathrm{nM}$ at 2,045 $\mathrm{m}$ altitude, estimated standard deviation $40 \mathrm{nM}$. With 8 pairwise comparisons and $\alpha=0.05$, at least 4 subjects needed to be recruited to achieve a power of $80 \% .{ }^{21}$ To allow dropouts without underpowering the study, a total of 6 trained and healthy men were included in this study. Exclusion criteria were cardiovascular disease, pulmonary disease, impaired mobility, known coagulation disorders and medication interfering with coagulation (heparins, vitamin $\mathrm{K}$ antagonists, new oral anticoagulants or NSAIDs). After informed consent, but before inclusion, all participants passed a medical assessment by an independent cardiologist, consisting of history taking, vital signs (peripheral oxygen saturation $\left(\mathrm{SpO}_{2}\right)$, heart rate, blood pressure), auscultation of heart and lungs, and an exhaustive ergometry test according to the Bruce protocol. ${ }^{22}$ Prior to the study, all participants were exercising ( 4 out of 6 cycling, subject 3 fitness/running, subject 5 hockey) on a regular basis, for an average of 5.6 hours per week (SD 1.3 hours).

\section{Exercise and altitude protocol}

The altitude and exercise protocol is shown in Figure 1. Vigorous exercise can be defined as $60-85 \%$ of heart rate reserve (HRR), where $H R R=H$ max $-H R_{\text {rest. }}{ }^{23}$ These boundaries were calculated using data from the exhaustive ergometry test, using the following equation: exercise $H R=H R_{\text {rest }}+0.6 / 0.85 \times H R R$. The participants delivered monitored physical exercise by cycling for 2 hours on a racing bike mounted on a stationary frame (Tacx Blue Twist, Wassenaar, the Netherlands). Heart rate was monitored every 10 minutes using a chest strap (Polar FT1 sports watch, Kempele, Finland), and participants were encouraged to keep their HR between their predefined HRR boundaries to ensure adequate exercise intensity. The exercise test was performed on 4 different occasions: one time at $50 \mathrm{~m}$ altitude (normoxic exercise), and three times on three consecutive days at 3,375 $\mathrm{m}$ altitude (hypoxic exercise). Between the baseline measurement and the hypoxic exercise tests, participants acclimatized to the altitude. First, they stayed for three days at 2,473 m altitude, where daily activities but not exercise were allowed. On the third day, they ascended further to 3,375 m altitude. After one night at 3,375 $\mathrm{m}$ altitude (day four), the first hypoxic exercise tests were performed, followed by the second and third hypoxic exercise test on the next two days (day five and six). The test 
room and laboratory were set up in a ventilated mountain cabin at room temperature. Participants stayed overnight at 3,375 $\mathrm{m}$ altitude unless there was a reason for earlier descent. On day 6 , after completion of the last exercise test at 3,375 m altitude, the participants descended immediately to $1,224 \mathrm{~m}$ altitude.

\section{Lake Louise Acute Mountain Sickness questionnaire}

To record signs of acute mountain sickness (AMS), participants filled out a questionnaire based on the Lake Louise Consensus on the Definition of Altitude Illness ${ }^{24}$ at $50 \mathrm{~m}$ altitude, and every morning at 2,473 $\mathrm{m}$ and 3,375 $\mathrm{m}$ altitude.

\section{Blood collection}

Blood was drawn before and after every exercise test by venipuncture of the antecubital vein. The blood was aseptically drawn in Vacutainer tubes (Greiner Bio-One) containing $3.2 \%$ sodium citrate (9 volumes blood, 1 volume anticoagulant). To inhibit contact activation, a separate $3 \mathrm{ml}$ citrate tube with added corn trypsin inhibitor (CTI, Enzyme research laboratories, USA; final concentration $50 \mu \mathrm{g} / \mathrm{ml}$ ) was drawn. Any particularities (bleeding, bruising, difficult puncture, and haemolysis) were noted. The blood was kept at room temperature $\left(21^{\circ} \mathrm{C}\right)$ until use. Platelet-rich plasma (PRP) was obtained by centrifuging the blood at $240 \mathrm{~kg}$ for 15 minutes. Platelet-poor plasma (PPP) was obtained by centrifuging the blood twice at $2830 \mathrm{~kg}$ for 10 minutes and immediately placed on dry ice $\left(-80^{\circ} \mathrm{C}\right)$ for later analysis.

\section{Biochemical markers}

Albumin as a marker for dehydration, creatinine and urea as markers for kidney function, lactate as a marker for anaerobic metabolism and C-reactive protein as a marker for inflammation were measured with an ARCHITECT ci8200 (Abbott Diagnostics, Lake Forest, USA).

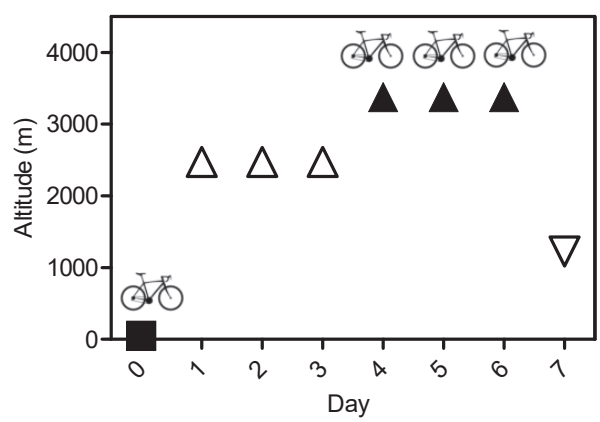

Figure 1. Schematic representation of the study set-up. Participants performed an exercise test on 4 occasions: one time at $50 \mathrm{~m}$ altitude (normoxic exercise), and three times on three consecutive days at $3,375 \mathrm{~m}$ altitude (hypoxic exercise). Before the hypoxic exercise tests, participants acclimatized to the altitude for 3 days at 2,475 m altitude. After the last hypoxic exercise test, participants descended to $1,225 \mathrm{~m}$ altitude. 


\section{Blood count}

Red blood cell count (RBC, 10 $12 / \mathrm{L})$, haematocrit ( $\mathrm{Ht}, \mathrm{L} / \mathrm{L})$, haemoglobin levels $(\mathrm{Hb}$, $\mathrm{mmol} / \mathrm{L}$ ), mean corpuscular haemoglobin concentration (MCHC, mmol/L), white blood cell count (WBC, $\left.\cdot 10^{9} / \mathrm{L}\right)$, granulocyte count $\left(\mathrm{GR}, 10^{9} / \mathrm{L}\right)$, lymphocyte count $\left(\mathrm{LY}, 10^{9} / \mathrm{L}\right)$, monocyte count $\left(\mathrm{MO}, 10^{\circ} / \mathrm{L}\right)$ and platelet count $\left(\mathrm{PC}, 10^{\circ} / \mathrm{L}\right)$ were determined with a Coulter LH-750 analyser (Beckman Coulter, Brea, USA) within 2 hours after venipuncture in the citrated whole blood. Values were corrected for $10 \%$ dilution by the citrate solution present in blood tubes.

\section{Coagulation factor analysis}

Antithrombin (AT), factor VIII concentration (FVIII:C), von Willebrand Factor antigen (VWF:Ag) and fibrinogen levels were measured by batch analysis in PPP by the STA-R Evolution (Diagnostica Stago, Leiden, the Netherlands). Active VWF was measured in plasma by an enzyme-linked immunosorbent assay (ELISA), as described previously. ${ }^{25}$ Normal pooled plasma (NPP) was used as a standard in every plate and sample results were normalized (\%) to NPP on the same plate.

\section{Whole blood thrombin generation}

The whole blood thrombin generation assay was performed within 2 hours after blood collection, triggered with $0.5 \mathrm{pM}$ tissue factor (TF) or without added TF. The method has been described previously. ${ }^{26}$ For the high altitude measurements, the necessary equipment was installed at 3,375 m altitude in a ventilated room, to allow measurement in fresh undisturbed blood. In short, $30 \mu \mathrm{l}$ citrated whole blood (WB) was mixed with $10 \mu \mathrm{l}$ rhodamine substrate (thrombin specific fluorogenic substrate, $1.8 \mathrm{mM}$ ) and activated with $20 \mu \mathrm{l}$ of either a mix of $\mathrm{CaCl}_{2}(50 \mathrm{mM})$ and HEPES buffer containing 5 $\mathrm{mg} / \mathrm{ml}$ BSA (BSA5) with/without TF, or calibrator ( $\alpha_{2}$ macroglobulin-thrombin complex, in-house prepared, $300 \mathrm{nM}$ thrombin activity). The sample was mixed, and $5 \mu \mathrm{l}$ was transferred immediately onto a paper disk (Whatman $\mathrm{GmbH}$, Dassel, Germany) in Immulon $2 \mathrm{HB}$ flat-bottom 96-well plates (Thermo Scientific, Waltham, USA), and covered with $40 \mu \mathrm{l}$ mineral oil (USB Corporation). Thrombin generation was measured in triplicate in a 96-well plate fluorometer (Ascent reader, Thermolabsystems OY, Helsinki Finland) equipped with a $485 / 538 \mathrm{~nm}$ filter set (excitation/emission). Samples were measured for 40 minutes at $37^{\circ} \mathrm{C}$. Raw data were converted into thrombograms as described previously. ${ }^{27}$ Parameters derived from the TG curve were peak height (Peak, nM) and endogenous thrombin potential (ETP, nM.min).

\section{Calibrated automated thrombinography in plasma}

The calibrated automated thrombinography assay (CAT) was performed on the spot in PRP and later by batch analysis in snap-frozen PPP. The method has been described 
previously. ${ }^{28}$ For the measurements of PRP TG at high altitude, the necessary equipment was installed at 3,375 $\mathrm{m}$ altitude in a ventilated room. TG was determined in triplicate at $37^{\circ} \mathrm{C}$ in PRP after the addition of 1 pM TF (PRP-reagent, Thrombinoscope, Maastricht, the Netherlands). TG was also determined in triplicate at $37^{\circ} \mathrm{C}$ in PPP after addition of 1 pM TF plus $4 \mu \mathrm{mol} / \mathrm{L}$ phospholipids (PPP-low reagent), in PPP after addition of 5 pM TF plus $4 \mu \mathrm{mol} / \mathrm{L}$ phospholipids (PPP-reagent), and in citrate-CTI anticoagulated PPP (PPPlow reagent), according to the manufacturer's instructions. A sample of NPP was added on each 96-wells plate for normalization, to obtain acceptable inter-assay variations. ${ }^{29}$ As a calibrator, $\alpha_{2}$ macroglobulin-thrombin complex $\left(\alpha_{2} \mathrm{M}-\mathrm{T}, \pm 600 \mathrm{nM}\right.$ thrombin activity, Thrombinoscope, Maastricht, the Netherlands) was used. Z-Gly-Gly-Arg-AMC (FluCa kit, Thrombinoscope, Maastricht the Netherlands) was used as fluorogenic substrate. The thrombograms were measured in the fluorometer equipped with a $390 / 460 \mathrm{~nm}$ filter set (excitation/emission) and a dispenser. Immulon 2HB, round-bottom 96-well plates (Thermo Scientific, Waltham, USA) were used. A dedicated software program (Thrombinoscope, Maastricht, the Netherlands) calculated the thrombograms. Parameters derived from the TG curve were Peak (\% of NPP) and ETP (\% of NPP).

\section{Platelet activation test in whole blood}

The flow-cytometric platelet activation test in WB (PACT) was performed as described previously. ${ }^{30}$ Thrombin receptor activator peptide (TRAP-6, final concentration $30 \mu \mathrm{M}$, SFLLRN, H-2936; Bachem, Germany), and collagen-related peptide (CRP, final concentration $5 \mu \mathrm{g} / \mathrm{ml}$, a kind gift of Prof. Farndale, University of Cambridge, UK) were used as platelet agonists. Moreover, an unstimulated control condition without agonist was included. The reaction mixtures contained three antibodies: APC-conjugated CD42b, (BD Bioscience), PE-conjugated anti-P-selectin and FITC-conjugated PAC-1 against activated $\alpha$ llb $\beta 3$ (BD Pharmingen, Franklin Lakes, USA). WB was preheated at $37^{\circ} \mathrm{C}$ for $10 \mathrm{~min}$ and the tests were performed at $37^{\circ} \mathrm{C}$. WB was diluted $1: 4$ in HBS and $5 \mu \mathrm{l}$ of this diluted blood were added to each reaction mixture. After exactly $20 \mathrm{~min}$ of incubation at $37^{\circ} \mathrm{C}$, reactions were stopped by adding $250 \mu \mathrm{l}$ fixation solution $(137 \mathrm{mmol} / \mathrm{L} \mathrm{NaCl}$, $2.7 \mathrm{mmol} / \mathrm{L} \mathrm{KCl}, 1.12 \mathrm{mmol} / \mathrm{L} \mathrm{NaH}_{2} \mathrm{PO}_{4} 1.15 \mathrm{mmol} / \mathrm{L} \mathrm{KH_{2 }} \mathrm{PO}_{4}, 10.2 \mathrm{mmol} / \mathrm{L} \mathrm{Na}_{2} \mathrm{HPO}_{4} 4$ $\mathrm{mmol} / \mathrm{L}$ EDTA, $0.5 \%$ formaldehyde). Flow cytometry was used to discriminate platelets from other cells, using the forward and sideward scatter pattern and by gating on the CD42b positive cells. Fluorescent intensity in the FITC gate and PE gate was selected to determine activated $\alpha \mathrm{llb} \beta 3$ and P-selectin density, respectively, and results were expressed as median fluorescent intensity (MFI).

\section{Clot lysis assay}

To examine fibrinolysis, a turbidimetric clot lysis assay was performed in PPP using tissue-plasminogen activator (t-PA, Actilyse, Boehringer Ingelheim, Germany). Samples 
were tested in duplo and preheated at $37^{\circ} \mathrm{C}$ for 10 minutes. Eighty $\mu$ of PPP was spiked with $20 \mu$ l of a TF/BSA5 solution (final concentration 1 pM) plus phospholipids (final concentration $4 \mu \mathrm{mol} / \mathrm{L}$ ). Fibrin clot formation was started by adding $20 \mu \mathrm{l}$ of a preheated $\mathrm{CaCl}_{2} / \mathrm{BSA} 5$ solution (final concentration $16.7 \mathrm{mM}$ ) with t-PA (final concentration 100 IU). Optical density was measured at $405 \mathrm{~nm}$ with 20 s intervals for 1 hour at $37^{\circ} \mathrm{C}$ using a plate reader (SpectraMax M2, Molecular Devices, USA). All samples from 1 subject were measured simultaneously to avoid inter-assay error. Clot lysis time (CLT, min) was defined as the time from half-maximal fibrin formation to half-maximal degradation.

\section{Data analysis}

Statistical analyses and figures were generated using Prism version 7 (GraphPad Software Inc., La Jolla, USA). Generally, data are represented as median \pm interquartile range or [ $25^{\text {th }} ; 75^{\text {th }}$ percentile]. Descriptive statistics were used to discover trends in the data. The data presented in Figures 3, 4, 5 and 6 and Tables S2 and S3 are expressed as the absolute change compared to the first measurement at sea level; a value above 0 indicates an increase, a value below 0 indicates a decrease. Absolute change, not percentage change, was chosen because this is more statistically powerful. ${ }^{31}$ For statistical analysis, a nonparametric distribution was assumed because of the small sample size. The Wilcoxon signed ranks test was used to determine if the data presented in Figures 3, 4, 5 and 6 and Tables S2 and S3 were different from zero. Friedman's test with Dunn's posthoc analysis was used to determine the overall statistical significance of changes within participants. A p-value $<0.05$ was considered significant.

\section{RESULTS}

Six trained adult men were recruited and passed the medical check-up (median age 33.5 years, range $18-48$ years). All six participants completed the full study protocol. None of the participants experienced signs of acute mountain sickness during the duration of the study (maximum LLO score 3 points). As shown in Figure 1, the participants performed monitored exercise for 2 hours on 4 occasions: once at $50 \mathrm{~m}$ altitude, and three times at 3,375 $\mathrm{m}$ altitude. The participants cycled at an adequate exercise intensity for the majority of the time (Figure S1).

\section{Vital signs, biochemical markers, blood count, and coagulation factor levels}

Results for vital signs are shown in Table $\mathrm{S} 1$ section $\mathrm{A}$. The $\mathrm{SpO}_{2}$ dropped from $99 \%$ to $93 \%$ at day 1 on $3,375 \mathrm{~m}$ altitude, and to $92 \%$ on day 2 and 3, respectively. The resting heart rate increased slightly, from 56 to $64 \mathrm{~min}^{-1}$ at day 1 on $3,375 \mathrm{~m}$ altitude. 
Biochemical marker results are shown in Table S1 section B. Exposure to high altitude increased lactate levels slightly, yet non-significantly, and this effect was similar to exercise at sea level. Lactate levels did not rise any further due to the hypoxic exercise tests. Both normoxic and hypoxic exercise slightly increased the creatinine level, although it only rose significantly after the normoxic exercise. Urea, albumin and C-reactive protein levels did not change compared to baseline.

Blood count results are shown in Table $\mathrm{S} 1$ section $\mathrm{C}$. Hb levels increased after acclimatization to high altitude, with a concurrent elevation in $\mathrm{MCHC}$ that was most prominent on day 2 at 3,375 m. Altitude itself did not affect total WBC. RBC and Ht did not change due to the exercise tests or due to high altitude, except for day 2 pre-exercise. There was a consistent exercise-induced increase in total WBC, that depended on an increase in granulocyte count, not lymphocyte or monocyte counts. This effect was present after both the normoxic and hypoxic exercise tests. The WBC returned to baseline levels every day. Altitude and/or exercise did not have a significant effect on platelet count, although there appeared to be a trend towards increased platelet count after each exercise test.

Coagulation factor level results are shown in Table S1 section D. Altitude itself caused a small, non-significant increase in baseline FVIII levels, without an increase in VWF or active VWF levels. The absolute change of VWF, active VWF and FVIII compared to $50 \mathrm{~m}$ pre-exercise is shown in Figure 2. Repeated exercise caused a distinct zig-zag pattern in VWF, active VWF and FVIII levels. VWF increased due to exercise, an effect that was most outspoken at sea level, and was less pronounced at day 2 and 3 at 3,375 m. VWF levels returned to baseline every day. Active VWF increased as well due to the exercise. The active VWF response was tapered after repeated hypoxic exercise. Both normoxic and hypoxic exercise increased the FVIII level, and FVIII did not fully return to baseline levels during the three days at high altitude. Fibrinogen and antithrombin levels were neither affected by exercise, nor by high altitude.

\section{Effect of normoxic exercise on TG, platelet activation and clot lysis time}

Results are expressed as the absolute change compared to $50 \mathrm{~m}$ pre-exercise; a value above 0 indicates an increase, a value below 0 indicates a decrease. Effects of normoxic and hypoxic exercise on TG in WB and PRP are shown in Figure 3, and on TG in PPP are shown in Figure 4. The absolute values can be found in Table S2. There was no effect of normoxic exercise on WB TG Peak ( 0 pM TF: $p=0.44 ; 0.5$ pM TF: $p=0.81$, compared to zero), nor on ETP (0 pM TF: $p>0.99 ; 0.5$ pM TF: $p=0.63$, compared to zero). In both PPP and PRP, normoxic exercise increased the Peak (PPP CTI-1 pM TF: $p=0.03 ; 5$ pM TF: $p=0.03$; PRP: $p=0.03$ compared to zero), but did not change the ETP (PPP 1 pM TF: $p>0.99$; CTI-1 pM TF: $p=0.06 ; 5 p M$ TF: $p=0.44 ;$ PRP: $p=0.22$, compared to zero). 

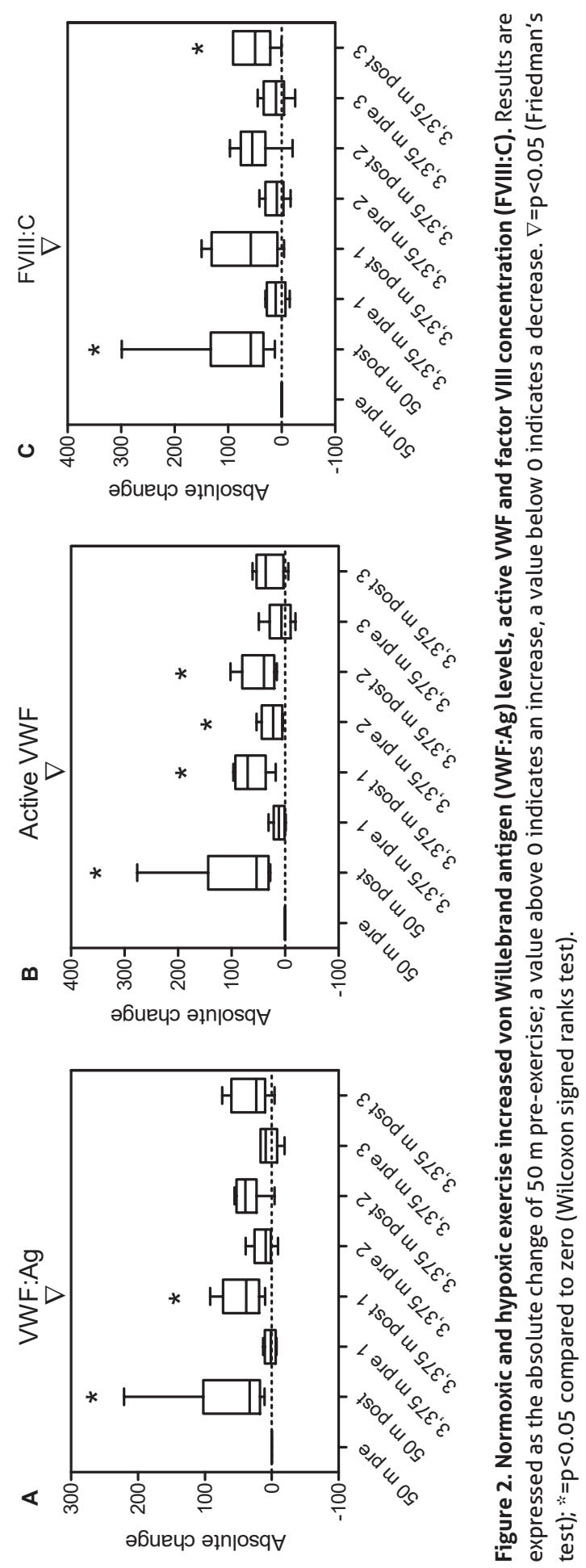

는 

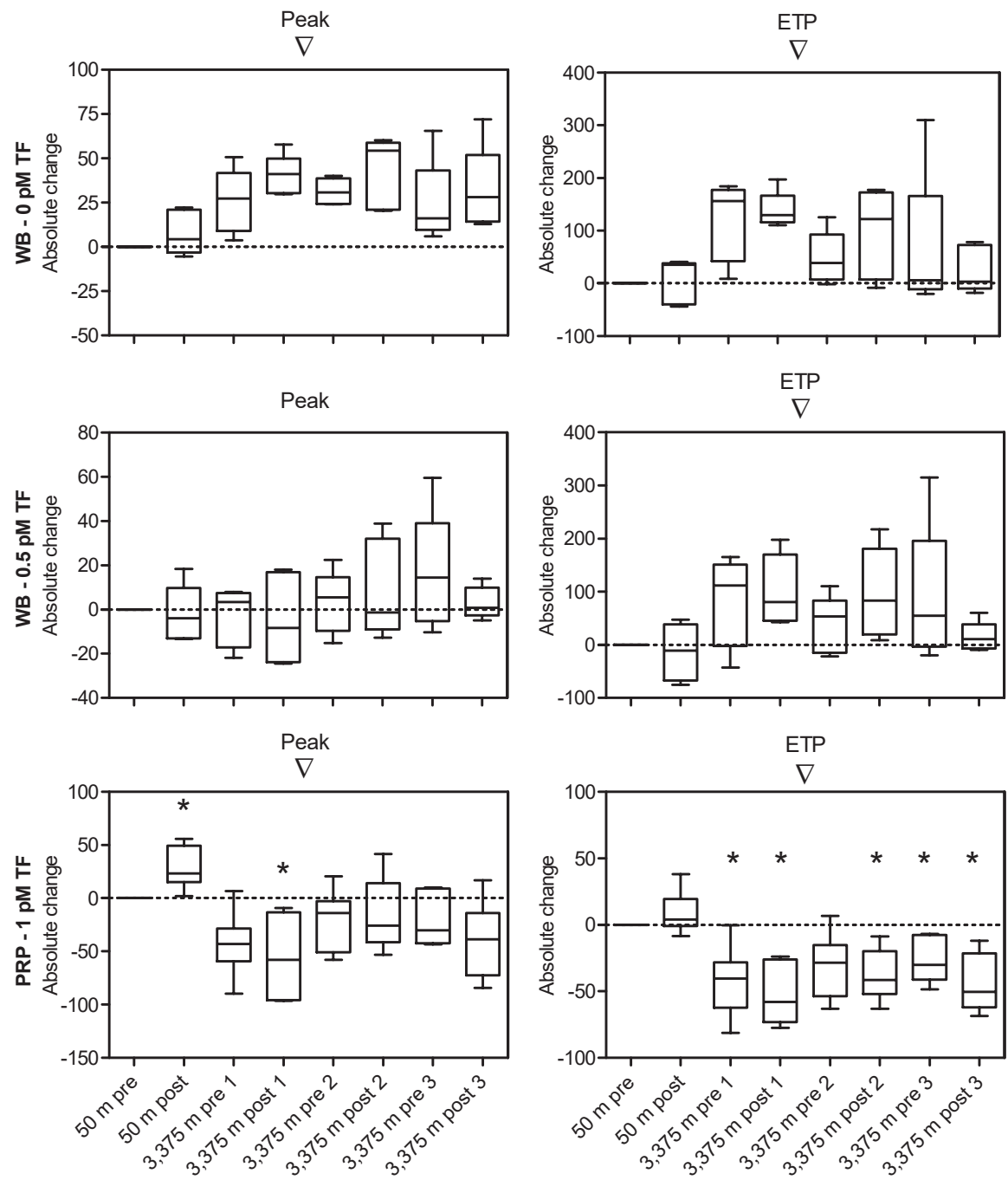

Figure 3. Influence of normoxic and hypoxic exercise on thrombin generation (TG) in whole blood (WB) and platelet-rich plasma (PRP). TG was measured before and after 2 hours of strenuous exercise, once at $50 \mathrm{~m}$ altitude and three times at $3,375 \mathrm{~m}$ altitude, in whole blood (WB) and PRP. Parameters derived from the TG curve are peak height (Peak in $\mathrm{nM}$ or \% of NPP) and endogenous thrombin potential (ETP in $\mathrm{nM} \cdot \min$ or \% of NPP). $\mathrm{N}=6,1$ measurement is missing (subject 5, WB TG post 3). Results are expressed as the absolute change of $50 \mathrm{~m}$ pre-exercise; a value above 0 indicates an increase, a value below 0 indicates a decrease. $\nabla=p<0.05$ (Friedman's test); * $=p<0.05$ compared to zero (Wilcoxon signed ranks test). 
Peak
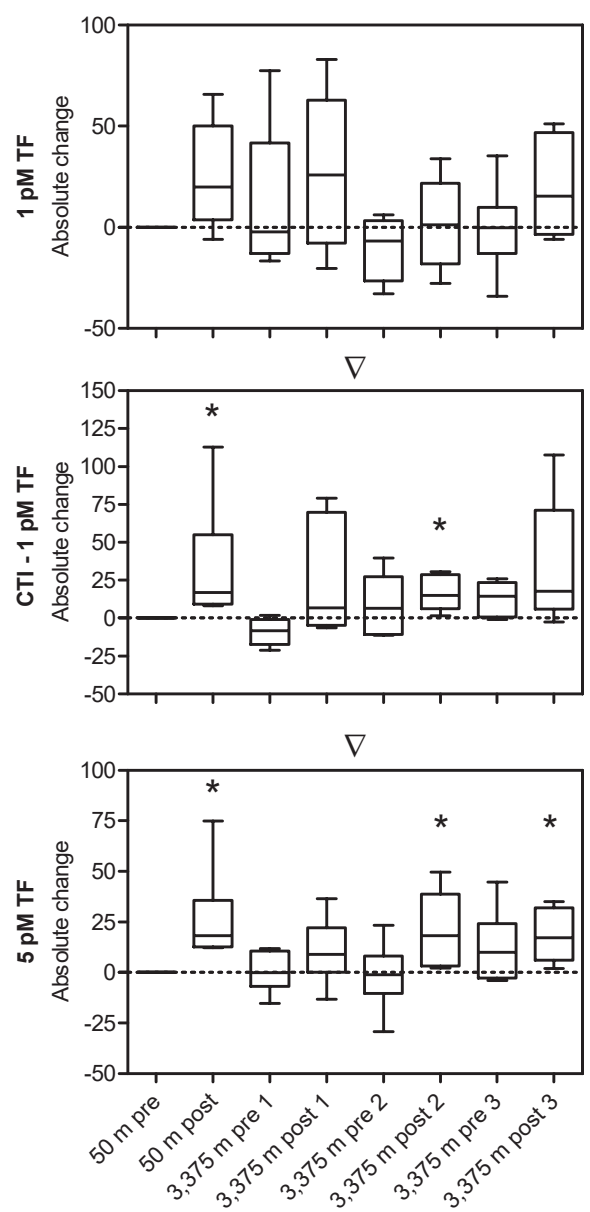

ETP
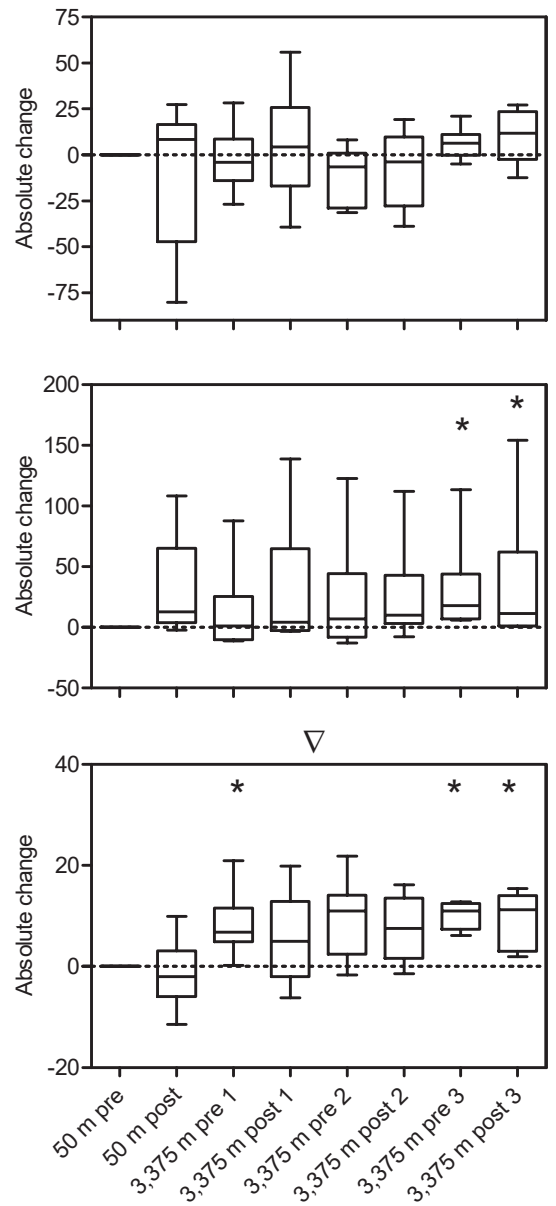

Figure 4. Influence of normoxic and hypoxic exercise on thrombin generation (TG) in platelet-poor plasma (PPP). TG was measured before and after 2 hours of strenuous exercise, once at $50 \mathrm{~m}$ altitude and three times at 3,375 m altitude, in PPP with and without added corn trypsin inhibitor (CTI). Parameters derived from the TG curve are peak height (Peak \% of NPP) and endogenous thrombin potential (ETP in \% of NPP). $N=6$, results are expressed as the absolute change of $50 \mathrm{~m}$ pre-exercise; a value above 0 indicates an increase, a value below 0 indicates a decrease. $\nabla=p<0.05$ (Friedman's test); * $=p<0.05$ compared to zero (Wilcoxon signed ranks test).

The effects of normoxic and hypoxic exercise on platelet activation are shown in Figure 5. The absolute values can be found in Table S3. Baseline (unstimulated) platelet activation was not significantly different between participants in all test conditions (Friedman's $\alpha$ llb $\beta 3$ activation: $p=0.062$; P-selectin expression: $p=0.293$ ). Normoxic exercise did not affect $\alpha$ llb $\beta 3$ activation (CRP: $p>0.99$; TRAP: $p=0.31$, compared to zero), or CRP-induced P-selectin expression ( $p=0.06$, compared to zero), but slightly decreased TRAP-induced $P$-selectin expression ( $p=0.03$, compared to zero). 

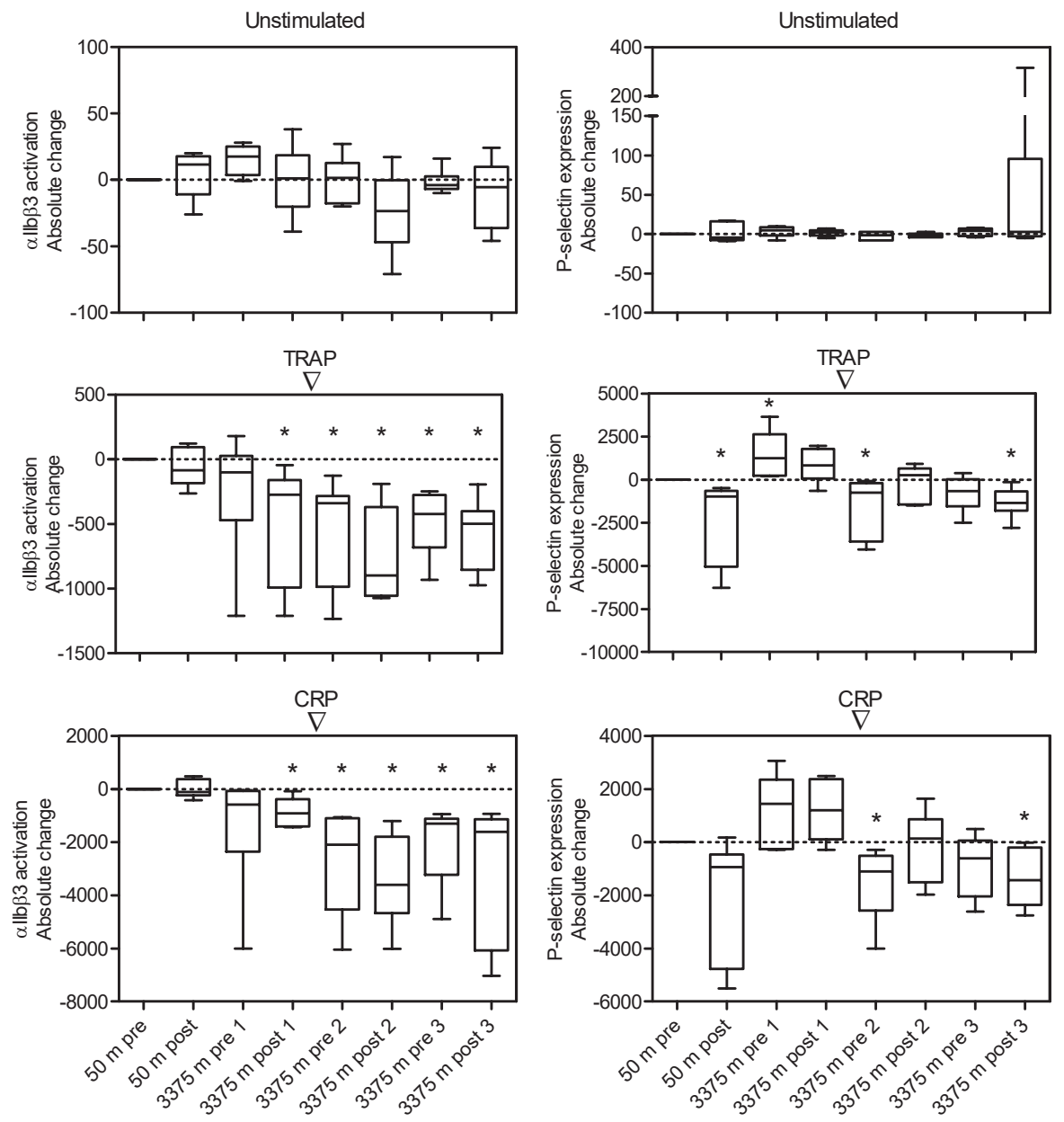

Figure 5. Influence of normoxic and hypoxic exercises on platelet activation. Platelet activation was measured before and after 2 hours of strenuous exercise, once at $50 \mathrm{~m}$ and three times at $3,375 \mathrm{~m}$ altitude. Platelets were stimulated with the agonist thrombin receptor-activating peptide (TRAP, $30 \mu \mathrm{mol} / \mathrm{L}$, middle panels) and collagen-related peptide (CRP, $5 \mu \mathrm{g} / \mathrm{L}$, bottom panels). An unstimulated condition was included as a control (top panels), showing no significant differences in baseline platelet activation between participants. Platelet activation was measured as $\alpha$ llb $\beta 3$ activation and P-selectin expression in median fluorescence intensity. Results are expressed as the absolute change of $50 \mathrm{~m}$ pre-exercise; a value above 0 indicates an increase, and a value below 0 indicates a decrease. $s=p<0.05$ (Friedman's test); * $p<0.05$ compared with zero (Wilcoxon signed-rank test).

CLT was not affected significantly by the normoxic exercise (see Table S1 section E). 


\section{Effect of ascent to high altitude on TG, platelet activation and clot lysis time}

The ascent to 3,375 $\mathrm{m}$ altitude did not cause significant changes in Peak and ETP of whole blood TG, see Figure 3. In PRP TG, the ETP was decreased at high altitude ( $p=0.03$, compared to zero), while the Peak was decreased as well but did not reach significance ( $p=0.06$, compared to zero). In PPP TG, at 5 PM TF the ETP was increased at high altitude ( $p=0.03$, compared to zero), with no change in Peak ( $p>0.99$, compared to zero). PPP TG at 1 pM TF (Peak and ETP both $p=0.84$, compared to zero) and CTI-1 pM TF (Peak: $p=0.09$ and ETP: $p=0.84$, compared to zero) did not change due to the increased altitude, see Figure 4.

Platelet $\alpha$ llb $\beta 3$ activation did not change directly after ascent to high altitude (CRP: p>0.99; TRAP: $p=0.22$, compared to zero), whereas TRAP-induced P-selectin expression initially increased $(p=0.03$, compared to zero), as shown in Figure 5 . Overall, the stay at high altitude depressed platelet $\alpha$ llb $\beta 3$ activation triggered by both CRP and TRAP (CRP: Friedman's $p<0.0001$; TRAP: Friedman's $p<0.001$ ). The CRP-induced $\alpha$ llb $\beta 3$ activation was maximally decreased at day 2 post-exercise ( $p=0.03$, compared to zero).

CLT was not affected significantly by altitude (see Table S1 section E).

\section{Effect of repeated hypoxic exercise on TG, platelet activation and clot lysis time}

Overall, in WB at 0 pM TF, TG Peak (Friedman's $p=0.003$ ) as well as ETP (Friedman's $p=0.02$ ) remained increased during the three days at high altitude, see Figure 3 . There was no evident effect of repetitive hypoxic exercise on WB TG. Peak and ETP in PRP remained consistently decreased at high altitude (Friedman's $p<0.001$, and $p<0.0001$, respectively). Hypoxic exercise appeared to further decrease PRP TC during the three test days at high altitude.

As shown in Figure 4, TG in PPP was not affected consistently by hypoxic exercise. Peak TG and ETP at 1 pM TF were overall not significantly different (Friedman's $p=0.19$, and $p=0.30$ respectively). However, there were some differences compared to $50 \mathrm{~m}$ preexercise. The Peak at 5 pM TF was slightly increased after hypoxic exercise on day 2 and 3 (both $p=0.03$, compared to zero). Moreover, ETP at 5 pM TF remained slightly increased at high altitude (Friedman's $p=0.003$ ), which was most prominent at day 3 (both pre- and post-exercise $p=0.03$, compared to zero). Peak TG in CTI-anticoagulated PPP was slightly increased during day 2 and 3 at high altitude (Friedman's $p=0.01$ ) and increased more post-exercise on day 2 ( $p=0.03$, compared to zero). 
There was no consistent exercise-dependent effect on platelet $\alpha$ llb $\beta 3$ activation at high altitude (see Figure 5). The depressed TRAP-induced P-selectin expression that was seen after normoxic exercise, was not seen after hypoxic exercise on day 1 ( $p=0.16$, compared to zero) and day 2 ( $p>0.99$, compared to zero), but was observed on day 3 $(p=0.03$, compared to zero). Likewise, post-exercise P-selectin expression induced by CRP was unchanged at high altitude on the first two days ( $p=0.09$ on day $1, p>0.99$ on day 2 ), but was significantly decreased on day 3 ( $p=0.03$, compared to zero). Overall, CLT did not change during the three-day stay at high altitude (see Table S1 section E; Friedman's $\mathrm{p}=0.613$ ).

\section{DISCUSSION}

In this pilot study, we examined the effects of normoxic and hypoxic strenuous exercise during 2 hours on thrombin generation (TG), platelet activation and fibrinolysis in six active and healthy men. The hypoxic exercise tests were carried out on three consecutive days to determine whether haemostasis is modulated after repeated exercise in a hypoxic environment.

The participants were mildly hypoxic at 3,375 m altitude and acclimatized to the hypoxic environment at high altitude, by raising their haemoglobin $(\mathrm{Hb})$ levels and mean corpuscular haemoglobin concentration after a 3-day stay at 2,473 m plus 1 night at $3,375 \mathrm{~m}$ altitude. $\mathrm{Hb}$ production increases in a hypoxic environment through elevation of erythropoietin, a response that generally takes around 4 days to become apparent. ${ }^{32}$ Both normoxic and hypoxic exercise increased the granulocyte count, which returned to baseline every day. Acute exercise is known to induce transient neutrophilia. ${ }^{33}$ Neutrophils are the most abundant granulocytes and play an important role in destroying pathogenic invaders or cellular debris. Platelet count rose after the exercise tests, albeit non-significantly. Acute exercise is known to increase the number of platelets, which is thought to occur via mobilization of a splenic platelet pool through elevated catecholamines and/or shear stress. ${ }^{12,34}$ We found no signs of dehydration after the exercise tests or due to the altitude, as haematocrit $(\mathrm{Ht})$ and albumin did not increase. ${ }^{35}$

Normoxic exercise increased levels of VWF and active conformation VWF. The VWF level is known to be increased following exercise through release from endothelial WeibelPalade bodies. ${ }^{12}$ As a result of strenuous exercise, intravascular shear stress is increased and VWF is unfolded, exposing the A1 domain; this conformation is known as active VWF. ${ }^{36}$ This active VWF binds to platelets more readily and is hence more thrombogenic. ${ }^{37}$ Additionally, we found that normoxic exercise raised factor VIII (FVIII) levels, an 
effect that is also well known. FVIII circulates complexed with VWF, and therefore FVIII is most likely secondarily increased by VWF. ${ }^{12}$ Moreover, normoxic exercise increased the Peak TG, but not endogenous thrombin potential (ETP) in both platelet-poor and platelet-rich plasma. Increased Peak TG may be due to clotting activation mediated by contact factors, of which FVIII is one. ${ }^{38}$

Recently, we performed another small-scale cycling study, in which 5 participants cycled $80 \mathrm{~km}$ at sea level on 3 consecutive days. ${ }^{39}$ The normoxic cycling study also showed that exercise significantly increases VWF (antigen, propeptide and active conformation) levels, FVIII levels, and Peak TG. No increase in ETP was observed after cycling, which is in agreement with another large strenuous cycling study. ${ }^{10,39}$

In the current study, the normoxic exercise slightly decreased TRAP-induced P-selectin expression and did not have an effect on $\alpha$ llb $\beta 3$ activation in our active volunteers. In contrast with these findings, there is an abundancy of data showing that physical exercise induces platelet activation. ${ }^{12}$ However, there appears to be a different platelet response to exercise in physically trained subjects compared to sedentary subjects, as it has been found before that strenuous exercise does not induce platelet hyperreactivity in trained individuals. ${ }^{40}$ Our subjects were trained, and it is possible that their platelets therefore did not become hyperreactive. Moreover, in the other small-scale cycling study, platelet $\mathrm{P}$-selectin expression showed a similar decrease after exercise. ${ }^{39}$

There was no effect of normoxic exercise on fibrinolysis in this study, as clot lysis time (CLT) did not change significantly. Short-term strenuous exercise is known to augment fibrinolysis through increased tissue-type plasminogen activator (t-PA) levels, which is released from endothelial cells, and decreased plasminogen activator inhibitor (PAI)-1 levels, the primary inhibitor of t-PA that is released from activated platelets. ${ }^{12}$ The CLT is a global measurement for fibrinolysis, and may not be sensitive enough to pick up the exercise-induced hyperfibrinolysis.

We found that ascent to 3,375 m altitude mildly increased whole blood TG and FVIII levels, while it did not initially change platelet activation markers. However, at day two and three at high altitude, TG in PPP was slightly increased, and platelet $\alpha$ llb $\beta 3$ activation and platelet-dependent TG were depressed. Hypoxia was previously found to decrease ex vivo platelet $\alpha$ llb $\beta 3$ activation and aggregation. ${ }^{18}$ In another recent high altitude study performed by our group, we found that ascent to 3,883 m altitude increased TC but decreased platelet activation in healthy inactive volunteers. ${ }^{13}$ However, in that study, the hypoxia was more profound and was accompanied by slightly increased lactate levels. Therefore, these two studies should not be compared one on one. 
In this study, there was no effect of the high-altitude sojourn on fibrinolysis. Data on the effect of systemic hypoxia on fibrinolysis are scarce. In one other study, it was found that hypobaric hypoxia equivalent to $2,438 \mathrm{~m}$ altitude does not change t-PA and PAI-1 levels. ${ }^{41}$

We found that the hypoxic exercise tests elevated VWF, active VWF and FVIII as well. Over the course of the three days at high altitude, the amplitude of exercise-induced elevation of VWF and active VWF decreased slightly, suggesting that either the VWF supply or the endothelial response to physical stress was diminishing. In contrast to the exercise test at sea level, the hypoxic exercise tests did not have a consistent effect on Peak TG. Platelet-dependent TG even appeared to show an exercise-related decrease. The diminished platelet $\alpha$ llb $\beta 3$ activation at high altitude was not further aggravated by hypoxic exercise.

The findings from this hypoxic cycling study are in part similar to the findings of the other small-scale normoxic cycling study. In that study, the FVIII increase was tapered over three days, an effect that was also apparent by a decreasing amplitude of Peak TG in plasma. Moreover, platelet $\mathrm{P}$-selectin expression decreased after repeated exercise and did not recover fully, indicating exhaustion of the platelet response to repetitive exercise. ${ }^{39}$ However, there is one major difference. In the current study, staying in a hypoxic environment appeared to further depress platelet $\alpha$ llb $\beta 3$ activation and plateletdependent TG.

We did not find an effect of repeated hypoxic exercise on fibrinolysis in the current pilot study. In the previous small-scale normoxic cycling study, clot lysis time decreased very slightly on the first two days after exercise and decreased significantly on the third day. In another experimental study, it was found that severe hypoxia (equivalent to an altitude of $4,600 \mathrm{~m}$ ) enhanced fibrinolytic activity after exercise, by decreasing the exercise-induced increase in PAI- $1 .{ }^{17}$

This study has some limitations. The small number of participants precluded the use of parametric statistics. Nonparametric statistical analyses such as the Friedman test are quite conservative and are therefore relatively insensitive to clinically relevant differences. ${ }^{42}$ For instance, the heart rate before and after exercise was not identified as statistically different, although they were clinically clearly different (see Figure S1 and Table S1 section A). Therefore, we also used descriptive statistics to discover trends in the data, although this is more prone to biased interpretation. 
While the percentage of maximum $\mathrm{O}_{2}$ consumption ( $\left.\% \mathrm{VO}_{2} \max \right)$ is considered the gold standard for standardization of exercise intensity, we used the percentage of HRR (\%HRR). The relationship between \%HRR and \% $\mathrm{VO}_{2}$ max is not linear, especially at low exercise intensity and in subjects with low cardiorespiratory fitness, which limits its use in exercise physiology research. ${ }^{23}$ However, the use of $\mathrm{VO}_{2}$ max in an environment with decreased athmospheric $\mathrm{O}_{2}$ pressure is of limited value. Acute hypoxia reduces the $\mathrm{VO}_{2} \max$, and despite increases in haemoglobin and $\mathrm{O}_{2}$ saturation that can normalize arterial $\mathrm{O}_{2}$ content after acclimatization, $\mathrm{VO}_{2}$ max remains lower at high altitude. ${ }^{17,} 32$ Our goal was to standardize strenuous exercise both at sea level and high altitude. The $\% \mathrm{HRR}$ is not affected by athmospheric $\mathrm{O}_{2}$ pressure, and is adequate for standardization of strenuous exercise. ${ }^{23}$

Furthermore, we did not include any women in this study to minimize heterogeneity in our small group of participants. However, women are known to have a different haemostatic response to exercise, and therefore these results should not be extrapolated to them. ${ }^{10,43}$ Moreover, there was a considerable age difference between the participants (18-48 years). Exercise tolerance and endothelial function are known to be affected by age ${ }^{44}$, hence this (in combination with the small sample size) has contributed to the relatively wide distributions of the data. All subjects were physically fit, exercising between 4 and 7.5 hours per week. Of note, 4 out of 6 subjects were regular cyclists. These variations could also potentially influence the response to our exercise protocol.

Taken together, the current data should be interpreted with caution. A large follow-up study is necessary for identifying differences that are both clinically and statistically relevant, and to investigate if the effect is present in women as well.

\section{CONCLUSION}

Strenuous exercise induces a procoagulant phenotype that is mediated by the endothelium, by increasing VWF and secondarily raising FVIII levels. The amplitude of the endothelial response to exercise decreases after repetitive exercise. A hypoxic environment may further increase the exhaustive effect of repetitive exercise on haemostasis by depressing platelet aggregation potential and platelet-dependent TG. These results warrant further investigations, to determine how much time is required for the endothelial response to exercise to recover and whether platelet activation is depressed more during prolonged exposure to a hypoxic environment. 


\section{ACINOWLEDGEMENTS}

We are greatly indebted to Ms. M.R. van den Brink, MD for facilitating the ergometry tests that were performed as a part of the work-up for this study. Ms. V.J.F. Strijbis is thanked for her indispensable technical support. Furthermore, we are grateful to all six volunteers, who made time for participating in this pilot study and performed at their best during the exercise tests, especially at high altitude. 


\section{REFERENCES}

1. Albano AJ, Thompson PD, Kapur NK. Acute coronary thrombosis in Boston marathon runners. N Engl J Med. 2012;366(2):184-5.

2. Kim JH, Malhotra R, Chiampas G, d'Hemecourt P, Troyanos C, Cianca J, et al. Cardiac arrest during long-distance running races. N Engl J Med. 2012;366(2):130-40.

3. Tak S, Tak S. Lower extremity deep vein thrombosis after heavy exertion. BMJ Case Rep. 2013;2013.

4. Anand AC, Jha SK, Saha A, Sharma V, Adya CM. Thrombosis as a complication of extended stay at high altitude. Natl Med J India. 2001;14(4):197-201.

5. Anand AC, Saha A, Seth AK, Chopra GS, Nair V, Sharma V. Symptomatic portal system thrombosis in soldiers due to extended stay at extreme altitude. J Gastroenterol Hepatol. 2005;20(5):777-83.

6. Damodar D, Donnally CJ, 3rd, Sheu JI, Law TY, Roche MW, Hernandez VH. A Higher Altitude Is an Independent Risk Factor for Venous Thromboembolisms After Total Hip Arthroplasty. J Arthroplasty. 2018;33(8):2627-30.

7. Tyson JJ, Bjerke BP, Genuario JW, Noonan TJ. Thromboembolic Events After Arthroscopic Knee Surgery: Increased Risk at High Elevation. Arthroscopy. 2016;32(11):2350-4.

8. Indermuehle A, Cook S, Marty $\mathrm{H}$. A young mountaineer surviving sudden cardiac arrest at high altitude. BMJ Case Rep. 2010;2010.

9. Hilberg T, Prasa D, Sturzebecher J, Glaser D, Schneider K, Gabriel HH. Blood coagulation and fibrinolysis after extreme short-term exercise. Thromb Res. 2003;109(5-6):271-7.

10. Huskens D, Roest M, Remijn JA, Konings J, Kremers RM, Bloemen S, et al. Strenuous exercise induces a hyperreactive rebalanced haemostatic state that is more pronounced in men. Thromb Haemost. 2016;115(6):1109-19.

11. Sedgwick MJ, Thompson M, Garnham J, Thackray AE, Barrett LA, Powis M, et al. Acute highintensity interval rowing increases thrombin generation in healthy men. Eur J Appl Physiol. 2016;116(6):1139-48.

12. Kicken $\mathrm{CH}$, Miszta $\mathrm{A}$, Kelchtermans $\mathrm{H}$, De Laat $\mathrm{B}$. Hemostasis during Extreme Exertion. Semin Thromb Hemost. 2018;44(7):640-50.

13. Kicken CH, Ninivaggi M, Konings J, Moorlag M, Huskens D, Remijn JA, et al. Hypobaric Hypoxia Causes Elevated Thrombin Generation Mediated by FVIII that is Balanced by Decreased Platelet Activation. Thromb Haemost. 2018;118(5):883-92.

14. Wang JS, Cheng ML, Yen HC, Lou BS, Liu HC. Vitamin E suppresses enhancement of factor VIII-dependent thrombin generation by systemic hypoxia. Stroke. 2009;40(2):656-9.

15. Fall L, New KJ, Evans KA, Bailey DM. Arterial hypoxaemia and its impact on coagulation: significance of altered redox homeostasis. J Clin Pathol. 2015;68(9):752-4.

16. Chen YC, Ho CW, Tsai HH, Wang JS. Interval and continuous exercise regimens suppress neutrophil-derived microparticle formation and neutrophil-promoted thrombin generation under hypoxic stress. Clin Sci (Lond). 2015;128(7):425-36.

17. DeLoughery TG, Robertson DG, Smith CA, Sauer D. Moderate hypoxia suppresses exerciseinduced procoagulant changes. Br J Haematol. 2004;125(3):369-72.

18. Kiouptsi K, Gambaryan S, Walter E, Walter U, Jurk K, Reinhardt C. Hypoxia impairs agonist-induced integrin alphallbbeta3 activation and platelet aggregation. Sci Rep. 2017;7(1):7621. 
19. Wang JS, Chang YL, Chen YC, Tsai HH, Fu TC. Effects of normoxic and hypoxic exercise regimens on monocyte-mediated thrombin generation in sedentary men. Clin Sci (Lond). 2015;129(4):363-74.

20. Ninivaggi $M$, de Laat $M$, Lance $M$ M, Kicken $C H$, Pelkmans $L$, Bloemen $S$, et al. Hypoxia Induces a Prothrombotic State Independently of the Physical Activity. PLoS One. 2015;10(10):e0141797.

21. HyLown. Power and Sample Size Calculator: Compare k Means (1-Way ANOVA Pairwise, 2-Sided Equality). Available at: http://hylown.com/. Accessed January 3, 2017.

22. Gibbons RJ, Balady GJ, Bricker JT, Chaitman BR, Fletcher GF, Froelicher VF, et al. ACC/AHA 2002 guideline update for exercise testing: summary article: a report of the American College of Cardiology/American Heart Association Task Force on Practice Guidelines (Committee to Update the 1997 Exercise Testing Guidelines). Circulation. 2002;106(14):1883-92.

23. da Cunha FA, Farinatti Pde T, Midgley AW. Methodological and practical application issues in exercise prescription using the heart rate reserve and oxygen uptake reserve methods. J Sci Med Sport. 2011;14(1):46-57.

24. Roach R, Bärtsch P, Hackett P, Oelz O. Lake Louise AMS Scoring Consensus Committee. The Lake Louise consensus on the quantification of altitude illness. In: Hypoxia and Molecular Medicine. Burlington, VT: Queen City Press; 1993.

25. van der Vorm LN, Li L, Huskens D, Chayoua W, Kelchtermans H, de Groot PG, et al. Analytical characterization and reference interval of an enzyme-linked immunosorbent assay for active von Willebrand factor. PLoS One. 2019;14(2):e0211961.

26. Ninivaggi M, Apitz-Castro R, Dargaud Y, de Laat B, Hemker HC, Lindhout T. Whole-blood thrombin generation monitored with a calibrated automated thrombogram-based assay. Clin Chem. 2012;58(8):1252-9.

27. Hemker HC, Kremers R. Data management in thrombin generation. Thromb Res. 2013;131(1):3-11.

28. Hemker HC, Giesen P, Al Dieri R, Regnault V, de Smedt E, Wagenvoord R, et al. Calibrated automated thrombin generation measurement in clotting plasma. Pathophysiol Haemost Thromb. 2003;33(1):4-15.

29. Spronk HM, Dielis AW, De Smedt E, van Oerle R, Fens D, Prins MH, et al. Assessment of thrombin generation II: Validation of the Calibrated Automated Thrombogram in platelet-poor plasma in a clinical laboratory. Thromb Haemost. 2008;100(2):362-4.

30. Huskens D, Sang Y, Konings J, van der Vorm L, de Laat B, Kelchtermans H, et al. Standardization and reference ranges for whole blood platelet function measurements using a flow cytometric platelet activation test. PLoS One. 2018;13(2):e0192079.

31. Vickers AJ. The use of percentage change from baseline as an outcome in a controlled trial is statistically inefficient: a simulation study. BMC Med Res Methodol. 2001;1:6.

32. Calbet JA, Boushel R, Radegran G, Sondergaard H, Wagner PD, Saltin B. Why is VO2 max after altitude acclimatization still reduced despite normalization of arterial $\mathrm{O} 2$ content? Am J Physiol Regul Integr Comp Physiol. 2003;284(2):R304-16.

33. Gabriel H, Kindermann W. The acute immune response to exercise: what does it mean? Int ] Sports Med. 1997;18 Suppl 1:S28-45.

34. Chamberlain KG, Tong M, Penington DG. Properties of the exchangeable splenic platelets released into the circulation during exercise-induced thrombocytosis. Am J Hematol. 1990;34(3):161-8. 
35. Dill DB, Costill DL. Calculation of percentage changes in volumes of blood, plasma, and red cells in dehydration. J Appl Physiol. 1974;37(2):247-8.

36. Huizinga EG, Tsuji S, Romijn RA, Schiphorst ME, de Groot PG, Sixma JJ, et al. Structures of glycoprotein Ibalpha and its complex with von Willebrand factor A1 domain. Science. 2002;297(5584):1176-9.

37. Ruggeri ZM. The role of von Willebrand factor in thrombus formation. Thromb Res. 2007;120 Suppl 1:S5-9.

38. Bloemen S, Huskens D, Konings J, Kremers R, Miszta A, de Laat B, et al. Interindividual variability and normal ranges of whole blood and plasma thrombin generation. J Appl Lab Med 2017:;2(2):150-64.

39. van der Vorm LN, Huskens D, Kicken CH, Remijn JA, Roest M, de Laat B, et al. Effects of Repeated Bouts of Exercise on the Hemostatic System. Semin Thromb Hemost. 2018;44(8):710-22.

40. Kestin AS, Ellis PA, Barnard MR, Errichetti A, Rosner BA, Michelson AD. Effect of strenuous exercise on platelet activation state and reactivity. Circulation. 1993;88(4 Pt 1):1502-11.

41. Toff WD, Jones Cl, Ford I, Pearse RJ, Watson HG, Watt SJ, et al. Effect of hypobaric hypoxia, simulating conditions during long-haul air travel, on coagulation, fibrinolysis, platelet function, and endothelial activation. JAMA. 2006;295(19):2251-61.

42. Dwivedi AK, Mallawaarachchi I, Alvarado LA. Analysis of small sample size studies using nonparametric bootstrap test with pooled resampling method. Stat Med. 2017;36(14):2187205.

43. van Loon JE, Sonneveld MA, Praet SF, de Maat MP, Leebeek FW. Performance related factors are the main determinants of the von Willebrand factor response to exhaustive physical exercise. PLoS One. 2014;9(3):e91687.

44. Seals DR, Walker AE, Pierce GL, Lesniewski LA. Habitual exercise and vascular ageing. J Physiol. 2009;587(Pt 23):5541-9. 


\section{SUPPLEMENTAL DATA}

\section{Supplemental Figures}

Subject 1

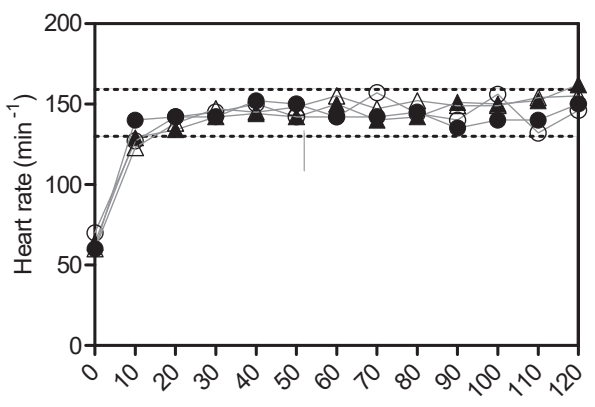

Subject 3

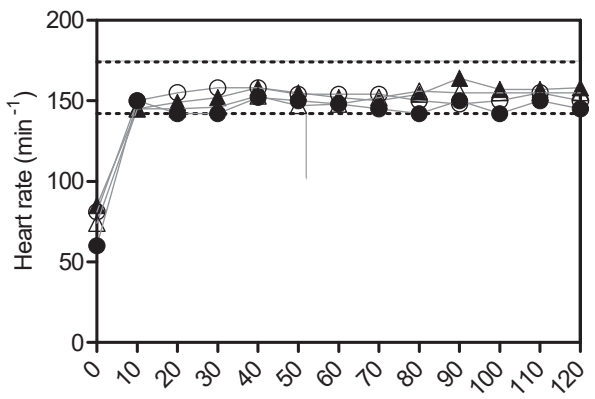

Subject 5

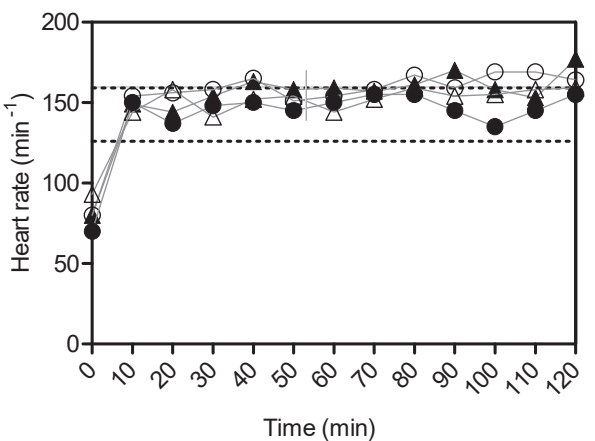

Subject 2

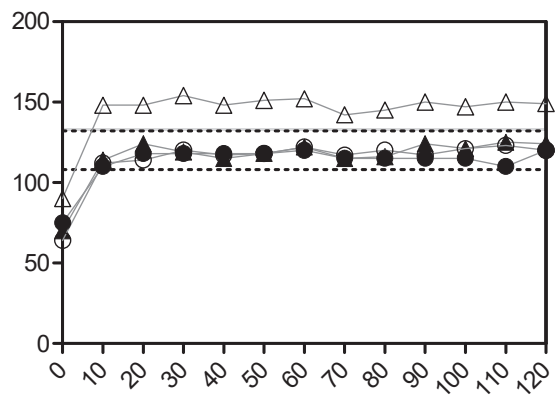

Subject 4

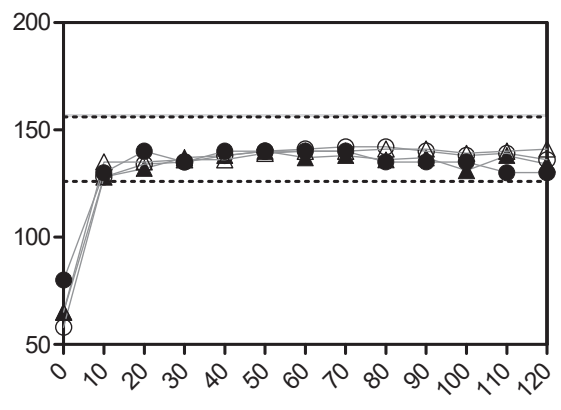

Subject 6

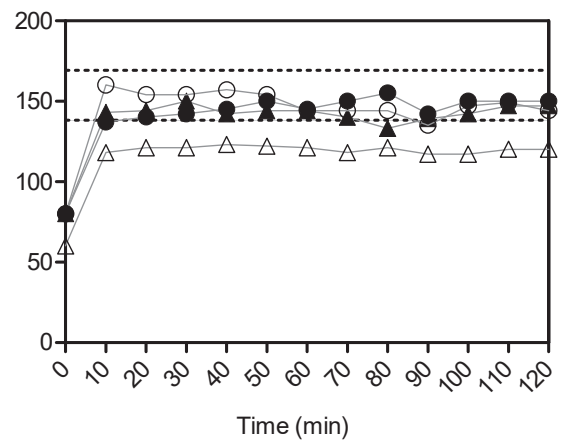

Figure S1. Individual heart rate traces during the exercise tests. Participants performed monitored exercise for 2 hours on 4 occasions: 1 time at $50 \mathrm{~m}$ altitude ( ), and 3 times at 3,375 m altitude ( $r$ day 1, i day $2, p$ day 3 ). Heart rate (HR, beats per minute) was noted every 10 minutes and participants were encouraged to keep their HR between $60-85 \%$ of their personal predefined heart rate reserve (HRR). The grey area on the graphs represents $60-85 \%$ of HRR. 


\section{Supplemental Tables}

Table S1. Vital signs, biochemical markers, blood count, coagulation factor levels and clot lysis time.

\begin{tabular}{|c|c|c|c|c|c|c|c|c|c|}
\hline & \multicolumn{2}{|c|}{$50 \mathrm{~m}$ altitude } & \multicolumn{6}{|c|}{$3,375 \mathrm{~m}$ altitude } & \multirow[t]{2}{*}{$\mathrm{p}$-value } \\
\hline & Pre & Post & Pre 1 & Post 1 & Pre 2 & Post 2 & Pre 3 & Post 3 & \\
\hline \multicolumn{10}{|c|}{ A. Vital signs } \\
\hline $\mathrm{SpO}_{2}$ & $99[1]$ & $98[2]$ & $93[3]$ & $90 *[4]$ & $92[3]$ & 91*[7] & $92 \div[2]$ & $92[3]$ & 0.0004 \\
\hline HR & $56[13]$ & $148[24]$ & $64[17]$ & $152 *[20]$ & $59[13]$ & $145[21]$ & $69[13]$ & $153 *[34]$ & $<0.0001$ \\
\hline \multicolumn{10}{|c|}{ B. Biochemical markers } \\
\hline Lactate & $1.63[0.33]$ & 2.17 [1.04] & $2.16[0.84]$ & $2.1[0.24]$ & $1.41[0.24]$ & $1.98[0.62]$ & $1.67[0.66]$ & $1.91[0.62]$ & 0.0035 \\
\hline Creatinine & $68.7[13.3]$ & $74.6 *[13.8]$ & $69.8[19.6]$ & $72.5[14.5]$ & $68.0[16.1]$ & $70.9[11.1]$ & $69[13.4]$ & $73.4[8.23]$ & 0.0074 \\
\hline Urea & $5.3[1.38]$ & $5.9[1.35]$ & $4.95[1.15]$ & $4.65[0.75]$ & $4.75[1.33]$ & $4.95[1.27]$ & $5.0[0.78]$ & $4.65[1.1]$ & 0.0124 \\
\hline Albumin & $35.4[2.8]$ & $38.1[5.3]$ & $36.4[4.7]$ & $38.1[4.3]$ & $35.9[3.0]$ & $36.4[4.7]$ & $33.45[1.1]$ & $35.85[4.4]$ & 0.0073 \\
\hline CRP & $0.45[0.78]$ & $0.55[0.82]$ & $0.45[2.0]$ & $0.4[1.88]$ & $0.7[1.1]$ & $0.6[1.25]$ & $0.7[1.43]$ & $0.75[1.4]$ & 0.0155 \\
\hline \multicolumn{10}{|c|}{ C. Blood count } \\
\hline RBC & $4.7[0.7]$ & $4.8[0.8]$ & $5.2[0.3]$ & $5.1[0.6]$ & $5.4 *[0.3]$ & $5.3[0.2]$ & $4.9[0.5]$ & $5.1[0.6]$ & 0.0026 \\
\hline $\mathrm{Ht}$ & $0.42[0.04]$ & $0.42[0.05]$ & $0.45[0.02]$ & 0.44 [0.02] & $0.46 *[0.03]$ & $0.45[0.03]$ & $0.44[0.04]$ & $0.44[0.02]$ & 0.0117 \\
\hline $\mathrm{Hb}$ & $8.2[0.4]$ & $8.5[1.4]$ & $10.0[0.6]$ & $10.1 *[1.0]$ & $10.2 *[0.8]$ & $10.0 *[1.0]$ & $9.6[0.8]$ & $9.8[0.7]$ & $<0.0001$ \\
\hline $\mathrm{MCHC}$ & $19.9[0.9]$ & $20.1[0.6]$ & $21.9[1.1]$ & $22.8 *[1.4]$ & $22.5 *[0.9]$ & $22.4[1.0]$ & $22.1[0.6]$ & $22.41 .3]$ & $<0.0001$ \\
\hline WBC & $5.6[2.4]$ & $7.2[1.3]$ & $6.5[2.0]$ & $8.0 *[3.6]$ & $7.3[1.8]$ & $8.6 *[4.0]$ & $6.7[1.7]$ & $7.9[2.5]$ & 0.0016 \\
\hline Gr & $3.4[2.1]$ & $4.8[1.3]$ & $4.0[1.3]$ & $5.3 \div[2.7]$ & $4.8[2.0]$ & $5.4 \div[2.7]$ & $4.4[1.5]$ & $5.4 *[2.1]$ & 0.0002 \\
\hline LY & $2.2[0.9]$ & $2.1[1.2]$ & $2.4[0.9]$ & $2.1[0.9]$ & $2.1[0.4]$ & $2.1[1.5]$ & $2.1[0.6]$ & $2.3[1.1]$ & 0.7734 \\
\hline MO & $0.3[0.1]$ & $0.3[0.2]$ & $0.4[0.2]$ & $0.4[0.1]$ & $0.4[0.2]$ & $0.4[0.2]$ & $0.3[0.3]$ & $0.3[0.2]$ & 0.6597 \\
\hline PC & 238 [60] & 267 [85] & 251 [60] & 268 [84] & 229 [57] & 281 [63] & 234 [41] & 264 [59] & 0.0629 \\
\hline \multicolumn{10}{|c|}{ D. Coagulation factor levels } \\
\hline VWF:Ag & $93[61]$ & $152 *[86]$ & $89[68]$ & $142[78]$ & $99[88]$ & $125[83]$ & $93[76]$ & $121[87]$ & 0.0002 \\
\hline aVWF & $124[32]$ & $173 *[117]$ & $131[43]$ & $197 *[61]$ & $148[68]$ & $177 *[87]$ & $127[54]$ & 148 [53] & 0.0006 \\
\hline FVIII:C & $108[18]$ & $161 *[97]$ & $114[33]$ & $163[112]$ & $116[39]$ & $164[40]$ & $116[41]$ & $154[48]$ & 0.0009 \\
\hline $\mathrm{Fg}$ & $2.6[1.1]$ & $2.6[1.6]$ & $2.8[1.2]$ & $2.7[1.1]$ & $2.9[1.2]$ & $2.7[1.3]$ & $2.7[1.1]$ & $2.8[1.2]$ & 0.0923 \\
\hline AT & $95[15]$ & $99[13]$ & $98[10]$ & $97[14]$ & $96[9]$ & $101[16]$ & 93 [9] & $95[6]$ & 0.1536 \\
\hline
\end{tabular}

\section{E. Fibrinolysis}

CLT 24.0[16.8] 26.0[18.4] 26.7[19.2] 26.3[15.2] 25.8 [20.0] 26.0[19.0] 23.5[15.4] 23.5[16.6] 0.6131

(Caption Table S1).. All parameters were measured at $50 \mathrm{~m}$ and 3,375 m altitude, before and after exercise $(n=6)$, and are expressed as median [interquartile range]. P-value $=$ Friedman's test, $\mathrm{p}<0.05$ was considered statistically significant. Dunn's post-hoc analysis: * $=p<0.05$ compared to $50 \mathrm{~m}$ pre-exercise. A. Vital signs: peripheral oxygen saturation $\left(\mathrm{SpO}_{2}, \%\right)$, heart rate (HR, beats/ $\mathrm{min})$. B. Biochemical markers: lactate $(\mathrm{mmol} / \mathrm{L})$, creatinine $(\mu \mathrm{mol} / \mathrm{L})$, urea $(\mathrm{mmol} / \mathrm{L})$, albumin $(\mathrm{g} / \mathrm{L})$ and C-reactive protein (CRP, $\mathrm{mg} / \mathrm{L})$. C. Blood count: red blood cell count $\left(\mathrm{RBC}, \cdot 10^{12} / \mathrm{L}\right)$, haematocrit $(\mathrm{Ht}, \mathrm{L} / \mathrm{L})$, (haemoglobin $(\mathrm{Hb}, \mathrm{mmol} / \mathrm{L})$, mean corpuscular haemoglobin concentration (MCHC, $\mathrm{mmol} / \mathrm{L})$ white blood cell count $\left(\mathrm{WBC}, 10^{9} / \mathrm{L}\right)$, granulocyte count $\left(\mathrm{Gr}, 10^{9} / \mathrm{L}\right)$, lymphocyte count (LY, $\left.10^{9} / \mathrm{L}\right)$, monocyte count $\left(\mathrm{MO}, \cdot 10^{9} / \mathrm{L}\right)$, platelet count $\left(\mathrm{PC}, \cdot 10^{9} / \mathrm{L}\right)$. D. Coagulation factor levels: von Willebrand Factor antigen (VWF:Ag, \%), VWF in active conformation (aVWF, \% of normal pool plasma), factor VIII concentration (FVIII:C, \%), fibrinogen levels (Fg, g/L), antithrombin (AT, \%). E. Fibrinolysis: clot lysis time (CLT, min), defined as the time from half-maximum clot formation to half-maximum clot degradation. 
Table S2. Thrombin generation.

\begin{tabular}{|c|c|c|c|c|c|c|c|c|c|}
\hline & \multicolumn{8}{|c|}{$50 \mathrm{~m}$ altitude $\quad 3,375 \mathrm{~m}$ altitude } & \multirow[t]{2}{*}{ p-value } \\
\hline & Pre & Post & Pre 1 & Post 1 & Pre 2 & Post 2 & Pre 3 & Post 3 & \\
\hline \multicolumn{10}{|l|}{ A. WB O pM TF } \\
\hline Peak (nM) & 0 & $\begin{array}{c}+4.5 \\
{[-3.2 ;+21]}\end{array}$ & $\begin{array}{c}+27 \\
{[+9.0 ;+42]}\end{array}$ & $\begin{array}{c}+41 \\
{[+30 ;+50]}\end{array}$ & $\begin{array}{c}+31 \\
{[+24 ; 39]}\end{array}$ & $\begin{array}{c}+54 \\
{[+21 ;+59]}\end{array}$ & $\begin{array}{c}+16 \\
{[+10 ;+43]}\end{array}$ & $\begin{array}{c}+28 \\
{[+14 ;+52]}\end{array}$ & 0.0028 \\
\hline $\operatorname{ETP}(\mathrm{nM} \cdot \mathrm{min})$ & 0 & $\begin{array}{c}+35 \\
{[-40 ;+38]}\end{array}$ & $\begin{array}{c}+157 \\
{[+42 ;+177]}\end{array}$ & $\begin{array}{c}+130 \\
{[+116 ;+167]}\end{array}$ & $\begin{array}{c}+39 \\
{[+7.1 ;+93]}\end{array}$ & $\begin{array}{c}+122 \\
{[+7.4 ;+173]}\end{array}$ & $\begin{array}{c}+5.9 \\
{[-11 ;+166]}\end{array}$ & $\begin{array}{c}+3.3 \\
{[-9.6 ;+73]}\end{array}$ & 0.0202 \\
\hline \multicolumn{10}{|c|}{ B. WB 0.5 pM TF } \\
\hline Peak (nM) & 0 & $\begin{array}{c}-4.0 \\
{[-13 ;+9.8]}\end{array}$ & $\begin{array}{c}+3.4 \\
{[-17 ;+7.4]}\end{array}$ & $\begin{array}{c}-8.2 \\
{[-24 ;+17]}\end{array}$ & $\begin{array}{c}+5.5 \\
{[-9.7 ;+15]}\end{array}$ & $\begin{array}{c}-1.33 \\
{[-8.9 ;+32]}\end{array}$ & $\begin{array}{c}+14 \\
{[-5.3 ;+39]}\end{array}$ & $\begin{array}{c}+0.8 \\
{[-2.6 ;+9.8]}\end{array}$ & 0.6762 \\
\hline $\operatorname{ETP}(\mathrm{nM} \cdot \mathrm{min})$ & 0 & $\begin{array}{c}-11 \\
{[-67 ;+39]}\end{array}$ & $\begin{array}{c}+112 \\
{[-1.8 ;+151]}\end{array}$ & $\begin{array}{c}+81 \\
{[+45 ;+170]}\end{array}$ & $\begin{array}{c}+53 \\
{[-15 ;+84]}\end{array}$ & $\begin{array}{c}+84 \\
{[+20 ;+181]}\end{array}$ & $\begin{array}{c}+55 \\
{[-2.9 ;+195]}\end{array}$ & $\begin{array}{c}+11 \\
{[-6.6 ;+39]}\end{array}$ & 0.0387 \\
\hline \multicolumn{10}{|l|}{ C. PRP } \\
\hline Peak (\%) & 0 & $\begin{array}{c}+23 * \\
{[+15 ;+49]}\end{array}$ & $\begin{array}{c}-43 \\
{[-59 ;-29]}\end{array}$ & $\begin{array}{c}-58 * \\
{[-96 ;-13]}\end{array}$ & $\begin{array}{c}-13.85 \\
{[-51 ;-2.8]}\end{array}$ & $\begin{array}{c}-26 \\
{[-41 ;+14]}\end{array}$ & $\begin{array}{c}-30 \\
{[-42 ;+9.1]}\end{array}$ & $\begin{array}{c}-39 \\
{[-73 ;-14]}\end{array}$ & 0.0003 \\
\hline ETP (\%) & 0 & $\begin{array}{c}+4.1 \\
{[-0,8 ;+19]}\end{array}$ & $\begin{array}{c}-41 * \\
{[-62 ;-28]}\end{array}$ & $\begin{array}{c}-58 * \\
{[-73 ;-26]}\end{array}$ & $\begin{array}{c}-28 \\
{[-54 ;-15]}\end{array}$ & $\begin{array}{c}-41 * \\
{[-52 ;-20]}\end{array}$ & $\begin{array}{c}-30 * \\
{[-41 ;-7.5]}\end{array}$ & $\begin{array}{c}-50 * \\
{[-62 ;-21]}\end{array}$ & $<0.0001$ \\
\hline \multicolumn{10}{|l|}{ D. PPP 1 pM TF } \\
\hline Peak (\%) & 0 & $\begin{array}{c}+20 \\
{[+3.7 ;+50]}\end{array}$ & $\begin{array}{c}-2.3 \\
{[-13 ;+42]}\end{array}$ & $\begin{array}{c}+26 \\
{[-7.8 ;+63]}\end{array}$ & $\begin{array}{c}-6.8 \\
{[-26 ;+3.3]}\end{array}$ & $\begin{array}{c}+1.4 \\
{[-18 ;+22]}\end{array}$ & $\begin{array}{c}-0.3 \\
{[-13 ;+10]}\end{array}$ & $\begin{array}{c}+15 \\
{[-3.6 ;+47]}\end{array}$ & 0.1854 \\
\hline ETP (\%) & 0 & $\begin{array}{c}+8.4 \\
{[-47 ;+17]}\end{array}$ & $\begin{array}{c}-4.0 \\
{[-14 ;+8.7]}\end{array}$ & $\begin{array}{c}+4.4 \\
{[-17 ;+26]}\end{array}$ & $\begin{array}{c}-6.5 \\
{[-29 ;+0.9]}\end{array}$ & $\begin{array}{c}-3.9 \\
{[-28 ;+9.8]}\end{array}$ & $\begin{array}{c}+6.3 \\
{[-0.1 ;+11]}\end{array}$ & $\begin{array}{c}+12 \\
{[-2.5 ; 23]}\end{array}$ & 0.2950 \\
\hline \multicolumn{10}{|c|}{ E. CTI-PPP 1 pM TF } \\
\hline Peak (\%) & 0 & $\begin{array}{c}+17 * \\
{[+9.2 ;+55]}\end{array}$ & $\begin{array}{c}-8.2 \\
{[-17 ;-0.8]}\end{array}$ & $\begin{array}{c}+6.9 \\
{[-4.8 ;+70]}\end{array}$ & $\begin{array}{c}+6.4 \\
{[-11 ;+27]}\end{array}$ & $\begin{array}{c}+15^{*} \\
{[+6.3 ;+29]}\end{array}$ & $\begin{array}{c}+14 \\
{[+0.7 ;+23]}\end{array}$ & $\begin{array}{c}+18 \\
{[+6.0 ; 71]}\end{array}$ & 0.0125 \\
\hline ETP (\%) & 0 & $\begin{array}{c}+12 \\
{[+3.9 ;+65]}\end{array}$ & $\begin{array}{c}+1.1 \\
{[-10.03 ;+25]}\end{array}$ & $\begin{array}{c}+4.1 \\
{[-2.6 ;+65]}\end{array}$ & $\begin{array}{c}+6.8 \\
{[-8.2 ;+44]}\end{array}$ & $\begin{array}{c}+10 \\
{[+3.1 ;+43]}\end{array}$ & $\begin{array}{c}+18 * \\
{[+7.0 ;+44]}\end{array}$ & $\begin{array}{c}+11^{*} \\
{[+0.9 ;+62]}\end{array}$ & 0.0970 \\
\hline \multicolumn{10}{|l|}{ F. PPP 5 pM TF } \\
\hline Peak (\%) & 0 & $\begin{array}{c}+18 * \\
{[+13 ;+36]}\end{array}$ & $\begin{array}{c}-0.1 \\
{[-7.0 ;+11]}\end{array}$ & $\begin{array}{c}+9.1 \\
{[+0.1 ;+22]}\end{array}$ & $\begin{array}{c}-1.1 \\
{[-10 ;+8.2]}\end{array}$ & $\begin{array}{c}+18 * \\
{[+3.1 ;+39]}\end{array}$ & $\begin{array}{c}+10 \\
{[-2.7 ;+24]}\end{array}$ & $\begin{array}{c}+17.2 * \\
{[+6.0 ;+32]}\end{array}$ & 0.0013 \\
\hline ETP (\%) & 0 & $\begin{array}{c}-2.0 \\
{[-5.9 ;+3.1]}\end{array}$ & $\begin{array}{c}+6.8^{*} \\
{[+4.9 ;+12]}\end{array}$ & $\begin{array}{c}-2.0 \\
{[-6.0 ;+3.1]}\end{array}$ & $\begin{array}{c}+11 \\
{[+2.4 ;+14]}\end{array}$ & $\begin{array}{c}+7.6 \\
{[+1.6 ;+14]}\end{array}$ & $\begin{array}{c}+11 * \\
{[+7.5 ;+13]}\end{array}$ & $\begin{array}{c}+11 * \\
{[+3,0 ;+14]}\end{array}$ & 0.0027 \\
\hline
\end{tabular}

(Caption Table S2). TG was measured before and after 2 hours of strenuous exercise, once at 50 $\mathrm{m}$ altitude and three times at 3,375 $\mathrm{m}$ altitude. $\mathrm{N}=6,1$ measurement is missing (subject 5, WB TC post 3). Results are expressed as the absolute change compared to the value at $50 \mathrm{~m}$ pre-exercise; a value above 0 indicates an increase, a value below 0 indicates a decrease. Data are presented as median [ $25^{\text {th }}$ percentile; $75^{\text {th }}$ percentile], $*=p<0.05$ compared to zero (Wilcoxon signed ranks test). P-value $=$ Friedman's test. A. Thrombin generation (TG) in whole blood (WB) without added tissue factor (TF). Parameters derived from the TG curve are peak height (Peak in $\mathrm{nM}$ ) and endogenous thrombin potential (ETP in nM.min). B. TG in WB triggered with 0.5 pM TF. Parameters derived from the TG curve are Peak in \% of normal pool plasma (NPP) and ETP in \% of NPP. C. TG in platelet-rich plasma (PRP) triggered with 1 pM TF. Parameters derived from the TG curve are Peak in \% of NPP and ETP in \% of NPP. D. TG in platelet poor plasma (PPP) triggered with 1 pM TF. Parameters derived from the TG curve are Peak in \% of NPP and ETP in \% of NPP. E. TG in PPP anticoagulated with corn trypsin inhibitor (CTI) triggered with 1 pM TF. Parameters derived from the TG curve are Peak in \% of NPP and ETP in \% of NPP. F. TG in PPP triggered with 5 PM TF. Parameters derived from the TG curve are Peak in \% of NPP and ETP in \% of NPP. 


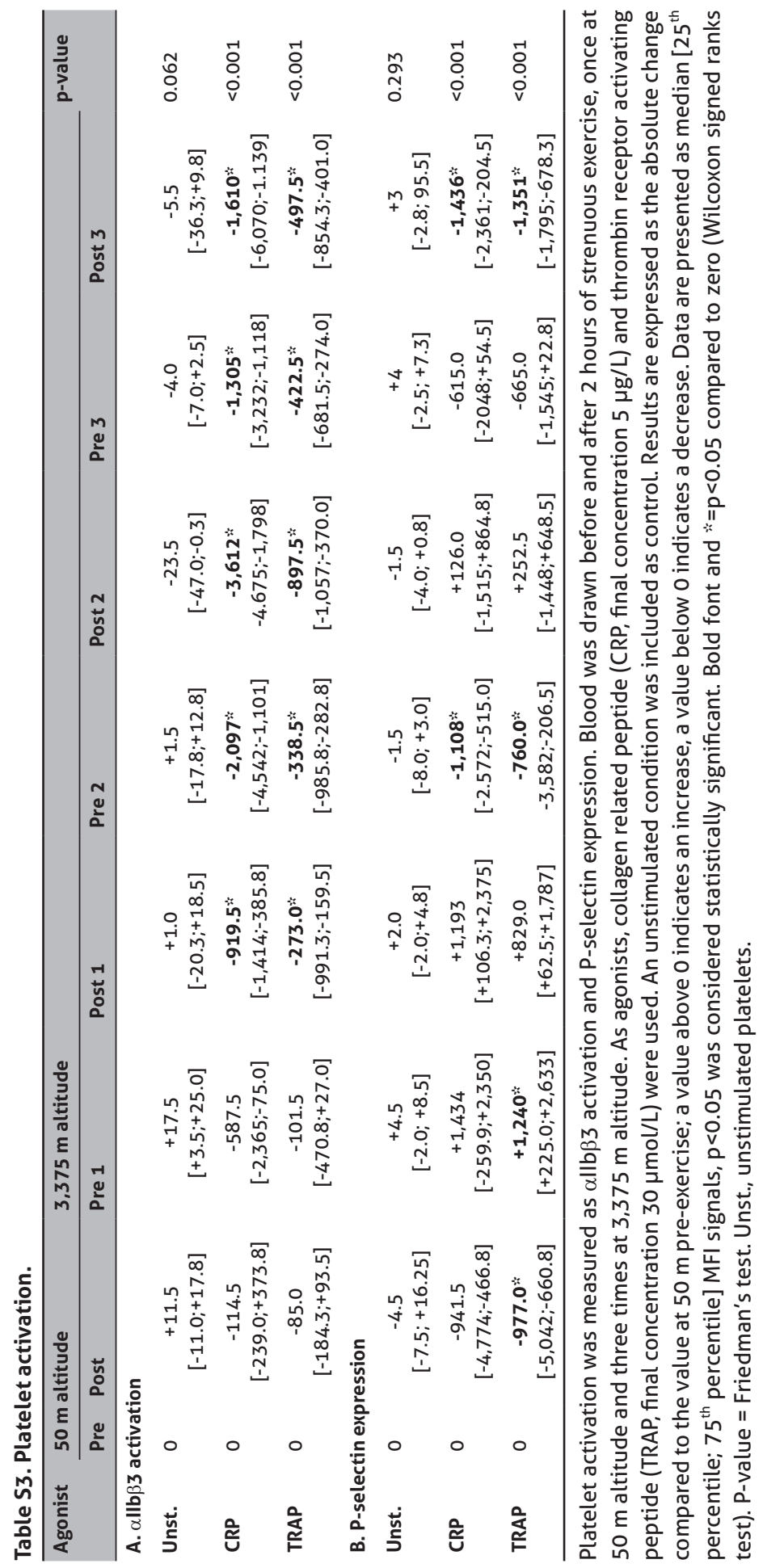



Part III 


\section{Active VWF in pathologies associated with unbalanced haemostasis}




$$
\text { Chapter } 6
$$




\section{Acute exacerbations of COPD}

induce a prothrombotic state through PMCs, endothelial activation and increased thrombin generation

Van der Vorm LN, Li L, Huskens D, Hulstein JJJ, Roest M, de Groot PG, ten Cate $H$, de Laat $B$, Remijn JA, Simons SO 


\section{ABSTRACT}

Rationale - Patients with chronic obstructive pulmonary disease (COPD) are at increased risk for cardiovascular events, particularly following an acute exacerbation (AE-COPD). Exacerbations are associated with increased systemic inflammation, which may drive coagulation.

Objectives - This prospective cohort study aimed to determine the effects of an AECOPD on platelet activation, the endothelium and plasmatic coagulation, and its association with systemic inflammation.

Methods - Fifty-two patients with an AE-COPD were included. Blood samples at admission, at day 3 of treatment and at convalescence were available for 32 patients. Platelet-monocyte complex (PMC) formation, monocyte Mac-1 expression and platelet (re)activity (P-selectin expression, GPIIbllla activation) were measured by flow cytometry. Von Willebrand Factor (VWF) and thrombin generation (TG) were determined as measures of endothelial activation and plasmatic coagulation, respectively.

Results - Exacerbations were associated with increased PMCs (MFI 31.3 vs 23.8, $\mathrm{p}=0.004$ ) and Mac-1 (MFI 38.2 vs 34.8, $\mathrm{p}=0.006$ ) compared to convalescence, but not with changes in platelet (re)activity. VWF (antigen, activity, active fraction) and TG (peak, ETP and velocity index) were all significantly higher during AE-COPD compared to convalescence. PMCs, Mac-1, VWF and TG were positively associated with systemic inflammation (CRP). Moreover, platelet hyperreactivity on admission was associated with an increased risk for exacerbation relapse.

Conclusion - Acute exacerbations induce an inflammation-associated prothrombotic state, characterized by increased PMCs, endothelial activation and plasmatic coagulation. Our findings provide direction for future studies on biomarkers predicting the risk of exacerbation relapse and cardiovascular events. 


\section{INTRODUCTION}

Cardiovascular disease (CVD) is the most important comorbidity associated with chronic obstructive pulmonary disease (COPD) due to its impact on overall prognosis, including mortality. Importantly, the prevalence of thrombotic events, such as myocardial infarction, stroke and pulmonary embolism, is known to increase during and shortly after acute exacerbations of COPD (AE-COPD), but the precise mechanisms remain unclear. ${ }^{2-4}$

COPD exacerbations are most frequently caused by viral and/or bacterial infections. ${ }^{5}$ The inflammatory response that is needed to control the infection, might also induce coagulation during an AE-COPD. ${ }^{6}$ First, inflammatory stimuli induce platelet activation, resulting in glycoprotein (GP)Ilbllla (fibrinogen receptor) activation, thereby mediating platelet adhesion and aggregation ${ }^{7,8}$ Activated platelets also secrete procoagulant substances, such as ADP and fibrinogen, as well as P-selectin. ${ }^{7}$ P-selectin can bind to its counterreceptor P-selectin glycoprotein ligand-1 (PSGL-1), constitutively expressed on the membrane of monocytes. Binding of platelets to monocytes results in the expression of the monocyte integrin Mac- $1 .{ }^{9}$ Several studies have demonstrated the importance of these platelet-monocyte complexes (PMCs) as a marker of platelet activation $^{8,10}$, but also as an early process in the pathogenesis of atherothrombosis. ${ }^{11-13}$ Second, inflamed endothelium releases von Willebrand Factor (VWF), which mediates adhesion of platelets to the vessel wall and subsequent thrombus growth. ${ }^{14}$ Third, inflammation also promotes thrombin generation (TG). Thrombin is the key effector of plasmatic coagulation, but also has an array of effects on endothelial cells, monocytes, and platelets. ${ }^{15}$

The interplay between inflammation and coagulation during AE-COPD suggests that activation of platelets, the endothelium and the plasmatic coagulation may all play a role in the pathogenesis of CVD in these patients. Few studies investigated platelet activation during $A E-C O P D$, and found increased PMCs without changes in platelet Pselectin expression. ${ }^{16,17}$ In addition, increased VWF antigen (VWF:Ag) levels and VWF ristocetin cofactor activity (VWF:RCo) have been reported in AE-COPD patients. ${ }^{18} \mathrm{Ac}$ tive $\mathrm{VWF}^{19}$ and VWF propeptide (VWFpp) ${ }^{20}$ provide additional information about VWF processing and the chronicity of endothelial activation, but these have not been studied in AE-COPD. Lastly, one study found increased thrombin-antithrombin complex (TAT), a surrogate marker of thrombin formation during $A E-C O P D^{21}$, but full thrombin generation profiles have not been determined in AE-COPD.

Together, platelets, endothelium and circulating coagulation factors determine the overall thrombotic tendency, but they have been scarcely studied together in AE-COPD 
patients. Therefore, the current study aimed to determine the effects of an AE-COPD on platelet activity and PMC formation, endothelial function and plasmatic coagulation. We hypothesized that during an AE-COPD all of these factors are in a heightened state of activation, and that these changes are associated with increased systemic inflammation.

\section{METHODS}

\section{Study subjects and design}

In this prospective observational cohort study, we recruited 52 subjects with a confirmed diagnosis of an AE-COPD (in accordance with the GOLD 2019 definition ${ }^{22}$ ), admitted to the Department of Respiratory Medicine of the Gelre Hospitals Apeldoorn, the Netherlands, between December 2018 and June 2019. Inclusion criteria were: (1) age $\geq 40$ years, ( 2 ) current or former smoker ( $\geq 10$ pack-years) (3) moderate to severe COPD (GOLD $\geq 2$ ) and (3) hospital admission due to an AE-COPD. An AE-COPD was defined as an acute worsening of respiratory symptoms requiring additional therapy. ${ }^{22}$ Exclusion criteria were: use of platelet function inhibitors (therapy with acetylsalicylic acid was allowed), vitamin $\mathrm{K}$ antagonists or direct oral anticoagulants; concomitant diagnosis of asthma, hepatic and/or renal failure, malignancies (excluding basal cell carcinoma) and chronic inflammatory diseases. Eligible patients were asked to participate within $24 \mathrm{~h}$ after admission. All patients received exacerbation treatment in accordance with national Dutch guidelines and were treated with steroids (100\%) and antibiotics $(27 \%)$. The majority of patients $(87 \%)$ also received prophylactic low molecular weight heparin on admission based on current guidelines on antithrombotic therapy.

\section{Ethics}

Patients were included after written informed consent, complying with the Declaration of Helsinki (version 2013). This study was approved by the Medical Research Ethics Committee of Maastricht University, the Netherlands (METC azM/UM, reference NL66067.068.18). The inclusion procedure is depicted in Figure 1.

\section{Blood collection and processing}

Blood samples were obtained during AE-COPD (within 24h of admission, "AE-COPD"), 3 days later ("day 3 of treatment") and (when clinically stable) on average 8 weeks (min 6, max 12 weeks) after discharge ("convalescence"). Venous blood was collected by venipuncture into EDTA and 3.2\% sodium citrate vacuum tubes (BD Vacutainer System, Franklin Lakes, USA). Blood cell counts, routine coagulation tests (additional detail on these tests and VWF assays is provided in the Supplemental Methods) and flow 


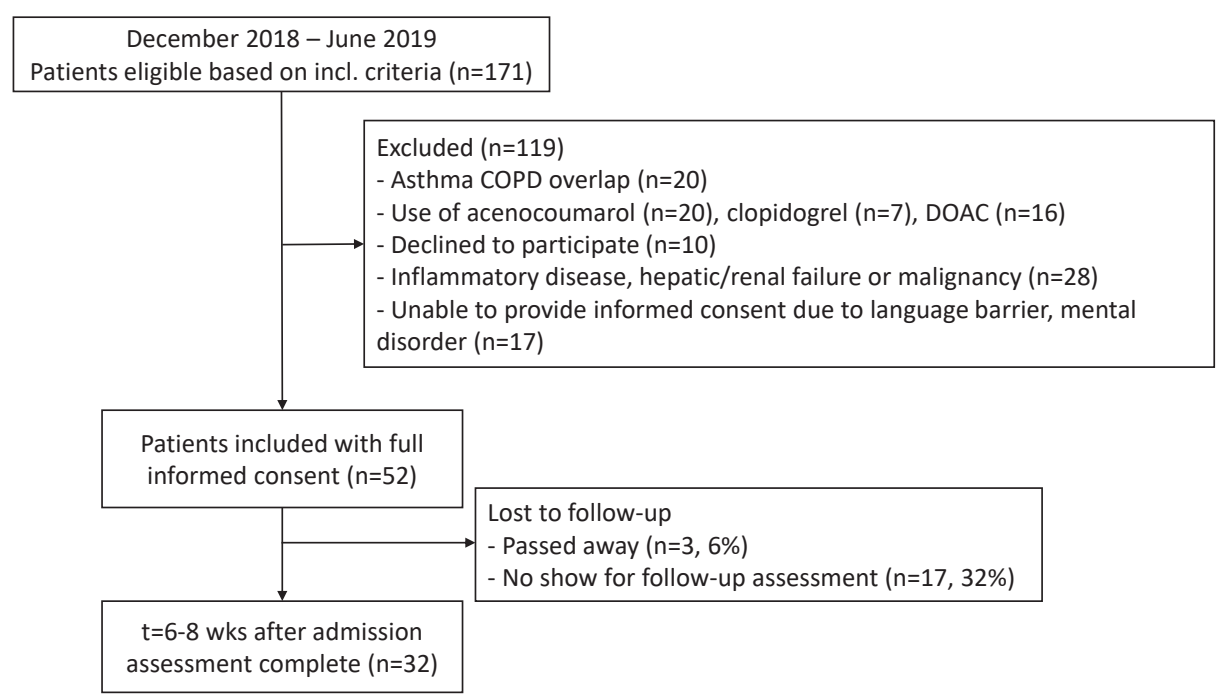

Figure 1. Inclusion flow-chart.

cytometric assays were performed within 4 hours after blood collection. The remaining blood was centrifuged twice at $2,840 \mathrm{~g}$ for 10 minutes. Platelet-poor plasma (PPP) was stored at $-80^{\circ} \mathrm{C}$ until further analyses.

\section{PMCs and Mac-1 expression}

Whole blood $(20 \mu \mathrm{L})$ was incubated for 15 minutes with a $20 \mu \mathrm{L}$ reaction mixture consisting of monoclonal antibodies against monocyte CD14 (APC-conjugated) (Becton Dickinson) and platelet GPIIbllla (anti-CD41a PE-conjugated) for PMCs or antibodies against monocyte CD14 (APC-conjugated) and CD11b (FITC-conjugated) for Mac-1 expression. Subsequently, BD FACS ${ }^{\mathrm{TM}}$ Lysing solution (Becton-Dickinson, diluted 10-fold with millio) was added, vortexed and incubated for 10 minutes at room temperature. Samples were stored at $4^{\circ} \mathrm{C}$ until analysis (on the same day) by flow cytometry (Navios, Beckman Coulter, Woerden, the Netherlands). Gating was performed as described previously. ${ }^{16}$

\section{Platelet activation and reactivity}

Flow cytometric analysis of the activation of GPIIbllla and expression of P-selectin (CD62P) was used to determine platelet activation in response to thrombin-activated peptide (TRAP), collagen-related peptide and adenosine diphosphate (ADP), as described previously. ${ }^{23}$ Results are expressed as median fluorescent intensity (MFI), calculated by subtracting the MFI of the unstimulated control from the MFI of the condition with agonist. 


\section{Plasmatic coagulation}

Calibrated automated thrombinography (CAT) TG in PPP was performed as described previously. ${ }^{24}$

\section{Statistical analysis}

Conformity to the normal distribution was evaluated with Q-O. plots and the ShapiroWilk test. Categorical variables were expressed as percentages and continuous variables as mean $\pm \mathrm{SD}$ or median (interquartile range, IQR). Comparisons between the three time-points (for 32 patients with complete follow-up) were performed by repeatedmeasures ANOVA with post-hoc Bonferroni test or non-parametric Friedman tests with post-hoc Wilcoxon signed-rank tests. Comparisons between two independent groups (at baseline, all 52 subjects) were performed by independent samples t-test or nonparametric Mann-Whitney $U$ test. Associations were assessed using Spearman's rank correlation tests. A p-value of 0.05 was considered statistically significant. All analyses were performed using Statistical Package for Social Sciences (SPSS Inc., Chicago, IL, USA) version 25 .

\section{RESULTS}

\section{Baseline characteristics of the study population}

Fifty-two patients admitted to the Gelre Hospitals Apeldoorn with a confirmed AE-COPD were included in this study. Three patients (6\%) died during the follow-up as a consequence of respiratory failure. Moreover, 17 patients did not show up for the blood draw at convalescence (on average 8 weeks after discharge). Thus, blood samples at all 3 time points were obtained for 32 subjects (Figure 1, Table S1). Baseline characteristics of the study participants are summarized in Table 1 . In approximately half of the patients, the AE-COPD had a proven infectious cause. At presentation, 26 of the patients had CRP levels $>10 \mathrm{mg} / \mathrm{L}$, indicative of systemic inflammation, of which 18 had a proven viral $(n=6)$, bacterial $(n=9)$ or combined $(n=3)$ infectious cause. Fifteen patients $(28.8 \%)$ had a history of CVD, for which they all received aspirin.

\section{Increased PMC formation and monocyte activation during AE-COPD}

PMCs were significantly increased during AE-COPD (median 31.3, IOR 15.3) compared to after three days of treatment (median 20.8, IOR 8.2, $\mathrm{p}<0.001$ ) and convalescence (median 23.8, IOR 7.9, $\mathrm{p}=0.004$ ) (Figure 2A). There was a small, positive correlation between PMCs and CRP levels (Figure 2B, open circles) 
Table 1. Baseline patient characteristics.

\begin{tabular}{|c|c|}
\hline Variable & Mean \pm SD; Median (IQR); $n$ [\%] \\
\hline $\mathrm{N}$ & 52 \\
\hline Age, years & $66.5 \pm 10.4$ \\
\hline Male, $n[\%]$ & $21[40.4 \%]$ \\
\hline Body mass index, $\mathrm{kg} / \mathrm{m}^{2}$ & $25.1(5.3)$ \\
\hline Current smoker, $n$ [\%] & $16[30.8 \%]$ \\
\hline Smoking history, pack-years & $31.5 \pm 12.9$ \\
\hline \multicolumn{2}{|l|}{ GOLD score, $n[\%]$} \\
\hline II & $15[28.8 \%]$ \\
\hline III & $22[42.3 \%]$ \\
\hline IV & $15[28.8 \%]$ \\
\hline \multicolumn{2}{|l|}{ Pulmonary function } \\
\hline FEV $1, l$ & $0.92(0.53)$ \\
\hline FEV1 predicted, \% & $36.0(17.4)$ \\
\hline VC, $l$ & $2.5 \pm 0.9$ \\
\hline VC predicted, \% & $74.6 \pm 18.7$ \\
\hline Tiffeneau index, \% & $37.0(16.0)$ \\
\hline \multicolumn{2}{|l|}{ Inhalation medication, $n[\%]$} \\
\hline Short-acting bronchodilators (SAMA/SABA) & $45[86.5 \%]$ \\
\hline Monotherapy long-acting (LABA/LAMA) & $7[13.5 \%]$ \\
\hline Double therapy (LABA+LAMA/LABA+ICS) & $16[30.8 \%]$ \\
\hline Triple therapy (LABA+LAMA+ICS) & $25[48.1 \%]$ \\
\hline Emphysema, $n[\%]$ & $34[65.4 \%]$ \\
\hline Exacerbation rate, $n$ per year & $2(2)$ \\
\hline Нурохетіа* at presentation, $n$ [\%] & $19[36.5 \%]$ \\
\hline CRP $>10 \mathrm{mg} / \mathrm{L}$ at presentation, $n$ [\%] & $26[50.0 \%]$ \\
\hline Infectious cause of AE-COPD, $n[\%]$ & $27[51.9 \%]$ \\
\hline Viral infection & $12[23.1 \%]$ \\
\hline Bacterial infection & $10[19,2 \%]$ \\
\hline Combined bacterial and viral infection & $5[9.6 \%]$ \\
\hline Duration of hospitalization, days & $6(4)$ \\
\hline LMWH during hospital stay, $n$ [\%] & $45[86.5 \%]$ \\
\hline History of $\mathrm{CVD}^{\dagger}, n[\%]$ & $15[28.8 \%]$ \\
\hline On aspirin therapy, $n[\%]$ & $15[28.8 \%]$ \\
\hline
\end{tabular}

Data are presented as the mean \pm SD or median $(I Q R)$ for normally distributed and skewed continuous variables, respectively, and as the absolute number [percentage of total] for categorical variables. " Hypoxemia was defined as oxygen saturation $<90 \%$ while breathing room air. $\dagger$ Including deep venous thrombosis, ischemic stroke, transient ischemic attack, pulmonary embolism, acute coronary syndrome, heart failure. Abbreviations: FEV1, forced expiratory volume in 1 second; VC, vital capacity; LMWH: prophylactic low-molecular-weight heparin; SAMA/LAMA, short-acting/longacting muscarinic antagonist; SABA/LABA, short-acting/long-acting beta-agonists; ICS, inhaled corticosteroids; CRP, C-reactive protein; AE-COPD, acute exacerbation of COPD; CVD, cardiovascular disease. 

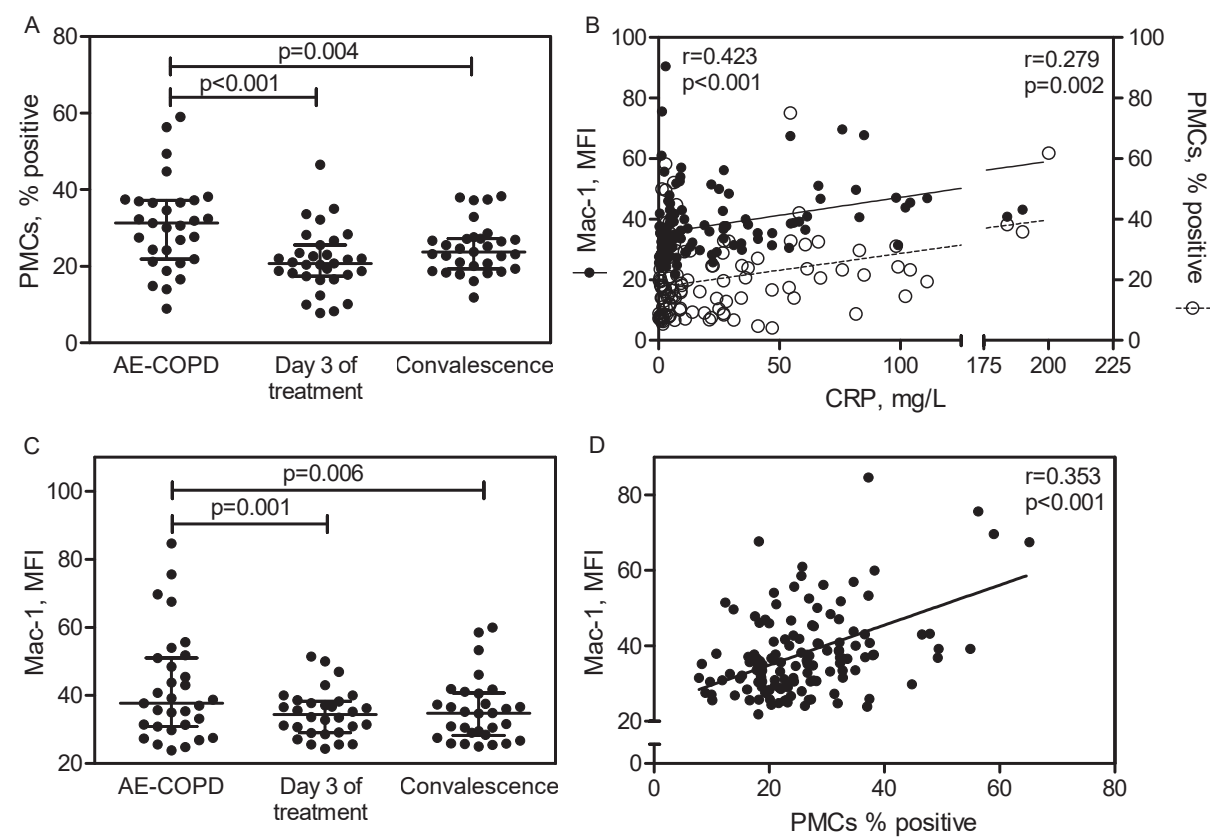

Figure 2. Platelet-monocyte complexes and monocyte Mac-1 expression during and after AECOPD. Circulating PMCs (A) and Mac-1 expression (C) were measured at the 3 time points. The horizontal line represents the median, whiskers indicate the first and third quartiles. Significant differences are indicated with corresponding p-values. (B) The correlation between PMCs (right axis, open circles with dashed linear regression) and Mac-1 expression (left axis, closed circles with solid linear regression) with inflammation, measured as CRP. Corresponding Spearman correlation coefficients and p-values are indicated in the upper left and right corners for Mac-1 and PMCs, respectively. (D) Correlation between PMCs and Mac-1 expression (data from all 3 time points). Abbreviations: PMCs, platelet-monocyte complexes; MFI, median fluorescent intensity.

Monocyte Mac-1 expression was also increased during AE-COPD (median 38.2, IOR 20.0) compared to during treatment (median 34.0, IOQR 9.9, $p=0.001$ ) and convalescence (median 34.8, IQR 12.5, $\mathrm{p}=0.006$ ) (Figure 2C). There was a positive correlation between monocyte Mac-1 expression and PMC formation ( $r=0.353, p<0.001$ ) (Figure 2D). Patients with CRP levels $>10 \mathrm{mg} / \mathrm{L}$ at baseline had significantly higher monocyte Mac-1 expression (median MFI 38.7, IOR 14.5) than patients with CRP below this cut-off (33.5, IOR 11.4) $(p=0.002)$ and the correlation between CRP and Mac-1 was also significant $(r=0.423, p<0.001$ ) (Figure $2 B$, closed circles).

\section{Platelet P-selectin expression and GPIIbIlla activation not affected by AE-COPD}

P-selectin expression, either unstimulated or in response to TRAP, collagen-related peptide and ADP, was not significantly different between AE-COPD, day 3 of treatment and 
Table 2. Platelet (re)activity during and after AE-COPD.

\begin{tabular}{lllll}
\hline \multicolumn{1}{l}{ Parameter } & AE-COPD & Day 3 of treatment & Convalescence & P-value \\
\hline \multicolumn{2}{l}{ P-selectin expression (MFI) } & & & \\
Unstimulated & $0.32(0.07)$ & $0.32(0.08)$ & $0.32(0.12)$ & $\mathrm{ns}$ \\
TRAP & $26.2(8.6)$ & $25.0(10.1)$ & $23.9(6.0)$ & $\mathrm{ns}$ \\
CRP & $24.4(9.4)$ & $24.3(7.8)$ & $24.8(8.0)$ & $\mathrm{ns}$ \\
ADP & $5.8(8.0)$ & $5.6(5.1)$ & $6.4(7.2)$ & $\mathrm{ns}$ \\
GPIIbllla activation (MFI) & & & \\
Unstimulated & $0.53(0.30)$ & $0.57(0.27)$ & $0.64(0.27)$ & $\mathrm{ns}$ \\
TRAP & $2.6(2.2)$ & $2.1(2.6)$ & $3.2(2.7)$ & $\mathrm{ns}$ \\
CRP & $19.1(10.5)$ & $20.9(13.1)$ & $20.2(9.2)$ & $\mathrm{ns}$ \\
ADP & $13.3(7.6)$ & $11.9(9.2)$ & $13.6(11.0)$ & $\mathrm{ns}$ \\
\hline
\end{tabular}

P-selectin expression and GPIIbIlla activation were measured by flow cytometry as markers of platelet activation. Whole blood was incubated without (unstimulated, representing spontaneous/'in vivo" platelet activation) or with one of the platelet agonists TRAP, CRP or ADP. Data are presented as the median (IOR), $n=32$. Abbreviations: TRAP, thrombin receptor activating peptide; CRP, collagen-related peptide; ADP, adenosine diphosphate; MFI, median fluorescent intensity; ns, nonsignificant.

convalescence (Table 2, top half). Similarly, activation of the fibrinogen receptor GPIIbIlla was not significantly different between the 3 time points for the unstimulated condition nor for any of the tested agonists (Table 2, bottom half). In patients with CRP>10 mg/L at baseline, P-selectin expression in response to TRAP and collagen-related peptide were significantly higher ( $p=0.010$ and $p=0.007$, respectively) compared to patients without systemic inflammation (TRAP MFI 28.4 vs 24.1 and CRP MFI 26.7 vs 23.3, respectively).

\section{Endothelial activation during AE-COPD}

Endothelial function was evaluated by measuring various VWF parameters. VWF:Ag levels were significantly elevated during AE-COPD (median 218\%, IOR 113\%) and at day 3 of treatment (median $217 \%$, IOQ 126\%) compared to convalescence (median 182\%, IOR 110\%) (Figure 3A). Similarly, VWF:RCo activity was increased during AE-COPD (median 157\%, IOR 89\%, $p=0.009$ ) and day 3 of treatment (median 158\%, IOR 64\%, $\mathrm{p}=0.002$ ) compared to convalescence (median 135\%, IOR 71\%) (Figure 3B). Active VWF levels were highest during AE-COPD (median 151\%, IOR 48), followed by a drop during treatment (median 142\%, IOR 32, p<0.001) and lowest levels at convalescence (median $125 \%$, IOR 27, p<0.001) (Figure 3C). In contrast, VWFpp levels remained stable over time (Figure 3D). CRP correlated significantly with active VWF (Spearman $r=0.395, p<0.001$ ), VWF:Ag (Spearman $r=0.475, p<0.001$ ) and VWFpp (Spearman $r=0.312, p=0.001$ ) levels. 

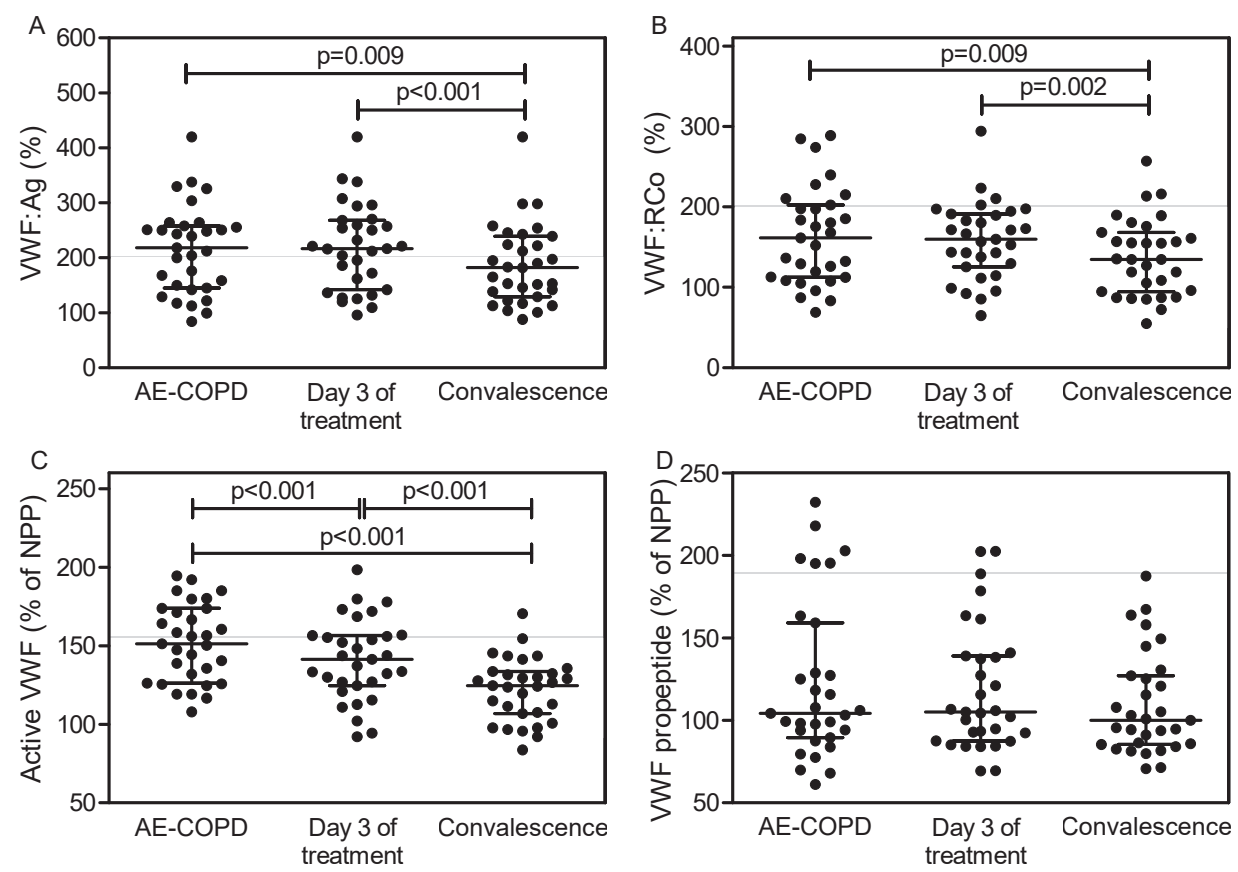

Figure 3. Endothelial cell activation measured by circulating VWF parameters. (A) VWF:Ag, (B) VWF:RCo and (C) active VWF at the 3 sample time points. The horizontal line represents the median, whiskers indicate the first and third quartiles. Significant differences are indicated with corresponding p-values. Abbreviations: NPP, normal pooled plasma; VWF:Ag, VWF antigen; VWF:RCo, VWF ristocetin cofactor activity; AE-COPD, acute exacerbation of COPD.

\section{Plasmatic coagulation is increased during AE-COPD}

The total (ETP) and maximum (peak) amount of thrombin generated and the velocity of thrombin formation ( $\mathrm{VI}$ ) were all significantly increased during AE-COPD compared to convalescence, at all concentrations ( 0,1 and $5 \mathrm{pM}$ ) of TF (Table 3, Figure 4A-C). The ETP (at 1 and 5 pM TF) was also increased during AE-COPD (1.502 \pm 356 and $1.503 \pm$ $335 \mathrm{nM}$.min, respectively) compared to day 3 of treatment (1.440 \pm 332 and $1.418 \pm$ 271 nM.min, respectively, both $\mathrm{p}<0.001$ ). The "time-parameters" lagtime and TTP were not significantly different between the timepoints. Of note, other global assays for plasmatic coagulation, the prothrombin time (PT) and activated partial thromboplastin time (aPTT), did not show any differences between the 3 time points (Table S2). CRP correlated significantly with the ETP (Spearman $r$ between 0.244 and 0.315 ), peak ( $r$ between 0.361 and 0.405 ) and velocity index ( $r$ between 0.364 and 0.450 ) of TG stimulated with 0, 1 and 5 pM (Table S3). In line with this, patients with CRP>10 mg/L had a significantly elevated peak, ETP and velocity index compared to those without systemic inflammation (Figure S1). 
Table 3. Thrombin generation during and after AE-COPD

\begin{tabular}{|c|c|c|c|c|}
\hline & AE-COPD & Day 3 treatment & Convalescence & p-value \\
\hline \multicolumn{5}{|l|}{5 pM TF } \\
\hline LT, s & $3.6(3.3-3.9)$ & $3.3(3.0-4.0)$ & $3.6(3.1-4.0)$ & ns \\
\hline ETP, nM.min & $1503 \pm 335 \%$ & $1418 \pm 271 \dagger$ & $1400 \pm 360 *$ & $\begin{array}{l}* p=0.015 \\
+p<0.001\end{array}$ \\
\hline Peak, $n M$ & $283(240-321) *$ & $260(230-308)$ & $251(209-290) *$ & $* p=0.003$ \\
\hline TTP, $s$ & $6.6(6.0-7.0)$ & $6.3(6.0-7.3)$ & $6.7(6.2-7.3)$ & ns \\
\hline $\begin{array}{l}\text { VI, } n M / \min \\
1 \text { pM TF }\end{array}$ & $101.3 \pm 39.0^{*}$ & $91.8 \pm 39.8$ & $80.2 \pm 36.1^{*}$ & $\Rightarrow p=0.01$ \\
\hline LT, s & $5.8(5.4-6.6)$ & $6.0(5.3-6.8)$ & $6.0(5.2-6.6)$ & ns \\
\hline ETP, nM.min & $1502 \pm 356 \%$ & $1440 \pm 332 \dagger$ & $1375 \pm 361^{*}$ & $\begin{array}{l}* p=0.008 \\
+p<0.001\end{array}$ \\
\hline Peak, $n M$ & $303(261-350) *$ & $278(233-328)$ & $265(218-291) *$ & $* p=0.001$ \\
\hline TTP, $s$ & $8.7(8.0-9.3)$ & $8.5(8.2-9.9)$ & $8.9(8.0-9.9)$ & ns \\
\hline VI, $n M / \min$ & $116.7 \pm 42.6^{*}$ & $107.4 \pm 48.8$ & $90.7 \pm 41.9^{*}$ & $* p=0.002$ \\
\hline $0 \mathrm{pM}$ TF & & & & \\
\hline LT, $s$ & $11.0(9.8-12.6)$ & $11.8(10.2-12.5)$ & $11.5(9.5-13.5)$ & ns \\
\hline ETP, nM.min & $1452 \pm 354^{*}$ & $1379 \pm 305^{*}$ & $1331 \pm 295$ & $* p<0.001$ \\
\hline Peak, $n M$ & $355(308-390) *$ & $335(281-386) \dagger$ & $315(264-347) * \dagger$ & $\begin{aligned} * p & =0.003 \\
t p & =0.016\end{aligned}$ \\
\hline TTP, $s$ & $13.0(12.0-15.0)$ & $13.8(12.3-15.2)$ & $13.9(11.5-15.8)$ & ns \\
\hline VI, $n M / \min$ & $162.7 \pm 48.1^{*}$ & $152.4 \pm 60.0 \dagger$ & $133.0 \pm 43.1 * \dagger$ & $\begin{aligned} * p & =0.003 \\
\dagger p & =0.008\end{aligned}$ \\
\hline
\end{tabular}

Data are presented as mean \pm SD for normally distributed variables and median $\left(25^{\text {th }}-75^{\text {th }}\right.$ quartile $)$ for non-parametric variables." $\dagger$ and $\S$ indicate the values that are significantly different from each other. Abbreviations: AE-COPD, acute exacerbation of COPD; TF, tissue factor; LT, lagtime; ETP, endogenous thrombin potential; TTP, time to thrombin peak; VI, velocity index; ns, non-significant at the $p=0.05$ level.

Fibrinogen was significantly elevated during AE-COPD (mean 4.8 \pm SD 1.5) compared to day 3 of treatment (mean 4.2 \pm SD 1.3) (Table S2). Levels of FVIII were highest at day 3 of treatment (median 198, IOR 87) compared to onset AE-COPD (median 185, IOQR 91) and convalescence (median 153, IOR 84). Fibrinogen and FVIII levels correlated with CRP (Spearman $r=0.722$ and 0.339 , respectively, both $p<0.001$ ) and were significantly increased in patients with signs of systemic inflammation (fibrinogen mean 5.4, SD 1.2 g/L; FVIII median 154\%, IOQR 78\%) compared to patients without systemic inflammation (fibrinogen mean 3.6, SD 0.7 g/L; FVIII median 205\%, IOQR 78\%). D-dimer levels, a marker of in vivo coagulation activation, were not different between the 3 time points (Table S2). 


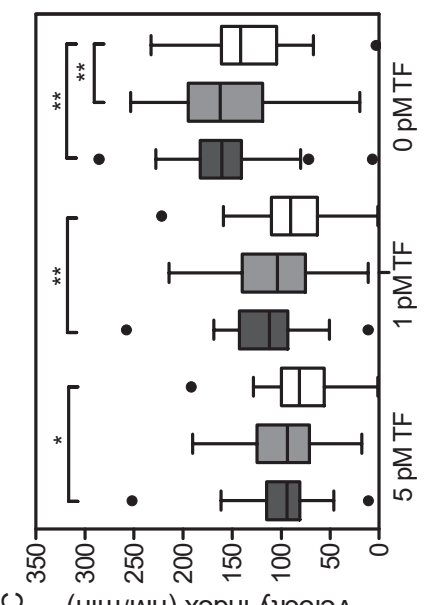

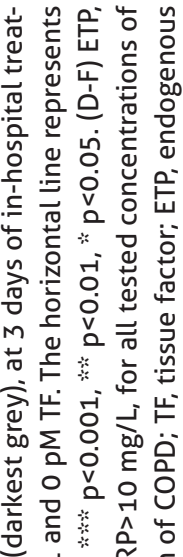

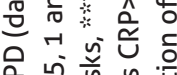

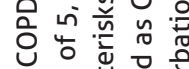
峞苍岕造

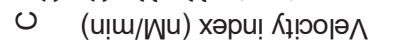

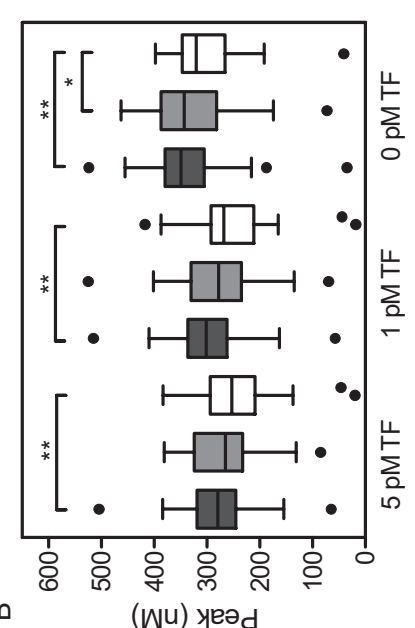

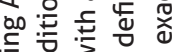

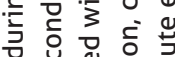
文苋苋 赵记 늘 들 은

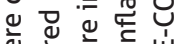
ऐ㐫岕

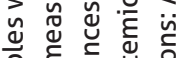

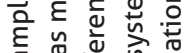

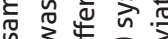

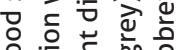

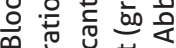

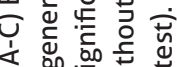

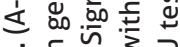

$\infty$ (wu) yeəন

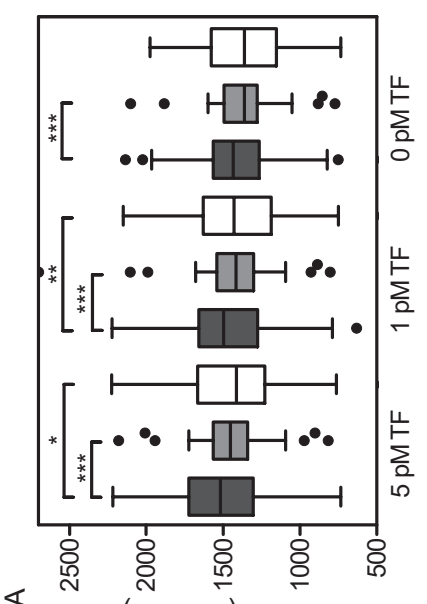
을 है 气ै

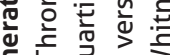

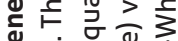
宁宁可

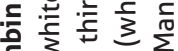
ह

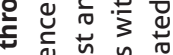

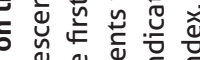
월. 이ㅇㅝㅛ 山己

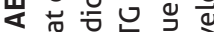

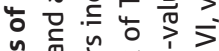
牙 ये क के

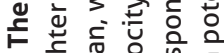
ن 는

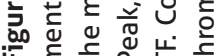




\section{Clinical outcomes in relation to coagulation parameters}

Three patients died during hospital admission. Clinical outcomes could be determined for all other 49 patients. Three patients suffered a cardiovascular event during the study: during admission, one patient suffered a non-ST segment elevation myocardial infarction and one had acute Takotsubo cardiomyopathy, while after discharge one patient had an unprovoked pulmonary embolism. No remarkable baseline (AE-COPD) coagulation parameter values were observed in these 3 patients, and given the small number of events we refrained from further post-hoc analyses.

Twenty-one patients suffered from a relapse of AE-COPD within the study follow-up period. Post-hoc analysis showed that platelet hyperreactivity at baseline, i.e. at hospital admission, was significantly associated with AE-COPD relapse after hospital discharge: patients who would later relapse had significantly higher platelet GPIIbllla activation in response to TRAP (MFI 7.9, IOR 4.8, $\mathrm{p}=0.001$ ), collagen-related peptide (MFI 44.8, IOQR 21.0, $p=0.007$ ) and ADP (MFI 31.6, IOR 11.1, p=0.017) at hospital admission compared to patients who did not have a relapse during the follow up (TRAP MFI 4.5, IOR 3.8; CRP MFI 35.4, IOR 24.8; ADP MFI 23.6, IOQR 10.9).

\section{DISCUSSION}

Acute exacerbations of COPD induce a prothrombotic state that is characterized by both platelet- and endothelial activation, as well as by an increase in plasmatic coagulation potential. Moreover, activation of these coagulation parameters was associated with increased systemic inflammatory markers, suggesting that both inflammation and coagulation are driving the prothrombotic state in AE-COPD.

Despite the evidence that AE-COPDs are associated with an increased risk of arterial and venous thrombotic events ${ }^{1,25}$, there are surprisingly few studies directly linking $A E-C O P D$ to the function and activation status of the coagulation system. In one prospective cohort study, Wedzicha et al. found that fibrinogen levels are elevated in stable COPD and further increase during an exacerbation. ${ }^{26}$ Two smaller studies have shown an increase in circulating platelet-monocyte complexes (PMCs) during an AE-COPD. ${ }^{16,17}$ Our study extends these results by showing that an AE-COPD is a "pan-thrombotic" state in which platelets, monocytes, the endothelium and plasmatic coagulation are all activated. Our finding that activated VWF is elevated during an AE-COPD is especially relevant since active VWF can directly interact with platelets, and is therefore highly thrombogenic. The observed simultaneous activation of the whole coagulation system 
supports the hypothesis that an AE-COPD itself is a thrombogenic trigger and thereby increases the risk of both arterial and venous thromboembolic events.

On the other hand, the coagulation system might potentially contribute to the occurrence of COPD exacerbations. Some studies have shown that even at baseline there might be some activation of the coagulation system in patients with stable COPD. ${ }^{27,28}$ It is hypothesized that platelet- and endothelial activation might affect COPD progression and exacerbation rate by impairing microvascular pulmonary blood flow and maintaining inflammation in COPD. ${ }^{29}$ In the MESA and SPIROMICS cohort studies chronic aspirin use was found to attenuate emphysema progression ${ }^{30}$ and was associated with a lower risk of exacerbations. ${ }^{31}$ This is in line with our observation of increased VWF:Ag but stable VWFpp, which suggests chronic, rather than acute, endothelial activation. ${ }^{20}$ Chronic endothelial activation in early adulthood has been associated with future emphysema development. ${ }^{32}$ Moreover, we report significantly higher platelet reactivity during the index exacerbation in patients who later suffer from a recurrent exacerbation. Given the post-hoc nature of this analysis and potential index event bias, this finding should be interpreted prudently.

We profiled thrombin generation to assess changes in plasmatic coagulation during an AE-COPD. Thrombin peak, ETP and velocity of thrombin generation were significantly increased during AE-COPD, while D-dimer levels, a marker of in vivo coagulation activation, did not change. In addition, the time until clot formation, reflected by the aPTT, PT and TG lagtime, were comparable at all time points. Together, this implies that the increased thrombin generation potential mainly serves to stabilize clots. Increased peak TG has been associated with subsequent risk of VTE, independent of established VTE risk factors. ${ }^{33}$ TC peak, ETP and velocity significantly correlated with inflammation, possibly through the inflammatory cytokine IL-6, which stimulates fibrinogen and FVIII release. ${ }^{34,35}$ Elevated FVIII and fibrinogen levels during AE-COPD contribute to the increased TG. ${ }^{26,36}$ Moreover, Mac-1 on activated monocytes catalyses the conversion of FX to FXa, which also boosts TG. ${ }^{37}$ Finally, the high TG peak and velocity index in the absence of added TF indicate that in vivo processes increase the availability of TF. These processes are likely linked to systemic inflammation, as IL-6 and CRP induce TF expression on monocytes, and apoptosis of several cell types increases circulating TF-bearing microparticles. ${ }^{38}$ These results underscore the close relationship between inflammation and coagulation in the occurrence of thromboembolic events after an AE-COPD.

Contrary to our hypothesis, we did not find "in vivo" platelet activation and hyperreactivity as measured by GPIIbllla and P-selectin expression, either unstimulated or after stimulation with platelet agonists, respectively (Table 2). PMC formation may 
be a more sensitive marker of platelet activation, as circulating platelets rapidly shed surface P-selectin but continue to circulate in complex with monocytes. ${ }^{10}$ The fact that we also found an increased mean platelet volume (Table S1), another surrogate marker of platelet activation, is in line with the increased PMC formation and supports that platelet activation did occur during AE-COPD. However, other mechanisms such as monocyte activation as the primary driver for PMC formation in COPD should be considered. We observed significantly increased monocyte Mac-1 expression during AE-COPD compared to convalescence, which correlated with PMC formation. Mac-1 expression is predominantly triggered by interaction with platelets through P-selectin-PSGL-1 binding but can also occur in response to inflammatory mediators such as CRP ${ }^{37}$, which was significantly increased during AE-COPD and correlated positively with both Mac-1 expression and circulating PMCs. This would support the hypothesis that inflammationassociated monocyte activation plays a key role in the PMC formation during AE-COPD.

Although the strengths of this study include the careful and comprehensive assessment of prothrombotic markers during an AE-COPD and after convalescence, several limitations should be discussed. First, our study sample was relatively small and lost-tofollow-up was larger than expected, which in part reflects the labour-intensive nature of such mechanistic studies. Nonetheless, due to the large changes in prothrombotic markers from exacerbation to convalescence, the study was still sufficiently powered to detect statistically significant changes in prothrombotic markers. Regrettably, we could not detect correlations between prothrombotic markers and the occurrence of cardiovascular outcome, nor could we find clinical risk factors for the increase in thrombotic markers. This is not surprising since our study was not set up and powered as such. Nevertheless, the large changes in thrombotic biomarkers seen in this study warrant future investigations into their relationship with the incidence of cardiovascular events. Our data suggest that active VWF, PMCs, and TG could be candidate biomarkers in such future studies. Second, the uniqueness of AE-COPD as a thrombogenic trigger should not be overestimated. Epidemiological studies in non-COPD populations have yielded similar results, demonstrating an increased cardiovascular risk following acute infections. ${ }^{39}$ Since COPD patients are more susceptible to infections, it might not be an intrinsic characteristic of COPD patients to develop thrombosis, but rather a multitude of infectious insults that initiates the thrombotic cascade. Third, we included 3 timepoints in our study, whereas longer, sequential time points might have been preferable to draw conclusions on the causality of prothrombotic changes seen during an AE-COPD and the subsequent risk of CVD.

In conclusion, elevated circulating PMCs, (active) VWF and thrombin generation together contribute to the prothrombotic state of COPD patients during an acute exacerbation, 
particularly in patients with increased systemic inflammation. Moreover, platelet hyperreactivity was associated with exacerbation relapse. These findings signify that AE-COPDs are "pan-thrombotic" events, but also suggest a reciprocal cause and effect in which increased platelet hyperreactivity may contribute to recurrent COPD exacerbations. The current study may therefore provide a basis for future studies with the aim to predict which $\mathrm{AE}-\mathrm{COPD}$ patients have the highest risk of developing a cardiovascular event.

\section{ACKNOWLEDGMENTS}

The authors would like to express their gratitude to all colleagues of the Department of Clinical Chemistry and Haematology of the Gelre Hospitals Apeldoorn for their indispensable help with blood drawings and their technical support in the laboratory. Furthermore, we would like to thank all colleagues of the Department of Respiratory medicine of the Gelre Hospitals Apeldoorn for their help during the inclusion phase of the study. 


\section{REFERENCES}

1. Anthonisen NR, Connett JE, Enright PL, Manfreda J, Lung Health Study Research G. Hospitalizations and mortality in the Lung Health Study. Am J Respir Crit Care Med. 2002;166(3):3339.

2. Donaldson GC, Hurst JR, Smith CJ, Hubbard RB, Wedzicha JA. Increased risk of myocardial infarction and stroke following exacerbation of COPD. Chest. 2010;137(5):1091-7.

3. Maclay JD, McAllister DA, Macnee W. Cardiovascular risk in chronic obstructive pulmonary disease. Respirology. 2007;12(5):634-41.

4. Rothnie KJ, Yan R, Smeeth L, Quint JK. Risk of myocardial infarction (MI) and death following $\mathrm{MI}$ in people with chronic obstructive pulmonary disease (COPD): a systematic review and meta-analysis. BMJ Open. 2015;5(9):e007824.

5. Papi A, Bellettato CM, Braccioni F, Romagnoli M, Casolari P, Caramori G, et al. Infections and airway inflammation in chronic obstructive pulmonary disease severe exacerbations. Am J Respir Crit Care Med. 2006;173(10):1114-21.

6. Thomsen $M$, Ingebrigtsen TS, Marott JL, Dahl M, Lange $P$, Vestbo J, et al. Inflammatory biomarkers and exacerbations in chronic obstructive pulmonary disease. JAMA. 2013;309(22):2353-61.

7. Malerba M, Clini E, Malagola M, Avanzi GC. Platelet activation as a novel mechanism of atherothrombotic risk in chronic obstructive pulmonary disease. Expert Rev Hematol. 2013;6(4):475-83.

8. Davi G, Patrono C. Platelet activation and atherothrombosis. The New England journal of medicine. 2007;357(24):2482-94.

9. Wang Y, Sakuma M, Chen Z, Ustinov V, Shi C, Croce K, et al. Leukocyte engagement of platelet glycoprotein Ibalpha via the integrin Mac-1 is critical for the biological response to vascular injury. Circulation. 2005;112(19):2993-3000.

10. Michelson AD, Barnard MR, Krueger LA, Valeri CR, Furman MI. Circulating monocyte-platelet aggregates are a more sensitive marker of in vivo platelet activation than platelet surface P-selectin: studies in baboons, human coronary intervention, and human acute myocardial infarction. Circulation. 2001;104(13):1533-7.

11. Furman MI, Barnard MR, Krueger LA, Fox ML, Shilale EA, Lessard DM, et al. Circulating monocyte-platelet aggregates are an early marker of acute myocardial infarction. J Am Coll Cardiol. 2001;38(4):1002-6.

12. Furman MI, Benoit SE, Barnard MR, Valeri CR, Borbone ML, Becker RC, et al. Increased platelet reactivity and circulating monocyte-platelet aggregates in patients with stable coronary artery disease. J Am Coll Cardiol. 1998;31(2):352-8.

13. McCabe DJ, Harrison P, Mackie IJ, Sidhu PS, Purdy G, Lawrie AS, et al. Platelet degranulation and monocyte-platelet complex formation are increased in the acute and convalescent phases after ischaemic stroke or transient ischaemic attack. $\mathrm{Br}$ ] Haematol. 2004;125(6):777-87.

14. Ruggeri ZM. The role of von Willebrand factor in thrombus formation. Thromb Res. 2007;120 Suppl 1:S5-9.

15. Popovic M, Smiljanic K, Dobutovic B, Syrovets T, Simmet T, Isenovic ER. Thrombin and vascular inflammation. Mol Cell Biochem. 2012;359(1-2):301-13.

16. Maclay JD, McAllister DA, Johnston S, Raftis J, McGuinnes C, Deans A, et al. Increased platelet activation in patients with stable and acute exacerbation of COPD. Thorax. 2011;66(9):76974. 
17. Munoz-Esquerre M, Ferreiro JL, Huertas D, Marcano AL, Lopez-Sanchez M, Roura G, et al. Impact of acute exacerbations on platelet reactivity in chronic obstructive pulmonary disease patients. Int J Chron Obstruct Pulmon Dis. 2018;13:141-8.

18. Polatli M, Cakir A, Cildag O, Bolaman AZ, Yenisey C, Yenicerioglu Y. Microalbuminuria, von Willebrand factor and fibrinogen levels as markers of the severity in COPD exacerbation. J Thromb Thrombolysis. 2008;26(2):97-102.

19. van der Vorm LN, Li L, Huskens D, Chayoua W, Kelchtermans H, de Groot PG, et al. Analytical characterization and reference interval of an enzyme-linked immunosorbent assay for active von Willebrand factor. PLoS One. 2019;14(2):e0211961.

20. van Mourik JA, Boertjes R, Huisveld IA, Fijnvandraat K, Pajkrt D, van Genderen PJ, et al. von Willebrand factor propeptide in vascular disorders: $A$ tool to distinguish between acute and chronic endothelial cell perturbation. Blood. 1999;94(1):179-85.

21. Husebø GR, Gabazza E, D'Alessandro C, Aanerud M, Toda M, Grønseth R, et al. Coagulation markers in COPD. European Respiratory Journal. 2018;52(suppl 62):OA1937.

22. 2019 Global Strategy for the Diagnosis, Management, and Prevention of COPD. Global Initiative for Chronic Obstructive Lung Disease (GOLD) 2018 [Available from: https://goldcopd. org/.

23. Huskens D, Roest M, Remijn JA, Konings J, Kremers RM, Bloemen S, et al. Strenuous exercise induces a hyperreactive rebalanced haemostatic state that is more pronounced in men. Thromb Haemost. 2016;115(6):1109-19.

24. Bloemen S, Huskens D, Konings J, Kremers RM, Miszta A, de Laat B, et al. Interindividual Variability and Normal Ranges of Whole Blood and Plasma Thrombin Generation. JALM. 2017;2(2):150-64.

25. Reilev M, Pottegard A, Lykkegaard J, Sondergaard J, Ingebrigtsen TS, Hallas J. Increased risk of major adverse cardiac events following the onset of acute exacerbations of COPD. Respirology. 2019;24(12):1183-90.

26. Wedzicha JA, Seemungal TA, MacCallum PK, Paul EA, Donaldson GC, Bhowmik A, et al. Acute exacerbations of chronic obstructive pulmonary disease are accompanied by elevations of plasma fibrinogen and serum IL-6 levels. Thromb Haemost. 2000;84(2):210-5.

27. Aleva FE, Temba G, de Mast O, Simons SO, de Groot PG, Heijdra YF, et al. Increased PlateletMonocyte Interaction in Stable COPD in the Absence of Platelet Hyper-Reactivity. Respiration. 2018;95(1):35-43.

28. Undas A, Jankowski M, Kaczmarek P, Sladek K, Brummel-Ziedins K. Thrombin generation in chronic obstructive pulmonary disease: dependence on plasma factor composition. Thromb Res. 2011;128(4):e24-8.

29. Wang M, Aaron CP, Madrigano J, Hoffman EA, Angelini E, Yang J, et al. Association Between Long-term Exposure to Ambient Air Pollution and Change in Quantitatively Assessed Emphysema and Lung Function. JAMA. 2019;322(6):546-56.

30. Aaron CP, Schwartz JE, Hoffman EA, Angelini E, Austin JHM, Cushman M, et al. A Longitudinal Cohort Study of Aspirin Use and Progression of Emphysema-like Lung Characteristics on CT Imaging: The MESA Lung Study. Chest. 2018;154(1):41-50.

31. Fawzy A, Putcha N, Aaron CP, Bowler RP, Comellas AP, Cooper CB, et al. Aspirin Use and Respiratory Morbidity in COPD: A Propensity Score-Matched Analysis in Subpopulations and Intermediate Outcome Measures in COPD Study. Chest. 2019;155(3):519-27. 
32. Wells JM, Colangelo LA, Sivarajan L, Thyagarajan B, Dransfield MT, Iribarren C, et al. Inflammation and endothelial activation in early adulthood are associated with future emphysema: the CARDIA Lung Study. Eur Respir J. 2019;53(1).

33. Lutsey PL, Folsom AR, Heckbert SR, Cushman M. Peak thrombin generation and subsequent venous thromboembolism: the Longitudinal Investigation of Thromboembolism Etiology (LITE) study. J Thromb Haemost. 2009;7(10):1639-48.

34. Amrani DL. Regulation of fibrinogen biosynthesis: glucocorticoid and interleukin- 6 control. Blood Coagul Fibrinolysis. 1990;1(4-5):443-6.

35. Stirling D, Hannant WA, Ludlam CA. Transcriptional activation of the factor VIII gene in liver cell lines by interleukin-6. Thromb Haemost. 1998;79(1):74-8.

36. Dielis AW, Castoldi E, Spronk HM, van Oerle R, Hamulyak K, Ten Cate H, et al. Coagulation factors and the protein $C$ system as determinants of thrombin generation in a normal population. J Thromb Haemost. 2008;6(1):125-31.

37. Altieri DC, Morrissey JH, Edgington TS. Adhesive receptor Mac-1 coordinates the activation of factor $X$ on stimulated cells of monocytic and myeloid differentiation: an alternative initiation of the coagulation protease cascade. Proc Natl Acad Sci U S A. 1988;85(20):7462-6.

38. Franco RF, de Jonge E, Dekkers PE, Timmerman JJ, Spek CA, van Deventer SJ, et al. The in vivo kinetics of tissue factor messenger RNA expression during human endotoxemia: relationship with activation of coagulation. Blood. 2000;96(2):554-9.

39. Cowan LT, Lutsey PL, Pankow JS, Matsushita K, Ishigami J, Lakshminarayan K. Inpatient and Outpatient Infection as a Trigger of Cardiovascular Disease: The ARIC Study. J Am Heart Assoc. 2018;7(22):e009683. 


\section{SUPPLEMENTAL DATA}

\section{Supplemental Methods}

Complete blood count and global clotting assays

Blood cell counts were measured with a Cell Dyn Sapphire analyser (Abbott Diagnostics, Santa Clara, CA). Prothrombin time (PT), activated partial thromboplastin time (aPTT) and D-dimer were measured in PPP on a STA-R Max analyser (Diagnostica Stago, Leiden, the Netherlands).

\section{Von Willebrand Factor parameters}

VWF antigen (VWF:Ag) was measured on a STA-R Max coagulation analyser (Stago, Leiden, the Netherlands). VWF ristocetin cofactor activity (VWF:RCo) was determined using the HemosIL@ AcuStar chemiluminescence assay (IL-Werfen, Bedford, MA, USA). VWF propeptide (VWFpp) was measured with an ELISA (Sanquin, Amsterdam, The Netherlands). ${ }^{1}$ Active VWF was quantified using an in-house ELISA assay, described previously. ${ }^{2}$ Results of the VWFpp and active VWF assay were normalized (\%) to normal pooled plasma (NPP) on the same plate. 


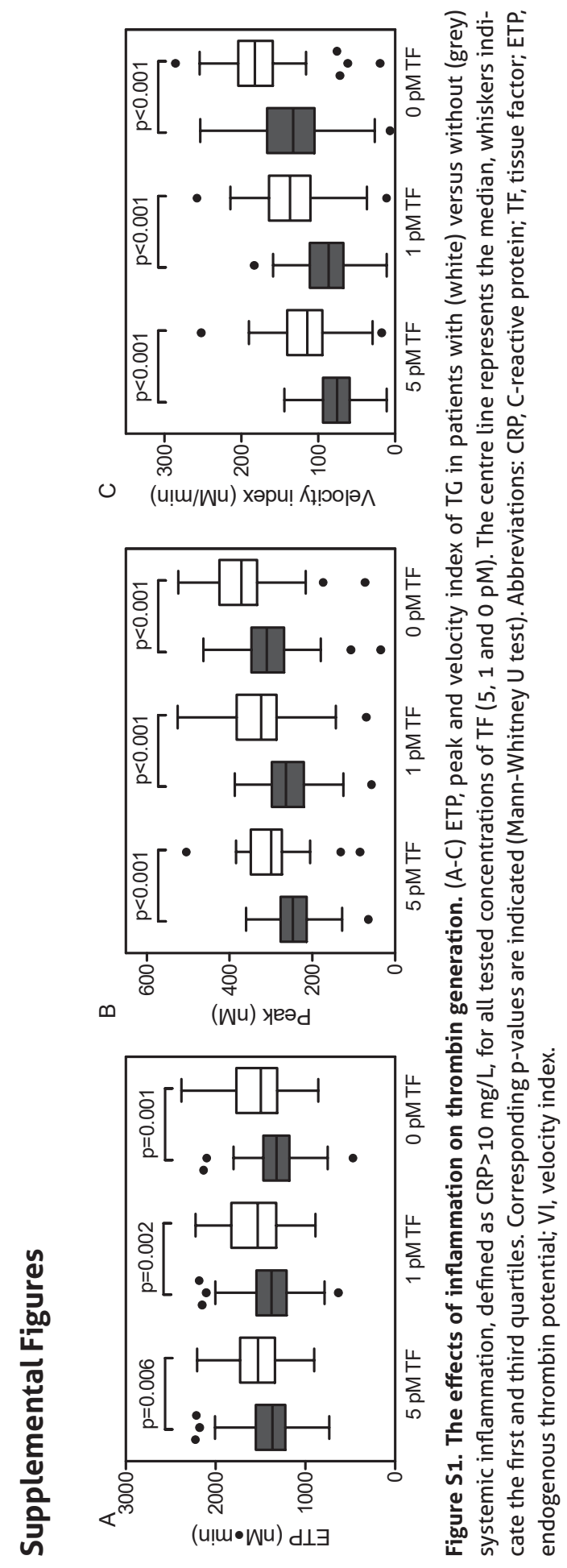




\section{Supplemental Tables}

Table S1. Complete blood count parameters during and after AE-COPD.

\begin{tabular}{|c|c|c|c|c|c|}
\hline Parameter & AE-COPD & Day 3 of treatment & Convalescence & p-value & $\begin{array}{l}\text { Reference } \\
\text { values }\end{array}$ \\
\hline Leukocytes, $\times 10^{9} / \mathrm{L}$ & $11.9 \pm 3.5^{*}$ & $12.2 \pm 3.5 \dagger$ & $10.0 \pm 3.4 * \dagger$ & $\begin{array}{l}* 0.015 \\
\dagger 0.003\end{array}$ & 4-11 \\
\hline Neutrophils, $\times 10^{9} / \mathrm{L}$ & $10.2 \pm 3.1^{*}$ & $8.8 \pm 3.4$ & $7.4 \pm 3.1^{*}$ & $* 0.001$ & $2-7.2$ \\
\hline Lymphocytes, $x 10^{9} / \mathrm{L}$ & $0.8(0.5) * \dagger$ & $2.4(1.4) * \S$ & $1.7(1.0) \dagger \S$ & $\begin{array}{l}*<0.001 \\
t<0.001 \\
\S 0.01\end{array}$ & $1-4$ \\
\hline Monocytes, $x 10^{9} / \mathrm{L}$ & $0.6 \pm 0.3$ & $1.0 \pm 0.3 * \dagger$ & $0.7 \pm 0.3 \dagger$ & $\begin{array}{l}*<0.001 \\
t<0.001\end{array}$ & $0.15-0.9$ \\
\hline Eosinophils, $x 10^{9} / \mathrm{L}$ & $0.02(0.03) *$ & $0.04(0.08) \dagger$ & $0.13(0.16) * \dagger$ & $\begin{array}{l}*<0.001 \\
t<0.001\end{array}$ & $0-0.5$ \\
\hline Basophils, $x 10^{9} / \mathrm{L}$ & $0.03(0.03) *$ & $0.04(0.06)$ & $0.06(0.06) *$ & $* 0.001$ & $0-0.15$ \\
\hline Erythrocytes, $x 10^{12} / L$ & $4.5 \pm 0.4 *$ & $4.4 \pm 0.4 * \dagger$ & $4.6 \pm 0.4 \dagger$ & $\begin{array}{r}* 0.005 \\
+0.001\end{array}$ & $3.8-6.0$ \\
\hline Platelets, $x 10^{9} / \mathrm{L}$ & 279 (108.5) & $281(132)$ & $272(120)$ & ns & $150-400$ \\
\hline$M P V, f L$ & $8.9 \pm 1.7^{*}$ & $8.9 \pm 1.8 \dagger$ & $8.2 \pm 1.3 * \dagger$ & $\begin{array}{l}t<0.001 \\
t<0.001\end{array}$ & $7.5-11.5$ \\
\hline
\end{tabular}

Data are presented as the mean \pm SD or median (IQR) for normally distributed and skewed continuous variables, respectively. $*, \dagger$ and $\S$ indicate the values that are significantly different from each other. Abbreviations: ns, non-significant; MPV, mean platelet volume. 
Table S2. Global coagulation assays and coagulation factors

\begin{tabular}{|c|c|c|c|c|c|}
\hline Parameter & AE-COPD & $\begin{array}{l}\text { Day } 3 \text { of } \\
\text { treatment }\end{array}$ & Convalescence & p-value & $\begin{array}{l}\text { Reference } \\
\text { values }\end{array}$ \\
\hline \multicolumn{6}{|c|}{ Global coagulation assays } \\
\hline PT, $s$ & $13.3(1.13)$ & $12.8(1.9)$ & $13.1(0.9)$ & ns & $11-14$ \\
\hline aPTT, s & $28(4.3)$ & $28(4.3)$ & $29(7.0)$ & ns & $30-40$ \\
\hline \multicolumn{6}{|c|}{ Markers of inflammation/acute phase proteins } \\
\hline CRP, $m g / L$ & $40.6 \pm 64.8 * 0$ & $20.8 \pm 26.9 * \dagger$ & $6.6 \pm 12.6 \dagger^{\circ}$ & $\begin{array}{l}*<0.001 \\
+0.005 \\
{ }^{\circ}<0.001\end{array}$ & $<10$ \\
\hline Fibrinogen, $g / L$ & $4.8 \pm 1.5^{*}$ & $4.2 \pm 1.3^{*}$ & $4.2 \pm 1.0$ & $* 0.001$ & $2-4$ \\
\hline \multicolumn{6}{|c|}{ Markers of in vivo coagulation } \\
\hline D-dimer, $n g / m L$ & $520(367)$ & $530(503)$ & $510(360)$ & ns & $<500$ \\
\hline \multicolumn{6}{|l|}{ VWF and FVIII } \\
\hline VWF:Ag, \% & $218(113)^{*}$ & $217(126) \dagger$ & $182(110) * \dagger$ & $\begin{array}{l}* 0.009 \\
t<0.001\end{array}$ & $50-150$ \\
\hline VWF:RCo, \% & $156.7(88.8)$ & $158.3(64.4) * \dagger$ & $135.1(71.4) * \dagger$ & $\begin{array}{l}* 0.009 \\
\dagger 0.002\end{array}$ & $50-150$ \\
\hline Active VWF, \% of NPP & $151(48) * 0$ & $142(32) * \dagger$ & $125(27) t^{\circ}$ & $\begin{array}{l}*<0.001 \\
t<0.001 \\
{ }^{\circ}<0.001\end{array}$ & $92-155$ \\
\hline VWFpp, \% of NPP & $123 \pm 48$ & $118 \pm 38$ & $109 \pm 42$ & ns & $73-189$ \\
\hline FVIII:C, \% & $185(91)^{*}$ & $198(87)^{* *}$ & $153(84)$ & $\begin{array}{l}* 0.001 \\
t<0.001\end{array}$ & $50-150$ \\
\hline
\end{tabular}

Data are presented as the mean \pm SD or median (IQR) for normally distributed and skewed continuous variables, respectively. *, $\dagger$ and $\S$ indicate the values that are significantly different from each other. Abbreviations: ns, non-significant; MPV, mean platelet volume. 
Table S3. Correlations between TG parameters and CRP.

\begin{tabular}{|c|c|c|}
\hline & \multicolumn{2}{|c|}{ Inflammation, CRP } \\
\hline & Spearman $r$ & $p$-value \\
\hline \multicolumn{3}{|l|}{5 pM TF } \\
\hline LT, $s$ & 0.339 & $<0.001$ \\
\hline ETP, nM.min & 0.244 & 0.008 \\
\hline Peak, $n M$ & 0.405 & $<0.001$ \\
\hline TTP, $s$ & -0.042 & ns \\
\hline $\mathrm{VI}, n M / \min$ & 0.450 & $<0.001$ \\
\hline \multicolumn{3}{|l|}{1 pM TF } \\
\hline LT, $s$ & 0.158 & ns \\
\hline ETP, nM.min & 0.277 & 0.002 \\
\hline Peak, $n M$ & 0.388 & $<0.001$ \\
\hline TTP, $s$ & -0.016 & ns \\
\hline VI, $n M / \min$ & 0.405 & $<0.001$ \\
\hline \multicolumn{3}{|l|}{0 pM TF } \\
\hline LT, $s$ & -0.009 & ns \\
\hline ETP, nM.min & 0.315 & 0.001 \\
\hline Peak, nM & 0.361 & $<0.001$ \\
\hline TTP, $s$ & -0.041 & ns \\
\hline VI, $n M / \min$ & 0.367 & $<0.001$ \\
\hline
\end{tabular}

Abbreviations: TF, tissue factor; LT, lagtime; ETP, endogenous thrombin potential; TTP, time to thrombin peak; VI, velocity index; ns, non-significant at the $p=0.05$ level 


\section{REFERENCES}

1. van Mourik JA, Boertjes R, Huisveld IA, Fijnvandraat K, Pajkrt D, van Genderen PJ, et al. von Willebrand factor propeptide in vascular disorders: $A$ tool to distinguish between acute and chronic endothelial cell perturbation. Blood. 1999;94(1):179-85.

2. van der Vorm LN, Li L, Huskens D, Chayoua W, Kelchtermans H, de Groot PG, et al. Analytical characterization and reference interval of an enzyme-linked immunosorbent assay for active von Willebrand factor. PLoS One. 2019;14(2):e0211961. 


$$
\text { Chapter } 7
$$




\section{Circulating active von Willebrand Factor levels are increased in chronic kidney disease and end-stage renal disease}

Van der Vorm LN, Visser R, Huskens D, Veninga A, Adams DL, Remijn JA, Hemker $H C$, Rensma PL, van Horssen $R$, de Laat $B$ 



\section{Dear Editors,}

Coagulation disorders are a common complication in patients with chronic kidney disease (CKD), with thrombosis being the most common cause of death. ${ }^{1}$ Recently Huang et al. ${ }^{2}$ found that patients with CKD are characterized by increased factor VIII (FVIII) activity and endothelial dysfunction, as apparent from increased von Willebrand factor (VWF) antigen levels.

VWF exerts a procoagulant effect by carrying FVIII and mediating platelet adhesion and aggregation. Under physiological conditions, VWF adopts a globular conformation and is unable to interact spontaneously with platelets. Pathologically, high shear or binding to exposed subendothelial collagen at sites of vascular injury can result in unfolding, thereby exposing the VWF A1 domain. ${ }^{3}$ This active conformation allows binding of VWF to the platelet glycoprotein Ib-IX-V receptor complex. ${ }^{4}$ Additionally, ultra large (UL)-VWF multimers are released from endothelial cells upon activation. These UL-VWF multimers are in their active, platelet-binding conformation, but are normally cleaved immediately by a disintegrin and metalloproteinase with thrombospondin type 1 motif, member 13 (ADAMTS13). ${ }^{4}$ Circulating active VWF (i.e. UL-VWF or VWF exposing the A1 domain due to high shear stress) was previously demonstrated to be increased in various patient populations suffering from thrombotic complications. ${ }^{5}$ Therefore, in this pilot study we assessed if levels of active VWF are also affected by various stages of kidney disease.

Using an enzyme-linked immunosorbent assay $^{6}$, we determined active VWF levels in plasma from a cohort of patients (55\% male) with renal disease, recruited in the Elisabeth-TweeSteden Hospital in Tilburg, the Netherlands. The cohort comprised patients not receiving haemodialysis (CKD, $n=25$, mean age $61 \pm 13$ years, body mass index [BMI] $29.3 \pm 6.6 \mathrm{~kg} / \mathrm{m}^{2}$, estimated glomerular filtration rate [eGFR] $29 \pm 14 \mathrm{~mL} / \mathrm{min} / 1.73$ $\left.\mathrm{m}^{2}\right)$, patients with end-stage renal disease (ESRD) undergoing thrice weekly haemodialysis (HD-ESRD, $\mathrm{n}=13$, mean age $65 \pm 15$ years, $\mathrm{BMI} 26.6 \pm 4.8 \mathrm{~kg} / \mathrm{m}^{2}$ ) and patients with ESRD on peritoneal dialysis (PD-ESRD, $n=4$, mean age $62 \pm 20$ years, BMI $26.1 \pm 4.3 \mathrm{~kg}$ / $\mathrm{m}^{2}$ ). Macro-albuminuria (albumin-to-creatinine ratio [ACR]: $\mathrm{men}>25 \mathrm{mg} / \mathrm{mmol}$, women $>35 \mathrm{mg} / \mathrm{mmol}$ ) was unknown for HD-ESRD patients, but was present in 17 (68\%) CKD patients and 3 (75\%) PD-ESRD patients.

A history of thrombosis was present in three (12\%) CKD patients (one deep vein thrombosis, two ischaemic stroke), two (15\%) HD-ESRD patients (one myocardial infarction, one ischaemic stroke) and one (25\%) PD-ESRD patient (deep vein thrombosis). A group of healthy volunteers ( $n=31,36 \%$ male, mean age $50 \pm 12$ years, $B M I 23.3 \pm 2.5$, eGFR $>60$ $\mathrm{mL} / \mathrm{min} / 1.73 \mathrm{~m}^{2}$, no proteinuria and no history of thrombosis) served as unmatched 
controls. All participants gave full informed consent according to the 2013 Declaration of Helsinki and the study was approved by the Medical Ethical Committee of the Elisabeth-TweeSteden Hospital, Tilburg, The Netherlands (study NL45975.008.14).

In the circulation of all patient groups, active VWF levels were elevated compared with healthy controls (median [01-03] 107.6\% [86.4-132.8]) (Figure 1A). Although the differences between CKD (135.8\% [115.8-179.6]), HD-ESRD (158.1\% [122.7-198.4]) and PD-ESRD (233.3\% [195.8-275.8]) patients were not significant, a trend towards increased active VWF with increased disease severity was observed. Of note, plasma levels of total VWF were also considerably higher in CKD (153.6\% [141.7-171.3]), HDESRD (149.7\% [130.2-163.2]) and PD-ESRD (152.0\% [142.6-162.4]) patients than in healthy controls (108.2\% [90.0-130.0]) (Figure 1B), corroborating previous reports ${ }^{2,7}$, but did not show the same trend with increased disease severity.

A

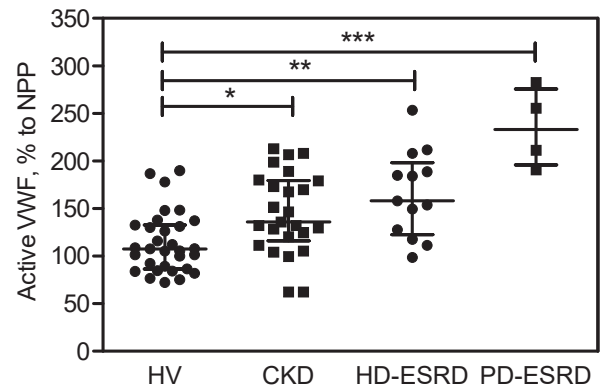

B

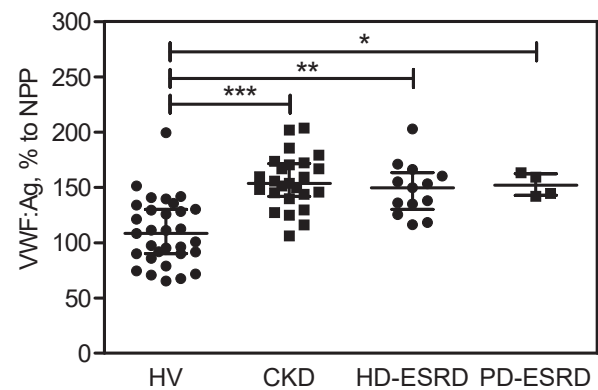

Figure 1. Active VWF and total VWF are elevated in kidney disease compared with healthy controls. Levels of (A) active VWF and (B) total VWF, both normalized to levels in normal pooled plasma, were measured in patients with CKD, HD-ESRD or PD-ESRD and healthy volunteers. Bars represent the median and whiskers represent the first and third quartiles. P-values $<0.05$ were considered significant using Kruskal-Wallis test with post hoc Dunn's multiple comparison test. $* p<0.05, * p<0.01, \cdots p<0.001$. All statistical analyses were performed using Graphpad Prism version 7 (GraphPad Software, San Diego, CA, USA).

The underlying pathophysiological mechanism is likely related to chronic exposure of endothelium to inflammatory cytokines and oxidative stress. ${ }^{8}$ As a result, with progressing stages of kidney disease, ongoing damage and/or activation of the endothelium lead to the release of increasingly large amounts of UL-VWF into the circulation. The degree of endothelial activation may also be related to the dialysis modality, as a trend towards higher active VWF levels in PD-ESRD compared with HD-ESRD patients can be observed. Additionally, a previous study ${ }^{9}$ found substantially decreased ADAMTS13 levels in CKD patients compared with controls. This reduction in ADAMTS13 levels may be caused by increased loss via urine due to damage to the glomerular filtration mem- 
brane. Consequently, a proportion of UL-VWF will not be proteolyzed, but remains in its active conformation. Interestingly, we observed a significant correlation (Spearman $r=0.390, p=0.0021$ ) between urinary protein (including ADAMTS13) loss (measured as ACR) and circulating active VWF levels in our study population.

In summary, circulating levels of total VWF and, even more pronounced, active VWF are increased in patients with CKD and ESRD compared with healthy controls. High plasma VWF levels were previously found to be a predictor of microalbuminuria, CVD and mortality in both diabetic and non-diabetic patients. ${ }^{10}$ We hypothesize that active VWF, being the haemostatically active fraction of VWF, may be even more strongly associated with cardiovascular morbidity and mortality. However, follow-up studies with larger patient numbers are required to determine the mechanism behind increased active VWF in CKD, by examining the association between active VWF, circulating and urinary ADAMTS13 levels and the presence of an arteriovenous fistula. In addition, clinical follow-up data are required to unravel the relationship of active VWF and clinical outcome.

\section{ACKNOWLEDGEMENTS}

We thank V.J.F. Strijbis and R.M.W. Kremers for their assistance with the plasma samples. 


\section{REFERENCES}

1. Wattanakit K, Cushman M, Stehman-Breen C, Heckbert SR, Folsom AR. Chronic kidney disease increases risk for venous thromboembolism. J Am Soc Nephrol. 2008;19(1):135-40.

2. Huang MJ, Wei RB, Wang Y, Su TY, Di P, Li OP, et al. Blood coagulation system in patients with chronic kidney disease: a prospective observational study. BMJ Open. 2017;7(5):e014294.

3. Huizinga EG, Tsuji S, Romijn RA, Schiphorst ME, de Groot PG, Sixma Jj, et at. Structures of glycoprotein Ibalpha and its complex with von Willebrand factor A1 domain. Science. 2002;297(5584):1176-9.

4. Ruggeri ZM. The role of von Willebrand factor in thrombus formation. Thromb Res. 2007;120 Suppl 1:S5-9.

5. Groot E, de Groot PG, Fijnheer R, Lenting PJ. The presence of active von Willebrand factor under various pathological conditions. Curr Opin Hematol. 2007;14(3):284-9.

6. van der Vorm LN, Li L, Huskens D, Chayoua W, Kelchtermans H, de Groot PG, et al. Analytical characterization and reference interval of an enzyme-linked immunosorbent assay for active von Willebrand factor. PLoS One. 2019;14(2):e0211961.

7. Pequeriaux NC, Fijnheer R, Gemen EF, Barendrecht AD, Dekker FW, Krediet RT, et al. Plasma concentration of von Willebrand factor predicts mortality in patients on chronic renal replacement therapy. Nephrol Dial Transplant. 2012;27(6):2452-7.

8. Merino A, Nogueras S, Buendia P, Ojeda R, Carracedo J, Ramirez-Chamond R, et al. Microinflammation and endothelial damage in hemodialysis. Contrib Nephrol. 2008;161:83-8.

9. Shen L, Lu G, Dong N, Jiang L, Ma Z, Ruan C. Von Willebrand factor, ADAMTS13 activity, TNF-alpha and their relationships in patients with chronic kidney disease. Exp Ther Med. 2012;3(3):530-4.

10. Jager A, van Hinsbergh VW, Kostense PJ, Emeis J], Yudkin JS, Nijpels G, et al. von Willebrand factor, C-reactive protein, and 5-year mortality in diabetic and nondiabetic subjects: the Hoorn Study. Arterioscler Thromb Vasc Biol. 1999;19(12):3071-8. 



$$
\text { Chapter } 8
$$




\title{
Active von Willebrand Factor in patients with a bleeding diathesis
}

\author{
Van der Vorm LN, Huskens D, Florin L, De \\ Kesel P, Roest M, de Laat B, Devreese KMJ
}



Dear Editors,

Whereas the diagnosis of established bleeding disorders, such as haemophilia, is well defined, there are many patients with a mild to moderate bleeding diathesis (e.g. frequent epistaxis, menorrhagia, prolonged post-operative bleeding) of unknown cause, who pose a significant diagnostic challenge. ${ }^{1}$ Following initial screening based on personal/family history, bleeding scores, full blood cell count and routine coagulation screening tests (e.g. PT and aPTT), these patients can be referred for further evaluation of clotting factor activity, platelet function testing (e.g. PFA-200 ${ }^{\circledR}$, light transmission aggregometry (LTA)) and von Willebrand factor (VWF) antigen/activity. ${ }^{2}$ The diagnosis of von Willebrand Disease (VWD) is based on VWF antigen concentration (VWF:Ag) and VWF function tests, most commonly the VWF ristocetin cofactor activity (VWF:RCo). ${ }^{3}$ However, VWF:RCo assays suffer from high variability and may give falsely low VWF activities as a result of polymorphisms. A more recently developed assay uses recombinant GPIb fragments with two gain-of-function mutations that allow binding to VWF (VWF:GPIbM). ${ }^{3}$ Although this assay does not require ristocetin like the VWF:RCo assay, it is based on non-physiological binding of VWF to a mutant receptor.

Recently, we developed an immunosorbent assay, based on a variable heavy chain antibody fragment (VHH), to quantify active VWF. This is the haemostatically active fraction of VWF that is circulating in vivo in its platelet GP1b $\alpha$-binding conformation. In a healthy study population, only a small proportion of VWF was in its active conformation. ${ }^{4}$ Several pathological conditions have been associated with increased levels of active VWF, using an assay based on the same VHH for active VWF. ${ }^{5}$ The spontaneous interaction between active VWF and platelets can tilt the haemostatic balance to either the prothrombotic (e.g. TTP and HELLP syndrome, associated with on average 2 to 12fold and 3-fold increased active VWF levels compared to healthy controls, respectively) or the bleeding (e.g. von Willebrand disease (VWD) type 2B) side..$^{5}$ In VWD type 2B, enhanced binding of platelets to active VWF (2-15 fold increased levels compared to healthy controls ${ }^{5}$, due to gain-of-function mutations in the A1 domain) results in clearance of high-molecular-weight (HMW) VWF multimers and platelets, and consequently a bleeding phenotype.

However, it is unclear whether the proportion of activated VWF in the circulation also plays a role in patients with mild to moderate bleeding without thrombocytopenia. It can be hypothesized that, since individuals with relatively high circulating active VWF levels are more at risk for spontaneous platelet-aggregate formation, relatively low circulating active VWF levels may delay clot formation and hence contribute to a mild bleeding diathesis. Therefore, we investigated whether in a population of patients with 
a mild bleeding diathesis there were more patients with very low active VWF levels compared to a control population. In addition, we sought to identify cases in which active VWF levels may have additional diagnostic value over the commonly used VWF:Ag and VWF:RCo assays.

Between December 2014 and December 2017, patients with a mild bleeding diathesis of unknown cause were recruited for participation in the current study in the Ghent University Hospital in Belgium. All patients were referred by their haematologist for laboratory evaluation for diagnosis of a bleeding disorder. The types of bleeding seen in these patients were frequent hematomas $(n=23)$, epistaxis $(n=19)$, menorrhagia $(n=6)$, petechiae/ecchymoses $(n=9)$, heavy postpartum bleeding $(n=6)$, prolonged postsurgical bleeding $(n=10)$, gingival bleeding $(n=4)$ and combinations of these bleeding symptoms. For 13 patients there was a clinical suspicion of von Willebrand's disease. The study was approved by the Ethical Committee of the Ghent University Hospital. Citrated whole blood was collected by antecubital venipuncture (BD Vacutainer, Becton Dickinson, Plymouth, UK). Platelet-poor plasma (PPP) was prepared and subsequently stored at $-80^{\circ} \mathrm{C}$ until further testing. Active VWF was quantified using an ELISA, based on a variable heavy chain antibody (VHH) specific for VWF in its GP1b $\alpha$-binding conformation, as described previously. ${ }^{4}$ Normal pooled plasma (NPP) was used as a standard in every plate and sample results were normalized (\%) to NPP on the same plate. VWF antigen (VWF:Ag) and VWF:RCo were measured using the HemosIL® AcuStar VWF:Ag assay and the HemosIL $®$ AcuStar VWF:RCo assay, respectively (IL-Werfen, Bedford, MA, USA). Platelet-VWF binding (Plt:VWF) was measured by flow cytometry, as described previously, ${ }^{4}$ and is expressed as median fluorescence intensity (MFI), corrected for the MFI of the control condition without ristocetin. The ISTH-BAT bleeding scores were determined by consulting the medical records, by one and the same person (clinical pathologist) to avoid bias, and were considered elevated if $>5$ in women, $>3$ in men and $>2$ in children. ${ }^{6}$ Associations between continuous variables were determined using the non-parametric Spearman's rank correlation coefficient. Differences in active VWF levels between two groups were compared using the non-parametric Mann-Whitney $\mathrm{U}$ test. Comparison of active VWF levels between three groups was performed using the non-parametric Kruskal-Wallis test. A p-value of 0.05 was considered statistically significant for all comparisons.

We included 145 patients (32 men, 89 women, 24 children) with a bleeding diathesis of unknown cause. The ISTH-BAT bleeding score was documented for 109 subjects (24 men, 68 women, 17 children), and was increased above the respective reference ranges ${ }^{6}$ for 10 men, 12 women and 7 of the included children. 
Active VWF levels in the entire study population $(n=145)$ correlated significantly with all other VWF parameters (except Plt:VWF binding with low ristocetin), with almost identical correlation coefficients to those previously found in a healthy population. ${ }^{4}$ The strongest correlation (Spearman's $r=0.442, p<0.001$ ) existed between active VWF and VWF:Ag, followed by VWF:RCo $(r=0.399, p<0.001)$ and Plt-VWF binding in the presence of a high $(1.2 \mathrm{mg} / \mathrm{mL})$ concentration of ristocetin $(r=0.386, p<0.001)$.

In these subjects with a bleeding tendency, active VWF, VWF:Ag levels and VWF:RCo activity were relatively lower, albeit not statistically significant, compared to the previously determined reference intervals ${ }^{7}$, especially the reference interval for blood group non-O individuals (Figure $1 A-D$ ). Several individuals had higher, but none had lower Plt:VWF binding in response to ristocetin compared to the reference range in healthy controls (Figure $1 \mathrm{E}, \mathrm{F}$ ). Red dots indicate individuals with a significant bleeding score. Active VWF was not significantly different for subjects with versus without an increased bleeding score (Figure 2A), although it must be noted that the bleeding score does not always correlate optimally with clinical bleeding tendency. ${ }^{8}$

Active VWF was not significantly different between subjects with suspected VWD versus other suspected causes of bleeding diathesis (Figure 2B). In VWD 2B patients, bleeding is due to thrombocytopenia as a result of the clearance of platelet-VWF-aggregates. In the current study 31 patients had a platelet count below $150 \times 10^{9} / \mathrm{L}$, of which three patients had a platelet count below $50 \times 10^{9} / \mathrm{L}$. Only one of these three thrombocytopenic patients had active VWF levels above the reference range (209.2\%), concurrently with mildly elevated VWF:Ag (241.6\%) and normal VWF:RCo activity (206.4\%), hence this thrombocytopenia is not due to VWD type $2 \mathrm{~B}$, but may rather be explained by a thrombocytopathy.

In one patient with a bleeding diathesis, active VWF levels were increased almost 5-fold (497\%), in conjunction with substantially increased VWF:Ag (323.5\%) and mildly elevated VWF:RCo (234.3\%) and FVIII (200.0\%) levels. The platelet count was normal $\left(232 \times 10^{9} / \mathrm{L}\right)$. However, light transmission aggregometry (LTA) demonstrated a platelet ATP secretion defect and primary wave disaggregation upon stimulation with epinephrine. A second patient with strongly elevated active VWF levels (247.5\%) had a similar pattern of increased VWF level and activity, and was also suspected for a platelet secretion defect. Thus, the bleeding symptoms in these patients can be explained by platelet secretion defects, but the cause of the high (active) VWF levels and activity in these patients remains unknown. However, since both were elderly patients (70 and 77 years) they may be (partly) attributed to comorbidities that affect endothelial VWF secretion and activation, in particular inflammatory conditions. Inflammation induces 


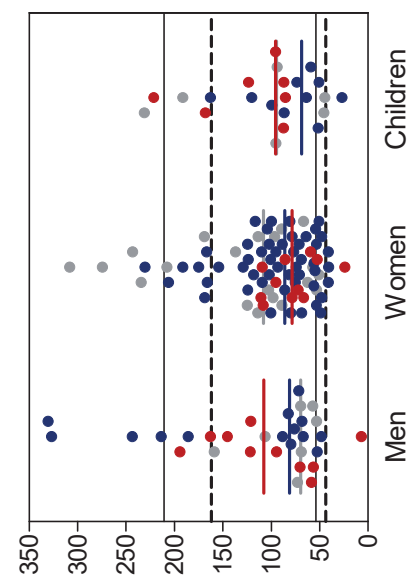

(\%) оэप: $\lrcorner M$

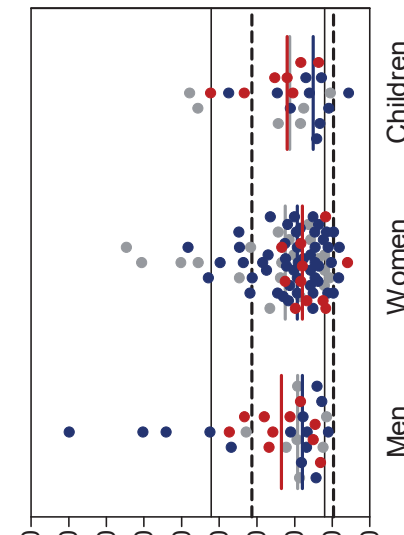

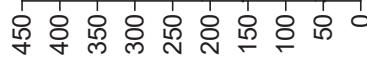

๓

(\%) $6 \forall: \exists M \wedge$

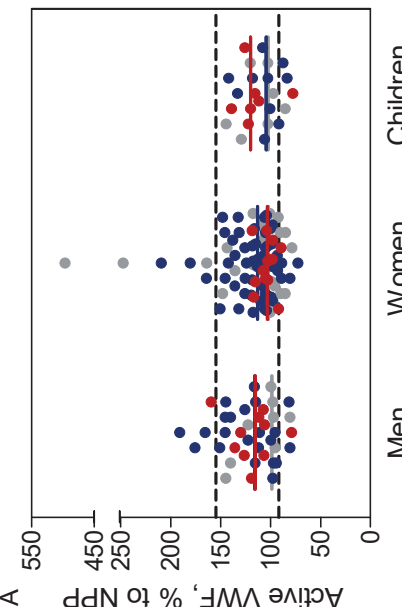

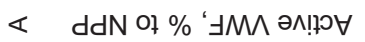

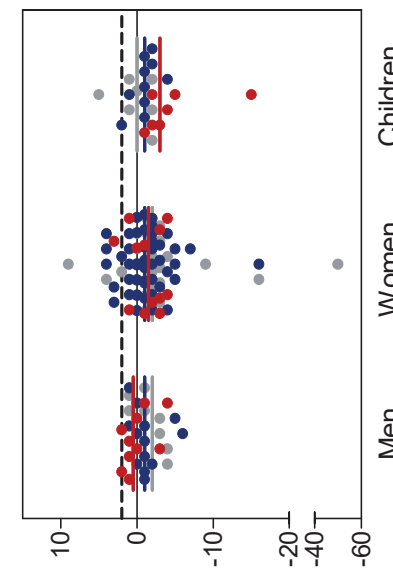

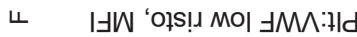

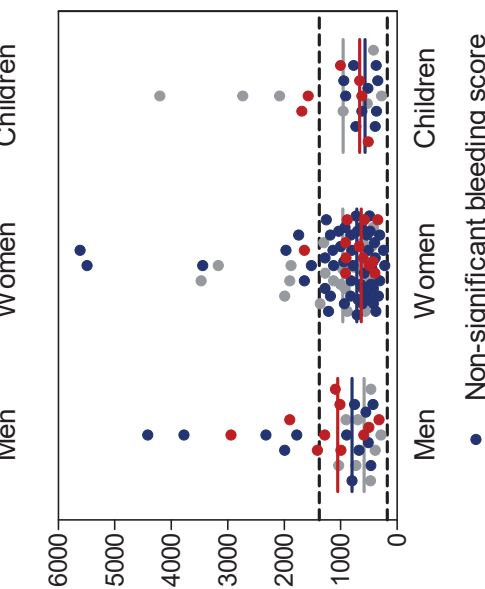

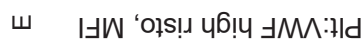

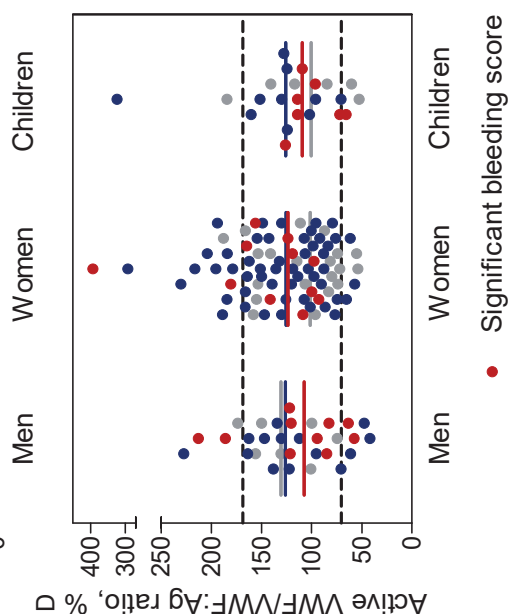

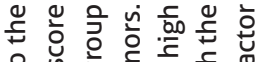

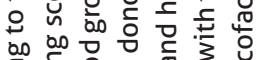

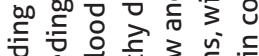

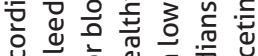
讨 훙

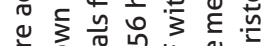

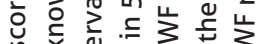

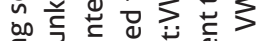
동 षै

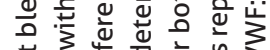

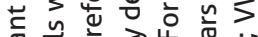

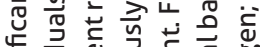

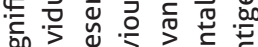

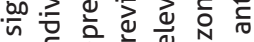

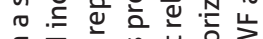

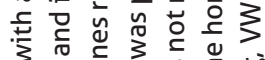
उ.

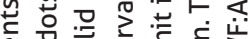
बั

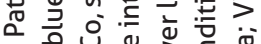
ย்

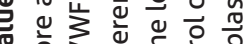
$>$ 언 \&

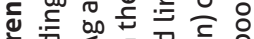

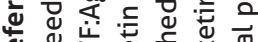

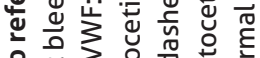

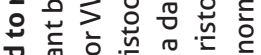

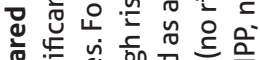

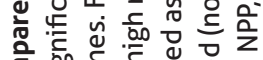
ह

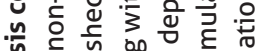

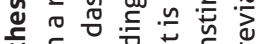

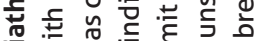

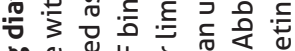

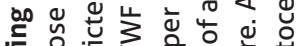

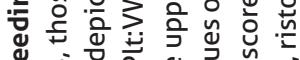

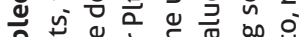

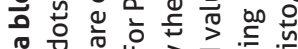
단

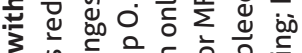
उद

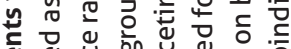
ब

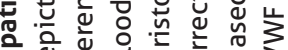

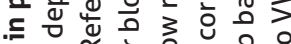

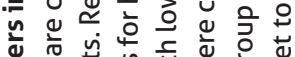

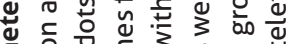
है을 일 论

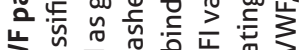

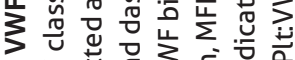
단.

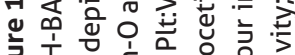

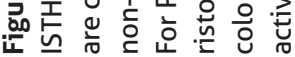



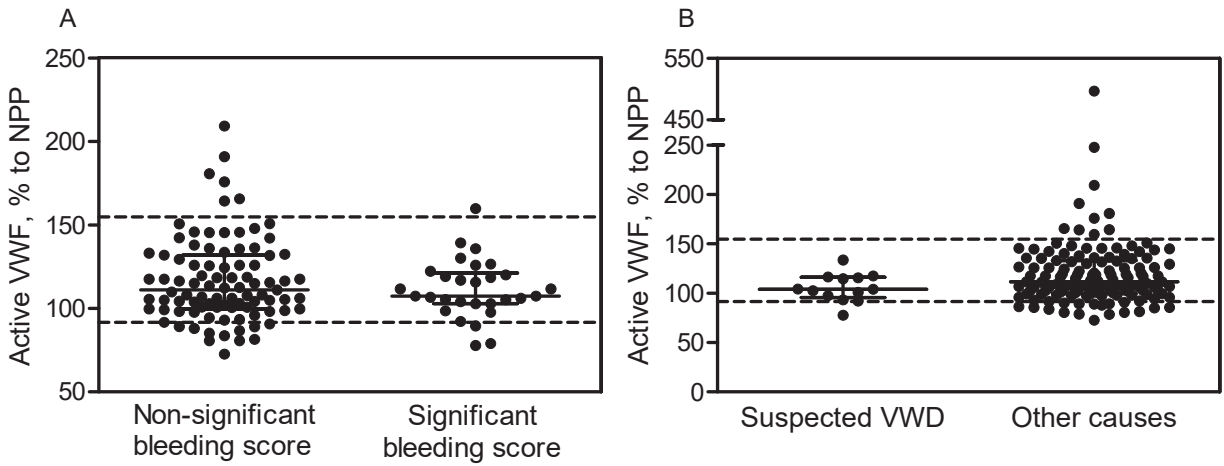

Figure 2. Active VWF related to clinically significant bleeding and suspected VWD. (A) Active VWF is not different in subjects with significant bleeding (defined as $>5$ in women, $>3$ in men and $>2$ in children) and (B) not different in subjects with suspected von Willebrand disease versus other suspected causes of the bleeding diathesis. Dashed lines delineate the reference interval determined in a previous study in healthy individuals. ${ }^{4}$ Horizontal bars represent the median. Abbreviations: NPP, normal pooled plasma; VWD, von Willebrand disease.

activation of the endothelium, resulting in excessive release of VWF into the circulation, whereas inflammation reduces the activity of the VWF-cleaving protease ADAMTS13, hence active VWF levels increase. ${ }^{9}$ Unfortunately, no inflammatory markers (e.g. CRP) were measured to confirm this was the cause of the elevated active VWF levels.

One patient with an active VWF level that was not above the reference interval but relatively high (142\%) compared to the low VWF:Ag (54.6\%) and VWF:RCo (50.8\%) was diagnosed with Henoch-Schönlein purpura. This is a rare inflammatory disease of the small blood vessels. The presence of abnormally large (and hence active) multimers has previously been described for patients with this disease. ${ }^{10}$

On the other end of the spectrum, three patients with a bleeding tendency of unknown cause had active VWF levels below the lower limit of the reference interval (91.6\%). All also had VWF:Ag (41.6-66.6\%) and VWF:RCo (41.2-57.9\%) on the lower end of their reference intervals with normal FVIII levels (69.1-86.2\%) and platelet count (159-269 $\left.\times 10^{9} / \mathrm{L}\right)$. Their bleeding symptoms were not more severe or distinct from those of the patients with increased active VWF, hence the decreased active VWF levels seem to have no clinical relevance in these patients.

In conclusion, in this highly heterogeneous population of individuals with a bleeding diathesis, active VWF levels were on average slightly, albeit not significantly, lower compared to healthy individuals. The diagnosis of mild bleeding disorders is very challenging, and in approximately half of the cases, the underlying cause is not found. ${ }^{1}$ This 
study adds that, in this population, quantification of active VWF levels does not have added diagnostic value to VWF:Ag and VWF activity assays in the diagnosis of unexplained bleeding disorders.

\section{ACINOWLEDGEMENTS}

We would like to thank Diëgo Arnoe, Julie De Leenheer, An De Saar, Michael Luypaert and Esha Wauters for their technical support. 


\section{REFERENCES}

1. Quiroga T, Mezzano D. Is my patient a bleeder? A diagnostic framework for mild bleeding disorders. Hematology Am Soc Hematol Educ Program. 2012;2012:466-74.

2. Hayward CPM. How I investigate for bleeding disorders. Int J Lab Hematol. 2018;40 Suppl 1:6-14.

3. Favaloro EJ. Diagnosis or Exclusion of von Willebrand Disease Using Laboratory Testing. Methods Mol Biol. 2017;1646:391-402.

4. van der Vorm LN, Li L, Huskens D, Chayoua W, Kelchtermans H, de Groot PG, et al. Analytical characterization and reference interval of an enzyme-linked immunosorbent assay for active von Willebrand factor. PLoS One. 2019;14(2):e0211961.

5. Groot E, de Groot PG, Fijnheer R, Lenting PJ. The presence of active von Willebrand factor under various pathological conditions. Curr Opin Hematol. 2007;14(3):284-9.

6. Rodeghiero F, Tosetto A, Abshire T, Arnold DM, Coller B, James P, et al. ISTH/SSC bleeding assessment tool: a standardized questionnaire and a proposal for a new bleeding score for inherited bleeding disorders. J Thromb Haemost. 2010;8(9):2063-5.

7. Verfaillie CJ, De Witte E, Devreese KM. Validation of a new panel of automated chemiluminescence assays for von Willebrand factor antigen and activity in the screening for von Willebrand disease. Int J Lab Hematol. 2013;35(5):555-65.

8. Fasulo MR, Biguzzi E, Abbattista M, Stufano F, Pagliari MT, Mancini I, et al. The ISTH Bleeding Assessment Tool and the risk of future bleeding. J Thromb Haemost. 2018;16(1):125-30.

9. Bernardo A, Ball C, Nolasco L, Moake JF, Dong JF. Effects of inflammatory cytokines on the release and cleavage of the endothelial cell-derived ultralarge von Willebrand factor multimers under flow. Blood. 2004;104(1):100-6.

10. Casonato A, Pontara E, Bertomoro A, Ossi E, Vincenti M, Girolami A, et al. Abnormally large von Willebrand factor multimers in Henoch-Schonlein purpura. Am J Hematol. 1996;51(1):7-11. 


$$
\text { Chapter } 9
$$




\title{
Effects of plasmin on von Willebrand Factor and platelets: a narrative review
}

\author{
Van der Vorm LN, Remijn JA, de Laat B,
} Huskens D 


\section{ABSTRACT}

Plasmin is the major fibrinolytic protease responsible for dissolving thrombi by cleavage of its primary substrate fibrin. In addition, emerging evidence points to other roles of plasmin: [1] as a back-up for ADAMTS13 in proteolysis of ultra large von Willebrand factor (VWF) multimers and [2] as an activator of platelets. Whereas the molecular mechanisms of fibrinolysis are well defined, insights on the effects of plasmin on VWF and platelets are relatively scarce and sometimes conflicting. Hence, this review provides an overview of the literature on the effects of plasmin on VWF multimeric structures, on VWF binding to platelets and on platelet activation. This information is placed in the context of possible applications of thrombolytic therapy for the condition thrombotic thrombocytopenic purpura. 


\section{INTRODUCTION}

Plasmin is the key protease of the fibrinolytic system. During clot development, fibrin deposition elicits generation of plasmin from plasminogen, resulting in the dissolution of the fibrin clot. ${ }^{1}$ Whereas many studies have focused on mechanisms of plasminmediated fibrin cleavage (reviewed by, amongst others, Cesarman-Maus ${ }^{2}$ ), there is a relative gap in knowledge on the effects of plasmin on other components that form the primary blood clot/thrombus. Recently there has been increasing attention for a potential role of plasmin in the treatment of disorders associated with spontaneous VWF-platelet aggregation, such as thrombotic thrombocytopenic purpura (TTP). ${ }^{3,4}$ This review provides an overview of the literature on the effects of plasmin on von Willebrand factor (VWF) and platelets. After a brief introduction into the structure and functions of plasmin, the evidence supporting a role for plasmin in degradation of VWF multimers and VWF-platelet complexes are presented. This is followed by a summary of existing literature on the effect of plasmin on platelet activation through the platelet GP1b, GPIIb/IIla and thrombin receptors.

\section{PLASMIN FUNCTION AND REGULATION}

Plasmin is a potent serine-protease that is generated from its zymogen, plasminogen, usually at sites of vessel damage. Synthesized primarily in the liver ${ }^{5}$, plasminogen circulates in plasma at a concentration of approximately $1.5 \mu \mathrm{mol} / \mathrm{l}$, with a half-life of about 2 days. ${ }^{6}$ Native, circulating Glu-plasminogen ( $92 \mathrm{kDa}$ ) is a single chain glycoprotein with a glutamic acid (Glu) as the $\mathrm{N}$-terminal residue, containing an $\mathrm{N}$-terminal activation peptide (NTP), five homologous triple-loop structures called kringle domains (K1-K5), and the serine protease domain containing the catalytic triad (Figure 1). ${ }^{7}$ Physiological activation of plasminogen is mediated by tissue-type plasminogen activator (tPA) and urokinase-type plasminogen activator (uPA, urokinase). ${ }^{6}$ tPA and uPA specifically cleave the single activation bond Arg561-Val562 of plasminogen, resulting in the formation of the two-chain enzyme plasmin composed of an N-terminal heavy chain (12-65 kDa) and a C-terminal light chain $(25 \mathrm{kDa}){ }^{8}$ Activation of plasminogen is inhibited by plasminogen activator inhibitors types 1 and 2 (PAI-1 and PAI-2). ${ }^{9,10}$

The primary substrate of plasmin is fibrin, which regulates its own degradation by binding both plasminoge ${ }^{12}$ and tPA $^{13}$ on its surface, thereby localizing and enhancing plasmin generation. This binding of plasminogen to fibrin can be blocked by thrombinactivatable fibrinolysis inhibitor (TAFI). ${ }^{14}$ Importantly, plasmin can also cleave both tPA and UPA, transforming them from single chain to more active two-chain polypeptides, 


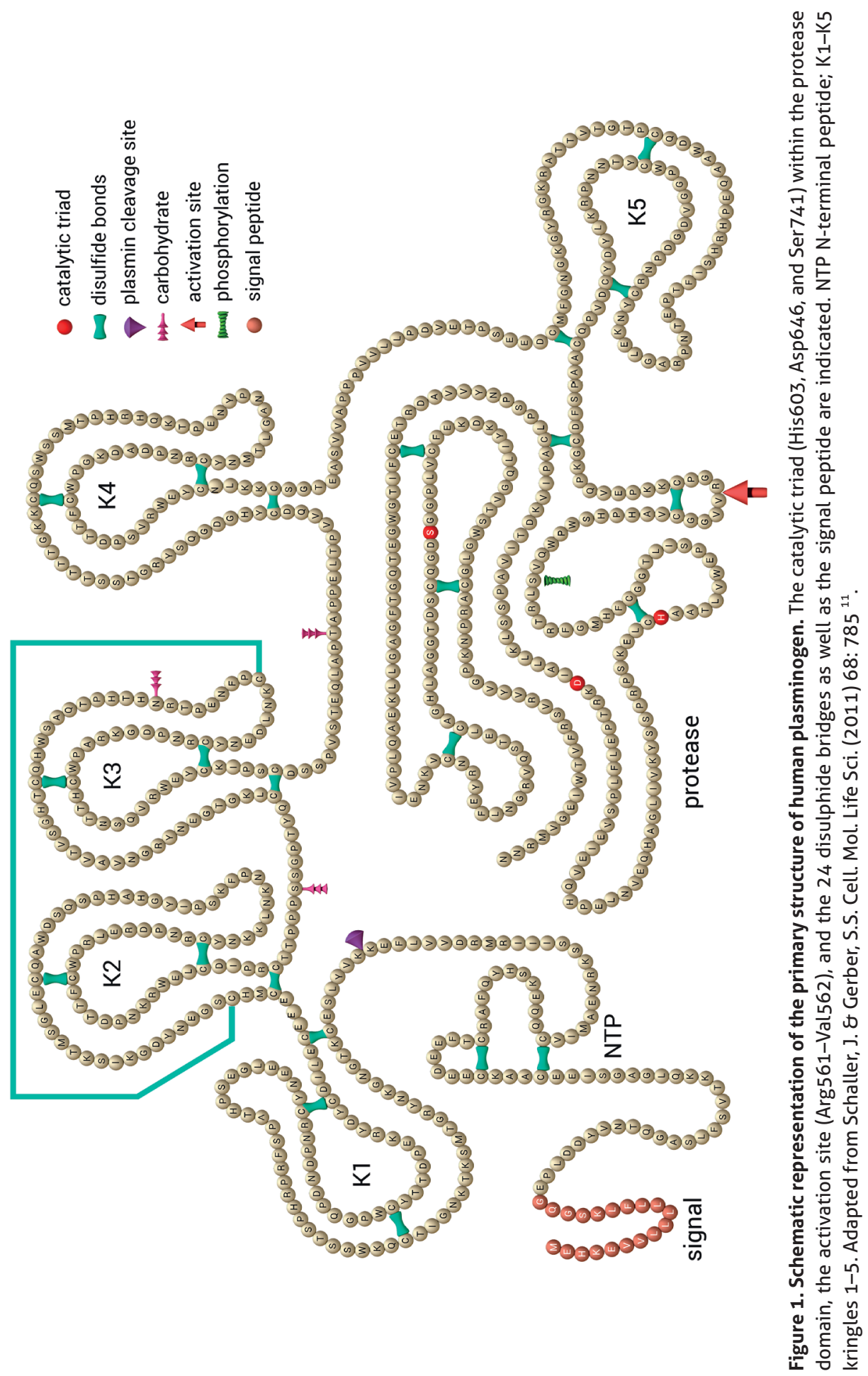


thereby forming a positive-feedback loop (Figure 2). ${ }^{2}$ Plasmin is rapidly inhibited by its major inhibitor $\alpha 2$-antiplasmin ( $\alpha 2$-AP), and to a lesser extent by $\alpha 2$-macroglobulin $(\alpha 2 \mathrm{M})$, unless it remains bound to fibrin or to its cell surface receptors (Figure 2$).{ }^{15}$

Plasmin does not only cleave fibrin, it possesses exceptionally broad specificity for target substrates, targeting amongst others coagulation factors $\mathrm{V}^{16}, \mathrm{VIII}^{17}, \mathrm{IX}^{18}$ and $\mathrm{X}^{19}$, $\mathrm{TFPI}^{20}$, fibrinogen ${ }^{21}$ as well as the VWF cleaving protease ADAMTS13 ${ }^{22}$ (Table 1 ).

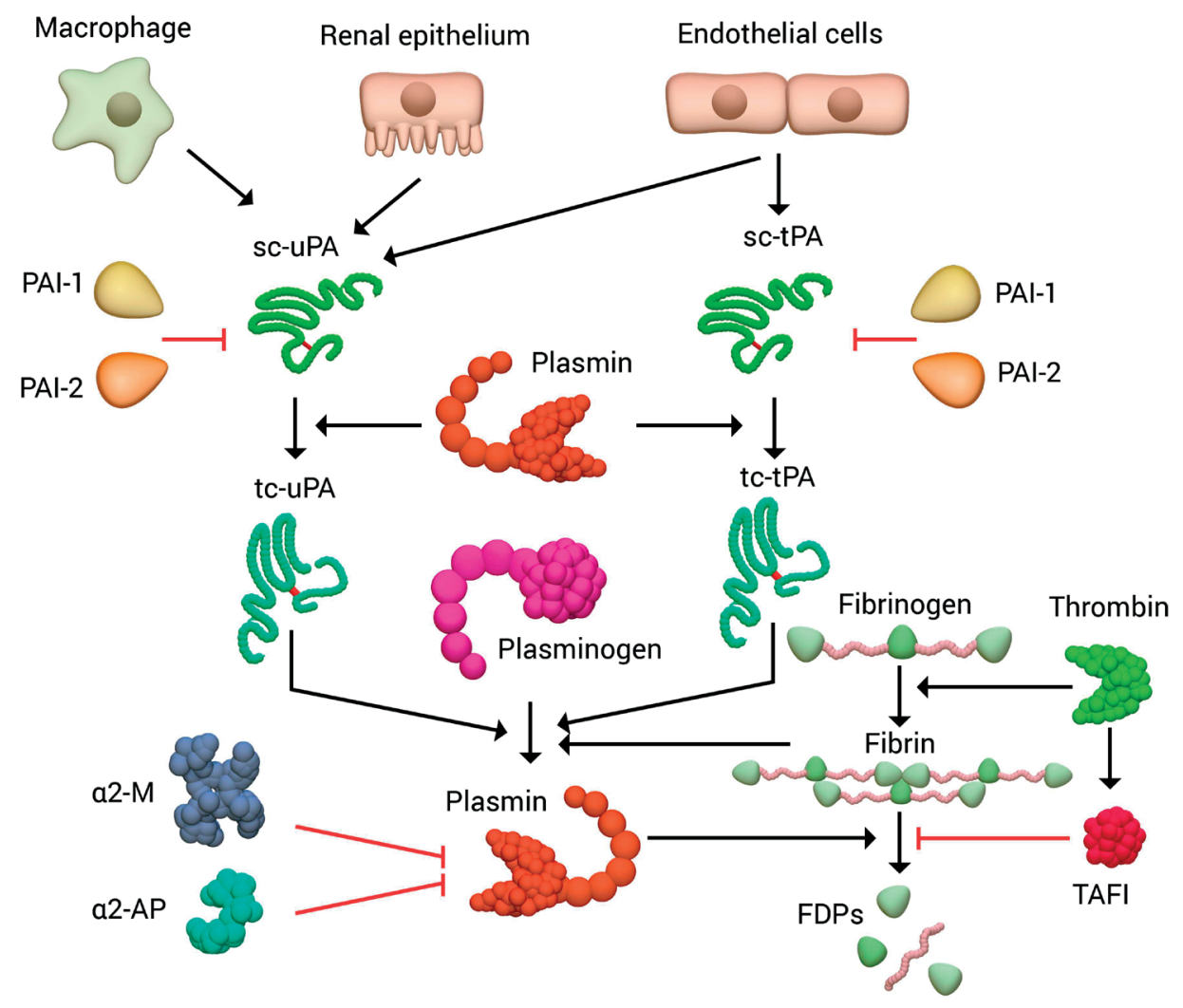

Figure 2. Overview of fibrinolysis. Single-chain (sc) tPA and UPA are secreted from endothelial cells and from renal epithelium, monocytes/macrophages or endothelial cells, respectively. Both tPA and UPA can be inhibited by plasminogen activator inhibitors (PAI-1 and PAI-2). Once plasmin is generated, it converts single chain tPA and uPA to two-chain (tc) forms. Plasminogen is converted to the active protease plasmin primarily by these tc-tPA (with fibrin as a cofactor) or tc-uPA. Plasmin cleaves fibrin to fibrin degradation products (FDPs), which can be inhibited by TAFI. Plasmin itself is inhibited by $\alpha 2$-antiplasmin ( $\alpha 2$-AP), and $\alpha 2$-macroglobulin ( $\alpha 2-M)$. 
Table 1. Plasmin substrates and corresponding cleavage sites.

\begin{tabular}{|c|c|c|}
\hline Substrate & Plasmin cleavage site & Reference \\
\hline \multirow[t]{5}{*}{ Fibrin(ogen) } & Arg104 ( $\alpha$ chain) & Walker et al. ${ }^{23} 1999$ \\
\hline & Arg42 ( $\beta$-chain) & \\
\hline & Lys133 ( $\beta$-chain) & \\
\hline & Lys62 ( $\gamma$-chain) & \\
\hline & Lys85 ( $\gamma$-chain) & \\
\hline \multirow[t]{3}{*}{ Glu-plasminogen } & Lys62 & Wiman et al. ${ }^{24} 1973$ \\
\hline & Arg68 & Wiman et al. ${ }^{25} 1973$ \\
\hline & Lys77 & Violand et al. ${ }^{26} 1976$ \\
\hline \multirow[t]{4}{*}{ Factor V } & Arg348 & Omar et al. ${ }^{16} 1987$ \\
\hline & Lys1656 & Lee et al. ${ }^{27} 1989$ \\
\hline & $\operatorname{Arg} 1765$ & Zeibdawi et al. ${ }^{28} 2001$ \\
\hline & Lys1827 & \\
\hline \multirow[t]{2}{*}{ (sc-)tPA } & Arg275 & Pennica et al..$^{29} 1983$ \\
\hline & & Johanessen et al. ${ }^{30} 1989$ \\
\hline \multirow[t]{2}{*}{ (sc-)uPA } & Lys158 & Irigoyen et al. ${ }^{31} 1999$ \\
\hline & Lys135 & Collen et al. ${ }^{21} 1991$ \\
\hline \multirow[t]{5}{*}{ TFPI } & Lys86 & Li et al. ${ }^{20} 1998$ \\
\hline & Arg107 & \\
\hline & Arg199 & \\
\hline & Lys249 & \\
\hline & Lys256 & \\
\hline Factor $X$ & Lys433 & Pryzdial et al. ${ }^{19}$ \\
\hline \multirow[t]{5}{*}{ Factor IX } & Lys43 & Samis et al. ${ }^{18} 2000$ \\
\hline & Arg145 & \\
\hline & Arg180 & \\
\hline & Lys316 & \\
\hline & $\operatorname{Arg} 318$ & \\
\hline \multirow[t]{4}{*}{ TAFI } & Arg92 & Marx et al. ${ }^{32} 2002$ \\
\hline & Arg302 & \\
\hline & Lys327 & \\
\hline & Arg330 & \\
\hline \multirow[t]{4}{*}{ Factor VIII } & Lys36 & Nogami et al. ${ }^{33} 2007$ \\
\hline & Arg336 & Ogiwara et al. ${ }^{17} 2010$ \\
\hline & Arg372 & Nishiya et al. ${ }^{34} 2010$ \\
\hline & Arg740 & \\
\hline ADAMTS13 & Unknown & Crawley et al. 222005 \\
\hline VWF & Lys1491 & Brophy et al. 2017 \\
\hline
\end{tabular}

The amino acid residue preceding cleavage site is given. sc, single-chain; tPA, tissue plasminogen activator; TFPI, tissue factor pathway inhibitor; ADAMTS13, a disintegrin-like and metalloprotease with thrombospondin type 1 repeats; VWF, von Willebrand factor. 


\section{EFFECT OF PLASMIN ON VWF}

\section{Structure and regulation of VWF}

VWF is a large plasma glycoprotein with essential functions in haemostasis. VWF circulates in plasma, at a concentration of $\sim 10 \mu \mathrm{g} / \mathrm{mL}^{35}$, in the form of multimers that comprise a varying number of VWF monomers $(250-270 \mathrm{kDa}) .{ }^{36}$ Thus, the molecular weight of VWF multimers ranges from 500 to $20,000 \mathrm{kDa}$, depending on the number of subunits. ${ }^{37}$ Each VWF monomer possesses a number of domains, namely the D'D3, A1, $A 2, A 3, D 4, C 1$ to $C 6$, and CK domains. ${ }^{38}$ Under normal blood flow conditions VWF adopts a globular conformation ${ }^{39}$ that can bind coagulation FVIII, prolonging its lifetime in the circulation. ${ }^{40,41}$

Vascular injury induces activation of endothelial cells ${ }^{42}$ and platelets ${ }^{43}$, resulting in the release of hyperreactive ultra-large (UL) VWF multimers from Weibel-Palade bodies or $\alpha$-granules, respectively (Figure 3A). These UL-VWF are subjected to rapid proteolysis by ADAMTS13. The resulting ADAMTS13-cleaved VWF multimers are less thrombotic, but can still support normal haemostasis. ${ }^{44}$ By inducing vasoconstriction, vascular injury also increases the hydrodynamic force, resulting in elongation of VWF multimers. ${ }^{45}$ Unfolded VWF can bind to subendothelial collagen (via $A 1$ and $A 3$ domains) ${ }^{39}$ and interact with platelets through GPIb ${ }^{46}$ (via VWF A1 domain ${ }^{47}$ ) which slows down the platelets and through GPIIb/IIla (via VWF C4 domain ${ }^{48}$ ) resulting in platelet activation, shape change, expression of activated integrins and secretion of autocrine agents present on platelets.

\section{Plasmin: candidate protease for VWF}

Deficiency in ADAMTS13 results in thrombotic thrombocytopenic purpura (TTP), a disorder of thrombotic microangiopathy, characterized by an abnormal persistence of ULVWF multimers. ${ }^{49}$ Similar to the pathology in systemic inflammation/sepsis, thrombotic microangiopathies such as TTP are associated with acute dysfunctional endothelial cell activation, indicated by up to fourfold increased VWF antigen (VWF:Ag) and VWF propeptide (VWFpp). ${ }^{50}$

Interestingly, TTP episodes occur sporadically in patients with hereditary ADAMTS13 deficiency ${ }^{51}$, while patients with acquired (autoantibody-mediated) ADAMTS13 deficiency can achieve clinical remission despite being severely depleted of ADAMTS13. ${ }^{52}$ Hence, ADAMTS13 levels do not correlate with disease severity during the acute phase, suggesting the existence of regulatory mechanisms/factors other than ADAMTS13 that modulate the presentation of thrombotic microangiopathy. ${ }^{52,53}$ However, during clinical remission persistent ADAMTS13 deficiency is an established risk factor for clinical relapse. $^{54,55}$ 
A

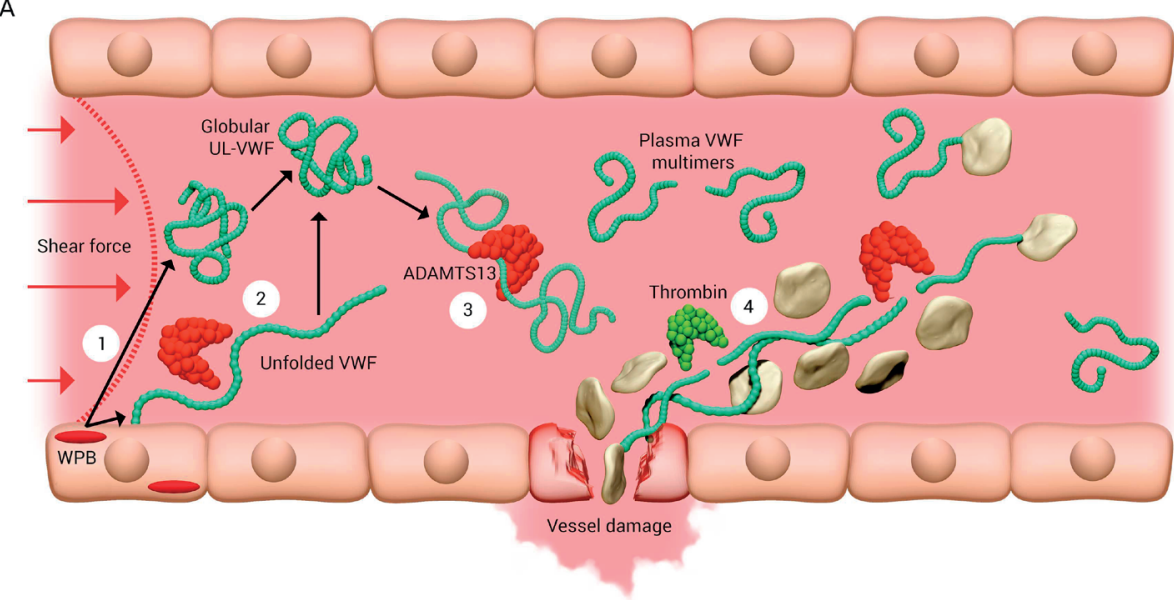

B

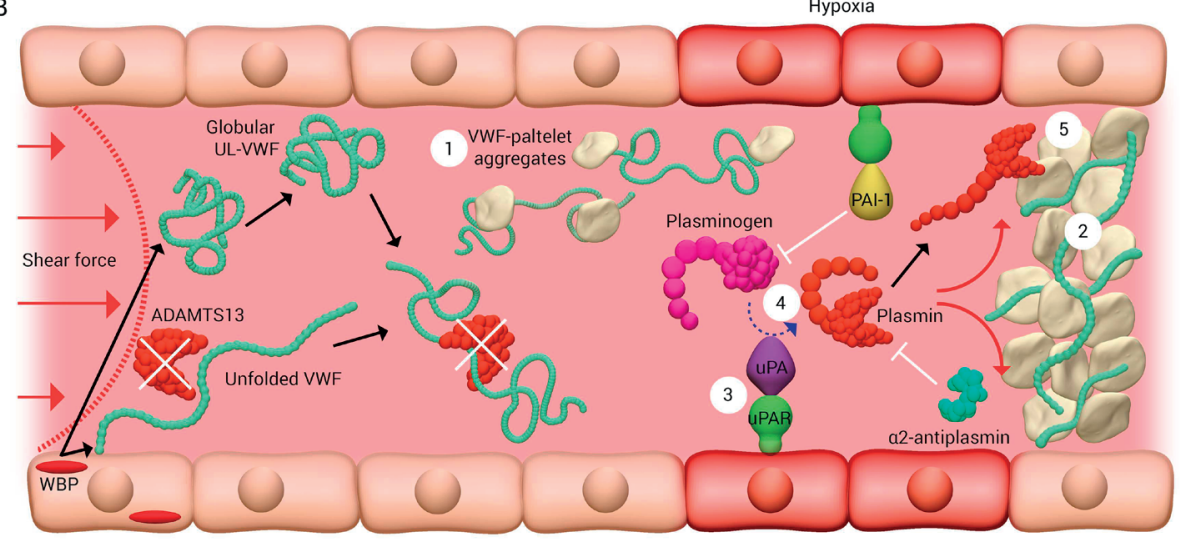

Figure 3. Model of VWF cleavage by ADAMTS13 and plasmin. (Top panel) Graphic representation of VWF proteolysis by ADAMTS13. (1) UL-VWF in a globular conformation is synthesized by the endothelium, stored within and secreted into the circulation by Weibel-Palade bodies (WPB). (2) Alternatively, a proportion of UL-VWF may remain transiently bound to the endothelial surface during exocytosis and unfold to a stretched conformation in response to shear. This conformation exposes the VWF A2 domain, enabling ADAMTS13 to cleave and release VWF into the circulation, where it adopts a globular conformation. (3) During passage through the microvasculature, globular UL-VWF in free circulation may unravel partially, allowing for cleavage of the largest, most thrombogenic multimers to smaller multimers that do not spontaneously interact with platelets. (4) At sites of vessel damage, plasma VWF will bind to exposed subendothelial collagen and subsequently, VWF unravels and recruits platelets. The presence of collagen and thrombin induces rapid platelet activation, resulting in platelet plug formation. (Bottom panel) Plasmin as an alternative protease for ADAMTS-13 to cleave VWF multimers. (1) Low or reduced ADAMTS13 levels/activity result in the loss of plasma VWF processing. Under these circumstances, platelets become bound to transiently unravelled VWF, (2) leading to accumulation of VWF-platelet aggregates that occlude the microvasculature, as seen in patients presenting with TTP. (3) Endothelial cells near the occlusion sense hypoxia and present urokinase-type plasminogen activator receptors (UPAR) on their surfaces. (4) Plasminogen is activated to plasmin by uPA bound to its receptor. Plasmin then attempts to clear the obstructed vessel by cleaving VWF in the occluding thrombus. 
Plasmin is a candidate enzyme that may serve as an alternative, or back-up, for ADAMTS13 to cleave VWF multimers. ${ }^{4}$ The concentration of plasmin in the thrombus is above $10 \mathrm{nM}$ and the environment of the thrombus protects it, at least partially, from inactivation by plasma inhibitors, most importantly $\alpha 2$-AP ${ }^{56}$. Early studies report cleavage by plasmin of the multimeric structure of VWF to lower molecular weight fragments ${ }^{57}$, mainly within disulphide loops ${ }^{58}$, resulting in loss of factor VIII ${ }^{59}$ and VWF (ristocetin cofactor) activity. ${ }^{60}$ An overview of reported effects of plasmin on VWF and the respective experimental concentrations used is given in Table 2.

\section{Mechanisms of VWF proteolysis by plasmin}

Plasmin, locally produced during fibrinolysis, can release VWF within minutes from native subendothelium. Thereby, proteolytic fragments are generated with potential activity in terms of platelet aggregation. ${ }^{61}$ VWF is a relatively poor plasmin substrate compared to fibrinogen. In fact, VWF protects fibrinogen (but not fibrin) against degradation by plasmin, thereby preserving procoagulant activity in plasma and its adhesive role in platelet thrombi. ${ }^{62}$ This might indicate that the presence of VWF in platelet-rich thrombi may contribute to their resistance to thrombolytic therapy. ${ }^{62}$

Plasmin has limited affinity for binding to and cleavage of globular VWF, but shear-3,63 or denaturant- (e.g. ristocetin) induced unfolding and consequent exposure of the VWF A1 domain strongly enhances this process. ${ }^{4}$ Of note, plasmin-binding sites and plasmin proteolysis sites within VWF are distinct. Under shear stress, conformational changes in A1A2 facilitate plasmin binding to lysine-rich regions $1405^{\mathrm{KKKK}} 1408$ in the VWF A1 domain, which subsequently enables specific plasmin cleavage at the K1491-R1492 peptide bond within the VWF A1-A2 linker region. ${ }^{3,4}$

VWF glycans play an important role in modulating VWF cleavage by plasmin. ${ }^{3,64}$ Firstly, desialylation of VWF enhances proteolysis by plasmin while it inhibits ADAMTS13 proteolysis. 3, 65, 66 Secondly, susceptibility of VWF to plasmin proteolysis at K1491-R1492 is modulated by local $\mathrm{N}$-linked glycan expression within A1A2A3. More precisely, $\mathrm{N}$-linked glycans expressed at N1515 and N1574 within the A2 domain protect VWF against proteolysis by both plasmin and ADAMTS13. 3, 67, 68 Finally, ABO(H) blood group determinants modulate VWF susceptibility to proteolysis by ADAMTS13 ( $O \geq B>A \geq A B)$ but do not influence plasmin-mediated cleavage., 69 
Table 2. Effects of plasmin on VWF; key findings per paper and concentrations of reagents used

\begin{tabular}{|c|c|c|c|c|}
\hline Year & $\begin{array}{l}\text { Primary } \\
\text { investigator }\end{array}$ & Key finding(s) & $\begin{array}{l}\text { Plasmin conc. } \\
\text { used }\end{array}$ & $\begin{array}{l}\text { VWF conc. } \\
\text { used }\end{array}$ \\
\hline 1978 & Atichartakarn ${ }^{46}$ & $\begin{array}{l}\text { Plasmin rapidly destroys the coagulant activity } \\
\text { of factor VIII but not ristocetin-cofactor activity. }\end{array}$ & $0.6 \mathrm{CU} / \mathrm{mL}$ & $\begin{array}{l}0.76 \mathrm{mg} / \mathrm{mL} \\
\text { (FVIII:VWF) }\end{array}$ \\
\hline 1979 & Henriksson ${ }^{47}$ & $\begin{array}{l}\text { Rapid loss of VWF activity upon incubation with } \\
1.96 \mathrm{CU} / \mathrm{mL} \text { plasmin. }\end{array}$ & $\begin{array}{l}0.96 \mathrm{CU} / \mathrm{mL} \\
1.92 \mathrm{CU} / \mathrm{mL}\end{array}$ & $\begin{array}{l}2.5 \mathrm{U} / \mathrm{mL} \\
\text { (FVIII:VWF) }\end{array}$ \\
\hline 1979 & Switzer ${ }^{44}$ & $\begin{array}{l}\text { Cleavage of the multimeric structure of VWF by } \\
\text { plasmin to lower molecular weight fragments }\end{array}$ & $\begin{array}{l}0.6-0.8 \mathrm{CU} / \\
\mathrm{mL}\end{array}$ & $\begin{array}{l}0.6-1.2 \\
\text { absorbance } \\
\text { units }^{1}\end{array}$ \\
\hline 1984 & Federici $^{51}$ & $\begin{array}{l}\text { Carbohydrate chains protect VWF from } \\
\text { disaggregation secondarily to proteolytic attack } \\
\text { by plasmin. }\end{array}$ & $\begin{array}{l}4 \text { and } 8 \mu g / \\
m g \text { of } V W F\end{array}$ & $\begin{array}{l}\sim 10 \mu \mathrm{g} / \\
\mathrm{mL}^{2}\end{array}$ \\
\hline 1985 & Hamilton ${ }^{45}$ & $\begin{array}{l}\text { Plasmin degrades the large VWF multimers to } \\
\text { smaller forms by cleaving within disulphide } \\
\text { loops. }\end{array}$ & $\begin{array}{l}3.2 \mathrm{mg} / \mathrm{ml} \\
(20 \mathrm{CU} / \mathrm{mg})\end{array}$ & - $2 \mathrm{mg} / \mathrm{mL}$ \\
\hline 1987 & Berkowitz $^{53}$ & $\begin{array}{l}\text { Plasmin cleaves a } 176 \mathrm{kD} \text { fragment from the } \\
\mathrm{N} \text { terminus and a } 145 \mathrm{kD} \text { fragment from the } \mathrm{C} \\
\text { terminus of the subunit. These species were } \\
\text { demonstrated in plasmas from } 4 \text { patients with } \\
\text { DVT treated with fibrinolytic agents, but not in } \\
\text { type lla VWD. }\end{array}$ & $1.8 \mathrm{mg} / \mathrm{ml}$ & $\begin{array}{l}\sim 10 \mu \mathrm{g} / \\
\mathrm{mL}^{2}\end{array}$ \\
\hline 2000 & Bonnefoy ${ }^{48}$ & $\begin{array}{l}\text { VWF is rapidly released from native } \\
\text { subendothelium when incubated with plasmin } \\
\text { on a confluent endothelial cell monolayer. The } \\
\text { released VWF is more resistant to proteolysis } \\
\text { than constitutively secreted VWF. }\end{array}$ & $\begin{array}{l}0.2 \mathrm{CU} / \mathrm{mL} \\
(200 \mathrm{nM})\end{array}$ & $6 \mu \mathrm{g} / \mathrm{mL}$ \\
\hline 2010 & Tanka-Salamon ${ }^{49}$ & $\begin{array}{l}\text { At its physiological concentration VWF is able to } \\
\text { protect fibrinogen from degradation by plasmin. }\end{array}$ & $12.5 \mathrm{nM}$ & $10 \mu \mathrm{g} / \mathrm{mL}$ \\
\hline 2012 & Wohner ${ }^{50}$ & $\begin{array}{l}\text { Plasmin at concentrations of in vivo relevance } \\
\text { resulted in extensive degradation of VWF within } \\
\text { several minutes. }\end{array}$ & $50 \mathrm{nM}$ & $10 \mu \mathrm{g} / \mathrm{mL}$ \\
\hline 2014 & Tersteeg $^{4}$ & $\begin{array}{l}\text { Efficiency of VWF cleavage by plasmin is a } \\
\text { function of its conformation: plasmin has limited } \\
\text { affinity for binding to- and cleavage of globular } \\
\text { VWF, but shear- or denaturant-mediated } \\
\text { unfolding strongly enhances this process }\end{array}$ & $\mathrm{NS}^{3}$ & $\mathrm{ND}^{4}$ \\
\hline 2017 & Brophy $^{3}$ & $\begin{array}{l}\text { Globular VWF is resistant to plasmin cleavage } \\
\text { under static conditions, but is readily cleaved by } \\
\text { plasmin under shear. } \\
\text { Plasmin cleaves the K1491-R1492 peptide } \\
\text { bond within the VWF A1-A2 linker region. } \\
\text { VWF susceptibility to plasmin proteolysis is } \\
\text { modulated by local N-linked glycan expression } \\
\text { within A1A2A3, and can be specifically inhibited } \\
\text { by heparin binding to the A1 domain. }\end{array}$ & $12.8 \mathrm{nM}$ & $10 \mu \mathrm{g} / \mathrm{ml}$ \\
\hline
\end{tabular}

${ }^{1} \mathrm{VWF}$ concentration was estimated by the absorbance at $280 \mathrm{~nm}$, corrected for light scattering, and expressed as absorbance units. ${ }^{2}$ Estimated plasma concentration of VWF. ${ }^{3} 216 \mu \mathrm{g} / \mathrm{mL}$ plasminogen $+10 \mathrm{ng} / \mathrm{mL}$ uPA or $10 \mathrm{U} / \mathrm{mL}$ streptokinase. ${ }^{4} \mathrm{VWF}$ from cultured human umbilical vein endothelial cells (HUVECs) stimulated to release VWF by addition of phorbol 12-myristate 13-acetate (PMA). Abbreviations: conc, concentration; NS, not specified; ND, not determined. 


\section{Disaggregation of VWF-platelet complexes in the presence of plasmin} In the complete absence of ADAMTS13, as in TTP, platelets as well as UL-VWF are present in the circulation (Figure $3 \mathrm{~B}$ ). However, obstructive microthrombi do not form spontaneously until a triggering event, for instance an infection, occurs. At that moment, vascular endothelial cells are the first to react on differences in oxygenation and respond to hypoxia through expression of extracellular uPA receptor. ${ }^{70}$ Subsequently, uPA-dependent plasmin formation on endothelial cells results in degradation of platelet-VWF complexes. ${ }^{4}$ Furthermore, exogenously added thrombolytic agents degrade platelet-VWF complexes with equal efficacy and can be securely controlled by the administration of lysine analogues. ${ }^{4}$ This may have important clinical implications, as thrombolytic agents may thus have therapeutic value in the treatment of TTP. ${ }^{4}$ In fact, Tersteeg et al., found that a single dose $(10 \mathrm{U} / \mathrm{mL}$, corresponding to approximately $20 \%$ of the loading dose given for pulmonary embolism) of the plasminogen (human, injected at $20 \mathrm{mg} /$ $\mathrm{kg}$ ) activator streptokinase was sufficient to attenuate symptoms of TTP and correct the thrombocytopenia in an ADAMTS13-deficient mouse model. Importantly, no evidence of bleeding or a perturbed secondary haemostasis was observed after this treatment. ${ }^{4}$ Hence, further studies on the safety and efficacy of therapeutic application of thrombolytic agents for TTP are warranted.

\section{PLASMIN AND PLATELETS}

A comprehensive overview of platelet adhesion, activation and aggregation was reviewed by Broos et al. ${ }^{71}$ Key findings on the effects of plasmin on platelets reported in literature and the experimental concentrations of plasmin used are summarized in Table 3.

\section{(In)activation of platelets by plasmin}

Under physiological conditions, plasmin activity is restricted to the proximity of the thrombus by plasma proteinase inhibitors. In stroke or deep vein thrombus formation, treatment with thrombolytic drugs can lead to free active plasmin in the circulation. ${ }^{72,73}$ Sometimes, after a successful reperfusion, re-occlusion of the damaged vessel occurs and marked platelet activation can be detected. ${ }^{21,74-79}$

In the early 70s, Niewiarowskie et al. were the first to report reversible plasmin-induced platelet aggregation, accompanied by a significant granule release. ${ }^{80,81}$ In contrast, plasmin treatment could also reduce platelet aggregation after stimulation with thrombin, collagen or ADP. ${ }^{80,82}$ These results already suggested a dual effect of plasmin depending on plasmin concentration, temperature, exposure time and platelet environment. 
Table 3. Effects of plasmin on platelets; key findings per paper and concentrations of reagents used

\begin{tabular}{|c|c|c|c|}
\hline Year & $\begin{array}{l}\text { Primary } \\
\text { Investigator }\end{array}$ & Key finding(s) & $\begin{array}{l}\text { Plasmin } \\
\text { conc. } \\
\text { used }\end{array}$ \\
\hline 1973 & Niewiarowski $^{67}$ & $\begin{array}{l}\text { Plasmin-induced platelet aggregation is reversible and is } \\
\text { accompanied by significant granule release. }\end{array}$ & $\begin{array}{l}2.2 \mathrm{CU} / \\
\mathrm{mg}\end{array}$ \\
\hline 1985 & Adelman $^{91}$ & $\begin{array}{l}\text { Treatment of washed platelets with plasmin resulted in progressive } \\
\text { loss of Gplb accompanied by loss of the agglutination response when } \\
\text { combined with ristocetin in the presence of VWF. }\end{array}$ & $1 \mathrm{CU} / \mathrm{mL}$ \\
\hline 1985 & Adelman $^{92}$ & $\begin{array}{l}\text { Plasmin reduced ristocetin-mediated agglutination of washed } \\
\text { platelets in the presence of von Willebrand factor (VWF) following a } \\
60 \text { min incubation. Plasmin treatment of washed platelets released } \\
\text { a glycocalicin-related antigen into the surrounding medium, } \\
\text { corresponding to loss of VWF-dependent, ristocetin-induced } \\
\text { agglutination. }\end{array}$ & $\begin{array}{l}0.05-1.0 \\
\mathrm{CU} / \mathrm{mL}\end{array}$ \\
\hline 1985 & Schafer $^{79}$ & $\begin{array}{l}\text { Plasmin concentrations that did not affect platelet shape change, } \\
\text { release, or aggregation (less than } 1.0 \text { caseinolytic units [CU]/ } \\
\mathrm{ml} \text { ) caused a dose- and time-dependent inhibition of platelet } \\
\text { aggregation in response to thrombin, ionophore A23187, and } \\
\text { collagen. Complete loss of aggregation occurred at } 0.1-0.5 \mathrm{CU} / \mathrm{ml} \text { of } \\
\text { plasmin. }\end{array}$ & $\begin{array}{l}<1.0 \\
\mathrm{CU} / \mathrm{mL}\end{array}$ \\
\hline 1986 & Schafer $^{70}$ & $\begin{array}{l}\text { In washed human platelets, plasmin at concentrations of } \geq 1.0 \mathrm{CU} / \mathrm{mL} \\
\text { induces aggregation. }\end{array}$ & $\begin{array}{l}\geq 1.0 \\
\mathrm{CU} / \mathrm{mL}\end{array}$ \\
\hline 1991 & Cramer $^{95}$ & $\begin{array}{l}\text { Plasmin treatment of platelets at } 37^{\circ} \mathrm{C} \text { resulted in the disappearance } \\
\text { of GPlb from the cell surface and its subsequent redistribution into } \\
\text { the channels and vesicles of the surface-connected canalicular } \\
\text { system. }\end{array}$ & $\begin{array}{l}0.2 \mathrm{CU} / \\
\mathrm{mL}\end{array}$ \\
\hline 1991 & $\mathrm{Lu}^{78}$ & $\begin{array}{l}\text { Lowering the temperature from } 37 \text { to } 22 \text { degrees } \mathrm{C} \text {, plasmin at low } \\
\text { concentrations ( } 0.1 \text { to } 0.5 \mathrm{CU} / \mathrm{mL} \text { ) fully activated platelets. When } \\
\text { platelets were treated with } 0.2 \mathrm{CU} / \mathrm{mL} \text { of plasmin, lowering the } \\
\text { temperature resulted in increased expression of fibrinogen receptors, } \\
\text { in platelet release and aggregation. }\end{array}$ & $\begin{array}{l}0.1 \text { to } \\
0.5 \mathrm{CU} / \\
\mathrm{mL}\end{array}$ \\
\hline 1992 & Gouin ${ }^{84}$ & $\begin{array}{l}\text { Incubation of human platelet-rich plasma with streptokinase does } \\
\text { not produce any detectable platelet activation but leads to a time- } \\
\text { dependent inhibition of ADP-induced aggregation accompanied by } \\
\text { substantial fibrinogenolysis. }\end{array}$ & $\mathrm{NS}^{1}$ \\
\hline 1994 & Pasche $^{83}$ & $\begin{array}{l}\text { Plasmin treatment reduced maximal reversible fibrinogen binding } \\
\text { in a dose-dependent fashion, and this reduction in binding was } \\
\text { accompanied by a correlative reduction in the maximal rate of } \\
\text { aggregation. }\end{array}$ & $\begin{array}{l}0.4-4 \\
\mathrm{CU} / \mathrm{mL}\end{array}$ \\
\hline 1995 & Loscalzo $^{74}$ & $\begin{array}{l}\text { Plasmin at higher concentrations (approximately } 1 \text { caseinolytic unit/ } \\
\mathrm{ml} \text { ), plasmin activates the platelet directly. }\end{array}$ & $\begin{array}{l}1.0 \mathrm{CU} / \\
\mathrm{mL}\end{array}$ \\
\hline 1995 & Nakamura $^{71}$ & $\begin{array}{l}\text { Plasmin produces a small rise in platelet cytosolic } \mathrm{Ca}^{2+} \text { and a tyrosine } \\
\text { kinase-dependent enhancement of } \mathrm{Ca}^{2+} \text { turnover. }\end{array}$ & $\begin{array}{l}\geq 1.0 \\
\mathrm{CU} / \mathrm{mL}\end{array}$ \\
\hline 1997 & $\begin{array}{l}\text { Kinlough- } \\
\text { Rathbone }^{80}\end{array}$ & $\begin{array}{l}\text { Incubation with plasmin almost completely inhibited thrombin- } \\
\text { induced aggregation, release of serotonin, and increase in cytosolic } \\
\mathrm{Ca}^{2+} \text {. }\end{array}$ & $\begin{array}{l}0.2 \mathrm{CU} / \\
\mathrm{ml}\end{array}$ \\
\hline 2000 & Ishii-Watabe ${ }^{72}$ & $\begin{array}{l}\text { Plasmin causes the degranulation of platelets; subsequently, ADP } \\
\text { released from granules plays a crucial role in the induction of platelet } \\
\text { aggregation. }\end{array}$ & $\begin{array}{l}\geq 1.0 \\
\mathrm{CU} / \mathrm{mL}\end{array}$ \\
\hline
\end{tabular}


Table 3. Effects of plasmin on platelets; key findings per paper and concentrations of reagents used (continued)

\begin{tabular}{llll}
\hline Year & $\begin{array}{l}\text { Primary } \\
\text { Investigator }\end{array}$ & Key finding(s) & $\begin{array}{l}\text { Plasmin } \\
\text { conc. } \\
\text { used }\end{array}$ \\
\hline 2001 & Ervin $^{77}$ & $\begin{array}{l}\text { Sustained exposure }(60 \mathrm{~min}) \text { of platelets to very low plasmin doses } \\
\text { leads to platelet activation, both at } 22 \text { and } 37 \text { degrees Celsius. The } \\
\text { resulting platelet aggregation was not accompanied by dense or } \\
\text { alpha-granule secretion. }\end{array}$ & $\begin{array}{l}0.05 \\
\mathrm{CU} / \mathrm{mL}\end{array}$ \\
& & $\begin{array}{l}\text { Desensitization of PAR1 has no effect on plasmin-induced platelet } \\
\text { aggregation PAR4 is cleaved by plasmin at the thrombin-cleavage } \\
\text { site R47. Desensitization of PAR4 completely eliminates plasmin- } \\
\text { induced aggregation. Platelets treated with a PAR4 antagonist do not } \\
\text { auinton }{ }^{88}\end{array}$ & $1 \mathrm{CU} / \mathrm{mL}$ \\
& & & \\
\hline
\end{tabular}

${ }^{1} 300 \mathrm{IU} / \mathrm{mL}$ streptokinase was added to platelet-rich plasma. Abbreviations: conc, concentration; NS, not specified.

Plasmin, at concentrations $\geq 1$ caseinolytic unit [CU]/ml causes platelet degranulation and aggregation in in vitro studies using isolated, plasma-free platelets. ${ }^{74,83-87}$ To compare, the amount of plasminogen in $1 \mathrm{~mL}$ of normal plasma corresponds to $3-4 \mathrm{CU}^{88}$, which amounts to a concentration of $20.3 \pm 2.6 \mathrm{mg} / 100 \mathrm{ml}$ or $80 \mu \mathrm{M}$ of plasmin. The clinical dose of r-tPA used for treatment of ischaemic stroke $(0.9 \mathrm{mg} / \mathrm{kg}$ for 1 hour $)$ resulted in generation of $1.6 \mathrm{CU} / \mathrm{mL}$ plasmin in a canine animal model ${ }^{89}$. More in detail, plasmin activates protein kinase C (PKC) and stimulates dose-dependent diacylglycerol (and phosphatic acid) production by phospholipase $\mathrm{C}$ activation. ${ }^{83}$ Subsequently, plasmin produces a small rise in platelet cytosolic $\mathrm{Ca}^{2+}$ and a tyrosine kinase-dependent enhancement of $\mathrm{Ca}^{2+}$ turnover. $^{83,84}$ Upon plasmin-induced platelet degranulation, ADP most likely plays a crucial role in the induction of platelet aggregation via the P2Tac receptor. ${ }^{85}$ Interestingly, prolonged (>60 $\mathrm{min}$ ) exposure of platelets to very low doses $(0.05 \mathrm{CU} / \mathrm{ml})$ of plasmin also leads to platelet aggregation and enhanced expression of procoagulant activity. ${ }^{90}$ Likewise, upon lowering the temperature to $22^{\circ} \mathrm{C}$, incubation with low concentrations of plasmin $(<0.1 \mathrm{CU} / \mathrm{ml})$ induces strong platelet aggregation. ${ }^{91}$ These results may be of clinical relevance, because the fibrinolytic system was found to be activated during cardiopulmonary bypass, in which the temperature of the patient's blood is reduced. ${ }^{91}$

Plasmin at concentrations $<1.0[\mathrm{CU}] / \mathrm{ml}$, can cause a dose- and time-dependent inhibition of platelet aggregation and thromboxane $B 2\left(T \times B_{2}\right.$, inactive metabolite of $\left.T X A_{2}\right)$ production in response to thrombin, ionophore $A 23187$ and collagen. ${ }^{74,87,92,93}$ This plasmin-induced inhibitory effect may result from a proteolysis-mediated increase in cyclic AMP ${ }^{94}$, blockage of the mobilization of arachidonic acid from membrane phospholipid pools ${ }^{92}$ and/or modification of the expression of glycoprotein receptors 
(GPIb and GPIIb/IIla, see below for more details). Interestingly, plasmin synergizes with prostaglandin 12 in inhibiting thrombin- and ADP-induced platelet activity, a finding of potential importance for the control of platelet accrual at sites of vascular injury, where the release of tPA and prostaglandin 12 by adjacent endothelial cells is generally increased. ${ }^{95}$

Of note, in plasma, the main effect of plasmin is impairment of aggregation. ${ }^{96,97}$ However, this is not related to a direct effect of plasmin on platelets. Plasmin degrades fibrinogen and fibrin/fibrinogen degradation products bind to fibrinogen and/or GPIIb/ IIIla receptors, both resulting in a reduction of the formation of fibrinogen bridges between platelets. ${ }^{98}$ Additionally, in plasma, plasmin is inhibited by $\alpha 2$-antiplasmin. ${ }^{99}$

\section{Plasmin activates platelets via PAR4}

Plasmin cleaves both thrombin receptors PAR1 and PAR4 on platelets. ${ }^{100,}{ }^{101}$ Cleavage by plasmin was reported to be 1700 -fold lower relative to thrombin cleavage. ${ }^{102,103}$ Four cleavage sites have been identified on the PAR1 exodomain (R41, R70, K76 and K82). R41 is the thrombin-cleavage site and generates a transiently activated exodomain. The $\mathrm{R} 70, \mathrm{~K} 76$ and $\mathrm{K} 82$ sites are located on a linker region that tethers the ligand to the body of the receptor. However, the high affinity of plasmin for R70, K76 and K82 and the low affinity for R41 (thrombin-cleavage site) results predominantly in inactivation of PAR1 by truncating the tethered ligand at the distal sites. ${ }^{100}$ Additionally, desensitization of PAR1 had no effect on plasmin-induced platelet aggregation. ${ }^{101}$ PAR4, on the other hand, is cleaved by plasmin at the thrombin-cleavage site R47. ${ }^{101}$ As a result, desensitization of PAR4 completely eliminated aggregation in response to plasmin, and platelets treated with a PAR4 antagonist, as well as platelets isolated from PAR4 homozygous null mice, failed to aggregate in response to plasmin. Altogether, Quinton et al. concluded that plasmin induces platelet aggregation primarily through slow cleavage of PAR4. ${ }^{101}$

\section{Plasmin induces reversible translocation of platelet GPIb}

The addition of plasmin to washed platelets results in progressive loss of GPIb as measured by fluorescence flow cytometry and by loss of ristocetin-induced VWF-dependent platelet agglutination. ${ }^{104,105}$ The loss of platelet surface GPIb, initially assigned to plasmin-mediated cleavage of $\mathrm{GPI}^{105-107}$, is now known to be related to a redistribution of GPIb, which moves from the external membrane into the surface-connected canalicular system. ${ }^{108}$ This translocation of GPIb is related to actin polymerization, as cytochalasin D (a specific inhibitor of actin polymerization) inhibited the migration of GPIb after plasmin stimulation. ${ }^{108}$ Subsequently, neutralization of plasmin by the inhibitors aprotinin and tripeptide Val-Phe- $\mathrm{Lys}-\mathrm{CH}_{2} \mathrm{Cl}$ permitted a time-dependent recovery of platelet agglutination. ${ }^{109}$ The functional recovery was accompanied by a restoration 
of a normal amount of GPIb on the platelet surface. This suggests a reverse translocation of the GPIb molecules initially present at the cell surface. ${ }^{109}$

\section{Effect of plasmin on GPIIb/IIla}

Plasmin exerts opposing effects on GPIIb/IIla, resulting in enhanced or impaired fibrinogen binding and platelet aggregation. On the one hand, plasmin-induced platelet activation and granule release results in the expression of active GPIIb/IIla receptors on the cell surface. ${ }^{110}$ On the other hand, in plasma, plasmin degrades GPIIla by cleaving a proteolytically susceptible loop domain, but only after degradation of the solubilized GPIIb/IIla by Staphylococcus aureus V8 (Glu-C) endoprotease. ${ }^{96}$ In contrast, treatment of isolated platelets with plasmin has no effect on GPIIb/IIla expression. ${ }^{108}$ These findings suggest that, in plasma, plasmin modifies GPIIla by a unique proteolytic event that is dependent on fibrinogen binding and, consequently, is accompanied by significant reduction in fibrinogen binding and aggregation. ${ }^{96}$

\section{CONCLUSION}

Although the mechanism of plasmin generation and its function in fibrinolysis are rather well-defined, the precise (patho)physiological effects of plasmin on other components of the coagulation cascade are hitherto not completely understood. In this review we provide an overview of the available evidence on the effects of plasmin on VWF and platelets. Summarizing, it can be stated that, in addition to ADAMTS13, plasmin can regulate the thrombogenicity of VWF through its protease activity. This role of plasmin appears particularly important in pathologies in which high-molecular-weight VWF multimers accumulate, as exemplified by TTP. Moreover, although plasmin-mediated platelet activation is unlikely to play a significant role in normal haemostasis, during pharmacological thrombolysis where the plasmin activity could reach as high as 1 CU/ $\mathrm{ml}$, it may have implications in terms of vessel patency and effectiveness of treatment. Hence, future research should focus on obtaining a better understanding of the molecular mechanisms of plasmin binding to- and cleavage of VWF as well as of plasmin's effects on platelet activation and aggregation. For experiments with platelets, particular attention should be given to simulating the in vivo environment as much as possible in the experimental conditions to obtain reliable results. Together, this knowledge on the interaction of plasmin, VWF and platelets will support the ongoing development of novel therapies ${ }^{111,112}$ as well as the use of existing thrombolytic therapy to potentially prevent the occurrence of- or degrade existing VWF-platelet aggregates in conditions such as TTP. 


\section{REFERENCES}

1. Longstaff C, Kolev K. Basic mechanisms and regulation of fibrinolysis. J Thromb Haemost. 2015;13 Suppl 1:S98-105.

2. Cesarman-Maus G, Hajjar KA. Molecular mechanisms of fibrinolysis. Br J Haematol. 2005;129(3):307-21.

3. Brophy TM, Ward SE, McGimsey TR, Schneppenheim S, Drakeford C, O'Sullivan JM, et al. Plasmin Cleaves Von Willebrand Factor at K1491-R1492 in the A1-A2 Linker Region in a Shearand Glycan-Dependent Manner In Vitro. Arterioscler Thromb Vasc Biol. 2017;37(5):845-55.

4. Tersteeg $C$, de Maat $S$, De Meyer SF, Smeets MW, Barendrecht AD, Roest M, et al. Plasmin cleavage of von Willebrand factor as an emergency bypass for ADAMTS13 deficiency in thrombotic microangiopathy. Circulation. 2014;129(12):1320-31.

5. Raum D, Marcus D, Alper CA, Levey R, Taylor PD, Starzl TE. Synthesis of human plasminogen by the liver. Science. 1980;208(4447):1036-7.

6. Hajjar KA. The molecular basis of fibrinolysis. In: Hematology of Infancy and Childhood (ed. by Nathan D.G., Orkin S.H., Ginsburg D. \& Look A.T.). Philadelphia, USA: W.B. Saunders Co.; 2003. p. 1497-514.

7. Forsgren M, Raden B, Israelsson M, Larsson K, Heden LO. Molecular cloning and characterization of a full-length cDNA clone for human plasminogen. FEBS Lett. 1987;213(2):254-60.

8. Holvoet $P$, Lijnen HR, Collen D. A monoclonal antibody specific for Lys-plasminogen. Application to the study of the activation pathways of plasminogen in vivo. J Biol Chem. 1985;260(22):12106-11.

9. Ny T, Sawdey M, Lawrence D, Millan JL, Loskutoff DJ. Cloning and sequence of a cDNA coding for the human beta-migrating endothelial-cell-type plasminogen activator inhibitor. Proc Natl Acad Sci U S A. 1986;83(18):6776-80.

10. Ye RD, Wun TC, Sadler JE. cDNA cloning and expression in Escherichia coli of a plasminogen activator inhibitor from human placenta. J Biol Chem. 1987;262(8):3718-25.

11. Schaller J, Gerber SS. The plasmin-antiplasmin system: structural and functional aspects. Cell Mol Life Sci. 2011;68(5):785-801.

12. Thorsen S, Clemmensen I, Sottrup-Jensen L, Magnusson S. Adsorption to fibrin of native fragments of known primary structure from human plasminogen. Biochim Biophys Acta. 1981;668(3):377-87.

13. Medved L, Nieuwenhuizen W. Molecular mechanisms of initiation of fibrinolysis by fibrin. Thromb Haemost. 2003;89(3):409-19.

14. Redlitz A, Tan AK, Eaton DL, Plow EF. Plasma carboxypeptidases as regulators of the plasminogen system. J Clin Invest. 1995;96(5):2534-8.

15. Aoki N, Moroi M, Tachiya K. Effects of alpha2-plasmin inhibitor on fibrin clot lysis. Its comparison with alpha2-macroglobulin. Thromb Haemost. 1978;39(1):22-31.

16. Omar MN, Mann KG. Inactivation of factor Va by plasmin. J Biol Chem. 1987;262(20):97505.

17. Ogiwara K, Nogami K, Nishiya K, Shima M. Plasmin-induced procoagulant effects in the blood coagulation: a crucial role of coagulation factors $\mathrm{V}$ and VIII. Blood Coagul Fibrinolysis. 2010;21(6):568-76.

18. Samis JA, Ramsey GD, Walker JB, Nesheim ME, Giles AR. Proteolytic processing of human coagulation factor IX by plasmin. Blood. 2000;95(3):943-51. 
19. Pryzdial EL, Lavigne N, Dupuis N, Kessler GE. Plasmin converts factor $X$ from coagulation zymogen to fibrinolysis cofactor. J Biol Chem. 1999;274(13):8500-5.

20. Li A, Wun TC. Proteolysis of tissue factor pathway inhibitor (TFPI) by plasmin: effect on TFPI activity. Thromb Haemost. 1998;80(3):423-7.

21. Collen D, Lijnen HR. Basic and clinical aspects of fibrinolysis and thrombolysis. Blood. 1991;78(12):3114-24.

22. Crawley JT, Lam JK, Rance JB, Mollica LR, O'Donnell JS, Lane DA. Proteolytic inactivation of ADAMTS13 by thrombin and plasmin. Blood. 2005;105(3):1085-93.

23. Walker JB, Nesheim ME. The molecular weights, mass distribution, chain composition, and structure of soluble fibrin degradation products released from a fibrin clot perfused with plasmin. J Biol Chem. 1999;274(8):5201-12.

24. Wiman B. Primary structure of peptides released during activation of human plasminogen by urokinase. Eur J Biochem. 1973;39(1):1-9.

25. Wiman B, Wallen P. Activation of human plasminogen by an insoluble derivative of urokinase. Structural changes of plasminogen in the course of activation to plasmin and demonstration of a possible intermediate compound. Eur J Biochem. 1973;36(1):25-31.

26. Violand BN, Castellino FJ. Mechanism of the urokinase-catalyzed activation of human plasminogen. J Biol Chem. 1976;251(13):3906-12.

27. Lee $C D$, Mann KG. Activation/inactivation of human factor $V$ by plasmin. Blood. 1989;73(1):185-90.

28. Zeibdawi AR, Pryzdial EL. Mechanism of factor Va inactivation by plasmin. Loss of A2 and A3 domains from a Ca2+-dependent complex of fragments bound to phospholipid. J Biol Chem. 2001;276(23):19929-36.

29. Pennica D, Holmes WE, Kohr WJ, Harkins RN, Vehar GA, Ward CA, et al. Cloning and expression of human tissue-type plasminogen activator CDNA in E. coli. Nature. 1983;301(5897):21421.

30. Johannessen M, Nielsen F, Petersen LC. Plasmin-catalysed cleavage of single chain tissuetype plasminogen activator in fibrin clots. Fibrinolysis. 1989;3(4):215-20.

31. Irigoyen JP, Munoz-Canoves $P$, Montero L, Koziczak M, Nagamine $Y$. The plasminogen activator system: biology and regulation. Cell Mol Life Sci. 1999;56(1-2):104-32.

32. Marx PF, Dawson PE, Bouma BN, Meijers JC. Plasmin-mediated activation and inactivation of thrombin-activatable fibrinolysis inhibitor. Biochemistry. 2002;41(21):6688-96.

33. Nogami K, Shima M, Matsumoto T, Nishiya K, Tanaka I, Yoshioka A. Mechanisms of plasmincatalyzed inactivation of factor VIII: a crucial role for proteolytic cleavage at Arg336 responsible for plasmin-catalyzed factor VIII inactivation. J Biol Chem. 2007;282(8):5287-95.

34. Nishiya K, Nogami K, Okada K, Matsuo O, Takeyama M, Ogiwara K, et al. Determination of a factor VIII-interactive region within plasmin responsible for plasmin-catalysed activation and inactivation of factor VIII(a). Thromb Haemost. 2010;104(1):105-17.

35. Peyvandi F, Garagiola I, Baronciani L. Role of von Willebrand factor in the haemostasis. Blood Transfus. 2011;9 Suppl 2:s3-8.

36. Wagner DD. Cell biology of von Willebrand factor. Annu Rev Cell Biol. 1990;6:217-46.

37. Furlan M. Von Willebrand factor: molecular size and functional activity. Ann Hematol. 1996;72(6):341-8.

38. Zhou YF, Eng ET, Zhu J, Lu C, Walz T, Springer TA. Sequence and structure relationships within von Willebrand factor. Blood. 2012;120(2):449-58. 
39. Schneider SW, Nuschele S, Wixforth A, Gorzelanny C, Alexander-Katz A, Netz RR, et al. Shearinduced unfolding triggers adhesion of von Willebrand factor fibers. Proc Natl Acad Sci U S A. 2007;104(19):7899-903.

40. Ruggeri ZM. Role of von Willebrand factor in platelet thrombus formation. Ann Med. 2000;32 Suppl 1:2-9.

41. Owen WG, Wagner RH. Antihemophilic factor: separation of an active fragment following dissociation by salts or detergents. Thromb Diath Haemorrh. 1972;27(3):502-15.

42. Dong JF, Moake JL, Nolasco L, Bernardo A, Arceneaux W, Shrimpton CN, et al. ADAMTS-13 rapidly cleaves newly secreted ultralarge von Willebrand factor multimers on the endothelial surface under flowing conditions. Blood. 2002;100(12):4033-9.

43. Liu L, Choi H, Bernardo A, Bergeron AL, Nolasco L, Ruan C, et al. Platelet-derived VWFcleaving metalloprotease ADAMTS-13. J Thromb Haemost. 2005;3(11):2536-44.

44. Savage $B$, Sixma J], Ruggeri ZM. Functional self-association of von Willebrand factor during platelet adhesion under flow. Proc Natl Acad Sci U S A. 2002;99(1):425-30.

45. Savage B, Saldivar E, Ruggeri ZM. Initiation of platelet adhesion by arrest onto fibrinogen or translocation on von Willebrand factor. Cell. 1996;84(2):289-97.

46. Vicente V, Kostel PJ, Ruggeri ZM. Isolation and functional characterization of the von Willebrand factor-binding domain located between residues His1-Arg293 of the alpha-chain of glycoprotein Ib. J Biol Chem. 1988;263(34):18473-9.

47. Fujimura Y, Titani K, Holland LZ, Russell SR, Roberts JR, Elder JH, et al. von Willebrand factor. A reduced and alkylated 52/48-kDa fragment beginning at amino acid residue 449 contains the domain interacting with platelet glycoprotein lb. J Biol Chem. 1986;261(1):381-5.

48. Plow EF, Pierschbacher MD, Ruoslahti E, Marguerie GA, Ginsberg MH. The effect of Arg-GlyAsp-containing peptides on fibrinogen and von Willebrand factor binding to platelets. Proc Natl Acad Sci U S A. 1985;82(23):8057-61.

49. Tsai HM, Lian EC. Antibodies to von Willebrand factor-cleaving protease in acute thrombotic thrombocytopenic purpura. N Engl J Med. 1998;339(22):1585-94.

50. van Mourik JA, Boertjes R, Huisveld IA, Fijnvandraat K, Pajkrt D, van Genderen PJ, et al. von Willebrand factor propeptide in vascular disorders: $A$ tool to distinguish between acute and chronic endothelial cell perturbation. Blood. 1999;94(1):179-85.

51. Crawley JT, Scully MA. Thrombotic thrombocytopenic purpura: basic pathophysiology and therapeutic strategies. Hematology Am Soc Hematol Educ Program. 2013;2013:292-9.

52. Zheng XL, Kaufman RM, Goodnough LT, Sadler JE. Effect of plasma exchange on plasma ADAMTS13 metalloprotease activity, inhibitor level, and clinical outcome in patients with idiopathic and nonidiopathic thrombotic thrombocytopenic purpura. Blood. 2004;103(11):4043-9.

53. Furlan M, Robles R, Galbusera M, Remuzzi G, Kyrle PA, Brenner B, et al. von Willebrand factor-cleaving protease in thrombotic thrombocytopenic purpura and the hemolyticuremic syndrome. N Engl J Med. 1998;339(22):1578-84.

54. Jin M, Casper TC, Cataland SR, Kennedy MS, Lin S, Li YJ, et al. Relationship between ADAMTS13 activity in clinical remission and the risk of TTP relapse. Br J Haematol. 2008;141(5):651-8.

55. Peyvandi F, Lavoretano S, Palla R, Feys HB, Vanhoorelbeke K, Battaglioli T, et al. ADAMTS13 and anti-ADAMTS13 antibodies as markers for recurrence of acquired thrombotic thrombocytopenic purpura during remission. Haematologica. 2008;93(2):232-9. 
56. Kolev K, Lerant I, Tenekejiev K, Machovich R. Regulation of fibrinolytic activity of neutrophil leukocyte elastase, plasmin, and miniplasmin by plasma protease inhibitors. J Biol Chem. 1994;269(25):17030-4.

57. Switzer ME, McKee PA. Immunologic studies of native and modified human factor VIII/von Willebrand factor. Blood. 1979;54(2):310-21.

58. Hamilton KK, Fretto LJ, Grierson DS, McKee PA. Effects of plasmin on von Willebrand factor multimers. Degradation in vitro and stimulation of release in vivo. J Clin Invest. 1985;76(1):261-70.

59. Atichartakarn V, Marder VJ, Kirby EP, Budzynski AZ. Effects of enzymatic degradation on the subunit composition and biologic properties of human factor VIII. Blood. 1978;51(2):28197.

60. Henriksson P, Nilsson IM. Effects of leukocytes, plasmin and thrombin on clotting factors. A comparative in vitro study. Thromb Res. 1979;16(3-4):301-12.

61. Bonnefoy A, Legrand C. Proteolysis of subendothelial adhesive glycoproteins (fibronectin, thrombospondin, and von Willebrand factor) by plasmin, leukocyte cathepsin G, and elastase. Thromb Res. 2000;98(4):323-32.

62. Tanka-Salamon A, Kolev K, Machovich R, Komorowicz E. Proteolytic resistance conferred to fibrinogen by von Willebrand factor. Thromb Haemost. 2010;103(2):291-8.

63. Wohner N, Kovacs A, Machovich R, Kolev K. Modulation of the von Willebrand factordependent platelet adhesion through alternative proteolytic pathways. Thromb Res. 2012;129(4):e41-6.

64. Federici AB, Elder JH, De Marco L, Ruggeri ZM, Zimmerman TS. Carbohydrate moiety of von Willebrand factor is not necessary for maintaining multimeric structure and ristocetin cofactor activity but protects from proteolytic degradation. J Clin Invest. 1984;74(6):2049-55.

65. McGrath RT, McKinnon TA, Byrne B, O'Kennedy R, Terraube V, McRae E, et al. Expression of terminal alpha2-6-linked sialic acid on von Willebrand factor specifically enhances proteolysis by ADAMTS13. Blood. 2010;115(13):2666-73.

66. Berkowitz SD, Federici AB. Sialic acid prevents loss of large von Willebrand factor multimers by protecting against amino-terminal proteolytic cleavage. Blood. 1988;72(5):1790-6.

67. McKinnon TA, Chion AC, Millington AJ, Lane DA, Laffan MA. N-linked glycosylation of VWF modulates its interaction with ADAMTS13. Blood. 2008;111(6):3042-9.

68. Nowak AA, Canis K, Riddell A, Laffan MA, McKinnon TA. O-linked glycosylation of von Willebrand factor modulates the interaction with platelet receptor glycoprotein lb under static and shear stress conditions. Blood. 2012;120(1):214-22.

69. Bowen DJ. An influence of $A B O$ blood group on the rate of proteolysis of von Willebrand factor by ADAMTS13. Journal of thrombosis and haemostasis : JTH. 2003;1(1):33-40.

70. Graham CH, Fitzpatrick TE, McCrae KR. Hypoxia stimulates urokinase receptor expression through a heme protein-dependent pathway. Blood. 1998;91(9):3300-7.

71. Broos K, Feys HB, De Meyer SF, Vanhoorelbeke K, Deckmyn H. Platelets at work in primary hemostasis. Blood Rev. 2011;25(4):155-67.

72. Califf RM, Fortin DF, Tenaglia AN, Sane DC. Clinical risks of thrombolytic therapy. The American journal of cardiology. 1992;69(2):12A-20A.

73. Schweizer J, Kirch W, Koch R, Elix H, Hellner G, Forkmann L, et al. Short- and long-term results after thrombolytic treatment of deep venous thrombosis. Journal of the American College of Cardiology. 2000;36(4):1336-43.

74. Coller BS. Platelets and thrombolytic therapy. N Engl J Med. 1990;322(1):33-42. 
75. Fitzgerald DJ, Catella F, Roy L, FitzGerald GA. Marked platelet activation in vivo after intravenous streptokinase in patients with acute myocardial infarction. Circulation. 1988;77(1):142-50.

76. Ohlstein EH, Storer B, Fujita T, Shebuski RJ. Tissue-type plasminogen activator and streptokinase induce platelet hyperaggregability in the rabbit. Thrombosis research. 1987;46(4):575-85.

77. Kerins DM, Roy L, FitzGerald GA, Fitzgerald DJ. Platelet and vascular function during coronary thrombolysis with tissue-type plasminogen activator. Circulation. 1989;80(6):1718-25.

78. Fitzgerald DJ, Wright $F$, FitzGerald GA. Increased thromboxane biosynthesis during coronary thrombolysis. Evidence that platelet activation and thromboxane A2 modulate the response to tissue-type plasminogen activator in vivo. Circulation research. 1989;65(1):83-94.

79. Watabe A, Ohta M, Matsuyama N, Mizuno K, el Borai N, Tanimoto T, et al. Characterization of plasmin-induced platelet aggregation. Research communications in molecular pathology and pharmacology. 1997;96(3):341-52.

80. Niewiarowski S, Senyi AF, Gillies P. Plasmin-induced platelet aggregation and platelet release reaction. Effects on hemostasis. The Journal of clinical investigation. 1973;52(7):1647-59.

81. Niewiarowski S, Gurewich V, Senyi AF, Mustard JF. The effect of fibrinolysis on platelet function. Thrombosis et diathesis haemorrhagica Supplementum. 1971;47:99.

82. Miller JL, Katz AJ, Feinstein MB. Plasmin inhibition of thrombin-induced platelet aggregation. Thrombosis et diathesis haemorrhagica. 1975;33(2):286-309.

83. Schafer Al, Maas AK, Ware JA, Johnson PC, Rittenhouse SE, Salzman EW. Platelet protein phosphorylation, elevation of cytosolic calcium, and inositol phospholipid breakdown in platelet activation induced by plasmin. The Journal of clinical investigation. 1986;78(1):739.

84. Nakamura K, Kimura M, Fenton JW, 2nd, Andersen TT, Aviv A. Duality of plasmin effect on cytosolic free calcium in human platelets. The American journal of physiology. 1995;268(4 Pt 1):C958-67.

85. Ishii-Watabe A, Uchida E, Mizuguchi H, Hayakawa T. On the mechanism of plasmin-induced platelet aggregation. Implications of the dual role of granule ADP. Biochemical pharmacology. 2000;59(11):1345-55.

86. Pasche B, Loscalzo J. Platelets and fibrinolysis. Platelets. 1991;2(3):125-34.

87. Loscalzo J, Pasche B, Ouimet $\mathrm{H}$, Freedman JE. Platelets and plasminogen activation. Thrombosis and haemostasis. 1995;74(1):291-3.

88. Friberger P. Synthetic peptide substrate assays in coagulation and fibrinolysis and their application on automates. Semin Thromb Hemost. 1983;9(4):281-300.

89. Rudd MA, George D, Amarante P, Vaughan DE, Loscalzo J. Temporal effects of thrombolytic agents on platelet function in vivo and their modulation by prostaglandins. Circ Res. 1990;67(5):1175-81.

90. Ervin AL, Peerschke El. Platelet activation by sustained exposure to low-dose plasmin. Blood coagulation \& fibrinolysis : an international journal in haemostasis and thrombosis. 2001;12(6):415-25.

91. Lu H, Soria C, Cramer EM, Soria J, Maclouf J, Perrot JY, et al. Temperature dependence of plasmin-induced activation or inhibition of human platelets. Blood. 1991;77(5):996-1005.

92. Schafer Al, Adelman B. Plasmin inhibition of platelet function and of arachidonic acid metabolism. The Journal of clinical investigation. 1985;75(2):456-61. 
93. Kinlough-Rathbone RL, Perry DW, Rand ML, Packham MA. Pretreatment of human platelets with plasmin inhibits responses to thrombin, but potentiates responses to low concentrations of aggregating agents, including the thrombin receptor activating peptide, SFLLRN. Thrombosis and haemostasis. 1997;77(4):741-7.

94. Adnot S, Ferry N, Hanoune J, Lacombe ML. Plasmin: a possible physiological modulator of the human platelet adenylate cyclase system. Clinical science (London, England : 1979). 1987;72(4):467-73.

95. Gresele P, Kleiman NS, Lopez JA, Page CP. Platelets in Thrombotic and Non-Thrombotic Disorders. 1 ed: Springer International Publishing; 2017.

96. Pasche B, Ouimet $\mathrm{H}$, Francis $\mathrm{S}$, Loscalzo J. Structural changes in platelet glycoprotein Ilb/IIla by plasmin: determinants and functional consequences. Blood. 1994;83(2):404-14.

97. Gouin I, Lecompte T, Morel MC, Lebrazi J, Modderman PW, Kaplan C, et al. In vitro effect of plasmin on human platelet function in plasma. Inhibition of aggregation caused by fibrinogenolysis. Circulation. 1992;85(3):935-41.

98. de Haan J, Schonberger J, Haan J, van Oeveren W, Eijgelaar A. Tissue-type plasminogen activator and fibrin monomers synergistically cause platelet dysfunction during retransfusion of shed blood after cardiopulmonary bypass. The Journal of thoracic and cardiovascular surgery. 1993;106(6):1017-23.

99. Blockmans D, Deckmyn H, Hove LV, Vermylen J. The effect of plasmin on platelet function. Platelets. 1996;7(3):139-48.

100. Kuliopulos A, Covic L, Seeley SK, Sheridan PJ, Helin J, Costello CE. Plasmin desensitization of the PAR1 thrombin receptor: kinetics, sites of truncation, and implications for thrombolytic therapy. Biochemistry. 1999;38(14):4572-85.

101. Quinton TM, Kim S, Derian CK, Jin J, Kunapuli SP. Plasmin-mediated activation of platelets occurs by cleavage of protease-activated receptor 4 . The Journal of biological chemistry. 2004;279(18):18434-9.

102. Kimura M, Andersen TT, Fenton JW, 2nd, Bahou WF, Aviv A. Plasmin-platelet interaction involves cleavage of functional thrombin receptor. The American journal of physiology. 1996;271(1 Pt 1):C54-60.

103. Parry MA, Myles T, Tschopp J, Stone SR. Cleavage of the thrombin receptor: identification of potential activators and inactivators. The Biochemical journal. 1996;320 ( Pt 1):335-41.

104. Adelman B, Michelson AD, Handin RI, Ault KA. Evaluation of platelet glycoprotein lb by fluorescence flow cytometry. Blood. 1985;66(2):423-7.

105. Adelman B, Michelson AD, Loscalzo J, Greenberg J, Handin RI. Plasmin effect on platelet glycoprotein lb-von Willebrand factor interactions. Blood. 1985;65(1):32-40.

106. Adelman B, Michelson AD, Greenberg J, Handin RI. Proteolysis of platelet glycoprotein lb by plasmin is facilitated by plasmin lysine-binding regions. Blood. 1986;68(6):1280-4.

107. Kamat SG, Michelson AD, Benoit SE, Moake JL, Rajasekhar D, Hellums JD, et al. Fibrinolysis inhibits shear stress-induced platelet aggregation. Circulation. 1995;92(6):1399-407.

108. Cramer EM, Lu H, Caen JP, Soria C, Berndt MC, Tenza D. Differential redistribution of platelet glycoproteins Ib and IIb-IIla after plasmin stimulation. Blood. 1991;77(4):694-9.

109. Lu H, Soria C, Soria J, De Romeuf C, Perrot JY, Tenza D, et al. Reversible translocation of glycoprotein Ib in plasmin-treated platelets: consequences for platelet function. European journal of clinical investigation. 1993;23(12):785-93. 
110. Rabhi-Sabile S, Pidard D. Exposure of human platelets to plasmin results in the expression of irreversibly active fibrinogen receptors. Thrombosis and haemostasis. 1995;73(4):693701.

111. Peyvandi F, Callewaert F. Caplacizumab for Acquired Thrombotic Thrombocytopenic Purpura. N Engl J Med. 2016;374(25):2497-8.

112. Callewaert F, Roodt J, Ulrichts H, Stohr T, van Rensburg WJ, Lamprecht S, et al. Evaluation of efficacy and safety of the anti-VWF Nanobody ALX-0681 in a preclinical baboon model of acquired thrombotic thrombocytopenic purpura. Blood. 2012;120(17):3603-10. 



$$
\text { Chapter } 10
$$




\section{Summary, discussion and future prospects}





\section{SUMMARY, DISCUSSION AND FUTURE PROSPECTS}

This thesis is centred around the role of VWF in its GP1b $\alpha$-binding conformation, called active VWF, in haemostasis: on the one hand in healthy individuals (Chapter 3 ), on the other hand in conditions that throw off the haemostatic balance and hence result in thrombosis (Chapter 4-7) or bleeding (Chapter 8). However, haemostasis is a highly complex process that is contingent on a plethora of interactions between plasma coagulation (and fibrinolytic) factors, platelets and the endothelium. Many factors, such as inflammatory cytokines and intravascular shear stress, also affect an individual's tendency to clot or bleed. Because of this multifactorial nature of the haemostatic process, we not only assessed active VWF levels, but also the quantity and functioning of platelets and other plasma coagulation/fibrinolytic proteins in the conditions that we studied.

Figure 1 presents a schematic overview of the studies described in this thesis. This concluding chapter summarizes and discusses the most important findings of this thesis, and presents several suggestions for future research.

\section{Inhibition of platelet-VWF interactions by the S-VWFA1 VHH}

Since both platelets and our S-VWFA1 VHH bind to an epitope in the VWF A1 domain, the $\mathrm{VHH}$ could potentially interfere with the interaction between platelets and active VWF. Therefore, we studied the effects of the VHH on platelet-VWF binding in several functional assays, of which the results were presented in Chapter 2. In line with our hypothesis, the VHH (dose-dependently) abrogated binding of platelets to active VWF (induced by ristocetin or a VWD type 2B mutation) in a flow cytometric assay and light transmission aggregometry. However, surprisingly, in a perfusion assay and the PFA$200 \circledR$, S-VWFA1 could not inhibit the adhesion of platelets to VWF, or only at very high concentrations (>50 $\mu \mathrm{g} / \mathrm{mL}$ ). Platelet GPIb $\alpha$ binding to collagen-bound VWF in a static assay also remained unaffected by S-VWFA1.

\section{Collagen-binding potentially modulates S-VWFA1 efficacy}

We considered several factors that may explain the differential effects of the VHH in various functional assays, including the presence of shear stress, ristocetin and rapid dilution in "open" systems such as the perfusion set-up and PFA-200@ assay. However, our observations, combined with previous findings by Hulstein et al. ${ }^{1}$, argued against these factors as critical determinants of the inhibiting efficacy of the $\mathrm{VHH}$. Collagen immobilization, on the other hand, has a high potential to disturb the binding of our $\mathrm{VHH}$ to the active conformation of VWF. This hypothesis is supported by the lack of inhibition of platelet binding in assays that were dependent on VWF-collagen type I binding (perfusion assay, PFA-200®) or collagen type III-binding (static collagen binding assay). 


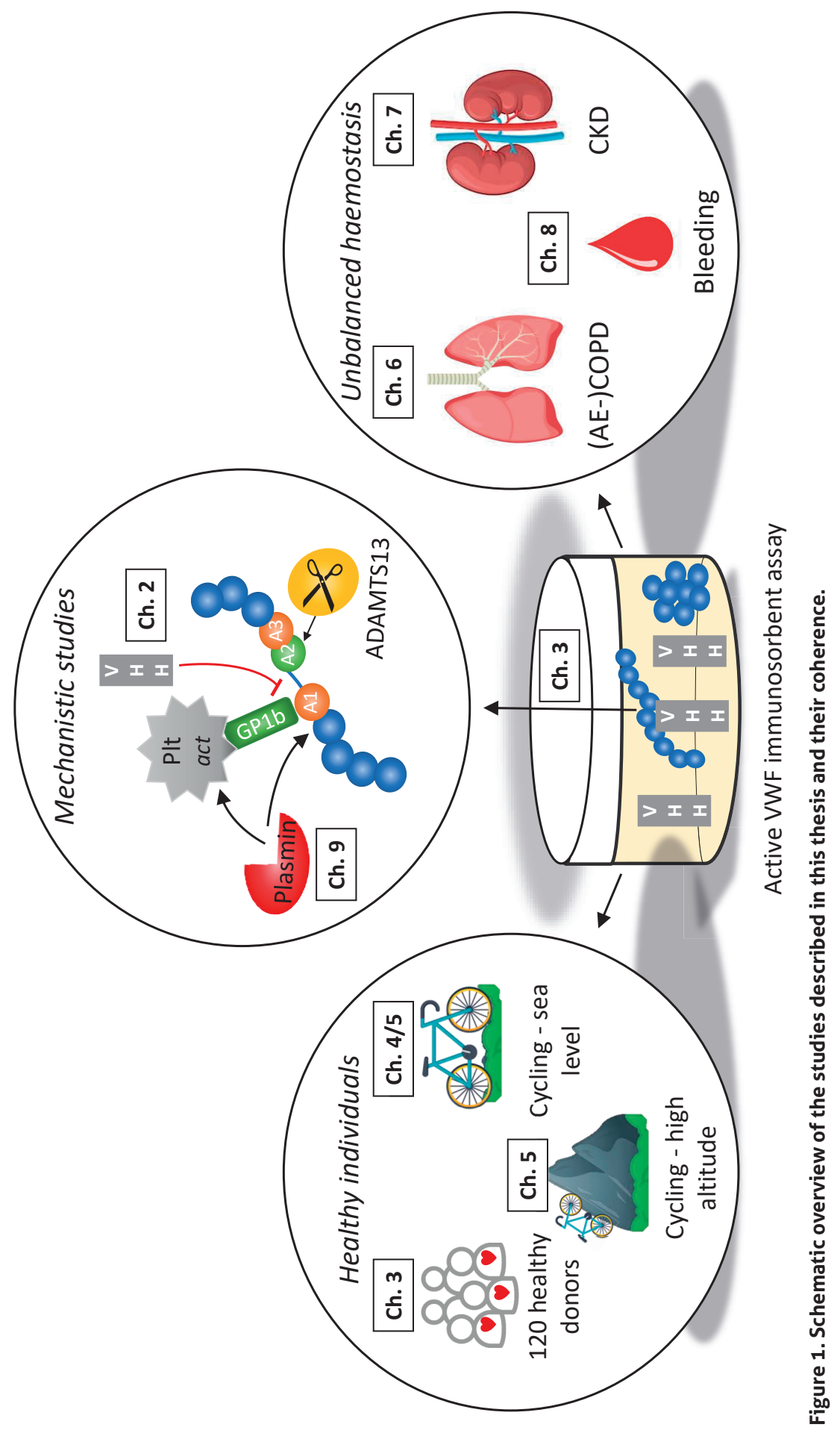


VWF contains multiple binding sites for collagen: the $A 1$ domain contains binding sites for collagen types I, III, and $\mathrm{VI}^{2-4}$, while its homologous $\mathrm{A} 3$ domain binds to collagen types I and III. ${ }^{5-7}$ In physiological conditions, upon immobilization at sites of vascular injury via high-affinity $A 3$ domain-collagen (I/III) interactions, ${ }^{8}$ shear forces unfold VWF to expose its $A 1$ domains. The $A 1$ domain can simultaneously interact with collagen and platelet GPIb $\alpha$, allowing platelet recruitment under flow conditions. ${ }^{4}$ Importantly, A1 binding to collagen was previously shown to induce a conformational change in A1. ${ }^{4,9}$ This conformation has a higher affinity for GPIb $\alpha$, resulting in a more stable VWFGPIb $\alpha$ bond. ${ }^{9}$ We hypothesize that this conformational change may be responsible for blocking access to the $\mathrm{VHH}$ epitope and thus preventing inhibition of platelet-binding to collagen-immobilized VWF. Altogether, we conclude that the S-VWFA1 VHH only inhibits platelet binding to active VWF in solution (i.e. circulating active VWF), but not platelet binding to collagen-bound active VWF.

\section{S-VWFA1-mediated inhibition of microthrombi formation in TTP?}

If the above-described hypothesis holds true in further experimental (animal) studies, it implies that S-VWFA1 has potentially beneficial properties as a therapeutic biological for conditions with increased circulating active VWF. As such, it may be able to prevent pathological platelet-VWF aggregate formation while leaving physiological haemostasis at sites of vascular injury (where VWF immobilizes to collagen) intact. For example, in acute TTP exacerbations, caused by binding of platelets to accumulated active UL-VWF multimers (due to deficient ADAMTS13-mediated proteolysis), blocking the VWF-platelet interactions would be a promising strategy. The mainstay of acute TTP treatment is plasma exchange in conjunction with immunosuppression. ${ }^{10}$ Plasma exchange removes UL-VWF and the causative auto-antibodies while replenishing functional ADAMTS-13. However, long-term plasma exchange required in patients with refractory or relapsing TTP is tremendously expensive and debilitating for these patients. ${ }^{11}$

Other groups have previously explored the therapeutic use of agents with the ability to block VWF-platelet binding (Table 1). ${ }^{12-14}$ Caplacizumab, a bivalent VHH (Nanobody®) targeting the VWF A1 domain is the most successful, as it has recently been approved (under the commercial name Cablivi®) by both the FDA (2019) and EMA (2018) for use in patients with acute TTP. The efficacy and safety of caplacizumab were evaluated in the phase II TITAN study (75 acute TTP patients) and the follow-up phase III HERCULES trial (145 acute TTP patients). ${ }^{10,15,16}$ Both studies demonstrated that caplacizumab treatment resulted in faster normalization of platelet counts, fewer exacerbations and lower incidence of major thromboembolic events and death from TTP compared to treatment with placebo. ${ }^{15,16}$ However, caplacizumab treatment was also associated with an increased risk of (mucocutaneous) bleeding. ${ }^{15}$ This bleeding tendency can be 
explained by the increased clearance rate of the caplacizumab-VWF-FVIII complex. ${ }^{17}$ Additionally, bleeding may be related to the mechanism of action of caplacizumab, as it blocks the interaction of all sizes and activation stages of multimeric VWF with platelet $\mathrm{Gplb}^{14}$ including VWF that is bound to collagen. The resulting mucocutaneous bleeding mimics the phenotype of VWD. ${ }^{18}$ As mentioned, our in vitro findings described in Chapter 2 suggest that the S-VWFA1 VHH may hypothetically perform better in this regard, as it does not seem to block physiological primary haemostasis (i.e. platelet binding to collagen-bound VWF).

Table 1. Previously developed inhibitors of the VWF-GPIb $\alpha$ interaction.

\begin{tabular}{|c|c|c|c|}
\hline Name & Type & Models in which agent was tested & Ref. \\
\hline \multirow[t]{2}{*}{ AJvW-2 } & \multirow[t]{2}{*}{ Murine monoclonal antibody } & In vitro - platelets & 19,20 \\
\hline & & Ex vivo - guinea pigs/dogs & 21,22 \\
\hline \multirow[t]{2}{*}{ AJW200 } & \multirow{2}{*}{$\begin{array}{l}\text { Humanized monoclonal } \\
\text { antibody }\end{array}$} & In vitro - platelets & 23 \\
\hline & & Ex vivo - monkeys/rabbits/ dogs & 23,24 \\
\hline \multirow[t]{6}{*}{ ARC1779 } & \multirow[t]{6}{*}{ Aptamer } & In vitro - platelets & 12 \\
\hline & & Ex vivo - pig aorta segments & 12 \\
\hline & & In vivo-monkeys & 12 \\
\hline & & Phase I/II - TTP patients & $25-27$ \\
\hline & & Phase II - Patients undergoing carotid endarterectomy & 28 \\
\hline & & Phase II - VWD type 2B patients & 29 \\
\hline \multirow[t]{2}{*}{ ARC15105 } & \multirow[t]{2}{*}{ Aptamer } & In vitro/ex vivo - platelets/pig aorta segments & 30 \\
\hline & & In vivo-monkeys & 30 \\
\hline \multirow{7}{*}{$\begin{array}{l}\text { ALX-0081/0681 } \\
\text { (Caplacizumab) }\end{array}$} & \multirow[t]{7}{*}{ Bivalent nanobody } & In vitro - platelets & 31 \\
\hline & & Ex vivo- $\mathrm{PCl}$ patients' platelets & 32 \\
\hline & & In vivo - baboons & 14 \\
\hline & & Phase I- healthy humans & 31 \\
\hline & & Phase $\mathrm{lb}$ - angina patients- $\mathrm{PCl}$ & 31 \\
\hline & & Phase II - acute TTP patients & 15 \\
\hline & & Phase III - acute TTP patients & 10,16 \\
\hline \multirow[t]{2}{*}{ BT200 } & \multirow[t]{2}{*}{ Aptamer } & In vitro-platelets, VWF monkeys & 33 \\
\hline & & In vivo - monkeys & 33 \\
\hline
\end{tabular}

Abbreviations: VWD, von Willebrand disease; TTP, thrombotic thrombocytopenic purpura; $\mathrm{PCl}$, percutaneous coronary intervention.

\section{Animal studies and epitope mapping for S-VWFA1}

To demonstrate whether the S-VWFA1 VHH can indeed resolve intravascular plateletVWF aggregate formation without causing bleeding complications, its efficacy is currently being evaluated in a collaborative animal study using a TTP (ADAMTS13 ${ }^{-1-}$ ) mouse model. ${ }^{34}$ Of note, given the very small size of $\mathrm{VHHs}( \pm 15 \mathrm{kDa}$, i.e. ten times smaller than monoclonal antibodies), S-VWFA1 is expected to be rapidly cleared by glomerular filtra- 
tion. Extension of the half-life in the circulation may be achieved by fusion to albumin, thereby limiting renal elimination. ${ }^{35}$ Depending on the results in this murine animal model, the S-VWFA1 should also be tested in a preclinical monkey/baboon model ${ }^{14}$, as there are inter-species differences in haemostasis and other physiological variables between rodents and non-rodents. ${ }^{36}$ If animal studies would show good stability (in terms of half-life/clearance) and promising inhibition of platelet-VWF aggregation without increased bleeding risk, clinical studies may follow. Appendix I (Valorisation) further elaborates on the considerations that should be taken into account before doing so.

Another missing puzzle piece in our hypothesis is the exact binding epitope of S-VWFA1 in the VWF A1 domain. Mapping of this epitope with commonly employed strategies, such as X-ray co-crystallography of the antigen-antibody complex, is less suitable because binding of the $\mathrm{VHH}$ depends on the structural conformation of VWF. ${ }^{37}$ However, hydrogen/deuterium exchange mass spectrometry (HDX-MS) epitope mapping technology is compatible with conformational epitopes in the solution state. ${ }^{38}$ The principle is that binding of an antibody reduces the solvent accessibility of antigen residues in the binding interface, thereby decreasing the level of deuterium incorporation in peptic fragments containing the affected residues. ${ }^{38}$ Therefore, comparing the HDX profiles between antibody-free and antibody-bound states can map the antibody epitopes in antigens. ${ }^{39}$ Collaborative HDX-MS studies ${ }^{40}$ for epitope mapping of the VHH to the VWF A1 domain are currently in progress.

\section{Quantification of active VWF in plasma samples}

Besides its potential use as a therapeutic agent, we explored whether the S-VWFA1 VHH could be used to quantify active VWF in plasma samples. The most important question before initiating these studies was: why would we want to measure active VWF in the first place? The answer is that active VWF levels provide specific information about the fraction of circulating VWF that can directly bind platelets. As such, quantification of active VWF levels can hypothetically provide additional clues on the pathophysiology of conditions associated with bleeding (except mild bleeding without thrombocytopenia, as shown in Chapter 8) or thrombosis. It is important to emphasize that quantification of VWF antigen can never be replaced by active VWF. Globular, latent VWF itself is functional in many relevant ways: as a carrier of FVIII, a marker of previous or ongoing endothelial activation and, depending on the situation (shear stress, endothelial damage), this "inactive" fraction can quickly become activated to bind platelets. ${ }^{41}$

An immunosorbent assay for active VWF already existed before we initiated our studies. ${ }^{1}$ However, no technical validation of this assay had been published, and its reference ranges were not determined. This hampered, in our opinion, the application of the active 
VWF assay by other research groups and implementation in laboratory practice. After all, to determine whether a condition is associated with significantly divergent active VWF levels, one first needs to establish what values to expect in a healthy population. ${ }^{42}$ Therefore, in Chapter 3 of this thesis, we reported on the technical validation of the ELISA assay for active VWF (based on the S-VWFA1 VHH). The validation experiments showed that our assay is specific for active VWF, is reproducible (intra- and inter-assay CVs between $2.4-7.2 \%$ and $4.1-9.4 \%$ ) and linear in the range between $90.1-424.5 \%$ (normalized to normal pooled plasma, NPP). Subsequently, we established its reference range by measuring active VWF levels in 120 healthy adults. Active VWF values in this population correlated significantly with other, established VWF assays, and the reference range was determined to be $91.6-154.8 \%$ of NPP. ${ }^{43}$

Of note, results of our assay are normalized to values for NPP on the same plate, which is neither ideal for interpretation of results nor for comparability of results between laboratories (see also Appendix I). The reason we chose to express active VWF in this unit is that there is no reference material to calibrate with. In theory, it would be possible to calculate the absolute concentration of active VWF using recombinant, mutated active VWF as a standard, but for that two assumptions need to be made, namely: (1) the VHH recognizes active VWF with an activating mutation (e.g. R1306W) similarly as native, non-mutated active VWF, in both healthy individuals and in a variety of pathological conditions and (2) the multimer composition, which will vary between individuals, does not affect the recognition by our VHH. Because we think these assumptions cannot be made "safely" at the moment, we propose that expressing active VWF as a \% of NPP is the most practical approach for now.

Having established the variation in active VWF in healthy individuals (at rest), we next sought to investigate conditions that could affect circulating active VWF levels.

\section{Effects of (repeated) strenuous exercise and hypoxia on haemostasis}

The exercise paradox

Physical exercise is known to prevent cardiovascular disease, reduce all-cause mortality and is therefore generally considered and promoted to be important for a healthy lifestyle. ${ }^{44,45}$ However, there are also numerous reports of exercise-related thrombotic events ${ }^{46,47}$, and the risk of sudden cardiac death is 2 to 3 -fold higher in athletes compared to non-athletes. ${ }^{48}$ This phenomenon is called the exercise paradox. ${ }^{49}$ Physical exercise is known to affect haemostasis in various ways, amongst others by activating the endothelium, increasing thrombin generation and platelet activation, thus inducing a transient prothrombotic state. ${ }^{50}$ Much less is known on the short-term effects of re- 
peated bouts of exercise on coagulation. Hypothetically, this repetition could condition the haemostatic response.

\section{Repeated exercise exhausts the endothelial response}

In Chapter 4, we summarized our findings from a thorough literature search on the effects of repeated exercise on haemostasis. Although not typically part of a literature review, this chapter also presents new data from a small-scale cycling study, in which five male healthy volunteers cycled $80 \mathrm{~km}$ at sea level on three consecutive days. Previous studies demonstrated that endothelial activation is a key contributing factor to the increased thrombotic tendency following strenuous exercise. ${ }^{50-52}$ Interestingly, we found that the exercise-induced increases in FVIII, VWF antigen, VWF propeptide and active VWF were smaller on each subsequent cycling day. ${ }^{53}$ This suggests either adaptation of the endothelial response to exercise or exhaustion of the endothelial cells VWF storage. The increased active VWF levels may be partly explained by the increased shear stress, which unfolds VWF to its platelet-binding conformation. The exercise-induced increase in VWF was accompanied by increased $\mathrm{FVIII}^{51}$, which in turn resulted in a higher thrombin generation peak. In contrast, platelet activation, measured as P-selectin expression after stimulation with various agonists, actually decreased, thereby compensating at least partly for the endothelium-induced prothrombotic tendency. Over the course of the three cycling days, the platelet response did not recover to baseline, indicating exhaustion of the platelet response as a result of repeated exercise.

In Chapter 5 we reported a second cycling study, situated at high altitude instead of sea level. This setting at 3,375 m altitude introduced the influence of hypoxia (decreased partial oxygen pressure). The results confirmed the importance of endothelial activation, as apparent from the increased VWF (level and activation status), increased FVIII levels and thrombin generation, in agreement with previous cycling studies. ${ }^{53,54}$ Normoxic exercise decreased P-selectin expression and did not affect $\alpha$ llb $\beta 3$ activation, while the latter was decreased at high altitude, confirming a previous study. ${ }^{55}$

Based on the literature and our findings on the short-term effects of repeated cycling on coagulation, we concluded that strenuous exercise induces a transient prothrombotic state, particularly in untrained individuals, that is primarily driven by the endothelial release of VWF. ${ }^{53}$ However, repeated exercise appears to induce adaptation and/or exhaustion of this prothrombotic response, supporting its favourable effects on cardiovascular health and mortality in regularly exercising individuals. ${ }^{44,45}$ In addition, our results indicate that hypoxia attenuates the exercise-induced prothrombotic state, mainly through reducing platelet activation and platelet-dependent thrombin generation. Previous studies also reported that a hypoxic training regimen attenuated the 
haemostatic response to exercise. ${ }^{56}$ Acute hypoxia is generally considered to benefit physical performance by preconditioning, as it enables ventilatory acclimatisation and raises the oxygen-carrying haemoglobin levels. ${ }^{57,58}$ Not surprisingly, altitude training is a popular way of preparation for cyclists.

\section{Exposure to exercise and hypoxia in COPD patients}

An example of a condition that is accompanied by both low oxygen pressure and physical stress is chronic obstructive pulmonary disease (COPD). COPD is characterized by persistent airflow limitation that is usually progressive. In addition, COPD is associated with an enhanced chronic inflammatory response in the airways and lung to noxious particles and gases, among which cigarette smoke. ${ }^{59}$ Exercise is an essential component of pulmonary rehabilitation programs, with the aim to control and alleviate symptoms and optimize functional capacity of COPD patients. ${ }^{60}$ Exhaustive exercise was previously found to intensify the systemic inflammation that is chronically present in COPD patients and result in increased oxidative stress. ${ }^{61}$ On the other hand, exercise is also beneficial for COPD patients, by improving exercise tolerance and training muscles. ${ }^{62}$ Thus, it is desirable to identify effective strategies to reduce the negative inflammatory effects of exercise in these patients. Interestingly, while oxygen therapy has been postulated to increase oxidative stress and inflammation in septic patients (see above) ${ }^{63}$, it was shown to prevent exercise-induced oxidative stress and attenuate the inflammatory IL- 6 response in COPD patients. ${ }^{64}$ Therefore, short-term supplementary oxygen may be beneficial to enhance training intensity and relieve dyspnoea in COPD patients during rehabilitation programs, although it is not known whether these effects remain upon frequent exercise.

\section{Standardization of exercise protocols and knowledge gaps}

Results in the literature on this topic are often inconsistent, likely due to heterogeneous study populations (especially variation in training status) as well as activity-related factors such as intensity, duration, and mode of exercise. ${ }^{53}$ Thus, standardization and precise description of exercise protocols are prerequisites for future studies to allow for comparison of results and replication by other groups. Furthermore, there are a number of gaps in knowledge that remain to be addressed. One of the most important questions relates to obtaining better estimates of the impact of training status on the incidence of exercise-induced thrombotic events, and how this compares to the effects of therapeutic intervention. Related to this, the effect of training status on the platelet response to exercise remains to be further elucidated. While there is abundant data showing that physical exercise induces platelet activation, ${ }^{51}$ we observed the opposite in both of our cycling studies. This difference may be attributed to training status, as our studies only included physically fit individuals, and a previous study also observed that 
strenuous exercise does not induce platelet hyperreactivity in trained individuals. ${ }^{65}$ Furthermore, differences in the effects of exercise between men and women need to be addressed. Interestingly, a previous cycling study by our group found that platelet reactivity increased following exercise, but this effect was stronger in men than in women. ${ }^{54}$ Since the small-scale studies described in Chapter $\mathbf{4}$ and $\mathbf{5}$ only included male participants (to not increase variation within the small group even more), it would be interesting to study differences in haemostatic responses between the sexes in a larger follow-up study. ${ }^{54}$

\section{Active VWF in conditions associated with thrombosis and bleeding}

After studying active VWF in healthy individuals in the first two parts of this thesis (Chapters 3-5), we proceeded to investigate the role of active VWF in two inflammatory conditions associated with a thrombotic phenotype and patients with a bleeding tendency.

\section{COPD}

\section{Acute exacerbations of COPD induce a prothrombotic state}

Chapter 6 reported on a prospective observational cohort study in patients with COPD. Cardiovascular events occur frequently in COPD patients ${ }^{66,67}$ and the risk of (arterial and venous) thrombotic events is known to increase during and shortly after acute exacerbations (AE-COPD). ${ }^{68-71}$ Given the extensive crosstalk between inflammation and coagulation ${ }^{72}$, we hypothesized that the heightened inflammatory state during an AE-COPD may contribute to the observed prothrombotic phenotype. In our study population, we found increased circulating platelet-monocyte complexes (PMCs) during an AE-COPD, corroborating a previous smaller study. ${ }^{73}$ Remarkably, although the formation of PMCs is considered a marker of platelet activation ${ }^{72}$, we did not observe changes in (unstimulated or agonist-stimulated) platelet P-selectin expression and $\alpha$ llb $\beta 3$ activation during an AE-COPD. This discrepancy suggests that PMC formation may represent a more sensitive marker of platelet activation, ${ }^{74}$ as platelets rapidly shed P-selectin from their surface but continue to function. ${ }^{75}$ Alternatively, PMC formation in AE-COPD may be primarily driven by monocyte activation (Mac-1 expression). Platelets and monocytes can interact in several ways, amongst others via (1) platelet P-selectin binding to monocyte PSGL-1, (2) monocyte Mac-1 binding to platelet $\alpha$ llb $\beta 3$-bound fibrinogen and (3) monocyte Mac-1 binding to platelet GPIb $\alpha .^{72}$ Given the lack of observed changes in P-selectin expression and $\alpha$ llb $\beta 3$ activation, our findings suggest that binding of Mac-1 to platelet GPIb $\alpha$ may be particularly important for PMC formation during AE-COPD. ${ }^{76}$ Mac- 1 expression can be driven by inflammatory mediators such as IL- $6^{77}$ and CRP ${ }^{78}$, and previous studies have also suggested that during inflammation the interaction of GPIb $\alpha$ and Mac- 1 is crucial for PMC formation. ${ }^{76,79}$ 
Secondly, we found that besides VWF:Ag and VWF:RCo ${ }^{80,81}$, active VWF is also elevated during an AE-COPD. Mechanistically, the increased inflammation during AE-COPD can cause elevated active VWF through saturating ADAMTS13 with the excessive VWF released from the endothelium and/or through IL-6, which limits ADAMTS-13 function, both preventing active UL-VWF cleavage. ${ }^{82}$

Thirdly, the TG peak, ETP and velocity were significantly increased during AE-COPD, while D-dimer levels and the time until clot formation did not change. Thus, the increased thrombin generation potential during an AE-COPD seems to serve predominantly to stabilize clots through TAFI and FXIII activation. ${ }^{83}$ Altogether, elevated circulating PMCs, (active) VWF and thrombin generation contribute to the prothrombotic state of COPD patients during an acute exacerbation, particularly in patients with increased systemic inflammation.

In fact, systemic inflammation affects up to $70 \%$ of patients with COPD. ${ }^{84}$ Large prospective studies in COPD patients have shown that systemic inflammation predicts a greater decline in lung function, a greater tendency to infective exacerbations and risk of mortality. ${ }^{84-86}$ Our study contributes that AE-COPD patients with systemic inflammation (CRP>10 mg/L) have a clearly increased prothrombotic tendency compared to patients with CRP levels within the reference range. Unfortunately, our study was not powered to detect significant associations between the investigated prothrombotic biomarkers and the occurrence of cardiovascular outcomes, nor could we find clinical risk factors for the increase in thrombotic markers. Nonetheless, the observed "pan-thrombotic" state suggests that AE-COPD patients may benefit from anti-thrombotic treatment.

\section{Anti-platelet and/or anti-thrombotic therapy in AE-COPD patients}

Our finding that AE-COPDs are associated with increased PMC formation is clinically relevant as PMCs are known to contribute to the development of cardiovascular disease, amongst others through their role in atherosclerotic plaque formation and instability. ${ }^{72,87}$ In addition, given the observed increased PMC formation and the association between increased platelet activation during $A E-C O P D$ and exacerbation relapse, antiplatelet therapy may be beneficial in COPD patients. ${ }^{88} \mathrm{P}_{2} \mathrm{Y}_{1} 12$ inhibitors, such as clopidogrel and ticagrelor, were previously found to decrease platelet-monocyte interaction as well as systemic inflammation. ${ }^{89,90}$ However, a recent double-blind randomised controlled trial in COPD patients found no platelet response to antiplatelet therapy with aspirin and ticagrelor in nearly one-third of COPD patients and no clinically significant reduction of inflammatory biomarkers after 6 months. ${ }^{91}$ These results emphasize the clinical heterogeneity among these patients and support that besides antiplatelet therapy, an 
antithrombotic therapy might be beneficial to reduce the cardiovascular risk in these patients.

Current guidelines recommend prophylactic administration of low-molecular-weight heparin (LMWH) on hospital admission of AE-COPD patients with reduced mobility ( $87 \%$ in our study) to prevent venous thromboembolism (VTE). ${ }^{92}$ Although LMWHs are primarily used as an anticoagulant, they also show anti-inflammatory properties and may thus have potential therapeutic effects on all (AE-)COPD patients (regardless of mobility). ${ }^{93,94} \mathrm{~A}$ recent meta-analysis on 12 RCTs investigating effects of LMWH on COPD exacerbations concluded that the addition of subcutaneous LMWH to conventional therapy may significantly improve the levels of lung function parameters and significantly reduced the risk of thrombosis, at the cost of increased minor bleeding. ${ }^{95}$ Of note, there are currently no studies on the potential benefit of other anticoagulants, such as the direct oral anticoagulants (DOACs), in AE-COPD patients.

\section{Chronic kidney disease}

\section{Chronic inflammation inflicts endothelial damage}

The second condition that we investigated is chronic kidney disease (CKD), which is defined as a reduced glomerular filtration rate (GFR) or elevated albuminuria present for at least three months. ${ }^{96}$ This condition affects $13.4 \%$ of the global population, with the most common causes being diabetes and hypertension. ${ }^{97}$ CKD is associated with an increased risk of death and arterial cardiovascular events including stroke, coronary disease, heart failure and peripheral arterial disease ${ }^{98}$, but also with venous thromboembolism (VTE). ${ }^{99,100}$ The reasons that CKD patients are at increased risk for VTE are not well understood but are likely related to the heightened inflammatory state that accompanies CKD. Many factors contribute to the inflammatory status in CKD, including increased production of proinflammatory cytokines, oxidative stress and acidosis, chronic and recurrent infections and altered metabolism of adipose tissue. ${ }^{101}$ Two main sources of inflammatory cytokines are circulating monocytes and endothelial cells. ${ }^{102}$ Since the kidney receives $25 \%$ of the entire blood volume ${ }^{103}$ but does not possess the same anti-inflammatory defence mechanisms as the liver, the kidney is a vulnerable target for inflammation-inflicted damage to the vasculature. ${ }^{101}$ The consequent damage to the endothelial barrier function mediates activation of the coagulation system, amongst others by exposure of subendothelial collagen and tissue factor and release of large amounts of VWF. ${ }^{83}$ Therefore, we hypothesized that levels of active VWF would also be increased in this condition and that the stage of kidney disease and the treatment modality may differentially affect (active) VWF levels. 


\section{Increased active VWF and VWF antigen in CKD patients}

In Chapter $\mathbf{7}$ we described the results of a collaborative study based on a cohort comprising CKD patients not receiving haemodialysis ( $C K D, n=25)$ patients with end-stage renal disease undergoing thrice-weekly haemodialysis (HD-ESRD, $n=13$ ), patients with ESRD on peritoneal dialysis (PD-ESRD, $\mathrm{n}=4)$ and healthy volunteers $(H V, n=31)$ as controls. Active VWF and VWF antigen levels in plasma from all groups were elevated compared to healthy controls, corroborating previous reports for VWF antigen. ${ }^{104,105}$ However, only for active VWF a trend with increased disease severity was observed, although this was non-significant due to the small size of the patient groups. Mechanistically, more severe stages of kidney disease are associated with progressive damage and/or activation of the endothelium and hence release of increasingly large amounts of active UL-VWF into the circulation. ${ }^{105}$ In combination with a decreased ADAMTS13 level (due to increased renal protein loss ${ }^{106}$ ) and possibly IL- 6 mediated inhibition of ADAMTS13 activity ${ }^{82}$, the excess UL-VWF will not be proteolyzed and remains in the circulation in its active conformation.

\section{(Active) VWF for cardiovascular risk prediction in CKD patients?}

Clinically, high plasma VWF levels were previously found to be a predictor of microalbuminuria, cardiovascular complications and mortality in both diabetic and non-diabetic CKD patients. ${ }^{107}$ Interestingly, we found that active VWF levels appeared to proportionally increase with progressive stages of renal failure in our study population. Therefore, we hypothesize that active VWF may be even more strongly associated with the risk of cardiovascular disease in these patients than VWF antigen levels. VWF does not only exert its procoagulant effect through the interaction of its active form with platelets but also stabilizes FVIII. Interestingly, the Multiple Environmental and Genetic Assessment of risk factors for venous thrombosis (MEGA) study, a case-control study of patients with VTE and controls, identified that the relationship between CKD and VTE could be explained by VWF and FVIII. ${ }^{108}$ Current guidelines for the diagnosis and monitoring of CKD patients recommend measuring the estimated glomerular filtration rate (eGFR) and albumin/creatinine ratio, as these are independent predictors of cardiovascular events (and decrease in renal function) ${ }^{109}$. Despite their apparently strong relation with cardiovascular risk in CKD patients, VWF and FVIII do not have a role as predicting biomarkers in these guidelines.

\section{No role for active VWF in patients with a mild bleeding diathesis}

Spontaneous interaction between increased active VWF and platelets can also tilt the haemostatic balance to the bleeding site. While active VWF has been well-studied in VWD type $2 \mathrm{~B}^{110,111}$, it was unknown whether the fraction of active VWF also plays a role in patients with a mild bleeding diathesis (without thrombocytopenia). In Chapter 
8 we reported that active VWF levels, determined in a population of 109 patients with mild bleeding symptoms, were on average relatively lower than the reference range, but this difference was not significant. In addition, active VWF was not different between individuals with and without a significant clinical bleeding score (ISTH-BAT), nor between individuals with and without VWD in their differential diagnosis. Therefore, we concluded that quantification of active VWF does not have added diagnostic value besides the established VWF antigen and ristocetin cofactor activity assays in patients with a mild bleeding diathesis.

\section{Directions for future research on coagulation in COPD and CKD Larger sample size}

Two common limitations of the studies described above are small sample size and few follow-up measurements over a relatively short period of time. Thus, these factors should be taken into account in the design of future studies on prothrombotic biomarkers (among which active VWF) in COPD and CKD. Sample sizes were rather small in both of our studies, in part due to larger than expected loss-to-follow-up and difficulties to include patients due to stringent exclusion criteria. This limitation also reflects the labour-intensive nature of these types of studies and the difficulty of organizing them in a peripheral (non-academic) medical centre. Nonetheless, due to the large changes in active VWF and other prothrombotic markers, we believe that future investigations into their relationship with the incidence of cardiovascular events in COPD and CKD patients are warranted.

As a consequence of our limited sample size, it was impossible to perform posthoc analyses on the effects of smoking and (acute) infection (in COPD patients). Inflammatory markers and the risk of CVD are known to have a dose-dependent relationship to smoking (cessation), ${ }^{112}$ and are also both increased following an acute infection. ${ }^{113}$ Our study was not powered to detect the effects of these parameters on our outcome measures, but this would be interesting to assess in future studies.

\section{Outcome measures}

With regard to outcome measures, our data suggest that in COPD patients, (active) VWF, PMCs, Mac-1 and TG should be candidate biomarkers in future studies. One specific point of attention when measuring PMCs in future studies is that we used CD14 positivity to define PMCs in our flow cytometric analysis. However, a recent study has shown that particularly (pro-inflammatory) intermediate and non-classical monocytes, characterized by high $\mathrm{CD} 16^{+++}$(and lower CD14 positivity), had increased aggregability to platelets compared to classical (phagocytic) $C D 14^{++} \mathrm{CD} 16^{-}$monocytes. ${ }^{114}$ Thus, future studies may consider also quantifying $\mathrm{CD}_{1} 6^{+/++}$monocyte-platelet aggregates. 
In CKD, based on our findings and existing literature, active VWF, VWF antigen, FVIII and ADAMTS13 (levels and activity, in blood and urine) require further investigation. Furthermore, besides changes in these coagulation parameters, it is important to quantify clinical outcomes, such as exacerbation rate (COPD), eGFR decline, kidney replacement therapy/haemodialysis (CKD), thrombotic events and death (both).

\section{Longer follow-up with more measurements}

Concerning follow up measurements, it would be advisable to include sequential time points over a longer period of follow-up. Such a design would allow for the detection of a larger number of cardiovascular outcome events and hence a stronger evidence base to draw conclusions on the causality of prothrombotic changes (i.e. during versus after an AE-COPD, and with the progression of CKD) and the subsequent risk of cardiovascular disease. Conceptually, there are at least two ways in which active VWF (and other prothrombotic biomarkers) may be related to thrombo-inflammatory conditions such as COPD and CKD: (1) as a manifestation/consequence of the disease itself, (2) as a causative factor in the disease aetiology/progression. In both situations, the level of the biomarker will be increased when you take a blood sample at a single time point, but sequential measurements are pivotal to unravel whether it is cause or consequence of the condition.

\section{Identification of targets to reduce thrombotic risk}

Once studies identify (a) biomarker(s) with a direct relationship to the clinical outcome (e.g. thrombotic risk, COPD exacerbation rate, renal endothelium damage), further studies should determine whether the biomarker can be useful for diagnostics and/or monitoring of disease progression, and in which patients. In addition, therapeutic strategies should then be considered. In general, the two modifiable therapeutic "targets" in COPD and CKD are the inflammation and coagulation pathways. As mentioned, there is extensive crosstalk between these processes, and it is very likely that the inflammatory and procoagulant responses in these and other inflammatory conditions are coincident and circular. Therefore, therapeutic interventions should be directed at breaking this continuous circle by treatment with anti-inflammatory and/or anti-thrombotic drugs. If active VWF would prove to have a causative role in these conditions, treatment options could focus on neutralizing the active VWF-platelet interaction (as discussed above) or for instance blocking IL-6-mediated inhibition of ADAMTS13, thereby enhancing its capacity to proteolyze active UL-VWF multimers.

However, it must also be acknowledged that COPD and CKD share many preventable and modifiable "lifestyle" risk factors, such as aging, a sedentary lifestyle, metabolic syndrome and of course smoking. ${ }^{115-118}$ Therefore, besides pharmacological strategies, 
reduction of the incidence of both conditions depends largely on primary prevention strategies (e.g. smoking cessation, exercise programs) targeting these risk factors. ${ }^{59}$

Altogether, gaining a better understanding of the underlying pathophysiological mechanisms in thrombo-inflammatory conditions such as COPD and CKD is imperative for evidence-based improvements in cardiovascular risk prediction and prevention. Future clinical outcome studies should provide guidance on which patients are at particularly high risk, and on the type and duration of thromboprophylaxis, while taking into account the (practically unavoidable) increased bleeding risk.

\section{Thrombolytics to resolve aTTP?}

As described in Chapter 9, data from animal and clinical studies suggest that plasmin may be an auxiliary VWF-cleaving protease. Numerous in vitro studies have shown that plasmin is indeed able to cleave shear-unfolded active VWF and even ADAMTS-13resistant VWF fibers. ${ }^{119}$ This role may be especially relevant in situations with decreased ADAMTS13 activity, as in TTP. ${ }^{120,121}$ Increased plasmin activity was indeed detected in patients with acute TTP ${ }^{120}$ but data that emerged after the publication of our review showed that these increased plasmin levels were not high enough to positively affect TTP outcomes, due to the presence of plasmin(ogen) inhibitors (i.e. $\alpha 2$-antiplasmin and PAl-1). ${ }^{122}$ By blocking these inhibitors, Tersteeg et al. showed that amplified endogenous plasmin activity did resolve aTTP signs in a TTP mouse model. This effect could be attributed to the increased proteolysis of VWF multimers. ${ }^{122}$ However, in patients, interference with physiological plasmin regulation by inhibition of PAI-1 and/or $\alpha 2$ antiplasmin is likely accompanied by a serious bleeding risk. An alternative strategy to achieve (temporary) supraphysiological plasmin activity would be exogenous activation of plasmin using thrombolytic therapy, such as streptokinase. Previous studies in mice demonstrated that low-dose streptokinase was sufficient to safely restore platelet counts. ${ }^{120}$ (Pre)clinical studies with streptokinase and other thrombolytic agents would be required to assess their efficacy and safety in TTP. This brings back the issue raised at the beginning of this chapter on the high costs of these (pre-)clinical studies while an alternative, caplacizumab, is approved and available.

\section{CONCLUSION}

In conclusion, the studies described in this thesis demonstrate that the SVWFA1 VHH, targeted against the $A 1$ domain of active VWF, has two potential applications: (1) as a possible therapeutic agent, by blocking (excessive, undesired) interactions between platelets and (2) as a capturing antibody (fragment) in an ELISA assay for the quan- 
tification of active VWF in plasma. Although more evidence for the clinical utility of active VWF as a biomarker needs to be obtained, our studies have laid the foundation for future (larger) clinical outcome studies by (1) validating the active VWF assay, (2) establishing reference ranges, (3) exploring the role of active VWF in several conditions of unbalanced haemostasis. 


\section{REFERENCES}

1. Hulstein JJ, de Groot PG, Silence K, Veyradier A, Fijnheer R, Lenting PJ. A novel nanobody that detects the gain-of-function phenotype of von Willebrand factor in ADAMTS13 deficiency and von Willebrand disease type 2B. Blood. 2005;106(9):3035-42.

2. Flood VH, Gill JC, Christopherson PA, Bellissimo DB, Friedman KD, Haberichter SL, et al. Critical von Willebrand factor $\mathrm{A} 1$ domain residues influence type VI collagen binding. J Thromb Haemost. 2012;10(7):1417-24.

3. Mohri H, Yoshioka A, Zimmerman TS, Ruggeri ZM. Isolation of the von Willebrand factor domain interacting with platelet glycoprotein $\mathrm{lb}$, heparin, and collagen and characterization of its three distinct functional sites. J Biol Chem. 1989;264(29):17361-7.

4. Morales LD, Martin C, Cruz MA. The interaction of von Willebrand factor-A1 domain with collagen: mutation G1324S (type $2 \mathrm{M}$ von Willebrand disease) impairs the conformational change in A1 domain induced by collagen. J Thromb Haemost. 2006;4(2):417-25.

5. Lankhof $H$, van Hoeij $M$, Schiphorst $M E$, Bracke $M, W u$ YP, ljsseldijk MJ, et al. A3 domain is essential for interaction of von Willebrand factor with collagen type III. Thromb Haemost. 1996;75(6):950-8.

6. Mazzucato M, Spessotto P, Masotti A, De Appollonia L, Cozzi MR, Yoshioka A, et al. Identification of domains responsible for von Willebrand factor type VI collagen interaction mediating platelet adhesion under high flow. J Biol Chem. 1999;274(5):3033-41.

7. Cruz MA, Yuan H, Lee JR, Wise RJ, Handin RI. Interaction of the von Willebrand factor (vWF) with collagen. Localization of the primary collagen-binding site by analysis of recombinant vWF A domain polypeptides. J Biol Chem. 1995;270(33):19668.

8. Springer TA. von Willebrand factor, Jedi knight of the bloodstream. Blood. 2014;124(9):141225.

9. Ju L, Chen Y, Zhou F, Lu H, Cruz MA, Zhu C. Von Willebrand factor-A1 domain binds platelet glycoprotein lbalpha in multiple states with distinctive force-dependent dissociation kinetics. Thromb Res. 2015;136(3):606-12.

10. Scully M, Cataland SR, Peyvandi F, Coppo P, Knobl P, Kremer Hovinga JA, et al. Caplacizumab Treatment for Acquired Thrombotic Thrombocytopenic Purpura. N Engl J Med. 2019;380(4):335-46.

11. Veyradier A. Von Willebrand Factor--A New Target for TTP Treatment? N Engl J Med. 2016;374(6):583-5.

12. Diener JL, Daniel Lagasse HA, Duerschmied D, Merhi Y, Tanguay JF, Hutabarat R, et al. Inhibition of von Willebrand factor-mediated platelet activation and thrombosis by the anti-von Willebrand factor A1-domain aptamer ARC1779. J Thromb Haemost. 2009;7(7):1155-62.

13. Gilbert JC, DeFeo-Fraulini T, Hutabarat RM, Horvath CJ, Merlino PG, Marsh HN, et al. First-inhuman evaluation of anti von Willebrand factor therapeutic aptamer ARC1779 in healthy volunteers. Circulation. 2007;116(23):2678-86.

14. Callewaert F, Roodt J, Ulrichts H, Stohr T, van Rensburg WJ, Lamprecht S, et al. Evaluation of efficacy and safety of the anti-VWF Nanobody ALX-0681 in a preclinical baboon model of acquired thrombotic thrombocytopenic purpura. Blood. 2012;120(17):3603-10.

15. Peyvandi F, Scully M, Kremer Hovinga JA, Cataland S, Knobl P, Wu H, et al. Caplacizumab for Acquired Thrombotic Thrombocytopenic Purpura. N Engl J Med. 2016;374(6):511-22.

16. Peyvandi F, Scully M, Kremer Hovinga JA, Knobl P, Cataland S, De Beuf K, et al. Caplacizumab reduces the frequency of major thromboembolic events, exacerbations and death 
in patients with acquired thrombotic thrombocytopenic purpura. J Thromb Haemost. 2017;15(7):1448-52.

17. Sargentini-Maier ML, De Decker P, Tersteeg C, Canvin J, Callewaert F, De Winter H. Clinical pharmacology of caplacizumab for the treatment of patients with acquired thrombotic thrombocytopenic purpura. Expert Rev Clin Pharmacol. 2019;12(6):537-45.

18. Castaman G, Linari S. Diagnosis and Treatment of von Willebrand Disease and Rare Bleeding Disorders. J Clin Med. 2017;6(4).

19. Eto K, Isshiki T, Yamamoto H, Takeshita S, Ochiai M, Yokoyama N, et al. AJvW-2, an anti-vWF monoclonal antibody, inhibits enhanced platelet aggregation induced by high shear stress in platelet-rich plasma from patients with acute coronary syndromes. Arterioscler Thromb Vasc Biol. 1999;19(4):877-82.

20. Kageyama S, Yamamoto H, Nagano M, Arisaka H, Kayahara T, Yoshimoto R. Anti-thrombotic effects and bleeding risk of AJvW-2, a monoclonal antibody against human von Willebrand factor. Br J Pharmacol. 1997;122(1):165-71.

21. Kageyama S, Yamamoto H, Nakazawa H, Yoshimoto R. Anti-human vWF monoclonal antibody, AJvW-2 Fab, inhibits repetitive coronary artery thrombosis without bleeding time prolongation in dogs. Thromb Res. 2001;101(5):395-404.

22. Kageyama S, Yamamoto H, Yoshimoto R. Anti-human von willebrand factor monoclonal antibody AJvW-2 prevents thrombus deposition and neointima formation after balloon injury in guinea pigs. Arterioscler Thromb Vasc Biol. 2000;20(10):2303-8.

23. Kageyama S, Yamamoto H, Nakazawa H, Matsushita J, Kouyama T, Gonsho A, et al. Pharmacokinetics and pharmacodynamics of AJW200, a humanized monoclonal antibody to von Willebrand factor, in monkeys. Arterioscler Thromb Vasc Biol. 2002;22(1):187-92.

24. Kageyama S, Matsushita J, Yamamoto H. Effect of a humanized monoclonal antibody to von Willebrand factor in a canine model of coronary arterial thrombosis. Eur J Pharmacol. 2002;443(1-3):143-9.

25. Jilma-Stohlawetz P, Gilbert JC, Gorczyca ME, Knobl P, Jilma B. A dose ranging phase I/II trial of the von Willebrand factor inhibiting aptamer ARC1779 in patients with congenital thrombotic thrombocytopenic purpura. Thromb Haemost. 2011;106(3):539-47.

26. Cataland SR, Peyvandi F, Mannucci PM, Lammle B, Kremer Hovinga JA, Machin SJ, et al. Initial experience from a double-blind, placebo-controlled, clinical outcome study of ARC1779 in patients with thrombotic thrombocytopenic purpura. Am J Hematol. 2012;87(4):430-2.

27. Jilma-Stohlawetz P, Gorczyca ME, Jilma B, Siller-Matula J, Gilbert JC, Knobl P. Inhibition of von Willebrand factor by ARC1779 in patients with acute thrombotic thrombocytopenic purpura. Thromb Haemost. 2011;105(3):545-52.

28. Markus HS, McCollum C, Imray C, Goulder MA, Gilbert J, King A. The von Willebrand inhibitor ARC1779 reduces cerebral embolization after carotid endarterectomy: a randomized trial. Stroke. 2011;42(8):2149-53.

29. Jilma-Stohlawetz P, Knobl P, Gilbert JC, Jilma B. The anti-von Willebrand factor aptamer ARC1779 increases von Willebrand factor levels and platelet counts in patients with type 2B von Willebrand disease. Thromb Haemost. 2012;108(2):284-90.

30. Siller-Matula JM, Merhi Y, Tanguay JF, Duerschmied D, Wagner DD, McGinness KE, et at. ARC15105 is a potent antagonist of von Willebrand factor mediated platelet activation and adhesion. Arterioscler Thromb Vasc Biol. 2012;32(4):902-9. 
31. Bartunek J, Barbato E, Heyndrickx G, Vanderheyden M, Wijns W, Holz JB. Novel antiplatelet agents: ALX-0081, a Nanobody directed towards von Willebrand factor. J Cardiovasc Transl Res. 2013;6(3):355-63.

32. van Loon JE, de Jaegere PP, Ulrichts $\mathrm{H}$, van Vliet $\mathrm{HH}$, de Maat MP, de Groot PG, et al. The in vitro effect of the new antithrombotic drug candidate ALX-0081 on blood samples of patients undergoing percutaneous coronary intervention. Thromb Haemost. 2011;106(1):165-71.

33. Zhu S, Gilbert JC, Hatala P, Harvey W, Liang Z, Gao S, et al. The Development and Characterization of a Long Acting Anti-thrombotic von Willebrand Factor (VWF) Aptamer. J Thromb Haemost. 2020.

34. Tersteeg C, Roodt J, Van Rensburg WJ, Dekimpe C, Vandeputte N, Pareyn I, et al. N-acetylcysteine in preclinical mouse and baboon models of thrombotic thrombocytopenic purpura. Blood. 2017;129(8):1030-8.

35. Duttaroy A, Kanakaraj P, Osborn BL, Schneider H, Pickeral OK, Chen C, et al. Development of a long-acting insulin analog using albumin fusion technology. Diabetes. 2005;54(1):251-8.

36. Siller-Matula JM, Plasenzotti R, Spiel A, Quehenberger P, Jilma B. Interspecies differences in coagulation profile. Thromb Haemost. 2008;100(3):397-404.

37. Gershoni JM, Roitburd-Berman A, Siman-Tov DD, Tarnovitski Freund N, Weiss Y. Epitope mapping: the first step in developing epitope-based vaccines. BioDrugs. 2007;21(3):145-56.

38. Wei H, Mo J, Tao L, Russell RJ, Tymiak AA, Chen G, et al. Hydrogen/deuterium exchange mass spectrometry for probing higher order structure of protein therapeutics: methodology and applications. Drug Discov Today. 2014;19(1):95-102.

39. Coales SJ, Tuske SJ, Tomasso JC, Hamuro Y. Epitope mapping by amide hydrogen/deuterium exchange coupled with immobilization of antibody, on-line proteolysis, liquid chromatography and mass spectrometry. Rapid Commun Mass Spectrom. 2009;23(5):639-47.

40. Deng W, Wang Y, Druzak SA, Healey JF, Syed AK, Lollar P, et al. A discontinuous autoinhibitory module masks the A1 domain of von Willebrand factor. J Thromb Haemost. 2017;15(9):186777.

41. Ruggeri ZM. The role of von Willebrand factor in thrombus formation. Thromb Res. 2007;120 Suppl 1:S5-9.

42. Jones G, Barker A. Reference intervals. Clin Biochem Rev. 2008;29 Suppl 1:S93-7.

43. van der Vorm LN, Li L, Huskens D, Chayoua W, Kelchtermans H, de Groot PG, et al. Analytical characterization and reference interval of an enzyme-linked immunosorbent assay for active von Willebrand factor. PLoS One. 2019;14(2):e0211961.

44. Shiroma EJ, Lee IM. Physical activity and cardiovascular health: lessons learned from epidemiological studies across age, gender, and race/ethnicity. Circulation. 2010;122(7):743-52.

45. Swift DL, Lavie CJ, Johannsen NM, Arena R, Earnest CP, O'Keefe JH, et al. Physical activity, cardiorespiratory fitness, and exercise training in primary and secondary coronary prevention. Circ J. 2013:77(2):281-92.

46. Albano AJ, Thompson PD, Kapur NK. Acute coronary thrombosis in Boston marathon runners. N Engl J Med. 2012;366(2):184-5.

47. Tak S, Tak S. Lower extremity deep vein thrombosis after heavy exertion. BMJ Case Rep. 2013;2013.

48. Schmied C, Borjesson M. Sudden cardiac death in athletes. J Intern Med. 2014;275(2):93103.

49. Parto P, O'Keefe JH, Lavie CJ. The Exercise Rehabilitation Paradox: Less May Be More? Ochsner J. 2016;16(3):297-303. 
50. Posthuma JJ, van der Meijden PE, Ten Cate H, Spronk HM. Short- and Long-term exercise induced alterations in haemostasis: a review of the literature. Blood Rev. 2015;29(3):171-8.

51. Kicken $\mathrm{CH}$, Miszta A, Kelchtermans H, De Laat B. Hemostasis during Extreme Exertion. Semin Thromb Hemost. 2018;44(7):640-50.

52. van Loon JE, Sonneveld MA, Praet SF, de Maat MP, Leebeek FW. Performance related factors are the main determinants of the von Willebrand factor response to exhaustive physical exercise. PLoS One. 2014;9(3):e91687.

53. van der Vorm LN, Huskens D, Kicken CH, Remijn JA, Roest M, de Laat B, et al. Effects of Repeated Bouts of Exercise on the Hemostatic System. Semin Thromb Hemost. 2018;44(8):710-22.

54. Huskens D, Roest M, Remijn JA, Konings J, Kremers RM, Bloemen S, et al. Strenuous exercise induces a hyperreactive rebalanced haemostatic state that is more pronounced in men. Thromb Haemost. 2016;115(6):1109-19.

55. Kiouptsi K, Gambaryan S, Walter E, Walter U, Jurk K, Reinhardt C. Hypoxia impairs agonist-induced integrin alphallbbeta3 activation and platelet aggregation. Sci Rep. 2017;7(1):7621.

56. Wang JS, Chang YL, Chen YC, Tsai HH, Fu TC. Effects of normoxic and hypoxic exercise regimens on monocyte-mediated thrombin generation in sedentary men. Clin Sci (Lond). 2015;129(4):363-74.

57. Townsend NE, Gore CJ, Ebert TR, Martin DT, Hahn AG, Chow CM. Ventilatory acclimatisation is beneficial for high-intensity exercise at altitude in elite cyclists. Eur J Sport Sci. 2016;16(8):895-902.

58. Ploszczyca K, Langfort J, Czuba M. The Effects of Altitude Training on Erythropoietic Response and Hematological Variables in Adult Athletes: A Narrative Review. Front Physiol. 2018;9:375.

59. 2019 Global Strategy for the Diagnosis, Management, and Prevention of COPD. Global Initiative for Chronic Obstructive Lung Disease (GOLD) 2018 [Available from: https://goldcopd. org/.

60. Garvey C, Bayles MP, Hamm LF, Hill K, Holland A, Limberg TM, et al. Pulmonary Rehabilitation Exercise Prescription in Chronic Obstructive Pulmonary Disease: Review of Selected Guidelines: an official statement from the american association of cardiovascular and pulmonary rehabilitation. . J Cardiopulm Rehabil Prev. 2016;36(2):75-83.

61. van Helvoort HA, van de Pol MH, Heijdra YF, Dekhuijzen PN. Systemic inflammatory response to exhaustive exercise in patients with chronic obstructive pulmonary disease. Respir Med. 2005;99(12):1555-67.

62. Rochester CL. Exercise training in chronic obstructive pulmonary disease. J Rehabil Res Dev. 2003;40(5 Suppl 2):59-80.

63. Bar-Or D, Carrick MM, Mains CW, Rael LT, Slone D, Brody EN. Sepsis, oxidative stress, and hypoxia: Are there clues to better treatment? Redox Rep. 2015;20(5):193-7.

64. van Helvoort HA, Heijdra YF, Heunks LM, Meijer PL, Ruitenbeek W, Thijs HM, et al. Supplemental oxygen prevents exercise-induced oxidative stress in muscle-wasted patients with chronic obstructive pulmonary disease. Am J Respir Crit Care Med. 2006;173(10):1122-9.

65. Kestin AS, Ellis PA, Barnard MR, Errichetti A, Rosner BA, Michelson AD. Effect of strenuous exercise on platelet activation state and reactivity. Circulation. 1993;88(4 Pt 1):1502-11.

66. Anthonisen NR, Connett JE, Enright PL, Manfreda J, Lung Health Study Research G. Hospitalizations and mortality in the Lung Health Study. Am J Respir Crit Care Med. 2002;166(3):3339. 
67. Vestbo J, Hurd SS, Agusti AG, Jones PW, Vogelmeier C, Anzueto A, et al. Global strategy for the diagnosis, management, and prevention of chronic obstructive pulmonary disease: GOLD executive summary. Am J Respir Crit Care Med. 2013;187(4):347-65.

68. Donaldson GC, Hurst JR, Smith CJ, Hubbard RB, Wedzicha JA. Increased risk of myocardial infarction and stroke following exacerbation of COPD. Chest. 2010;137(5):1091-7.

69. Maclay JD, McAllister DA, Macnee W. Cardiovascular risk in chronic obstructive pulmonary disease. Respirology. 2007;12(5):634-41.

70. Lozano R, Naghavi M, Foreman K, Lim S, Shibuya K, Aboyans V, et al. Global and regional mortality from 235 causes of death for 20 age groups in 1990 and 2010: a systematic analysis for the Global Burden of Disease Study 2010. Lancet. 2012;380(9859):2095-128.

71. Rothnie KJ, Yan R, Smeeth L, Quint JK. Risk of myocardial infarction (MI) and death following $\mathrm{MI}$ in people with chronic obstructive pulmonary disease (COPD): a systematic review and meta-analysis. BMJ Open. 2015;5(9):e007824.

72. Davi G, Patrono C. Platelet activation and atherothrombosis. N Engl J Med. 2007;357(24):2482-94.

73. Maclay JD, McAllister DA, Johnston S, Raftis J, McGuinnes C, Deans A, et al. Increased platelet activation in patients with stable and acute exacerbation of COPD. Thorax. 2011;66(9):76974.

74. Michelson AD, Barnard MR, Krueger LA, Valeri CR, Furman MI. Circulating monocyte-platelet aggregates are a more sensitive marker of in vivo platelet activation than platelet surface P-selectin: studies in baboons, human coronary intervention, and human acute myocardial infarction. Circulation. 2001;104(13):1533-7.

75. Michelson AD, Barnard MR, Hechtman HB, MacGregor H, Connolly RJ, Loscalzo J, et al. In vivo tracking of platelets: circulating degranulated platelets rapidly lose surface P-selectin but continue to circulate and function. Proc Natl Acad Sci U S A. 1996;93(21):11877-82.

76. Simon DI, Chen Z, Xu H, Li CO, Dong J, McIntire LV, et al. Platelet glycoprotein ibalpha is a counterreceptor for the leukocyte integrin Mac-1 (CD11b/CD18). J Exp Med. 2000;192(2):193204.

77. Neumann FJ, Ott I, Marx N, Luther T, Kenngott S, Gawaz M, et al. Effect of human recombinant interleukin-6 and interleukin-8 on monocyte procoagulant activity. Arterioscler Thromb Vasc Biol. 1997;17(12):3399-405.

78. Altieri DC, Morrissey JH, Edgington TS. Adhesive receptor Mac-1 coordinates the activation of factor $X$ on stimulated cells of monocytic and myeloid differentiation: an alternative initiation of the coagulation protease cascade. Proc Natl Acad Sci U S A. 1988;85(20):7462-6.

79. Corken A, Russell S, Dent J, Post SR, Ware J. Platelet glycoprotein Ib-IX as a regulator of systemic inflammation. Arterioscler Thromb Vasc Biol. 2014;34(5):996-1001.

80. Polosa R, Malerba M, Cacciola RR, Morjaria JB, Maugeri C, Prosperini G, et al. Effect of acute exacerbations on circulating endothelial, clotting and fibrinolytic markers in COPD patients. Intern Emerg Med. 2013;8(7):567-74.

81. Polatli M, Cakir A, Cildag O, Bolaman AZ, Yenisey C, Yenicerioglu Y. Microalbuminuria, von Willebrand factor and fibrinogen levels as markers of the severity in COPD exacerbation. J Thromb Thrombolysis. 2008;26(2):97-102.

82. Bernardo A, Ball C, Nolasco L, Moake JF, Dong JF. Effects of inflammatory cytokines on the release and cleavage of the endothelial cell-derived ultralarge von Willebrand factor multimers under flow. Blood. 2004;104(1):100-6. 
83. Versteeg HH, Heemskerk JW, Levi M, Reitsma PH. New fundamentals in hemostasis. Physiol Rev. 2013;93(1):327-58.

84. Celli BR, Locantore N, Yates J, Tal-Singer R, Miller BE, Bakke P, et al. Inflammatory biomarkers improve clinical prediction of mortality in chronic obstructive pulmonary disease. Am J Respir Crit Care Med. 2012;185(10):1065-72.

85. Thomsen M, Dahl M, Lange P, Vestbo J, Nordestgaard BG. Inflammatory biomarkers and comorbidities in chronic obstructive pulmonary disease. Am J Respir Crit Care Med. 2012;186(10):982-8.

86. Young RP, Hopkins RJ. Update on the potential role of statins in chronic obstructive pulmonary disease and its co-morbidities. Expert Rev Respir Med. 2013;7(5):533-44.

87. Shoji T, Koyama H, Fukumoto S, Maeno T, Yokoyama H, Shinohara K, et al. Platelet-monocyte aggregates are independently associated with occurrence of carotid plaques in type 2 diabetic patients. J Atheroscler Thromb. 2005;12(6):344-52.

88. Harrison MT, Short P, Williamson PA, Singanayagam A, Chalmers JD, Schembri S. Thrombocytosis is associated with increased short and long term mortality after exacerbation of chronic obstructive pulmonary disease: a role for antiplatelet therapy? Thorax. 2014;69(7):609-15.

89. Braun OO, Johnell M, Varenhorst C, James S, Brandt JT, Jakubowski JA, et al. Greater reduction of platelet activation markers and platelet-monocyte aggregates by prasugrel compared to clopidogrel in stable coronary artery disease. Thromb Haemost. 2008;100(4):626-33.

90. Frelinger AL, 3rd, Jakubowski JA, Li Y, Barnard MR, Linden MD, Tarnow I, et al. The active metabolite of prasugrel inhibits adenosine diphosphate- and collagen-stimulated platelet procoagulant activities. J Thromb Haemost. 2008;6(2):359-65.

91. Kunadian V, Wilson N, Stocken DD, Ali H, McColl E, Burns G, et al. Antiplatelet therapy in the primary prevention of cardiovascular disease in patients with chronic obstructive pulmonary disease: a randomised controlled proof-of-concept trial. ERJ Open Res. 2019;5(3).

92. Schunemann HJ, Cushman M, Burnett AE, Kahn SR, Beyer-Westendorf J, Spencer FA, et al. American Society of Hematology 2018 guidelines for management of venous thromboembolism: prophylaxis for hospitalized and nonhospitalized medical patients. Blood Adv. 2018;2(22):3198-225.

93. Ludwig RJ. Therapeutic use of heparin beyond anticoagulation. Curr Drug Discov Technol. 2009;6(4):281-9.

94. Oian Y, Xie H, Tian R, Yu K, Wang R. Efficacy of low molecular weight heparin in patients with acute exacerbation of chronic obstructive pulmonary disease receiving ventilatory support. COPD. 2014;11(2):171-6.

95. Yang $M, X u Y$, Chen $H, X u Z$, Luo F. Benefits and risks of low molecular weight heparin in patients with acute exacerbation of chronic obstructive pulmonary disease: a meta-analysis of randomized controlled trials. Inflammopharmacology. 2019.

96. Levey AS, Becker C, Inker LA. Glomerular filtration rate and albuminuria for detection and staging of acute and chronic kidney disease in adults: a systematic review. JAMA. 2015;313(8):837-46.

97. Hill NR, Fatoba ST, Oke JL, Hirst JA, O'Callaghan CA, Lasserson DS, et al. Global Prevalence of Chronic Kidney Disease - A Systematic Review and Meta-Analysis. PLoS One. 2016;11(7):e0158765.

98. Go AS, Chertow GM, Fan D, McCulloch CE, Hsu CY. Chronic kidney disease and the risks of death, cardiovascular events, and hospitalization. N Engl J Med. 2004;351(13):1296-305. 
99. Cheung KL, Zakai NA, Folsom AR, Kurella Tamura M, Peralta CA, Judd SE, et al. Measures of Kidney Disease and the Risk of Venous Thromboembolism in the REGARDS (Reasons for Geographic and Racial Differences in Stroke) Study. Am J Kidney Dis. 2017;70(2):182-90.

100. Mahmoodi BK, Gansevoort RT, Naess IA, Lutsey PL, Braekkan SK, Veeger NJ, et al. Association of mild to moderate chronic kidney disease with venous thromboembolism: pooled analysis of five prospective general population cohorts. Circulation. 2012;126(16):1964-71.

101. Mihai S, Codrici E, Popescu ID, Enciu AM, Albulescu L, Necula LG, et al. Inflammation-Related Mechanisms in Chronic Kidney Disease Prediction, Progression, and Outcome. J Immunol Res. 2018;2018:2180373.

102. Sprague AH, Khalil RA. Inflammatory cytokines in vascular dysfunction and vascular disease. Biochem Pharmacol. 2009;78(6):539-52.

103. Dunn A, Lo V, Donnelly S. The role of the kidney in blood volume regulation: the kidney as a regulator of the hematocrit. Am J Med Sci. 2007;334(1):65-71.

104. Huang MJ, Wei RB, Wang Y, Su TY, Di P, Li QP, et al. Blood coagulation system in patients with chronic kidney disease: a prospective observational study. BMJ Open. 2017;7(5):e014294.

105. Pequeriaux NC, Fijnheer R, Gemen EF, Barendrecht AD, Dekker FW, Krediet RT, et al. Plasma concentration of von Willebrand factor predicts mortality in patients on chronic renal replacement therapy. Nephrol Dial Transplant. 2012;27(6):2452-7.

106. Shen L, Lu G, Dong N, Jiang L, Ma Z, Ruan C. Von Willebrand factor, ADAMTS13 activity, TNF-alpha and their relationships in patients with chronic kidney disease. Exp Ther Med. 2012;3(3):530-4.

107. Jager A, van Hinsbergh VW, Kostense PJ, Emeis JJ, Yudkin JS, Nijpels G, et al. von Willebrand factor, C-reactive protein, and 5-year mortality in diabetic and nondiabetic subjects: the Hoorn Study. Arterioscler Thromb Vasc Biol. 1999;19(12):3071-8.

108. Ocak G, Vossen CY, Lijfering WM, Verduijn M, Dekker FW, Rosendaal FR, et al. Role of hemostatic factors on the risk of venous thrombosis in people with impaired kidney function. Circulation. 2014;129(6):683-91.

109. Matsushita K, Coresh J, Sang Y, Chalmers J, Fox C, Guallar E, et al. Estimated glomerular filtration rate and albuminuria for prediction of cardiovascular outcomes: a collaborative meta-analysis of individual participant data. Lancet Diabetes Endocrinol. 2015;3(7):51425.

110. Casonato A, Pontara E, Morpurgo M, Sartorello F, De Groot PG, Cattini MG, et al. Higher and lower active circulating VWF levels: different facets of von Willebrand disease. $\mathrm{Br}]$ Haematol. 2015;171(5):845-53.

111. Groot E, de Groot PG, Fijnheer R, Lenting PJ. The presence of active von Willebrand factor under various pathological conditions. Curr Opin Hematol. 2007;14(3):284-9.

112. Bakhru A, Erlinger TP. Smoking cessation and cardiovascular disease risk factors: results from the Third National Health and Nutrition Examination Survey. PLoS Med. 2005;2(6):e160.

113. Cowan LT, Lutsey PL, Pankow JS, Matsushita K, Ishigami J, Lakshminarayan K. Inpatient and Outpatient Infection as a Trigger of Cardiovascular Disease: The ARIC Study. J Am Heart Assoc. 2018;7(22):e009683.

114. Allen N, Barrett TJ, Guo Y, Nardi M, Ramkhelawon B, Rockman CB, et al. Circulating monocyte-platelet aggregates are a robust marker of platelet activity in cardiovascular disease. Atherosclerosis. 2019;282:11-8. 
115. Martens RJH, van der Berg JD, Stehouwer CDA, Henry RMA, Bosma H, Dagnelie PC, et al. Amount and pattern of physical activity and sedentary behavior are associated with kidney function and kidney damage: The Maastricht Study. PLoS One. 2018;13(4):e0195306.

116. Schneider LP, Furlanetto KC, Rodrigues A, Lopes JR, Hernandes NA, Pitta F. Sedentary Behaviour and Physical Inactivity in Patients with Chronic Obstructive Pulmonary Disease: Two Sides of the Same Coin? COPD. 2018;15(5):432-8.

117. Thomas G, Sehgal AR, Kashyap SR, Srinivas TR, Kirwan JP, Navaneethan SD. Metabolic syndrome and kidney disease: a systematic review and meta-analysis. Clin J Am Soc Nephrol. 2011;6(10):2364-73.

118. Uzokov J, Alyavi A, Karimov B. Prevalence of metabolic syndrome in patients with chronic obstructive pulmonary disease. European Respiratory Journal. 2016;48(suppl 60):PA3776.

119. Wohner N, Kovacs A, Machovich R, Kolev K. Modulation of the von Willebrand factordependent platelet adhesion through alternative proteolytic pathways. Thromb Res. 2012;129(4):e41-6.

120. Tersteeg C, de Maat S, De Meyer SF, Smeets MW, Barendrecht AD, Roest M, et al. Plasmin cleavage of von Willebrand factor as an emergency bypass for ADAMTS13 deficiency in thrombotic microangiopathy. Circulation. 2014;129(12):1320-31.

121. van der Vorm LN, Remijn JA, De Laat B, Huskens D. Effects of Plasmin on von Willebrand Factor and Platelets: A Narrative Review. TH Open. 2018;2(2):e218-e28.

122. Tersteeg C, Joly BS, Gils A, Lijnen R, Deckmyn H, Declerck PJ, et al. Amplified endogenous plasmin activity resolves acute thrombotic thrombocytopenic purpura in mice. J Thromb Haemost. 2017;15(12):2432-42. 



$$
\text { Chapter } 11
$$


Nederlandse samenvatting 



\section{NEDERLANDSE SAMENVATTING}

In dit proefschrift beschrijven we onze studies met als doel om meer inzicht te krijgen in de in vitro en in vivo condities die bijdragen aan activatie van von Willebrand Factor (VWF) en binding van actief VWF aan bloedplaatjes. Hiertoe hebben we zowel mechanistische laboratorium studies als klinische studies in gezonde individuen en verschillende patiënten populaties uitgevoerd.

Hoofdstuk 1 van dit proefschrift vormt de algemene introductie, bestaande uit achtergrondinformatie over de bloedstolling en de rationale achter dit proefschrift. Naast de fysiologische hemostase worden afwijkingen in de hoeveelheid of functie van (actief) VWF besproken die de balans van de bloedstolling naar de bloedings- (bijvoorbeeld de ziekte van von Willebrand) of de trombotische kant (arteriële/veneuze trombose) kunnen laten doorslaan.

Gezien de grote hemostatische potentie van actief VWF is het van cruciaal belang om te begrijpen welke factoren bijdragen aan veranderingen in de hoeveelheid circulerend actief VWF en de interactie met bloedplaatjes. Dit is dan ook het overkoepelende doel van de studies in dit proefschrift. De belangrijkste "tool" waarmee we actief VWF en de interactie met bloedplaatjes hebben onderzocht is een antilichaam fragment bestaande uit een enkele zware keten (afgekort VHH). Deze VHH, genaamd S-VWFA1, is gebruikt in alle experimenten beschreven in dit proefschrift en bindt specifiek aan het A1 domein van actief VWF. Hieronder volgt een samenvatting van de uitgevoerde studies en de belangrijkste bevindingen.

\section{Deel I. Detectie van actief VWF door een VHH: mechanismen en assay validatie}

Aangezien bloedplaatjes net als onze VHH binden aan een epitoop in het VWF A1 domein, verwachtten we dat de VHH de interactie tussen bloedplaatjes en actief VWF zou kunnen blokkeren. In hoofdstuk 2 van dit proefschrift beschrijven we experimenten met als doel het in kaart brengen van het effect van S-VWFA1 VHH op de interactie tussen bloedplaatjes en VWF. In een flowcytometrische test en in licht transmissie aggregometrie kon S-VWFA1 de binding van bloedplaatjes aan VWF geactiveerd met ristocetine (i.e. een antibioticum dat VWF ontvouwt) en aan actief VWF met een type 2B mutatie volledig blokkeren. Echter, in een perfusie assay en in de PFA-200®, waarbij bloedplaatjes en VWF op hoge snelheid over een oppervlak met collageen stromen, kon S-VWFA1 de adhesie van plaatjes niet remmen, of pas bij heel hoge concentraties ( $>50$ $\mu \mathrm{g} / \mathrm{mL}$ ). GPIb binding aan collageen-gebonden VWF in een statische assay bleef ook onaangedaan door S-VWFA1. Uit deze resultaten concluderen we dat S-VWFA1 alleen 
bloedplaatjes binding aan actief VWF in oplossing blokkeert, maar niet aan collageengebonden actief VWF.

Neutralisatie van de interactie tussen bloedplaatjes en VWF zou een aantrekkelijke behandelingsstrategie zijn bij aandoeningen die gepaard gaan met verhoogde hoeveelheden circulerend actief VWF. Om te bepalen of een bepaalde ziekte geassocieerd is met verhoogde concentraties actief VWF dient eerst te worden onderzocht welke waarden men zou verwachten in een gezonde populatie. In hoofdstuk 3 rapporteren we de technische validatie van een ELISA waarin S-VWFA1 gebruikt wordt om actief VWF te "vangen". ${ }^{1}$ Deze assay was specifiek voor actief VWF, reproduceerbaar (intra- en interassay CVs tussen de $\mathbf{2 . 4 - 7 . 2 \% ~ e n ~ 4 . 1 - 9 . 4 \% ) ~ e n ~ l i n e a i r ~ i n ~ d e ~ r a n g e ~ v a n ~} \mathbf{9 0 . 1 - 4 2 4 . 5 \%}$ (t.o.v. normaal gepoold plasma). Daarnaast hebben we met deze assay actief VWF bepaald in 120 gezonde individuen. Deze waardes voor actief VWF correleerden significant met andere, gevestigde VWF assays en het referentie interval werd vastgesteld op 91.6-154.8\%. De gevalideerde assay is vervolgens gebruikt om actief VWF te meten in gezonde individuen die intensief sporten en bij verschillende patiënten populaties.

\section{Deel II. Effecten van (herhaalde) intensieve lichamelijke inspanning op (actief) VWF}

Aan bovenstaand referentie interval is te zien dat de fractie actief VWF in de circulatie varieert tussen gezonde individuen. Eén van de niet-erfelijke factoren die het vrijkomen van VWF uit het endotheel stimuleert is intensieve lichamelijke inspanning. Het is algemeen bekend dat regelmatig bewegen het risico op hart- en vaatziekten verlaagt. Echter, meer en intensievere lichaamsbeweging is niet altijd beter en kan juist ook veneuze en arteriële trombose veroorzaken. Dit wordt ook wel de "exercise paradox" genoemd.

Sporten beïnvloedt de bloedstolling op verschillende manieren. Zo is bekend dat vooral in ongetrainde mensen acute hevige inspanning een verhoogde stollingsneiging veroorzaakt. Herhaaldelijk sporten (trainen) zou de hemostatische respons wellicht kunnen conditioneren, maar daarover is veel minder bekend. Hoofdstuk 4 geeft daarom een overzicht van de effecten van herhaald intensief sporten op de bloedstolling, gebaseerd op recente literatuur. Daarnaast presenteren we in dit hoofdstuk data van een pilot studie waarin we de effecten van herhaald fietsen (80 km op zeeniveau, op submaximale intensiteit) op de bloedstolling hebben bestudeerd. ${ }^{2}$ Het was reeds bekend dat endotheel activatie cruciaal is voor de toegenomen stollingsneiging als gevolg van acute intensieve lichamelijke inspanning. Echter, we vonden dat de toename van FVIII, VWF antigeen, propeptide en actief VWF kleiner werd op elk van de drie opvolgende dagen dat onze proefpersonen hetzelfde parcours fietsten. Dit effect suggereert ofwel 
adaptatie van de respons van het endotheel op de inspanning of uitputting van de VWF voorraad in de endotheelcellen. De verhoogde FVIII concentratie resulteerde in een hogere piek trombine generatie. De bloedplaatjes activatie daarentegen, gemeten als P-selectine expressie na stimulatie met verschillende agonisten, nam juist af, en compenseert daarmee deels voor de endotheel-geïnduceerde protrombotische staat. Deze verlaagde bloedplaatjes activatie herstelde niet naar het originele niveau op de volgende dag voorafgaand aan het fietsen, duidend op uitputting van de respons van bloedplaatjes op herhaalde intensieve inspanning.

In een vervolgstudie, beschreven in hoofdstuk $\mathbf{5}$ werd een extra variabele geïntroduceerd, namelijk hypoxie. ${ }^{3}$ Het is bekend dat trainen op grote hoogte, waar de zuurstofspanning lager is, leidt tot een verhoogde concentratie hemoglobine in het bloed, waardoor het zuurstoftransport naar o.a. de spieren verbetert. Echter, hypoxie induceert ook een verhoogde stollingsneiging, o.a. via een verhoogde FVIII concentratie. Aangezien zowel hypoxie als inspanning de stolling lijken te activeren, zou men verwachten dat inspanning op grote hoogte een wederzijds versterkend effect zou hebben. Het omgekeerde lijkt het geval in meerdere studies die beschrijven dat hypoxie de protrombotische effecten van inspanning juist dempt. In deze pilot studie werd onderzocht of dit ook geldt voor herhaalde inspanning, zoals optreedt bij training op grote hoogte en bij bergbeklimmers. Hiervoor voerden zes, lichamelijk fitte mannen in totaal 4 inspanningstesten (fietsen) van 2 uur uit: 1 keer op zeeniveau en 3 keer op achtereenvolgende dagen op 3,375 m hoogte. Naast markers voor endotheel activatie (actief VWF, VWF antigeen en propeptide) werden plasmatische stolling (trombine generatie), bloedplaatjesactiviteit en stolselafbraak gemeten. De metingen op zeeniveau bevestigden onze resultaten van de eerste fietsstudie, namelijk verhoogd actief VWF, VWF antigeen, FVIII en daardoor ook een verhoogde trombine generatie piek na het fietsen. Weer was de degranulatie (P-selectine expressie) van bloedplaatjes juist verlaagd na de inspanningstest. Op 3,375 m hoogte was het aggregatie potentiaal van de bloedplaatjes ook lager. Al met al kunnen we concluderen dat intensieve lichamelijke inspanning een verhoogde stollingsneiging induceert die met name wordt gemedieerd door het endotheel (toename in (actief) VWF en FVIII). Na herhaalde inspanning wordt deze respons van het endotheel kleiner. Hypoxie dempt de protrombotische staat verder door het verminderen van de plaatjesactivatie.

De twee bovenstaande pilot studies hebben een aantal beperkingen, met name het kleine aantal deelnemers, bovendien allemaal mannen, waardoor de resultaten voorzichtig moeten worden geïnterpreteerd. Een grotere follow-up studie is nodig om klinisch relevante veranderingen te identificeren en om te onderzoeken of onze bevindingen ook geldig zijn in vrouwen. 


\section{Deel III. Actief VWF in aandoeningen met ongebalanceerde bloedstolling}

Na het bestuderen van actief VWF in gezonde individuen in de eerste twee delen van dit proefschrift, hebben we gekeken naar de rol van actief VWF in een drietal aandoeningen die geassocieerd zijn met een trombotische- of bloedingsneiging.

Hoofdstuk 6 rapporteert een prospectieve observationele cohort studie in patiënten met chronische obstructieve longziekte (COPD). Deze patiënten hebben een verhoogd risico op ischemische hart- en vaatziekten, met name tijdens en kort na een exacerbatie (i.e. plotselinge verergering van de symptomen die langer dan 2 dagen aanhouden). Exacerbaties worden meestal veroorzaakt door een infectie en gaan gepaard met verhoogde systemische ontsteking. Ontsteking is bovendien sterk gelinkt aan bloedstolling. Het doel van deze studie was daarom om de effecten van een exacerbatie van COPD op de bloedplaatjes, het endotheel en de plasmatische stolling in kaart te brengen, en deze te relateren aan de mate van ontsteking. Bij 52 patiënten werd bloed verzameld tijdens de exacerbatie, 3 dagen erna (tijdens behandeling in het ziekenhuis), en bij 32 van deze patiënten ook bij herstel ( 8 weken na ontslag). Vergeleken met herstel werd er tijdens exacerbatie een stijging in het aantal plaatjes-monocyten complexen (PMCs) vastgesteld, maar geen verhoogde P-selectine expressie of activatie van de fibrinogeenreceptor op plaatjes. Bovendien was er tijdens een exacerbatie sprake van chronische endotheel activatie die resulteert in verhoogde VWF antigeen en activiteit en in stijging van actief VWF, maar in stabiele VWF propeptide. Ten derde was de trombine generatie (piek, ETP en snelheid) verhoogd tijdens een exacerbatie vergeleken met herstel. PMC vorming, (actief) VWF en trombine generatie waren allen positief geassocieerd met de ontstekingsmarker CRP. Deze bevindingen dragen bij aan het begrip van de protrombotische staat van COPD patiënten tijdens en na een exacerbatie. Ze vormen zo de basis voor toekomstige studies naar markers die het risico van deze patiënten op een cardiovasculair event kunnen voorspellen en daarmee richting kunnen geven aan het beleid voor trombose profylaxe.

Een andere ziekte die geassocieerd is met zowel systemische ontsteking als een verhoogd trombotisch risico is chronische nierinsufficiëntie/nierfalen. Een eerdere studie vond endotheel dysfunctie in deze patiënten, gekenmerkt door toename in VWF antigeen en FVIII activiteit. ${ }^{4}$ Wij waren geïnteresseerd in de hoeveelheid actief VWF in het bloed van deze patiënten en in de invloed van het stadium van nierinsufficiëntie en het type dialyse. In deze studie, beschreven in hoofdstuk 7, vonden we inderdaad verhoogde actief VWF waarden in alle patiëntengroepen (chronische nierinsufficiëntie, eindstadium nierfalen met hemodialyse of peritoneaal dialyse) ten opzichte van gezonde controles. ${ }^{5}$ Ook totaal VWF was verhoogd maar de verschillen tussen de groepen 
waren minder groot. Er was een zichtbaar toenemende trend van actief VWF, maar niet van VWF antigeen (totaal VWF), met de ernst van de nierinsufficiëntie. Het achterliggende mechanisme is waarschijnlijk chronische blootstelling van het endotheel aan inflammatoire cytokines en oxidatieve stress, waardoor steeds meer endotheel schade ontstaat en UL-VWF vrijkomt in de circulatie. Bovendien vond een eerdere studie in patiënten met chronische nierinsufficiëntie verminderde ADAMTS13 activiteit (het enzym dat UL-VWF in kleinere fragmenten knipt) in vergelijking met controles. ${ }^{6}$ Daardoor zal een deel van het UL-VWF niet worden geknipt en in zijn actieve conformatie blijven. Eerder werd aangetoond dat hoge concentraties VWF een voorspeller zijn voor microalbuminurie, hart-vaat ziekten en mortaliteit in deze patiëntengroep. Op basis van onze resultaten zouden actief VWF levels wellicht nog sterker geassocieerd kunnen zijn met deze klinische uitkomsten. Grotere studies met klinische follow-up moeten aantonen of dit daadwerkelijk het geval is.

Terwijl de meeste studies naar actief VWF focussen op ziekten die geassocieerd zijn met trombotische complicaties (door verhoogd actief VWF), kan spontane interactie tussen actief VWF en plaatjes de hemostatische balans ook doen doorslaan naar de bloedingskant, zoals in von Willebrand ziekte type $2 \mathrm{~B}$. Het is echter niet bekend of de fractie van actief VWF in de circulatie ook een rol speelt in patiënten met een milde bloedingsneiging zonder trombocytopenie. De diagnose van deze patiënten is erg lastig en in veel gevallen wordt de onderliggende oorzaak nooit gevonden. In hoofdstuk 8 hebben we daarom actief VWF bepaald in een populatie van 109 patiënten met een milde bloedingsdiathese. Actief VWF correleerde in deze populatie significant met de andere VWF parameters, net als in gezonde individuen (zie hoofdstuk 3). Actief VWF en VWF antigeen levels, alsook VWF ristocetine cofactor activiteit, waren relatief lager dan de vastgestelde referentie intervallen, maar dit was niet significant. Actief VWF was ook niet significant verschillend tussen individuen met en zonder verhoogde klinische bloedingsscore (ISTH-BAT) en tussen individuen met een verdenking op de ziekte van von Willebrand versus andere oorzaken van de bloedingsneiging. Met deze studie tonen we aan dat kwantificeren van actief VWF geen diagnostische meerwaarde heeft naast de gevestigde VWF antigeen en VWF ristocetine cofactor activiteit assays in deze populatie.

Eerdere studies hebben aangetoond dat naast ADAMTS13 ook plasmine VWF kan knippen. ${ }^{7.8}$ Om meer inzicht te verwerven in de effecten van plasmine op (actief) VWF, plaatjes en hun interactie hebben we een grondige literatuurstudie gedaan, beschreven in hoofdstuk 9 van dit proefschrift. Interessant genoeg wordt er meer plasmine gevormd in patiënten tijdens een acute episode van TTP, hetgeen suggereert dat plasmine als "back-up" mechanisme voor ADAMTS13 zou kunnen werken om grote UL-VWF 
multimeren te klaren. ${ }^{7}$ Het toedienen van trombolytica (recombinant tPA) zou mogelijk kunnen werken om tijdelijk hogere concentraties plasmine te genereren. Naast het knippen van VWF en zijn primaire substraat fibrine heeft plasmine nog veel andere effecten, waaronder proteolyse van een groot aantal andere stolfactoren. ${ }^{9}$ Daarnaast beïnvloedt plasmine de bloedplaatjesactiviteit, o.a. door het knippen van verschillende receptoren betrokken bij plaatjes activatie (PAR-4), adhesie en aggregatie (GP1b, GPIIbllla). Hoewel het onwaarschijnlijk is dat plasmine-gemedieerde plaatjesactivatie een rol speelt bij fysiologische hemostase, zouden deze effecten wel van belang kunnen zijn na het toedienen van trombolytica, aangezien de concentratie van plasmine dan supra-fysiologisch hoog is. Mechanistische studies zijn nodig om een beter begrip te krijgen van de effecten van plasmine op bloedplaatjes activatie en binding aan (actief) VWF.

Tot slot worden in hoofdstuk 10 de belangrijkste conclusies samengevat en geplaatst in de context van de literatuur. Ook worden mogelijke implicaties van onze bevindingen en richtingen voor toekomstig onderzoek besproken. Neutralisatie van de interactie tussen bloedplaatjes en VWF is een potentiële strategie ter preventie/behandeling van hemostatische complicaties veroorzaakt door verhoogde hoeveelheden circulerend actief VWF. De eerste in vitro experimenten met S-VWFA1 duiden op mogelijke voordelige eigenschappen van deze $\mathrm{VHH}$, namelijk het blokkeren van bloedplaatjes binding aan VWF in oplossing maar niet aan collageen-gebonden VWF. In vivo experimenten in diermodellen zijn nodig om te bepalen of S-VWFA1 daadwerkelijk excessieve plaatjesVWF interactie (zoals in TTP en de ziekte van von Willebrand type 2B) kan oplossen. Daarnaast bevestigen de bevindingen in COPD en CKD patiënten dat het kwantificeren van actief VWF in plasma informatie kan geven over de onderliggende oorzaken van een verhoogd trombotisch risico. Echter, er zijn grote, klinische uitkomst studies nodig om vast te stellen wat de meerwaarde is van actief VWF naast bestaande VWF assays (o.a. VWF antigeen, ristocetine cofactor activiteit, VWF-GPIbM binding) als biomarker voor hart- en vaatziekten, in de diagnostiek en/of de monitoring van therapie. 


\section{REFERENTIES}

1. van der Vorm LN, Li L, Huskens D, Chayoua W, Kelchtermans H, de Groot PG, et al. Analytical characterization and reference interval of an enzyme-linked immunosorbent assay for active von Willebrand factor. PLoS One. 2019;14(2):e0211961.

2. van der Vorm LN, Huskens D, Kicken CH, Remijn JA, Roest M, de Laat B, et al. Effects of Repeated Bouts of Exercise on the Hemostatic System. Semin Thromb Hemost. 2018;44(8):710-22.

3. Kicken $\mathrm{CH}$, van der Vorm LN, Zwaveling S, Schoenmaker E, Remijn JA, Huskens D, et al. A Hypoxic Environment Attenuates Exercise-Induced Procoagulant Changes Due to Decreased Platelet Activation. TH Open. 2019;3(3):e216-e26.

4. Huang MJ, Wei RB, Wang Y, Su TY, Di P, Li OP, et al. Blood coagulation system in patients with chronic kidney disease: a prospective observational study. BMJ Open. 2017;7(5):e014294.

5. van der Vorm L, Visser R, Huskens D, Veninga A, Adams D, Remijn J, et al. Circulating active von Willebrand factor levels are increased in chronic kidney disease and end-stage renal disease. CKJ. 2019:1-3.

6. Shen L, Lu G, Dong N, Jiang L, Ma Z, Ruan C. Von Willebrand factor, ADAMTS13 activity, TNF-alpha and their relationships in patients with chronic kidney disease. Exp Ther Med. 2012;3(3):530-4.

7. Tersteeg C, de Maat S, De Meyer SF, Smeets MW, Barendrecht AD, Roest M, et at. Plasmin cleavage of von Willebrand factor as an emergency bypass for ADAMTS13 deficiency in thrombotic microangiopathy. Circulation. 2014;129(12):1320-31.

8. Brophy TM, Ward SE, McGimsey TR, Schneppenheim S, Drakeford C, O'Sullivan JM, et al. Plasmin Cleaves Von Willebrand Factor at K1491-R1492 in the A1-A2 Linker Region in a Shearand Glycan-Dependent Manner In Vitro. Arterioscler Thromb Vasc Biol. 2017;37(5):845-55.

9. van der Vorm LN, Remijn JA, De Laat B, Huskens D. Effects of Plasmin on von Willebrand Factor and Platelets: A Narrative Review. TH Open. 2018;2(2):e218-e28. 
Appendix I 


\section{Valorisation}





\section{VALORISATION}

The major goal of this thesis was to explore the role of active VWF in physiological haemostasis and pathological conditions associated with an increased risk of thrombosis (strenuous exercise, COPD, CKD) or a mild bleeding phenotype. This chapter first provides a short overview of the societal and economic burden of the overarching "problem" of cardiovascular disease. This is followed by a discussion of the potential opportunities for valorisation of the knowledge obtained through our studies, with valorisation referring to "the process of creating value from knowledge, making knowledge suitable and/or available for economic and social use and for translation into competitive products, services, processes and new activities". ${ }^{1}$

\section{Cardiovascular disease: a deadly and costly burden}

Cardiovascular diseases (CVD) represent the leading cause of death worldwide, with more than 4 million deaths each year. ${ }^{2}$ Direct and indirect costs of CVD account for approximately $12 \%$ of the total European health care expenditures. ${ }^{3}$ Thrombosis is the most common underlying pathology of the three major cardiovascular disorders: ischemic heart disease (IHD), stroke, and venous thromboembolism (VTE), which includes both deep vein thrombosis (DVT) and pulmonary embolism (PE). Together, the two leading forms of arterial thrombosis, IHD (7 million deaths) and stroke (5.9 million deaths) collectively cause one in four deaths worldwide ${ }^{4}$, while epidemiological studies estimate the number of VTE-related deaths to be around 500.000 per year in the European Union alone. ${ }^{5}$ Estimates of the total economic impact of VTE, taking into account the lost economic output due to premature mortality, are as high as $\$ 69$ billion per year, and will continue to rise due to increasing longevity. ${ }^{6}$ The numbers presented here reflect the enormous disease- and economic burden of thrombosis, and hence project the potential benefits of prevention, in terms of reducing mortality/loss of productivity and healthcare costs. The subsequent paragraphs of this chapter will focus on how our findings may be relevant for society, therapy- and prevention strategies and diagnostic laboratory practice in the field of thrombosis and haemostasis.

\section{Neutralization of the (active) VWF-platelet interaction}

Spontaneous binding of platelets to active VWF occurs in a variety of diseases and can lead to thrombotic and/or bleeding complications that have a large impact on the quality of life, morbidity and mortality. In a number of common, multifactorial conditions associated with a prothrombotic phenotype, (e.g. COPD (Chapter 6) and CKD (Chapter 7)), increased active VWF levels are certainly not the only cause of the increased thrombotic risk. However, in some (generally rarer) conditions, active VWF is causally implicated in the haemostatic complications, such as in TTP (prevalence 1-13 per million ${ }^{7}$ ), VWD 
type $2 \mathrm{~B}\left(<<1\right.$ per $\left.10.000^{8}\right)$ and HELLP $\left(0.17-0.85 \%\right.$ of all live births $\left.{ }^{9}\right)$. Despite their low prevalence, the economic burden of these conditions is substantial. Exacerbations of acute TTP are treated with plasma exchange in conjunction with immunosuppression to remove UL-VWF and inhibitory autoantibodies, replenish ADAMTS13 and suppress new antibody production. ${ }^{10}$ Average healthcare costs for a hospitalization for incident acute TTP in the US were previously estimated to be $\$ 56.347$ (SD \$80.230), and are even higher in patients requiring prolonged plasma exchange. ${ }^{11}$ For VWD type $2 \mathrm{~B}$, replacement therapy with purified VWF/FVIII concentrates is required to control recurrent bleeding and associated complications. The direct and indirect costs of such VWF/FVIII concentrate therapy is around $€ 250.000$ per year. ${ }^{12}$ Also in more prevalent diseases, such as malaria ( 219 million cases worldwide ${ }^{13}$ ) and dengue ( 96 million symptomatic, worldwide ${ }^{14}$ ), evidence demonstrates a link between active (UL-)VWF and haemostatic complications. These diseases mainly occur in developing countries with limited healthcare resources.

Neutralization of the interaction between VWF and platelets appears to be an attractive strategy to prevent microthrombi formation (as in TTP) and excessive platelet clearance (with resulting thrombocytopenia and bleeding), thereby reducing the disease burden and societal and economic impact. This strategy was previously explored by others (Chapter 10, Table 1), of which the bivalent VHH caplacizumab is the most successful. ${ }^{15,16}$ As demonstrated in Chapter 2, our VHH S-VWFA1 could have beneficial properties over caplacizumab as it specifically inhibits only active VWF. However, it is debatable whether one should pursue the development of this $\mathrm{VHH}$ and the possible ensuing clinical studies in light of the financial burden of drug development programmes (estimated to be around 200-400 million US dollars ${ }^{17}$ ), given that caplacizumab is already available and approved, and TTP is a rare ("ultra-orphan") disease. In addition, the increased bleeding risk associated with caplacizumab appears mild and manageable and only required VWF/FVIII concentrate replacement therapy in a small percentage of patients in the phase III HERCULES trial. ${ }^{10}$ Of note, the costs for treating a typical acute TTP episode with caplacizumab (Cabliviß) is $\$ 270,000$. One argument in favour of our VHH is that it would be theoretically more suitable for prophylactic long-term use than caplacizumab. Caplacizumab induces increased clearance of VWF and FVIII, which would ultimately lead to a more severe bleeding risk, ${ }^{15}$ while targeting only active VWF would allow for prevention (instead of rescuing) of acute TTP episodes. This would also reduce the associated costs of hospitalization and other treatment modalities.

Hypothetically, caplacizumab may also benefit patients with other conditions/complications related to excessive platelet-VWF interactions and consequent thrombocytopenia. In conditions with a bleeding phenotype, such as VWD type $2 \mathrm{~B}$, the increased 
bleeding risk associated with caplacizumab treatment needs extra consideration. In these conditions, the S-VWFA1 VHH, which is expected to pose no/less risk of bleeding due to its specificity for active VWF, may be beneficial in terms of safety.

Altogether, in terms of valorisation, there are still many open questions around the possible "market size" of the VHH and its value proposition (since an alternative, caplacizumab, is available). Given the very early stage of its development, significant resources would be needed to reach a viable and valuable point for patent application. It is uncertain whether the potential benefits of the S-VWFA1 VHH outweigh the costs associated with drug development.

\section{Raising awareness on the prothrombotic effects of physical stress}

Compelling evidence demonstrates that physical activity reduces premature mortality and prevents a variety of chronic medical conditions, including CVD. ${ }^{18,19}$ However, there is also evidence of sudden cardiac death and thromboembolic events in athletes, and clearly, there are risks associated with vigorous and/or prolonged physical activity. ${ }^{20}$ Although our findings confirm previous studies on the prothrombotic response to exercise $^{21}$, in particular of the endothelium, these experimental findings in small groups of healthy individuals may not directly have a socio-economic impact. Nevertheless, future larger studies may be able to resolve some of the important gaps in knowledge (as discussed in Chapter 10) and contribute to the public awareness of the thrombotic risks associated with strenuous exercise in untrained individuals.

Research into the effects of exercise is (clinically) relevant for several situations and groups of individuals. Although our studies focused on the effects of cycling on coagulation, other forms of exercise, such as (long-distance) running, may cause similar effects. Running is one of the world's most popular sports ${ }^{22}$ and marathon events continue to grow annually ${ }^{22}$ (called "marathon fever"), especially among middle-aged, non-elite runners. ${ }^{23}$ An increased frequency of sudden cardiac death has been observed in middle-aged men during marathons since the year $2000.24,25$ Atherosclerotic heart disease was the main cause of marathon-related sudden cardiac death in participants over the age of $40 .{ }^{26}$ These studies clearly demonstrate an increase in marathon-related cardiac arrests in middle-aged men, while there is a declining rate of sudden cardiac deaths in the general population. ${ }^{27}$ Siegel et al. previously studied inflammatory- and coagulation markers in pre- and post-race blood samples of middle-aged male participants of the Boston marathon. ${ }^{28}$ Post-race samples showed neutrophilia and elevated interleukin- 6 (IL-6) and C reactive protein (CRP), in combination with elevated fibrinogen, VWF, Ddimer and platelet activation, together indicating a pro-inflammatory and procoagulant state. ${ }^{28}$ Protection of susceptible runners from atherosclerotic complications during 
marathons may be feasible by low-dose aspirin before the race. ${ }^{29}$ Although our findings indicate that endothelial activation appears more important for the procoagulant state during/following exercise than platelet activation (which is targeted by aspirin), aspirin also has anti-inflammatory effects, which may reduce endothelial activation as well. Thus, pre-race low-dose aspirin may provide antithrombotic benefit for "at-risk" runners during the race and for the 24 hours of high post-race cardiac risk.

\section{Quantification of active VWF in thrombo-inflammatory conditions}

The socio-economic burden of COPD and CKD

In Chapters 6 and 7, we studied active VWF (and other haemostatic parameters) in two thrombo-inflammatory conditions, namely COPD and CKD. Both are chronic, progressive conditions with a high prevalence: the Global Burden of Disease Study reported a prevalence of 251 million cases of COPD and 752.7 million people with impaired kidney function globally in 2016. ${ }^{30,31}$ Not surprisingly, both are also associated with a tremendous economic burden. For COPD patients, the periodic deteriorations called exacerbations pose the highest financial burden due to supplementary treatment and increased hospital admissions. ${ }^{32}$ With regard to CKD, high-income countries typically spend more than $2-3 \%$ of their annual healthcare budget on the treatment of endstage kidney disease. ${ }^{33}$

\section{Active VWF in COPD and CKD}

Importantly, both conditions are independently associated with an increased risk of thrombotic cardiovascular events, which contribute significantly to the overall disease morbidity and mortality. ${ }^{34,35}$ As endothelial activation in response to the inflammatory state in these conditions is known to contribute to the thrombotic risk, we assessed whether circulating active VWF levels were increased in patients with COPD (Chapter 6) and CKD (Chapter 7). This was indeed confirmed ${ }^{36}$, and is likely a consequence of the increased release of VWF by the inflamed endothelium in combination with decreased ADAMTS13 activity, due to inhibition by inflammatory mediators and saturation with the excessive VWF antigen. ${ }^{37}$ However, it is important to emphasize that in these multifactorial conditions active VWF is only one of the various haemostatic factors that are altered in response to the chronic inflammatory state, as discussed in Chapter 10.

\section{Diagnostic value of active VWF}

One important question with regard to valorisation is: is there added (diagnostic) value of measuring active VWF levels, and if so, in which conditions? In valorisation terms, this question pertains to the value proposition: which problem does active VWF quantification solve? Based on the discussion in Chapter 10, the conclusion would be that large, clinical outcome studies need to be performed first to answer the question on 
the clinical usefulness of active VWF measurements. Current evidence only supports a possible role of active VWF measurements in the monitoring of TTP patients. ${ }^{15,16,38,39}$ In most conditions, including mild bleeding disorders (Chapter 8 ) active VWF correlates well with established laboratory tests (VWF antigen, ristocetin cofactor activity) ${ }^{40}$, but the additional diagnostic value is not clear at the moment.

\section{Commercially available active VWF kit for research?}

Despite the current absence of large clinical outcome studies, measuring active VWF levels has proven very valuable in unraveling the pathophysiological mechanisms of haemostatic complications in a variety of conditions. ${ }^{41-45}$ Currently, the value of active VWF quantification thus seems to lie in the research field. Thus, possible "customers" would be research groups studying conditions associated with increased thrombotic risk or bleeding tendency due to thrombocytopenia. It is therefore important to make our assay (Chapter 3) widely available to other researchers, for instance in the form of a "kit" with pre-coated ELISA plates and all required buffers and antibodies. Previous experiments (unpublished data) have shown that coating of the $\mathrm{VHH}$ in a buffer containing trehalose to (vacuum sealed) ELISA plates provided good stability for at least 1 month when stored at $4^{\circ} \mathrm{C}$. Further validation studies of these active VWF kits are required, but may ultimately lead to a commercially available kit.

\section{Active VWF in clinical laboratories?}

If large clinical outcome studies would prove additional diagnostic value for active VWF quantification, implementation in the clinical chemistry laboratory requires significant modifications to the current manual ELISA assay. In this form, the assay is labour-intensive and time-consuming. Thus, a high-throughput assay that can be run on existing automated laboratory analysers should be developed to make the test "hospital laboratory-friendly". Regarding market potential, possible partners for codevelopment would be large companies specialized in the development of laboratory assays and analysers in the field of haemostasis. Besides the automation of the assay, an important aspect to consider is the comparability of results between laboratories. ${ }^{46}$ The active VWF assay uses normal pooled plasma as a reference to which sample results are normalized, which may result in between-laboratory bias, as discussed in Chapters 3 and 10. Thus, it is important that the potential manufacturer of the assay develops a (lyophilized) reference plasma for normalization of assay results. ${ }^{47}$ This allows for comparison of results between laboratories and hence patient populations. 


\section{CONCLUSION}

Most of the research presented in this thesis should be regarded as explorative work, with the aim to generate hypotheses for future, more large-scale studies. Although there is a long road ahead to fully explore the therapeutic and diagnostic opportunities of the S-VWFA1 and active VWF assay, our findings are the first steps in that direction, and may hence guide research that will have a socio-economic impact in the future. 


\section{REFERENCES}

1. Vereniging van Nederlandse Universiteiten (VSNU). Een Raamwerk Valorisatie-indicatoren. Den Haag: VSNU; 2013.

2. Wendelboe AM, Raskob GE. Global Burden of Thrombosis: Epidemiologic Aspects. Circ Res. 2016;118(9):1340-7.

3. Leal J, Luengo-Fernandez R, Gray A, Petersen S, Rayner M. Economic burden of cardiovascular diseases in the enlarged European Union. Eur Heart J. 2006;27(13):1610-9.

4. Lozano R, Naghavi M, Foreman K, Lim S, Shibuya K, Aboyans V, et al. Global and regional mortality from 235 causes of death for 20 age groups in 1990 and 2010: a systematic analysis for the Global Burden of Disease Study 2010. Lancet. 2012;380(9859):2095-128.

5. Cohen AT, Agnelli G, Anderson FA, Arcelus JI, Bergqvist D, Brecht JG, et al. Venous thromboembolism (VTE) in Europe. The number of VTE events and associated morbidity and mortality. Thromb Haemost. 2007;98(4):756-64.

6. Mahan CE, Borrego ME, Woersching AL, Federici R, Downey R, Tiongson J, et al. Venous thromboembolism: annualised United States models for total, hospital-acquired and preventable costs utilising long-term attack rates. Thromb Haemost. 2012;108(2):291-302.

7. Reese JA, Muthurajah DS, Kremer Hovinga JA, Vesely SK, Terrell DR, George JN. Children and adults with thrombotic thrombocytopenic purpura associated with severe, acquired Adamts13 deficiency: comparison of incidence, demographic and clinical features. Pediatr Blood Cancer. 2013;60(10):1676-82.

8. Sadler JE. A revised classification of von Willebrand disease. For the Subcommittee on von Willebrand Factor of the Scientific and Standardization Committee of the International Society on Thrombosis and Haemostasis. Thromb Haemost. 1994;71(4):520-5.

9. Rath W, Faridi A, Dudenhausen JW. HELLP syndrome. J Perinat Med. 2000;28(4):249-60.

10. Scully M, Cataland SR, Peyvandi F, Coppo P, Knobl P, Kremer Hovinga JA, et al. Caplacizumab Treatment for Acquired Thrombotic Thrombocytopenic Purpura. N Engl J Med. 2019;380(4):335-46.

11. Wahl PM, Bohn RL, Terrell DR, George JN, Ewenstein B. Health care utilization of patients diagnosed with idiopathic thrombotic thrombocytopenic purpura in a commercially insured population in the United States. Transfusion. 2012;52(7 Pt 2):1614-21.

12. Schinco P, Cultrera D, Valeri F, Borchiellini A, Mantuano M, Gorla F, et al. Cost-consequence analysis of long-term prophylaxis in the treatment of von Willebrand disease in the Italian context. Clinicoecon Outcomes Res. 2015;7:17-25.

13. World Health Organization. World Malaria Report Geneva, Switzerland; 2018.

14. Bhatt S, Gething PW, Brady OJ, Messina JP, Farlow AW, Moyes CL, et al. The global distribution and burden of dengue. Nature. 2013;496(7446):504-7.

15. Peyvandi F, Scully M, Kremer Hovinga JA, Cataland S, Knobl P, Wu H, et al. Caplacizumab for Acquired Thrombotic Thrombocytopenic Purpura. N Engl J Med. 2016;374(6):511-22.

16. Peyvandi F, Scully M, Kremer Hovinga JA, Knobl P, Cataland S, De Beuf K, et al. Caplacizumab reduces the frequency of major thromboembolic events, exacerbations and death in patients with acquired thrombotic thrombocytopenic purpura. J Thromb Haemost. 2017;15(7):1448-52.

17. Jayasundara K, Hollis A, Krahn M, Mamdani M, Hoch JS, Grootendorst P. Estimating the clinical cost of drug development for orphan versus non-orphan drugs. Orphanet J Rare Dis. 2019;14(1):12. 
18. Shiroma EJ, Lee IM. Physical activity and cardiovascular health: lessons learned from epidemiological studies across age, gender, and race/ethnicity. Circulation. 2010;122(7):743-52.

19. Swift DL, Lavie CJ, Johannsen NM, Arena R, Earnest CP, O'Keefe JH, et al. Physical activity, cardiorespiratory fitness, and exercise training in primary and secondary coronary prevention. Circ J. 2013;77(2):281-92.

20. Kicken $\mathrm{CH}$, Miszta A, Kelchtermans H, De Laat B. Hemostasis during Extreme Exertion. Semin Thromb Hemost. 2018;44(7):640-50.

21. Huskens D, Roest M, Remijn JA, Konings J, Kremers RM, Bloemen S, et al. Strenuous exercise induces a hyperreactive rebalanced haemostatic state that is more pronounced in men. Thromb Haemost. 2016;115(6):1109-19.

22. Nikolaidis PT, Rosemann T, Knechtle B. Sex Differences in the Age of Peak Marathon Race Time. Chin J Physiol. 2018;61(2):85-91.

23. Summers J, Sargent G, Levey A, Murray K. Middle-aged, non-elite marathon runners: a profile. . Percept Mot Skills. 1982;54:963-9.

24. Mathews SC, Narotsky DL, Bernholt DL, Vogt M, Hsieh YH, Pronovost PJ, et al. Mortality among marathon runners in the United States, 2000-2009. Am J Sports Med. 2012;40(7):1495500.

25. Kim JH, Malhotra R, Chiampas G, d'Hemecourt P, Troyanos C, Cianca J, et al. Cardiac arrest during long-distance running races. N Engl J Med. 2012;366(2):130-40.

26. Harmon KG, Drezner JA, Wilson MG, Sharma S. Incidence of sudden cardiac death in athletes: a state-of-the-art review. Br J Sports Med. 2014;48(15):1185-92.

27. Benjamin EJ, Muntner P, Alonso A, Bittencourt MS, Callaway CW, Carson AP, et al. Heart Disease and Stroke Statistics-2019 Update: A Report From the American Heart Association. Circulation. 2019;139(10):e56-e528.

28. Siegel AJ, Stec JJ, Lipinska I, Van Cott EM, Lewandrowski KB, Ridker PM, et al. Effect of marathon running on inflammatory and hemostatic markers. Am J Cardiol. 2001;88(8):918-20, A9.

29. Siegel AJ. Prerace aspirin to protect susceptible runners from cardiac arrest during marathons: is opportunity knocking? Open Heart. 2015;2(1):e000102.

30. WHO Fact Sheet: Chronic Obstructive Pulmonary Disease (COPD). World Health Organization; 2017.

31. Bikbov B, Perico N, Remuzzi G, on behalf of the GBDGDEG. Disparities in Chronic Kidney Disease Prevalence among Males and Females in 195 Countries: Analysis of the Global Burden of Disease 2016 Study. Nephron. 2018;139(4):313-8.

32. Sullivan SD, Ramsey SD, Lee TA. The economic burden of COPD. Chest. 2000;117(2 Suppl):5S-9S.

33. Couser WG, Remuzzi G, Mendis S, Tonelli M. The contribution of chronic kidney disease to the global burden of major noncommunicable diseases. Kidney Int. 2011;80(12):1258-70.

34. Anthonisen NR, Connett JE, Enright PL, Manfreda J, Lung Health Study Research G. Hospitalizations and mortality in the Lung Health Study. Am J Respir Crit Care Med. 2002;166(3):3339.

35. Wattanakit K, Cushman M, Stehman-Breen C, Heckbert SR, Folsom AR. Chronic kidney disease increases risk for venous thromboembolism. J Am Soc Nephrol. 2008;19(1):135-40.

36. van der Vorm L, Visser R, Huskens D, Veninga A, Adams D, Remijn J, et al. Circulating active von Willebrand factor levels are increased in chronic kidney disease and end-stage renal disease. CKJ. 2019:1-3. 
37. Bernardo A, Ball C, Nolasco L, Moake JF, Dong JF. Effects of inflammatory cytokines on the release and cleavage of the endothelial cell-derived ultralarge von Willebrand factor multimers under flow. Blood. 2004;104(1):100-6.

38. Sargentini-Maier ML, De Decker P, Tersteeg C, Canvin J, Callewaert F, De Winter H. Clinical pharmacology of caplacizumab for the treatment of patients with acquired thrombotic thrombocytopenic purpura. Expert Rev Clin Pharmacol. 2019;12(6):537-45.

39. Groot E, de Groot PG, Fijnheer R, Lenting PJ. The presence of active von Willebrand factor under various pathological conditions. Curr Opin Hematol. 2007;14(3):284-9.

40. van der Vorm LN, Li L, Huskens D, Chayoua W, Kelchtermans H, de Groot PG, et al. Analytical characterization and reference interval of an enzyme-linked immunosorbent assay for active von Willebrand factor. PLoS One. 2019;14(2):e0211961.

41. de Mast $Q$, Groot E, Asih PB, Syafruddin D, Oosting M, Sebastian S, et al. ADAMTS13 deficiency with elevated levels of ultra-large and active von Willebrand factor in P. falciparum and P. vivax malaria. Am J Trop Med Hyg. 2009;80(3):492-8.

42. de Mast $\mathrm{Q}$, Groot E, Lenting PJ, de Groot PG, McCall M, Sauerwein RW, et al. Thrombocytopenia and release of activated von Willebrand Factor during early Plasmodium falciparum malaria. J Infect Dis. 2007;196(4):622-8.

43. Djamiatun K, van der Ven AJ, de Groot PG, Faradz SM, Hapsari D, Dolmans WM, et al. Severe dengue is associated with consumption of von Willebrand factor and its cleaving enzyme ADAMTS-13. PLoS Negl Trop Dis. 2012;6(5):e1628.

44. Hulstein JJ, Lenting PJ, de Laat B, Derksen RH, Fijnheer R, de Groot PG. beta2-Glycoprotein I inhibits von Willebrand factor dependent platelet adhesion and aggregation. Blood. 2007;110(5):1483-91.

45. Hulstein JJ, van Runnard Heimel PJ, Franx A, Lenting PJ, Bruinse HW, Silence K, et al. Acute activation of the endothelium results in increased levels of active von Willebrand factor in hemolysis, elevated liver enzymes and low platelets (HELLP) syndrome. J Thromb Haemost. 2006;4(12):2569-75.

46. Tate J, Panteghini M. Standardisation--the theory and the practice. Clin Biochem Rev. 2007;28(4):127-30.

47. ISO. In vitro diagnostic medical devices - measurement of quantities in biological samples - metrological traceability of values assigned to calibrators and control materials. 1st ed. Geneva: ISO; 2003. ISO 17511:2003. 


$$
\text { Appendix II }
$$




\section{Curriculum Vitae}





\section{CURRICULUM VITAE}

Lisa Nynke van der Vorm was born on 25 October 1993 in Apeldoorn, the Netherlands. Between 2005 and 2011, she attended pre-university secondary education (VWO) at the Koninklijke Scholengemeenschap Apeldoorn and graduated cum laude. Subsequently, Lisa studied Biomedical Sciences at the Radboud University in Nijmegen, the Netherlands. During her studies, she spent several months abroad. For her bachelor internship in 2014, she studied 4 months at the Department of Medicinal Chemistry of King's College (London, UK), where her project focused on the detection and quantification of non-transferrin-bound iron in plasma of patients suffering from iron overload. In 2016, she studied for 7 months at Harvard Medical School/Brigham and Women's Hospital (Boston, USA), where she studied the functional consequences of a genetic mutation causing a rare disorder of haem metabolism. After her cum laude graduation, she started her PhD programme at Synapse Research Institute, affiliated to Maastricht University. During the first year of her PhD she lived and worked in Maastricht, focusing on the validation of an ELISA assay for the quantification of active von Willebrand Factor (VWF) in plasma. For the remainder of her PhD programme she moved to Apeldoorn and started working in the Gelre Ziekenhuizen, where she used the developed assay to study active VWF in several patient populations. Lisa is currently living in Apeldoorn with her partner Emir. After obtaining her PhD degree she will start her resident training in Clinical Chemistry at the Amsterdam University Medical Centre, location AMC. 
Appendix III 


\section{List of publications}





\section{LIST OF PUBLICATIONS}

\section{Publications in this thesis}

van der Vorm LN, Huskens D, Kicken CH, et al. Effects of Repeated Bouts of Exercise on the Hemostatic System. Semin Thromb Hemost. 2018;44(8):710-722.

van der Vorm LN, Remijn JA, de Laat B, Huskens D. Effects of Plasmin on von Willebrand Factor and Platelets: A Narrative Review. TH Open. 2018; 02(02): e218-e228

van der Vorm LN, Li L, Huskens D, Chayoua W, Kelchtermans H, de Groot PG, Roest $M$, Remijn JA, de Laat B. Analytical characterization and reference interval of an enzyme-linked immunosorbent assay for active von Willebrand factor. PLoS One. 2019;14(2):e0211961.

Kicken CH, van der Vorm LN, Zwaveling S, Schoenmaker E, Remijn JA, Huskens D, de Laat B. A Hypoxic Environment Attenuates Exercise-Induced Procoagulant Changes Due to Decreased Platelet Activation. TH Open. 2019; 3(3): e216-e226.

van der Vorm LN, Visser R, Huskens D, Veninga A, Adams DL, Remijn JA, Hemker HC, Rensma PL, van Horssen $\mathrm{R}$, de Laat $\mathrm{B}$. Circulating active von Willebrand factor levels are increased in chronic kidney disease and end-stage renal disease. Clinical Kidney Journal. 2019; sfz076.

van der Vorm LN, Huskens D, Florin L, De Kesel P, Roest M, de Laat B, Devreese KMJ. Active von Willebrand Factor in patients with a bleeding diathesis. Thrombosis Update. 2020;1

van der Vorm LN, Li L, Huskens D, Hulstein JJ], Roest M, de Groot PG, ten Cate H, de Laat B, Remijn JA, Simons SO. Acute exacerbations of COPD induce a prothrombotic state through platelet-monocyte complexes, endothelial activation and increased thrombin generation. Submitted.

van der Vorm LN, Huskens D, Konings J, Roest M, de Groot PG, de Laat B, Remijn JA. $A$ variable heavy chain antibody against active von Willebrand factor only inhibits platelet-VWF interaction in solution. Manuscript in preparation. 


\section{Other publications}

Huskens D, Sang Y, Konings J, van der Vorm L, de Laat B, Kelchtermans H, Roest M. Standardization and reference ranges for whole blood platelet function measurements using a flow cytometric platelet activation test. PLOS One. 2018;13(2):e0192079.

Spronk HMH, Padro T, Siland JE, Prochaska JH, Winters J, van der Wal AC, Posthuma JJ, Lowe G, d'Alessandro E, Wenzel P, Coenen DM, Reitsma PH, Ruf W, van Gorp RH, Koenen RR, Vajen T, Alshaikh NA, Wolberg AS, Macrae FL, Asquith N, Heemskerk J, Heinzmann A, Moorlag M, Mackman N, van der Meijden P, Meijers JCM, Heestermans M, Renné T, Dólleman S, Chayouâ W, Ariëns RAS, Baaten CC, Nagy M, Kuliopulos A, Posma J], Harrison P, Vries MJ, Crijns HJGM, Dudink EAMP, Buller HR, Henskens YMC, Själander A, Zwaveling S, Erküner O, Eikelboom JW, Gulpen A, Peeters FECM, Douxfils J, Olie RH, Baglin T, Leader A, Schotten U, Scaf B, van Beusekom HMM, Mosnier LO, van der Vorm L, Declerck P, Visser M, Dippel DWJ, Strijbis VJ, Pertiwi K, Ten Cate-Hoek AJ, Ten Cate H. Atherothrombosis and Thromboembolism: Position Paper from the Second Maastricht Consensus Conference on Thrombosis. Thromb Haemost. 2018;118(2):229-250.

van der Vorm LN, Brouwers J, Mondria C, de Laat B, de Groot PG, Remijn JA. Salivary tissue factor induces thrombin generation in a diurnal rhythm. Res Pract Thromb Haemost. 2018;2(4):757-761.

Brouwers JEIG, van der Vorm LN, Buis S, Haumann R, Karanzai A, Konings J, de Groot PG, de Laat B, Remijn JA. Implant stability in patients treated with platelet-rich fibrin and bovine bone substitute for alveolar ridge preservation is associated with peripheral blood cells and coagulation factors. Clin Exp Dent Res. 2019; 1-8.

Aleva FE, Tunjungputri RN, van der Vorm LN, Li Y, Heijdra YF, Oosting M, Smeekens SP, Jaeger M, Joosten LAB, de Groot PhG, Netea MG, van der Ven AJAM and de Mast O.. Platelet integrin $\alpha$ llb $\beta 3$ activation is associated with 25 -hydroxyvitamin $D$ concentrations in healthy adults. Accepted for publication, Thromb Haemost. 2020

de Laat-Kremers R, Di Castelnuovo A, van der Vorm L, Costanzo S, Ninivaggi M, Cerletti C, Huskens D De Curtis A, Gialluisi A, Bai C, de Gaetano G, Yin D, Donati MB, de Laat B, lacoviello L. Increased BMI and Blood Lipids are Associated with Procoagulant Thrombin Generation Profile in the Moli-sani Cohort. Submitted. 2020.

\section{Oral and poster presentations}

van der Vorm LN, Huskens D, Remijn JA, de Laat B. Inhibition of thrombin generation distinguishes low and high responders to unfractionated heparin. International Society 
on Thrombosis and Hemostasis (ISTH) conference, Berlin, Germany, 2017 (poster presentation).

van der Vorm LN, Miszta A, Roest M, Remijn JA, Huskens D, de Laat B. Characterization of a Nanobody That Binds VWF and Prevents Von Willebrand Factor Cleavage by Plasmin Poster presentation at the American Society of Hematology (ASH) Annual Meeting, Atlanta, USA, 2017 (poster presentation).

van der Vorm LN, Brouwers J, Mondria C, de Laat B, de Groot PG, Remijn JA. Saliva-derived tissue factor induces thrombin generation in a diurnal rhythm. Scientific and Standardization Committee (SSC)/ISTH conference, Dublin, Ireland, 2018 (poster presentation).

van der Vorm LN, Visser R, Huskens D, Veninga A, Remijn JA, Hemker HC, Rensma PL, van Horssen R, de Laat B. Active von Willebrand Factor levels in kidney disease are associated with disease severity and type of dialysis. SSC/ISTH conference, Dublin, Ireland, 2018 (poster presentation).

van der Vorm LN. Salivary tissue factor induces thrombin generation in a diurnal rhythm. 10e Gelre wetenschapssymposium, Apeldoorn, the Netherlands, 2018 (oral presentation).

Van der Vorm LN. Bloedplaatjes-monocyten complexen, endotheel activatie en verhoogde stollingsneiging in patiënten met een acute exacerbatie van COPD. 11e Gelre wetenschapssymposium, Apeldoorn, the Netherlands, 2019 (oral presentation).

Van der Vorm LN, Li L, Huskens D, Hulstein JJJ, Roest M, de Groot PG, ten Cate H, de Laat B, Remijn JA, Simons SO. Acute Exacerbations of COPD Induce a Prothrombotic State through Platelet-Monocyte Complexes, Endothelial Activation and Increased Thrombin Generation. Virtual ISTH conference, 2020 (ePoster presentation).

\section{Awards}

Young Investigator Award. ISTH, Berlin, Germany (2017)

Abstract Achievement Award. ASH, Atlanta, USA (2017)

Award for best presentation. 11e Gelre wetenschapssymposium, Apeldoorn, the Netherlands (2019) 


$$
\text { Appendix IV }
$$


Dankwoord 



\section{DANIKWOORD}

Het is zover, met het schrijven van dit dankwoord leg ik de laatste hand aan mijn proefschrift. Zoals iedereen van me gewend is heb ik ook dit hoofdstuk niet kort en bondig kunnen houden, maar hier is dat onvermijdelijk: een groot aantal mensen heeft mij de afgelopen 4 jaar direct of indirect geholpen om dit boekje tot stand te brengen, en ik wil hen graag allemaal bedanken.

Als eerste wil ik mijn promotor, prof. dr. Hugo ten Cate, bedanken voor de kans om bij $\mathrm{u}$ te mogen promoveren, voor uw snelle reacties op mijn stukken en al uw hulp bij het afronden van mijn proefschrift.

Mijn eerste copromotor, dr. Bas de Laat. Beste Bas, ik herinner me mijn sollicitatiegesprekken via Skype met jou nog goed; ik was halverwege mijn afstudeerstage in Boston, en ik dacht weinig kans te maken met totaal geen achtergrond in de bloedstolling en geen real-life ontmoeting. Desondanks kreeg ik van jou de kans om bij Synapse te komen promoveren, waarvoor ik je dankbaar ben. Ik wil je bedanken voor je vertrouwen in mijn kunnen en zelfstandigheid, voor de vrijheid die je me hebt gegeven om mijn projecten grotendeels naar eigen inzicht in te richten, en voor de mogelijkheid om meerdere (inter)nationale congressen te bezoeken.

Daarnaast ben ik veel dank verschuldigd aan mijn tweede copromotor, dr. Jasper Remijn. Beste Jasper, vanaf het moment dat ik naar Apeldoorn kwam was jij betrokken bij mijn projecten. Je hebt me geholpen bij het leggen van contacten in het Gelre, het opzetten van nieuwe klinische studies en het vinden van mijn draai in deze heel andere omgeving dan bij Synapse. Dankjewel dat je deur altijd open stond voor het beantwoorden van mijn vragen, het regelen van praktische zaken, het lezen van manuscripten en ethische commissie aanvragen en het delen van onze frustraties over die laatste. Ik wil je ook bedanken dat je me hebt betrokken bij het begeleiden van meerdere studenten en het implantologie project. Ik ben blij dat je me, ondanks je nieuwe baan in Amersfoort, de laatste anderhalf jaar toch bent blijven begeleiden tot het einde van mijn promotie. Tot slot heb je me, nog meer dan ik daarvoor al was, geënthousiasmeerd voor het vak van klinisch chemicus. Bedankt!

Van onschatbare waarde was dr. Dana Huskens. Dana, ondanks dat jij geen officiële plek hebt in mijn promotieteam, vervulde jij voor mij wel de rol van copromotor. Ik kon de afgelopen 4 jaar altijd bij jou terecht, voor werk-gerelateerde vragen, maar ook voor persoonlijke ups en downs en een gezellig praatje. Ondanks dat jij in Maastricht en ik in Apeldoorn werkte heb je me bij het opzetten en uitvoeren van heel veel experimenten 
geholpen: van het maken van meer dan 800 plaatjesfunctietesten in één dag tot het inrichten van mijn eigen mini labjes in het grote lab in Apeldoorn en in een schoonmaak/ opslagkamer in Nijmegen. Ik ben onwijs dankbaar voor al je hulp, en ik heb heel veel bewondering voor jouw efficiëntie, organisatievermogen en oog voor details. Dankzij jouw kritische blik zijn alle manuscripten in dit boekje vele malen beter dan de eerste versies die ik jou stuurde. Dankjewel voor alles!

Leden van de beoordelingscommissie, prof. dr. Heemskerk, dr. Franssen, dr. ir. Henskens, prof. dr. de Maat en prof. dr. Meijers, hartelijk dank voor het zorgvuldig lezen en beoordelen van mijn proefschrift.

De studies beschreven in dit proefschrift zou ik niet hebben kunnen uitvoeren zonder de vele patiënten en vrijwilligers die, vaak meerdere malen, heel wat buisjes bloed hebben afgestaan ten behoeve van mijn experimenten. Heel veel dank daarvoor!

Ik heb het als voorrecht ervaren om tijdens mijn PhD samengewerkt te hebben met veel inspirerende en gepassioneerde collega's in de wetenschap, in Maastricht, Apeldoorn en daarbuiten.

Dear former and current colleagues at Synapse, I'm very grateful to all of you for your help and support. When I was still working in Maastricht but also after I moved to Apeldoorn, you made me feel "part of the team". Cui, Marisa, Erik, Rob and Tessa, we didn't really get to work together on projects but thank you for your help with reagents, it was always nice to talk with you whenever I was in Maastricht. Romy, dankjewel voor onze samenwerking voor de Moli-sani studie. Mark, ik heb het onderwerp van mijn thesis eigenlijk aan jou te danken. Dankjewel voor je interesse in mijn project en je feedback op veel van mijn manuscripten. Adam, thank you for teaching me the art of ellipsometry and of course for your help and input for all plasmin-VWF experiments. Joke, jij staat altijd voor iedereen klaar en blijft volgens mij onder alle omstandigheden de rust zelve. Ontzettend bedankt voor al je hulp bij die lange dagen achter de microscoop voor de flow experimenten, maar ook voor je gezellige praatje, o.a. in de auto naar alle Synapse dagjes uit. Ik durf het nooit meer te hebben over het 'STOP' lichtje op het dashboard.. Iris, naast dat je er altijd was voor een praatje was er niets wat jij niet kon regelen, boeken of bestellen; zonder jouw hulp was het me niet gelukt mijn labje hier in Apeldoorn op te zetten. Veel dank daarvoor. Hilde, wat heb jij een ongekend organisatietalent, bedankt voor al je hulp, met name bij de referentiewaardenstudie, maar ook voor de gezelligheid tijdens congressen in Berlijn en in Dublin. Viola, wij begonnen op dezelfde dag bij Synapse, en hebben in het begin veel samengewerkt om o.a. de TG onder de knie te krijgen. Veel belangrijker dan dat is dat ik mede dankzij jou een supergezellig 
eerste jaar bij Synapse heb gehad: je was mijn vaste roomie tijdens congressen, van de eerste Papendal cursus tot de laatste NVTH. Heel erg bedankt daarvoor en ik wens je alle succes met het afronden van je promotie in Leiden. Suzanne, dankjewel voor je altijd vrolijke aanwezigheid op het lab, de gezellige etentjes en al die keren dat je bloed wilde prikken voor experimenten. Cécile, het was een feestje om met jou samen te werken aan de manuscripten over exercise en hypoxemie; je hebt niet alleen heel veel humor, je bent ook een enorm organisatie- en schrijftalent! Ik ben je heel dankbaar voor de kans om bij te dragen aan de BLO2D studie. Walid, jij was al een maand bij Synapse voordat Viola en ik begonnen. We hebben niet veel samengewerkt, maar ik heb bewondering voor hoe snel je zoveel goede papers hebt gepubliceerd, en natuurlijk voor je presentatie skills op grote congressen. Hopelijk komen we elkaar in de toekomst tegen als KCiOs. Li, thank you so much for all of your help with setting up the PMC assay in Apeldoorn, the CYCLE study in Nijmegen, the measurements for the IMPROVE study and of course for your comments on several manuscripts. Yaqui, thank you for your always cheerful presence at the lab and your help with the platelet function tests and LTA. Jun, Dongmei, Quiting and Shengshi, thanks for the nice talks when I was in Maastricht, I wish you all the best of luck with finishing your PhDs. Prof. dr. de Groot, Flip, dank voor al uw feedback op mijn manuscripten, alle telco's en meetings over (vaak onduidelijke) data en de brainstorm sessies voor nieuwe experimenten, ik heb ontzettend veel gehad aan al uw input.

Beste dr. Simons, beste Sami, wat een ongelofelijk toeval dat jij bij mijn presentatie over de TRICA studie (laten we die maar snel vergeten..) aanwezig was en mij hoorde praten over plaatjesfunctietesten, dat heeft uiteindelijk geleid tot onze gezamenlijke IMPROVE en CYCLE studies. Ik vind het geweldig dat jij als arts echt een hart voor wetenschap hebt. Ook na jouw vertrek naar Maastricht kon ik je altijd mailen, bellen of appen als ik vastliep met de interpretatie van onze resultaten. Eigenlijk hoor je daarom thuis in het rijtje van mijn begeleiders; ondanks dat je niet bij het begin van mijn promotie betrokken was heb je voor mij wel die rol vervuld. Via jou ben ik bovendien in contact ben gekomen met veel andere enthousiaste wetenschappers in Nijmegen, de mensen die direct hieronder worden genoemd, waar verschillende samenwerkingen uit zijn voortgekomen. Ontzettend bedankt voor je enthousiasme, positieve instelling en alle tijd en moeite die je hebt gestoken in onze samenwerking.

Graag wil ik dr. Floor Aleva, dr. Quirijn de Mast en prof. dr. André van der Ven van het Radboud UMC Nijmegen bedanken voor jullie vertrouwen in mij om de data van de PRECOVID studie te analyseren. Ik heb tijdens dit project veel bijgeleerd en weggezakte kennis over de immunologie opnieuw opgehaald. Dank voor de fijne samenwerking! 
Ik wil alle medewerkers van de longfunctieafdeling van Dekkerswald (Radboud UMC) te Nijmegen, en in het bijzonder dr. Hanneke van Helvoort, dr. Jeroen van Hees en Petra Theissen, bedanken voor hun inzet en betrokkenheid bij het onderzoek naar het effect van inspanning en hypoxemie op de stolling bij COPD patiënten.

Dr. Wiek Rensma, dr. Remco van Horssen en Ruben Visser van het Elizabeth TweeSteden Ziekenhuis te Tilburg, bedankt voor jullie vertrouwen en de prettige samenwerking tijdens de studie naar actief VWF bij CKD patiënten.

Beste Joost Brouwers van Implantologie Amersfoort, bedankt voor de fijne samenwerking voor de PIMP studie en het speeksel-trombine generatie project. Heel veel succes met het afronden van je eigen promotie!

In de Gelre Ziekenhuizen Apeldoorn zou ik de neurologen, in het bijzonder dr. Paul Bienfait, en arts-assistenten Neurologie willen bedanken voor hun inzet voor de PLAFIS studie. De studie liep niet zoals we hadden gehoopt, maar het heeft niet gelegen aan jullie motivatie. Daarnaast wil ik de longartsen en arts-assistenten van de afdeling Longziekten bedanken voor hun bijdrage aan de inclusie van patiënten voor de IMPROVE studie.

Dr. van Suylen, bedankt voor de kans om 3 jaar van mijn promotietraject bij het KCHL van het Gelre te mogen uitvoeren en voor het bieden van de "infrastructuur" waarbinnen ik mijn experimenten en klinische studies kon opzetten en vitvoeren. Henk, Stephan en Laurens-Jan, bedankt voor jullie interesse in mijn project. Beste Janine, wat een toeval dat de, in mijn ogen "grondlegger" van de actief VWF assay, nu in het Gelre werkt als klinisch chemicus. Bedankt dat je er na het vertrek van Jasper voor me was voor praktische zaken en vragen over lopende projecten, maar ook over de opleiding tot $\mathrm{KC}$ en gewoon om te kletsen over dingen buiten werk, ook heel belangrijk! Saskia en Muriël, bedankt voor het delen van jullie ervaringen als $\mathrm{KCiO}$, en natuurlijk voor het gezellige praatje. Beste mannen van de automatisering, in het bijzonder Henk, veel dank voor jullie hulp bij het opzetten van de IT/logistiek rondom de TRICA, IMPROVE en PLAFIS studies.

Beste analisten en laboranten van het $\mathrm{KCHL}$ van de Gelre Ziekenhuizen Apeldoorn, jullie zijn met teveel om hier allemaal te noemen, maar ik wil jullie hartelijk bedanken voor jullie hulp bij het bloedprikken op de afdelingen en de prikpoli, jullie hulp op het lab en voor jullie oprechte interesse in hoe het met mijn onderzoek ging. 
Met heel veel plezier heb ik tijdens mijn promotietraject studenten begeleid tijdens hun HBO of universitaire (afstudeer)stages. Alicia, hoewel je project eerst over DOACs zou gaan ben ik stiekem blij dat je uiteindelijk toch moest switchen naar actief VWF. Je bracht altijd een vrolijke noot, zelfs als resultaten tegenvielen. Dankjewel voor jouw bijdrage aan de plasmine-VWF experimenten en aan het chronische nierziekten project, en ik wens je veel succes met je eigen promotieonderzoek in Maastricht. Ceráya, wat heb ik een gezellige tijd met jou gehad in "ons" kantoortje bij de trombosedienst. Naast heel veel kletsen en lachen hebben we ook hard gewerkt aan het PIMP en speekseltrombine generatie project, en dat laatste heeft toch maar mooi tot een publicatie geleid! Ik ben ontzettend blij dat we nog steeds contact hebben, en ik wens je alle geluk toe samen met Erik-Jan. Vera, dank voor jouw inzet voor het (actief) VWF referentiewaarden project. Robbin, ook jij bent van grote waarde geweest voor het implantologie project. Uren heb je door de microscoop naar coupes gekeken en je reisde op en neer tussen Apeldoorn, Amersfoort en Deventer met plaatjesfunctietesten, bloedbuisjes en speekselsamples. Je had zeker geen makkelijk project, maar ondanks de moeilijk te interpreteren uitslagen heb je een mooi verslag geschreven, dankjewel!

Ik wil mijn beste vriendinnen Marloes, Sandra en Jorien bedanken voor hun vriendschap en support. Ondanks dat we alle vier zo verschillend zijn en we ver uit elkaar wonen, voelt het altijd weer direct goed als we elkaar spreken. Jullie waren er met een luisterend oor, een knuffel en afleiding voor me tijdens de moeilijke momenten, en daar ben ik jullie heel erg dankbaar voor. Ik hoop dat we nog lang niet uitgekletst zijn en ik ben heel benieuwd wat de toekomst ons alle vier gaat brengen.

Sevgili Ayşin ve Umran Nargül, Öncelikle beni içtenlikle ailenizin bir parçası olarak gördüğünüz için teşekkür ederim. Türkiyeye gelip, sizi ziyaret etmek beni her zaman mutlu ediyor. Bana karşı hep çok nazik ve sevgi dolusunuz. Sizin gibi bir ailem olduğu için çok mutluyum. Beni her gün bu kadar mutlu eden adamı yetiştirdiğiniz için de sizlere çok teşekkür ederim.

Lieve zus en zusje, lieve Anne en Willemijn, ondanks dat jullie beide interesses in een (toevallig hetzelfde) heel ander werkveld dan de wetenschap liggen, hebben jullie altijd belangstelling getoond in hoe het met mijn projecten ging en kon ik bij jullie mijn hart luchten tijdens alle pieken en dalen de afgelopen 4 jaar. Ik ben super trots op jullie allebei. Remco en Alexander, bedankt voor de gezelligheid tijdens gezamenlijke etentjes en feestdagen in Twello, zorg goed voor mijn zus(je)!

Lieve Papa en Mama. Dat de afgelopen 4 jaar best wel een rollercoaster waren had grotendeels te maken met wat er gebeurde op 11 oktober 2017, toen het onderwerp 
van mijn onderzoek ineens heel dichtbij kwam. Dat ene bloedpropje heeft ontzettend veel veranderd, maar wat niet is veranderd is dat jullie er altijd voor mij waren, mij op alle mogelijke manieren hebben gesteund en daarmee gezorgd hebben dat ik hier nu sta. Ik hou van jullie!

Allerliefste Emir, dear Emir. I can't thank you enough for your unconditional love, support and patience with me. I wouldn't have been able to complete this thesis without you. You were and are always there for me, to celebrate the victories but also to offer a listening ear and, if things get really bad, a shoulder to cry on. I'm so proud of you and I can't wait to call you my husband. I'm looking forward to see what the next chapter in our life will bring us, I love you, seni çok seviyorum. 

Appendix V 
List of abbreviations 



\section{LIST OF ABBREVIATIONS}

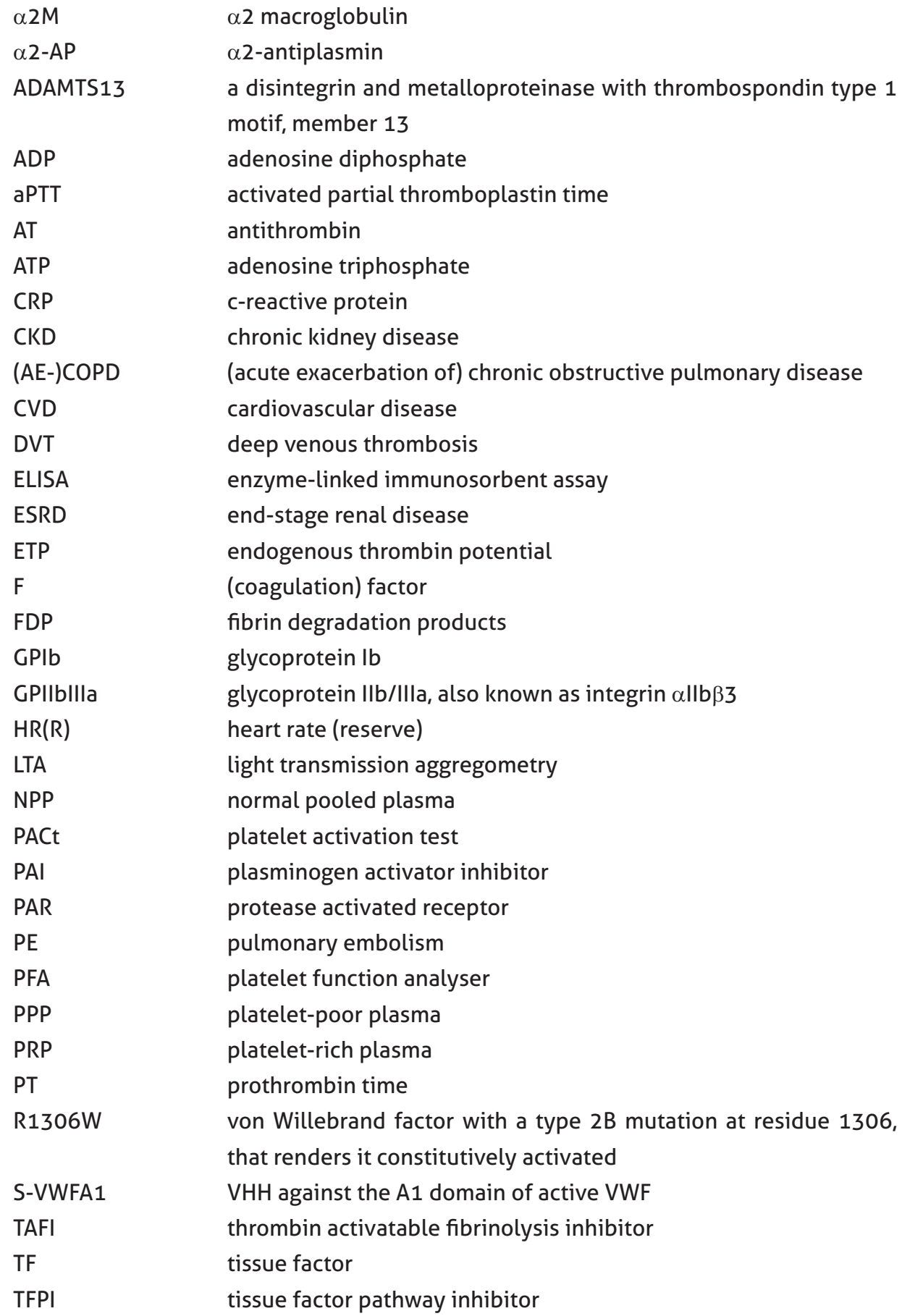




$\begin{array}{ll}\text { TG } & \text { thrombin generation } \\ \text { TM } & \text { thrombomodulin } \\ \text { tPA } & \text { tissue plasminogen activator } \\ \text { TTP } & \text { thrombotic thrombocytopenic purpura } \\ \text { UL-VWF } & \text { ultra-large von Willebrand factor multimers } \\ \text { UPA } & \text { urokinase plasminogen activator } \\ \text { VHH } & \text { variable heavy chain antibody fragment } \\ \text { VI } & \text { velocity index (of thrombin generation) } \\ \text { VTE } & \text { venous thromboembolism } \\ \text { VWD } & \text { von Willebrand's disease } \\ \text { VWF } & \text { von Willebrand factor } \\ \text { VWF:Ag } & \text { von Willebrand factor antigen } \\ \text { VWF:RCo } & \text { von Willebrand factor ristocetine cofactor activity } \\ \text { VWFpp } & \text { von Willebrand factor propeptide }\end{array}$

